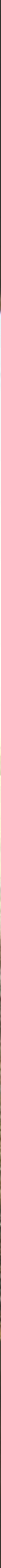




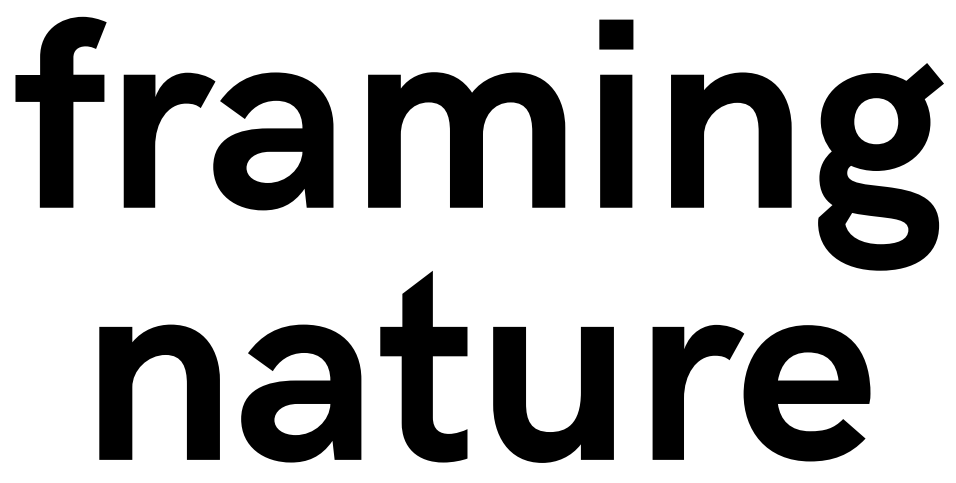

Searching for (implicit)

religious elements

in the communication

about nature

Peter Jansen 


\section{framing nature}

Searching for (implicit) religious elements

in the communication about nature

\section{THESIS COMMITTEE}

\section{Promotor}

Prof. Dr H. Jochemsen

Special Professor Reformational Philosophy

Wageningen University \& Research

\section{Co-promotors}

Prof. Dr F.W.J. Keulartz

Emeritus Professor Environmental Philosophy

Radboud University Nijmegen

Dr J. van der Stoep

Professor Journalism and Communication

Ede Christian University of Applied Sciences

\section{Other members}

Prof. Dr M.N.C. Aarts, Wageningen University \& Research

Dr M.A.M. Drenthen, Radboud University Nijmegen

Dr A.C.M. Roothaan, VU Amsterdam

Prof. Dr M.G.C. Schouten, Wageningen University \& Research
Peter Jansen

THESIS

submitted in fulfilment of the requirements for the degree of doctor at Wageningen University

by the authority of the Rector Magnificus, Prof. Dr A.P.J. Mol,

in the presence of the

Thesis Committee appointed by the Academic Board

to be defended in public

on Wednesday 14 June 2017

at 4 p.m. in the Aula.

This research was conducted under the auspices of the Wageningen School of

Social Sciences 


\section{Table of Contents}

Peter Jansen

framing nature

Searching for (implicit) religious elements in the communication about nature 200 pages

PhD thesis Wageningen University, Wageningen, the Netherlands (2017)

With references, with summaries in English and Dutch

ISBN -978-94-6343-132-3

DOI $\quad 10.18174 / 409678$

\section{GENERAL INTRODUCTION}

Introduction

Government communication and changing society

Nature experiences and religious subtexts

Casestudy Tiengemeten

Outline of the thesis

\section{THEORIZING GOVERNMENT COMMUNICATION}

WITH REGARD TO THE DUTCH NATURE POLICY

Contextual turn

Closer examination of communication

Discussion

11

15

17

19

20

GOVERNMENT COMMUNICATION AS A NORMATIVE PRACTICE 49

Introduction

Normative structure of practices

Structural analysis of government communication

Discussion

IN SEARCH OF RELIGIOUS ELEMENTS

IN THE DUTCH NATURE POLICY

73

Introduction

Socio-historical context of the Dutch nature policy

Theorizing views of nature

4.4 Discussion 95 


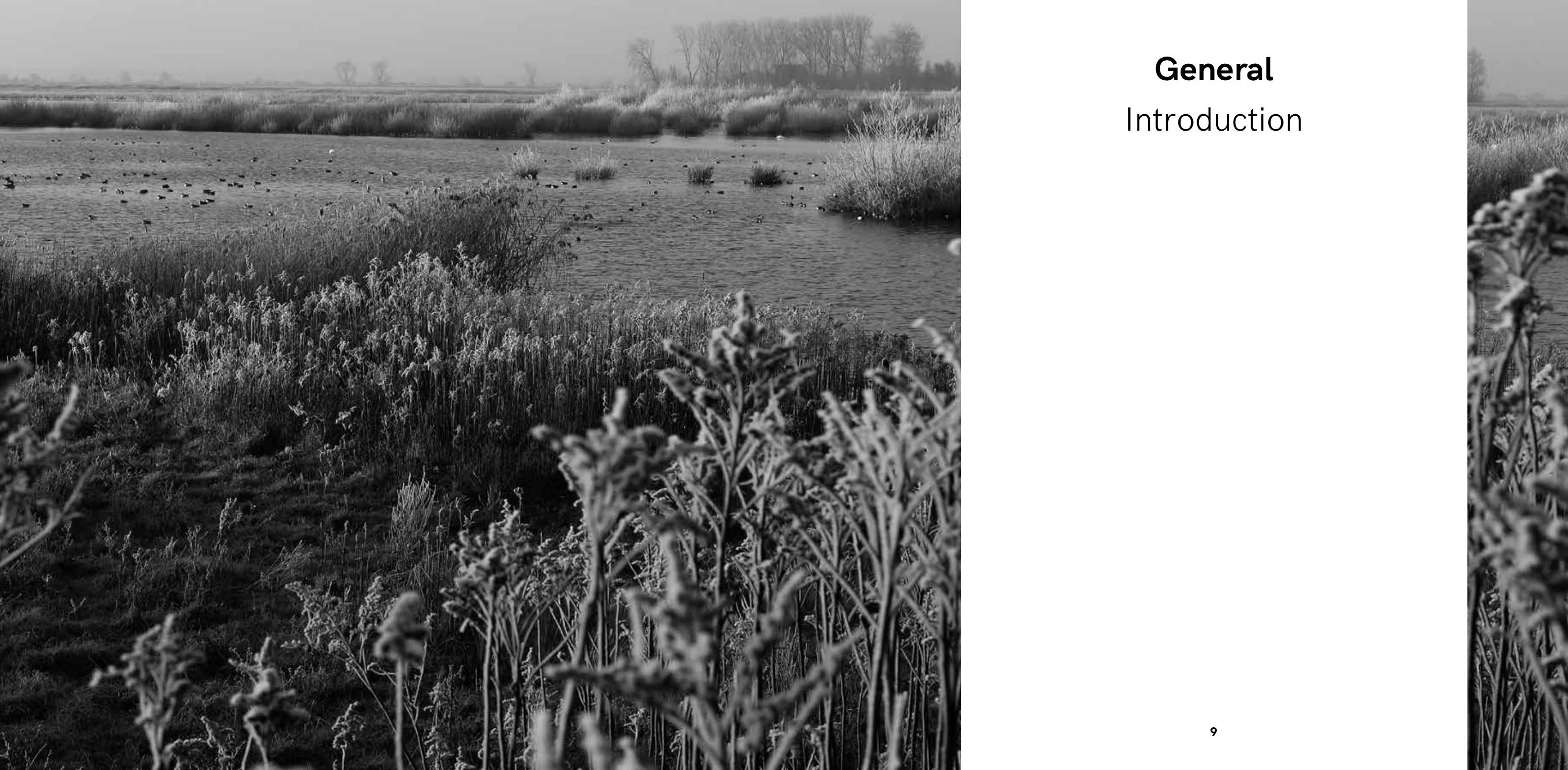




\section{General \\ Introduction}

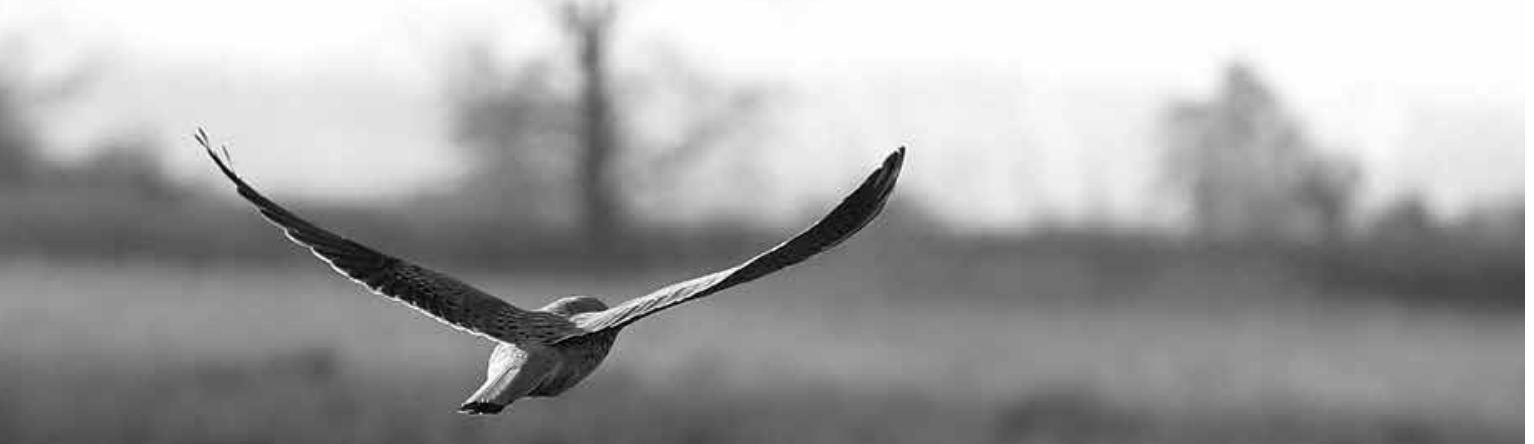

Tiengemeten (...)

It falls outside our order.

And that changes everything.

The traditional residents of Tiengemeten were

farmers, families who had lived there since time immemorial

and who knew everything about

timelessness and patience.

Then the creators came alongside God, the engineers of meadows, marshes, flowers and birds, enforcers of order in its most extreme form,

the tamers and divers of Circus Nature.'

$$
\sim \text { GEERT MAK }{ }^{1}
$$

1.1

\section{INTRODUCTION}

For more than one thousand years, the Dutch have wrested land from the sea. The Dutch transformed their country from a swampy river delta into an ordered land with a significant agricultural image. 'Cattle instead of sheep have been Dutch rural icons since van Ruysdael's 17th century paintings,' Lowenthal (2007) argues, 'a dappled hide against a green landscape is more a logo of the Netherlands than the tulip' (646). However, on 7 March 1993, The New York Times published an article with the intriguing title: 'Dutch Do the Unthinkable: Sea Is Let In'. The journalist notes with some surprise that the Dutch intend to make holes in dikes and flood their fertile country (again). 1 Quote taken from the preface to the book Over het Vuile Gat (2007) by Ton van der Graaf.
This 'flooding of the country' is a result of a more offensive approach by the Dutch government that 
was launched as part of the Nature Policy Plan presented in 1990 (Zwannikken, 2001). The period up to 1990 was characterized by increasing attention for nature conservation and the adoption of a so-called defensive policy. Attempts were made to retain what existed but, at the same time, it became clear that terrain was being consumed, and nature was losing out (Kockelkoren 2000). In other words, a more offensive approach was deemed necessary as the number of plants and animals was shrinking, biodiversity was decreasing, and the land devoted to nature in the Netherlands was dwindling (Hajer, 2003). An important consequence of this more offensive approach is the achievement of a National Nature Network, known as the Ecologische Hoofdstructuur before 2013 and generally abbreviated as EHS. Within this national nature network, the conservation, restoration, and development of nationally and internationally important ecosystems are given shape. As a consequence, in recent decades, the Dutch government has purchased a substantial amount of land to give 'back to nature'. In a sense, this is a second transformation, i.e., from agriculture into nature. Communication about this second transformation is my topic of interest and is central to this $\mathrm{PhD}$ thesis. This study concentrates on government communication. However, it appears that NGOs are intimately involved in the realization of the new nature policy and the communication about it. This situation requires clarification.

In the so called Natuurakkoord, established at the end of 2011, it was agreed that the central government of the Netherlands is responsible for the framework (laws and regulations) and ambitions of nature policy. Based on the Wet Natuurbescherming, effective since the beginning of 2017, the central government is obliged to compose a national nature vision whereby the government broadly describes the Dutch nature policy. However, the provincial governments are responsible for the completion, i.e., concretising and implementing the nature policy in the Netherlands. This is mostly accomplished in collaboration with nature conservation NGOs such as Natuurmonumenten, Staatsbosbeheer, and the various Provinciale Landschappen. The involvement of nature conservation NGOs is, according to Van der Zouwen (2006), a traditional characteristic of the Dutch nature policy. Hence, she speaks about the intermingling of governmental and nongovernmental actors. With regards to the Veluwe, a well-known forest-rich nature reserve in the Netherlands, she concluded that, although the national government brought in budget and designated the area as an important core area with regards to the realization of the EHS, the national government has no dominant position because of the dependency of what she refers to as 'diverse landowning actors' (199). 'Those who own land have the ability to clearly leave their mark on nature policy,' (200) she argues.
In this $\mathrm{PhD}$ thesis, I will argue that the government is primarily juridically qualified (cf. Zwart, 1994), and nature conservation NGOs are value-oriented and, therefore, rather morally qualified. However, in the collaboration between the government and the NGOs, the government supports with its instruments (laws and regulations, including budget) the (moral) value orientation of the NGOs, i.e., the realization of the values of the NGOs. At the same time, the NGOs execute the nature policy of the government. A good example of such a synergistic relationship involving an intermingling of governmental and nongovernmental actors is the following quotation from page 5 of the vision document 2010 - 2022 of Tiengemeten:

Natuurmonumenten wants to develop the concept of nature island

Tiengemeten together with the provincial and local government and with the several operators on the island: a guided natural freshwater tidal landscape, with distinctive culturalhistorical opportunities for nature and landscape experiences, where the visitor experiences space and quiet.

Natuurmonumenten is dependent on support from governments, business companies and individuals to durable maintain nature island Tiengemeten.

Similar to, for instance, the Dutch private broadcasting organisations with the money they receive from the government, nature conservation NGOs function as delegated executors of the Dutch policy. Otherwise stated, established Dutch nature policy is de facto executed through nature conservation NGOs, leaving them a degree of freedom within the contours of government policy. As a consequence, although the focus of this study is on communication that is actually produced by an NGO (chapters $4-6$ ), the synergistic relationship of this NGO and the government, at least in the example of Tiengemeten, implies that the communication can actually be considered as (also) government communication on that iconic example of the new nature policy of the Dutch government. This, in my opinion, justifies that we analyse that communication as government communication (chapters 2 and 3) and as governmental nature policy (chapter 4).

As mentioned above, the focus of this $\mathrm{PhD}$ thesis is on communication that is actually produced by NGOs. In the communication of NGOs, a sharp distinction is made between 'real' nature and landscapes that are influenced by man. Metaphorical words and images are used, e.g., 'where you hear the silence, feel the emptiness' or, as illustrated in the quotation below:

There is something grand and wonderful on the island Tiengemeten. The whole island, one thousand acres, is just pure nature. True wilderness, with 
flowing creeks and gullies where you can walk endlessly through paved and trackless terrain, while the mighty sea eagle circles over your head. On the way you rest a while at the eastern tip of the island, where the atmosphere of the countryside of 1850 comes back to life (flyer DeltaNatuur, 2005).

At times, it appears that the words that are used appeal to a search for meaning as well contain in a sense 'religious subtexts' (cf. \$1.3). For example, in a booklet about De Oostvaardersplassen, we can read that this nature reserve tends to 'enrich people's vision and spirit', the park 'enables us to powerfully experience the primal force of nature and to learn from it'. On the wall in a visitors center in this park is written: "nature goes its own way as much as possible, it follows the rhythm of the seasons, the circle of life and death (...) they are, here and now, no more and no less, where heaven and earth meet'. In a magazine of Natuurmonumenten, I read the sentence that 'when you step off the boat in Tiengemeten, you enter a new world'. When you arrive on this island, the following quote can be seen: 'deliberate act of creation'. And finally, a magazine about nature in the Netherlands

states the following: 'almost every nature-lover can remember as clear as day a moment when they became one with their surroundings: call it magical, call it divine'. There are just a small number of examples, however, they raise the question: what is the background for the use of this type of 'heavily value-laden language' in the Dutch discourse about nature? ${ }^{2}$ Is it just an expression of a clever marketing strategy that aims to 'sell new nature', or can we identify here a symptom of a change in the experience of nature in Dutch society? Against this background in my $\mathrm{PhD}$ thesis, I examine the communication about nature in the Netherlands, in particular searching for (implicit) religious elements in this communication. My aim is to gain insight into the role of these (implicit) religious elements in the communication. By doing so, I hope to better understand the Dutch discourse about nature. Hence, this study will provide an answer to the following main research question of whether and, if so, which (implicit) religious elements play a role in the communication about nature (policy) in the Netherlands?

\begin{abstract}
2 In this respect, the tripartite ap proach of Frith (1998) for analysing ads is interesting: (i) 'the surface meaning', (ii) 'the advertiser's intended meaning', and (iii) 'the cultural or ideological meaning'. With respect to the surface meaning, she argued that it 'consists of the overall impression that a reader might get from quickly studying the ad vertisement. You can describe this surface level of meaning by simply listing all the objects and people in the ad' (5). She continues: 'the Advertiser's Intended Meaning is the sales message that the advertise is trying to get across. Some mar keters refer to this as the strateg behind the ad. It is the 'preferred' or 'expected meaning' that a reader might get from the ad; the meaning that the advertiser intends for the reader to take with them' and 'the Cultural or Ideological Meaning relies on the cultural knowledge and background of the reader. We all 'make sense' of ads by relating them to our culture and to the shared belief systems held in com-
\end{abstract}

The subject matter of the national nature network is complex as it is not just a spatial-ecological task. As Hajer (2003) stated: 'environmental policy should not be regarded as a fixed programme for ecological improvement that 'only' needs to be implemented' (92). ${ }^{3}$ Various parties with wide-ranging interests, ideologies, and visions are involved in the achievement of a national nature network. In the terminology used by Van der Ziel (2003): 'there are 'power plays' hiding behind landscapes, an arena of interests, a battlefield of representations' (6). Despite the fact that consultation practices during the process of policy making and implementation include a broad range of organisations, Hajer (2003) concludes that local protests show that (nature) organisations are currently not reliable representations of feelings at the local level. This indicates that a new perception of the relationship between government and society has emerged in the Netherlands since the 1990s (cf. Keulartz et al., 2004). Citizens are not just nationals and voters but are increasingly also negotiators, policy co-creators, and clients/customers of this policy (Middel, 2002; Jumelet \& Wassenaar, 2003). Otherwise stated, citizens are increasingly not objects (of communication) but co-subjects and actors. In my second chapter, this contextual turn, as I call it, will be examined and theoriszed in more detail.

However, the question is urgent to what extent the observed contextual turn affects government communication. Hence, a more comprehensive review of government communication is necessary and will be provided in the first of the following chapters. Also, the role of the government communication practitioner must be explored. In 1991, the Court of Audit stated that there should be less of an emphasis in government communication on influencing behavior (Olsthoorn \& Van der Velden, 2007). However, Pol (2008) refers to this as 'the fatal blow' as a result of which 'communication professionals ran in all directions' and 'in doing so lost sight of the main activities. Namely: influence' $(7+8)$. It is precisely this influence that, according to him, is the old trade of government communication. The government's influence on behavior is regarded by Pol et al. (2007) '[as] part of its task and necessary to be able to provide a solution to problems in society' (15). According to them, communication that influences behavior is 'the greatest challenge for the government or, rather, 'it ought to be' (20). Therefore, there is a

3 In the Dutch discourse about nature, it is more common to refer to 'nature policy' rather than 'environmental policy'. lack of clarity about the role of government commument communication from a perspective that considers this as a 'practice' is included in the third chapter. 
MacIntyre (1984) defines a practice as a socially established and complex activity designed to cooperate with each other in order to achieve the objective or destination of the practice. The words 'objective' and 'destination' are important in this context. This means that the activities of a practice are not characterized primarily by technical skills but instead aim to achieve the 'objective or destination' of the practice. I follow Jochemsen (2006) who, on the basis of research conducted with other people, provides an elaboration of the practice concept of MacIntyre with the aid of Dooyeweerdian, i.e., reformational philosophy (see Textbox 1). In the so-called 'model of normative practices', Jochemsen (2006) maintains the concept of the 'telos' of a practice. He argues that 'the 'telos' of a practice belongs to the very nature of the practice and is not founded in the intention of the practitioner' (103). However, the question is what can be defined as the 'telos' of government communication. In my third chapter, in which I seek an answer to the question as to whether the model of normative practice is beneficial for clarifying the task of government communication practitioners, I elaborate on this question in more detail.

\section{TEXTBOX 1. Reformational Philosophy}

The model of normative practices (NPM) is designed to better understand the character of a certain professions. 'NPM is in essence a theory on professions, which can help the reflection on what is at stake in a profession' (Jochemsen et al., 2006:9). The background of the NPM can be found in reformational philosophy. ${ }^{4}$ This philosophy is based on the work of the Dutch scholars Herman Dooyeweerd (1984 - 1977) and Dirk Th. Vollenhoven (1892 - 1978), both part of the neo-Calvinist tradition. According to Glas (2012), three ideas are fundamental for reformational philosophy:

1. Everything that exists functions in a plurality of modal aspects (or ways of being; ways of functioning), hence, reformational philosophy

is fundamentally anti-reductionist.

2. None of these modal aspects can be reduced to other modes.
4 Van Woudenberg (2009) rightly notes several names have appeared over the last few decades, e.g. 'Calvinistic philosophy' and 'Wijsbereerte ly translated as 'the Philosophy of the Law-Idea', after the English title terpiece in 1935/1936, or as 'Phlosophy of the Cosmonomic Idea'. It is noteworthy that Dooyeweerd employs the phrase 'Wijsbegeert der Wetsidee' in the title of his oration as professor at the Vrije Universiteit Amsterdam in 1926. In the last decennia, especially in the Netherlands, the name 'Christian philosophy' is used. Currently, the Association for Reformationa Philosophy, established in 1935 with aid of Dooyeweerd and Vollenhoven has six special chairs in 'Christia (Reformational) philosophy' at sev eral universities in the Netherlands: Delft, Eindhoven, Leiden, Maastricht, Twente, and Wageningen. of Herman Dooyeweerd's first mas -
3. Nevertheless, there exists a strong coherence between the modal aspects.

An important distinction within reformation philosophy that is noteworthy is the distinction between structure and direction. There is a structural and a directional side to the way things develop over time. The structural aspect refers to the modal aspects (ways of functioning) in which a thing functions and the order and coherence between these aspects. The directional aspect refers to the fact that structures can be opened up in a process of disclosure in time that will be guided by the fundamental beliefs of those who live the structure, for example, a department of government (cf. Glas, 2012).

The founder of reformational philosophy, Dooyeweerd, contended that 'when parts of reality are opened up in a wrong (non-normative) direction, this will inevitably lead to antinomies (i.e., to tensions that will harm the development of that thing (process, larger whole)' (Glas, 2012:4). This insight will turn out to be relevant when discussing the communication about

\subsection{NATURE EXPERIENCES AND RELIGIOUS SUBTEXTS}

In the fourth chapter, I present a socio-historical overview of the Dutch nature policy (cf. De Jong, 1999). I discuss the particular way nature has been constructed and managed in the Netherlands. In the discourse about nature, there are all sorts of ideas and concepts about nature doing the rounds. In the literature, the term representations, i.e., mental images of collective ideas of nature, is some-

times used (cf. Van der Ziel, 2003; Buijs, 2009). More common terms are 'images 5 De Groot et al. (2003) refer to of nature' or 'views of nature' (cf. FootNote 64), in 'visions of nature' as an umbrella Dutch known as natuurbeelden. ${ }^{5}$ Keulartz et al. (2004) term with two elements: (i) 'images describe natuurbeelden as being socially-culturally of nature', defined as types of na- determined views regarding the character, the value, 'images of relationship', defined and the appreciation of nature. ${ }^{6}$ The final result is that as images that people have of an the natuurbeelden show how people think about nature appropriate relationship between people and nature. This is in line and what they think about how we should treat it. with Buijs (2009), who states that In a certain sense, the natuurbeelden can be regarded natuurbeelden have two dimensions: (i) somebody's definition of nature and (ii) their normative notions of nature. 2004). To state it another way: natuurbeelden can be regarded as reference frameworks on the basis of the government's nature policy (chapter 7). 
which our nature preferences are shaped and as interpretation frameworks on the basis of which our experiences in nature are given meaning (Keulartz et al., 2002; Buijs et al., 2009).

Van der Ziel (2003) notes that 'reality and the perception and imagination of this reality cannot be separated' (12). He thus states that there is a close relationship between how we experience reality and how we imagine it. In regard to this $\mathrm{PhD}$ thesis, people have experiences with nature that affect how they talk about / imagine nature. It is interesting that research by Van den Born \& De Groot (2011) shows that larger-scale, wild nature is more inviting in regard to unique experiences whereby, consequently, it is easier to be thrilled and to feel a moment of connectedness. As a result of this, several authors regard nature as a site of religious experiences (Van Saane, 2002; Champ, 2009; Ross-Bryant, 2013). Otherwise stated, nature provides an opportunity for meaningful experiences in which a religious depth dimension can be discovered in nature experiences. However, what exactly is this religious depth dimension, and does this mean that any communication about nature contains, in a sense, 'religious subtexts'? In my fourth chapter, a theoretical elaboration will be provided of these types of questions.

In this $\mathrm{PhD}$ thesis, religion has the connotation of implicit religion in which religiosity is regarded as an anthropological structure rather than interpreted in terms of institutions, doctrines, and structures (cf. $\$$ 4.3.3). Otherwise stated, religion has do with that which connects and binds people to what they value most and consider or experience as sacred (Taylor, 2010; cf. FootNote 79 and 103) or, in terms of Clouser (1995), that which (groups of) humans see as 'unconditionally independent reality' (23). It is this concept of and relation to 'the sacred' that provides the content of the directional side of (social) structures (cf. Textbox 1).

This $\mathrm{PhD}$ thesis attempts to 'find religion in expressions of meaning and value that weave through a culture' (Ross-Bryant, 2013:4). I, therefore, deliberately use the term religious elements (cf. FootNotes 80 and 117). The interesting questions now are what exactly are these religious elements which 'weave through a culture' and, more precisely, how do they resonate in the discourse about nature? Whereas the emphasis in chapter 4 is mainly on theory, this topic is detailed in chapters 5 and 6 of my $\mathrm{PhD}$ thesis by using a case study regarding Tiengemeten.
6 Jacobs (2002) refers to the true, the right and the genuine landscape. The true landscape is nature as we know it in objectiv reality and that can be described as such; the right landscape refers to a set of standards, laws, etc. the aim of which is to control behavior with regard to nature; and finally the genuine landscape is nature in which experiences are had to which people attribute personal meaning.

\subsection{CASE STUDY TIENGEMETEN}

Verschuren \& Doorewaard (2000, cf. Plooij, 2011) define a case study as 'a study in which the researcher tries to obtain an in-depth insight into one or more spatio-temporally limited objects or processes' (169). ${ }^{7}$ In his definition, Robson (2002) stresses even more the spatio-temporal aspect when he notes that a case study is a research method that uses empirical material 'of a certain contemporary phenomenon within a current context' (178). In the case of this thesis, this is the religious elements (contemporary phenomenon) in the communication about Tiengemeten (current context). Tiengemeten was selected because the island illustrates the changing way that we think about nature in the Netherlands as the following quote by Metz (1998) illustrates

The changes on Tiengemeten indicate the changing attitude of nature conservation: no longer simply defensively conserving nature that already exists, but taking the offensive and buying land to create nature (Metz, 1998:141)

As the issue of religious elements, as we indicated above, is closely associated with how people experience nature, the experiences of visitors to the island itself and communication about Tiengemeten are central to chapter 5 and 6, respectively.

I opted for semi-structured interviews as a research tool. These offer the possibility of obtaining in-depth information specific to each individual, such as experiences in nature. In chapters 5 and 6, I elaborate the methodology of the case study in greater detail, and I will reflect on my selected research method in the general discussion (chapter 7). At the moment, it is important to note that semi-structured interviews are in accordance with a hermeneutic approach to research whereby the researcher operates as 'a facilitator and translator of the shared meaning that is generated (...). The story that is forefront in the participant's memory is the story the interviewer facilitates' (Vandermause \& Fleming, 2011:371+373). Researcher and respondent 'work together to generate an understanding as narrative text emerges and language is interpreted' (Vandermause \& Fleming, 2011:371). In a certain sense, a hermeneutic research approach is an interaction

7 Various authors (Verhoeven, between obtaining factual knowledge and extending 2007; Swanborn, 2008; Plooij, the knowledge gained from experiences. It is about 2011; Baarda et al., 2012), there- attempting to understand reality 'from the point of fore, also refer to a case study as $\quad$ view of the person who experiences it' according to axt De Boer \& Evers (2007:8). They then note that what 
the respondent says during the interview is maybe not 'the truth' in a factual sense 'but it is a recordable statement that can be interpreted and placed alongside other statements' (9). In the terminology used by Gadamer ([1975], 2004), my PhD research is about 'outside science' which, in this context, means that my aim is not to consider individual experiences as statements of general rules or confirmations and refutations of general laws (cf. Oudemans, 1988). In this sense, my goal is not a generalisation of research data but an attempt to identify links that contribute to a better understanding of natuurbeelden and the role that religious elements play in the communication about nature.

\subsection{OUTLINE OF THE THESIS}

As mentioned at the beginning of this chapter, in this $\mathrm{PhD}$ thesis I examine the communication about nature in the Netherlands, in particular searching for (implicit) religious elements in this communication. To summarize and as a reading guide, based on a literature study in the second chapter, I examine the question of the extent to which the new perception of the relationship between government and other parties influences the discourse about the nature policy in the Netherlands. Therefore, a comprehensive review of communication is necessary. As a consequence, in my second chapter, I also briefly address the question: what is government communication? The third chapter aims to further clarify the structure of government communication practice. An answer is sought to the question of whether the model of normative practice, as developed within the reformational philosophy, is beneficial for clarifying the task of government communication practitioners. The fourth chapter begins with a socio-historical overview of the Dutch nature policy. Based on a literature study, I discuss the particular way nature has been constructed and managed in the Netherlands, and I subsequently conceptualise the manifest views of nature in Dutch nature policy. The fifth chapter builds on this primarily conceptual fourth chapter. Based on qualitative research, it begins with the question of whether the discourse of key actors corresponds to visitors' descriptions of their experiences of Tiengemeten. In order to answer this question, the discourse of people who were either involved from a policy perspective in the transformation of Tiengemeten or who played an appropriate and influential role in the public communication, socalled key actors, is examined. Subsequently, I investigated how visitors evaluate the discourse about Tiengemeten in regard to their own experience of nature on the island. The final section of this fifth chapter both compares and discusses the implications of the findings for (communication) policy and strategy. The aim of the sixth chapter is to use the case study about Tiengemeten to gain greater insight into the meaning of nature experiences and views of nature in the life of nature conservancy area visitors. Hence, the central question of this explorative chapter is what layers of depth can be identified in the description of the experiences of visitors to Tiengemeten? The last two chapters collectively provide qualitative data for answering the question of what the religious elements are and how they resonate in the discourse about nature. In the seventh chapter, I want to engage in a general discussion of the main results of my research in light of other literature. 


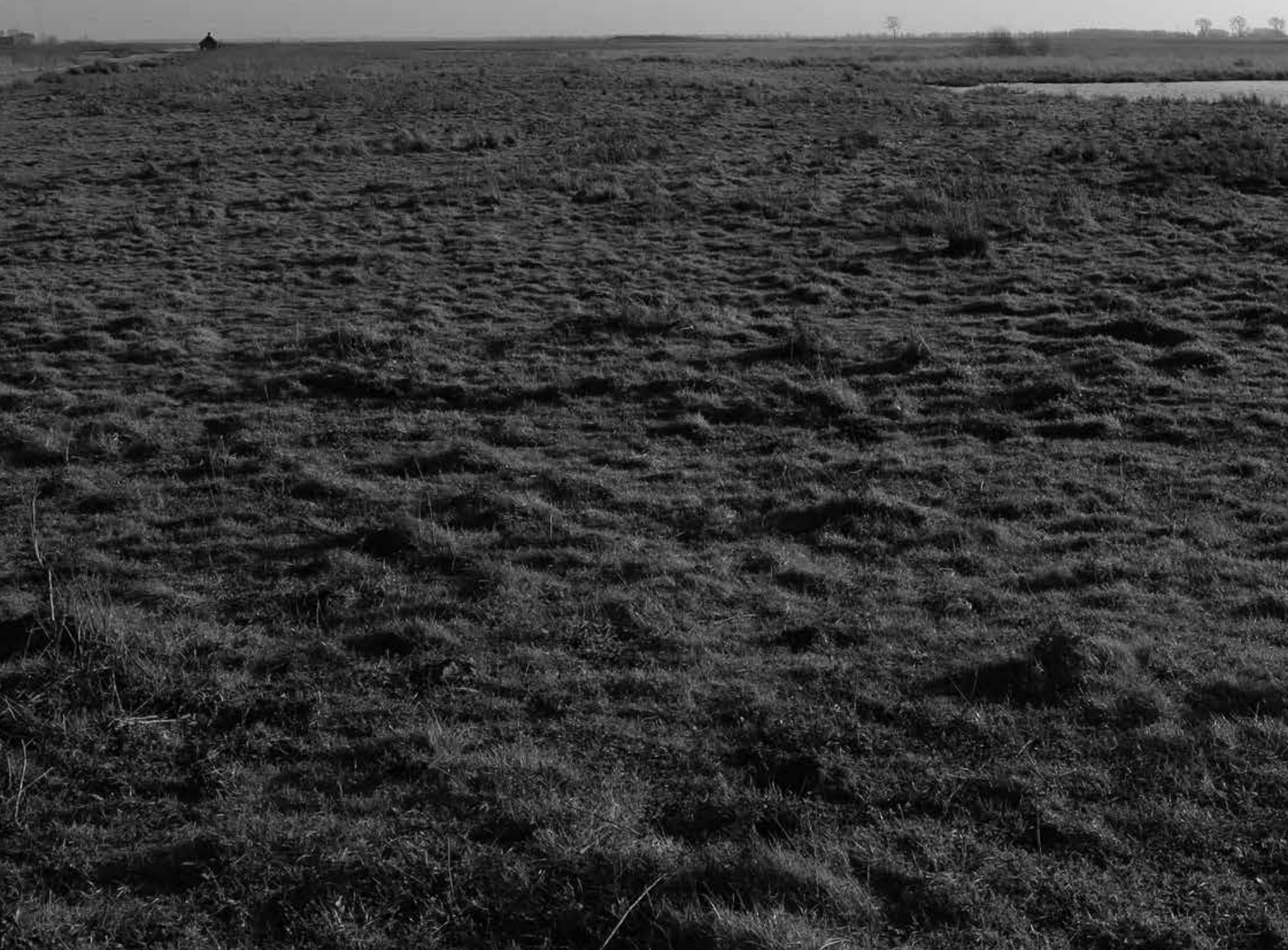

\section{Theorizing Government \\ Communication \\ with regard to the Dutch \\ Nature Policy \\ A version of this chapter is accepted fo publication as P. Jansen, J. van der Stoep $\& \mathrm{H}$. Jochemsen (in press). Theorizing Government Communication with \\ regard to the Dutch Nature Policy. Empe- docles: European Journal for the Philosophy of Communication; it will be published in issue 8 (1), June 2017.}




\section{Theorizing Government}

Communication with regard to the Dutch Nature Policy

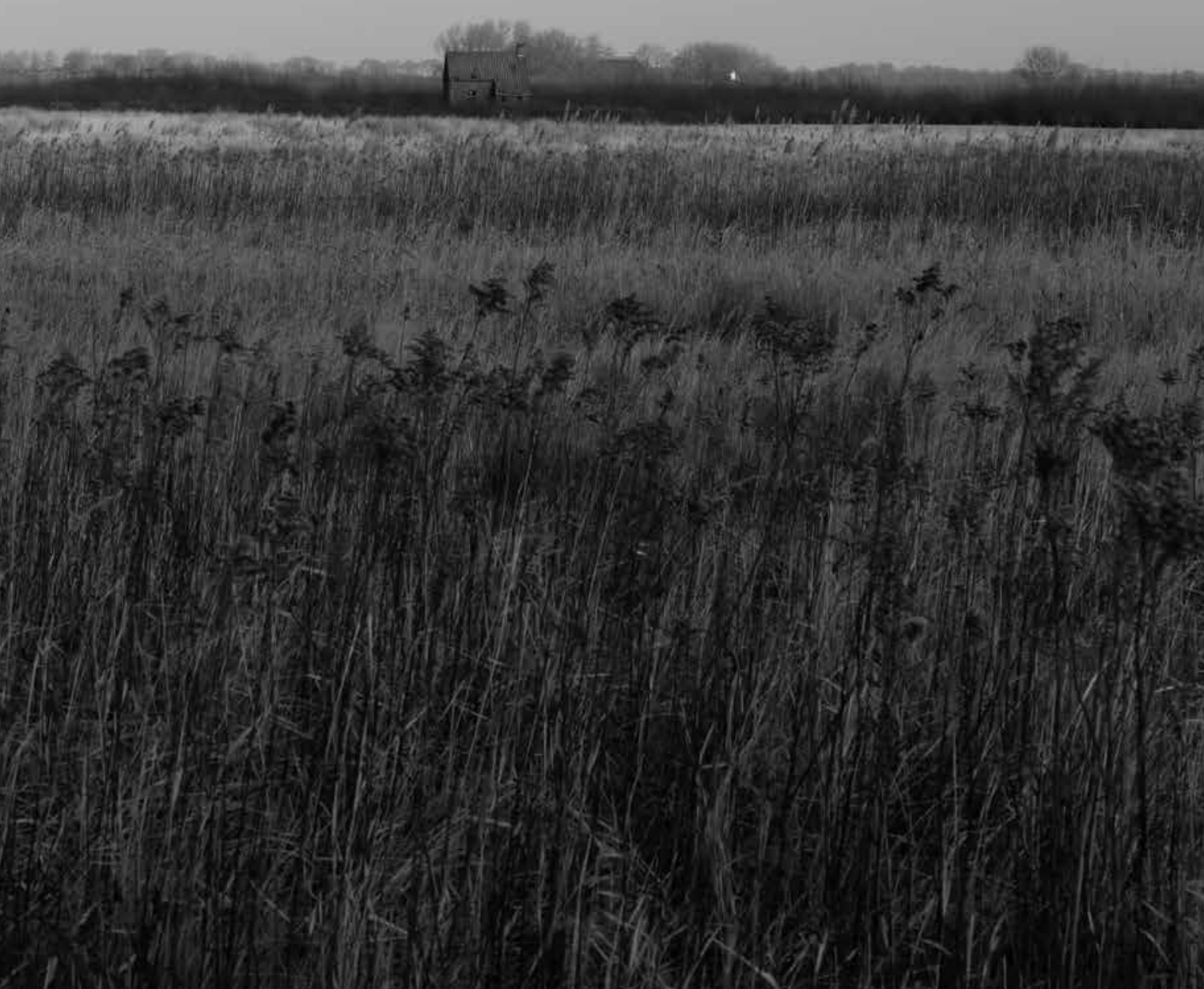

ABSTRACT - The implementation of a National Ecological Network poses a significant challenge to the Dutch government. The establishment of this ecological network has led to conflicts among various interest groups in the public sphere, each of which defends its own interests. In this struggle for recognition communication fulfils an important role. This chapter contends that the discourse about nature is driven by deep frames, is comprised of values and is rooted in worldviews. The insight that worldviews play a role elucidates the various positions in the debate and shows normative dimensions in communication. This paper argues that the network society, more than ever, requires the government to be explicit about its normative choices.

\section{INTRODUCTION}

Today we find ourselves living in a network society. Castells (2009) writes that in a network society each participant is a sender who can determine who receives his or her messages. At the same time each participant is a receiver who can determine from which sender he or she would like to receive messages. As compared to the industrial society that preceded it, this means a shift from allocution - where the sender determines what, when, and via which medium messages are communicated - to consultation - in which the sender makes information available and the receiver determines what, when, and by which medium that information should be accessed. ${ }^{8}$ This development has

of society, prevailed (cf. Fischer, consequences for the relationship between government and interest groups or non-government organizations (NGOs). Since the 1990s a new perception of the relationship between government and society 
control' to more 'contract and negotiation' (Keulartz et al., 2004). The government thus increasingly becomes a participant and actual policy becomes a product of consultation and bartering. In this chapter we want to gain an insight the extent to which this new perception of the relationship between government and other parties influences the discourse about nature policy in the Netherlands. ${ }^{9}$

The implementation a National Ecological Network [NEN] has for many years been the most prominent ideal for nature policy in the Netherlands (cf. $\int$ 1.1). A network of nature conservation areas is being created throughout the Netherlands to help preserve the country's flora and fauna. The typical Dutch landscape is disappearing and, in order to preserve Dutch nature 'in which many varieties of flora and fauna can live' (Ministerie van LNV, 2005: 3), the government needs to supervise the restoration of nature in certain areas. Hence, Dutch nature policy can be considered to have a pro-active strategy for conservation; its primary goal is not to protect, but rather to extend nature areas (Swart et al., 2001). However, achievement of the NEN in practice is difficult: it is not citizens and politicians who determine the direction of nature policy, but rather scientific experts, Keulartz (2009) suggests. In a sense there is a discrepancy between the science-based (ecological) knowledge upon which nature policy has heavily relied and local, experience-based knowledge, i.e. local perceptions and views (Swart et al., 2001; Keulartz, 2005). In this respect that which Hajer (2003) argues is intriguing: political conflicts are related not merely to the intended change of policy 'but at least as much to the institutional way of conducting politics' (89). In his view nature development in the Netherlands illustrates 'the limited effectiveness of classical-modernist political practices in dealing with these kinds of complexities and with the fragmented and unanticipated political dynamics these generate' (89).

As mentioned above in this chapter, we examine to what extent new perceptions of the relationship between the government and other parties influence the discourse about nature policy in the Netherlands. In the first part of this chapter we argue that the character of government communication has changed. We have called this the contextual turn. In the second part of this chapter we explore the issue of normative dimensions in communication, in relation to frames that exist in language. Therefore a closer review of communication is necessary. Central to this chapter is the thought that without both an awareness of the contextual turn and insight into the normative dimensions in communication the discourse about the Dutch nature police cannot be properly understood.
9 In this chapter we adopt Hajer's (1995) description of discourse. He defines discourse as an ensemble of ideas and concepts that ar 'produced, reproduced and transformed in a particular set of practices' (44).
Drawing on our theoretical research we argue that the network context, more than ever, requires the government to be explicit about its normative choices. This leads us to briefly deal with the concept of argumentative turn.

\subsection{CONTEXTUAL TURN}

As already mentioned, a different relationship between the government and other social actors has developed in the Netherlands. According to authors such as Keulartz (2005), this perception concerns a shift that took place towards socialisation and commercialisation within the public sphere. Keulartz (2005) claims that this move towards socialisation and commercialisation entails a double shift. There is, firstly, a perceived upward and vertical shift of public accountability from the national to the supra-national level. To a large extent the issues of the nature policy transcend the regional scale and scope and therefore require action at a higher level. Secondly, there is a perceivably clear downward and vertical shift from the national level to more regional levels (Keulartz et al., 2004). Here we see the paradox of globalisation: globalisation is accompanied by increasing regionalisation, which causes the national level to recede from view. ${ }^{10}$

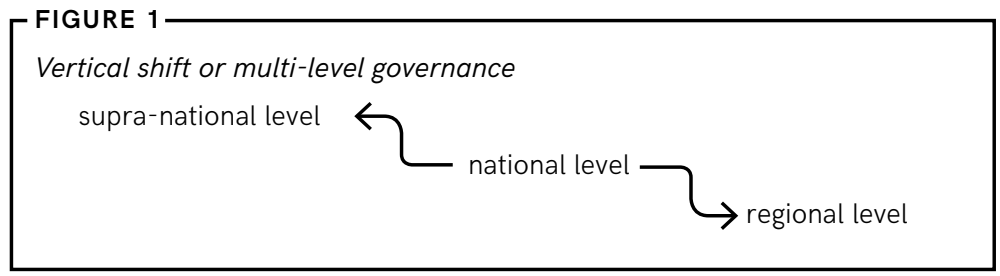

This double shift demands not only multi-level governance (see figure 1) but also

10 Bardoel (2000) talks of the paradox of modernity and claims that modernisation is straightforward but 'a paradoxal process that generates opposing trends' (32). He writes in this respect: 'We perceivel the rise of a global culture, which commenced with internationalization (...) some thirty years ago. (...) A segment of this process of globalization is the postulated erosion of the nation-state, which after all, is 'too small for large problems and too large for small problems'. As a result, our continent knows two parallel processes. On the one hand, there is the process of European unification and indeed of advancing globalization, while, on the other, we see, as the counterpoint, a renewed interest in regionalization and localization' (7-8). multi-actor governance (Keulartz, 2005). Ruling authorities increasingly give societal interest groups and commeraccountability in the implementation of policy. There is, therefore, a horizontal hift from public and semi-public organizations towards more private arrangements and establishments (see figure 2 , bold line). This horizontal shift from the public to the private yields a relationship between government and citizens that cial parties responsibility and shared 
is no longer characterized by 'command and control', but more so by 'contract and negotiation' (see figure 2, striped line). The developments described in this paragraph could be seen in our view as a contextual turn which affects government communication.

\section{FIGURE 2}

Horizontal shift or multi-actor governance

(semi-)public

organisation

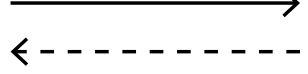

private arrangements and establishments

There are a number of perspectives on government communication, each relating to certain social developments, since government communication first arose in the Netherlands shortly after World War I. Below we describe three main phases of government communication that can be distinguished in our view, namely the phase of public information (2.2.1), the phase of dialogue (2.2.2), and the phase of the market or negotiation (2.2.3). These show that the character of government communication has changed: the contextual turn has resulted in emphasis on information being shifted to (social) interaction.

\subsubsection{Public information: Communication after the fact}

In the phase of public information, policy was first developed and then communicated to the public. In fact, up until the 1970s people did not talk of communication but rather of information services that explained and clarified policies already adopted (Eberg, 2006). This kind of communication focused on the dissemination of 'neutral' and objective information and amounted to communication 'about' policy. ${ }^{11}$ Policy was often experienced as elusive and a gap was experienced between the government and citizens. However, discussions on political democratisation arose in the 1970s and answers were sought to the questions: 'To what extent and how can, or rather how should, information held by the government be made available to citizens?' (Ministerie van Algemene Zaken, 1970:4-5). The government subsequently felt it to be its duty to inform citizens about government policy, thus resulting in quicker and more extensive
11 Fischer (2007) talks of about a rational model of decision making. In this model 'rational decision makers are seen to follow steps that closely parallel the requirements of scientific research. Decision makers first emperically identify a problem, and then formulate the objec tives and goals that would lead to an optimal solution. (...) Combining the information and evidence about probabilities, consequences, and costs and benefits, they select the most efficient, effective alternative $(223-224)$ dissemination of information concerning developments and themes that occupied government attention. Information was disseminated particularly through the news. Press conferences became fashionable and the government began cooperating more actively with various media. This ushered in the era of communication 'as' policy: in addition to publication and explanation communication also became an instrument of behavioural change (Jumelet and Wassenaar, 2003).

\subsubsection{Dialogue as an expression of socialisation}

Alongside the discussions on political democratisation that took place in the 1970s there was an increasing recognition and realisation that each city, each village and each neighbourhood had its own interest groups, atmosphere, political climate, and sensitivities. The Dutch government sought to take advantage of this by drawing interest groups into processes aimed at finding solutions for policy issues relevant to their interests as early as possible in the process of policy-making. Communication thus became something that was instituted prior to a policy. Jumelet and Wassenaar (2003) refer to this as communication 'preceding' policy. Citizens were given opportunities to voice their points of view prior to the government taking a decision. Or as Jumelet and Wassenaar (2003) put it: 'the citizenry is allowed to have its say in town halls, after which authorities take a decision' (25). In the late 1980s and early 1990s this expression of public involvement in policy development, i.e. socialisation (cf. Keulartz et al., 2004), was characteristic of government communication. The term dialogue is strongly associated with this period.

\section{TEXTBOX 1. Participatory Processes}

In response to the protests and critique of interest groups most governments have switched their approach from one that is centralist and top-down to one that is participatory. Fischer (2007) talks of participatory democracy. Participatory processes do have the potential to improve governance but they are not without problems. 'As soon as it became clear that the interests of many local interest groups would be substantially affected, the implementation process almost came to a standstill', Engelen et al. (2008:274) argues. Keulartz (2009) demonstrated a large gap between the rhetoric on participation and the real-life implementation of participatory processes. He also claims that, insofar as participatory processes are put into practice, the results are ambiguous at best. More specifically, Keulartz (2009) outlines five problems with participatory processes. First, the inclusion of a wide range of interest groups in the policy-making process is usually costly and 
time-consuming, as it could in turn delay the urgently needed adoption of policy measures. Second, the democratic quality of the consultation process involving local interest groups is questionable. The consultation process has more to do with bargaining than with arguing. Third, as it pertains to nature policy, participation falls severely short of the criterion of inclusiveness, because the fate of nature is actually placed in the hands of a minority who happen to live in the countryside. Fourth, too much emphasis on interactive policy will cause a situation in which politicians and policy-makers are held hostage by local interests at the expense of broader interests. Lastly, the increasing use of participatory processes can lead to serious output deficiencies. From both a quantitative and a qualitative perspective the shift from a top-down to a more bottom-up approach goes, according to Keulartz (2009), hand in hand with the debilitation of the original nature objectives. In a sense, participatory processes could take place at the expense of good nature conservation.

Dialogue requires a symmetric relationship between two parties. Both parties need each other in order to effectively analyse the situation and reach a consensus or draw a conclusion. Dialogue is therefore important to all the parties involved and the basic idea underlying dialogue is that of exchanging arguments in such a way that intersubjectivity and consensus are achieved (Burkart, 2009). Or, in terms of Ricoeur (1991): 'dialogue is an exchange of questions and answers (...) which directly connects the voice of one to the hearing of the other' (107). Language is a key factor because only by engaging in analysis and argumentation, and subsequently inferring conclusions, can one attain a cognitive understanding of an issue and subsequently develop the beliefs that allow for consensus (Habermas, 1989; Burkart, 2009). In language we can find a communal, rationally based definition of objective reality [truth: are the facts correct, is what I say true], the normativity of social reality [rightness: what is right in relation to other things], and the expressive components of the innerness of the actors involved [sincerity: am I
12 Rationality serves as a core concept for Habermas (1981). By definition, this cannot be held as equivalent to scientifically deter mined factuality, although de-mythologising is, for Habermas, a nec essary condition for rationality Raproviding good reasons for action or statements. The justification for these reasons may, in turn, be based on criticisable knowledge. I other words, rationality for Habermas calls for answerability to be demonstrated based on convincin arguments or claims. Furthermore, according to Habermas it mean that, with the exception of argumentation, all types of coercion are prohibited. Themes may not be excluded from potential rejection from a free dialogue or discussion and this dialogue is, in principle, accessible to everyone, It is important to note that power-free is not the same as influence-free. A dialogue is focused on change and in a sense influence is inherent to a dialogue. honest] (Habermas, 1989; Roothaan, 2005; Burkart, 2009). Truth, rightness, and sincerity are, for Habermas (1981), the rational assessment measures for weighing the validity of arguments. ${ }^{12} \mathrm{He}$ argues that truth, rightness, and sincerity are norms which are fundamental to all forms of conversation that aims to understand and agree; in this respect we also refer to validity claims. The provision is that this assessment takes place under symmetric communicative conditions that provide participants with equal opportunities to advance their opinions and deliver criticism. Habermas's (1981) aim was to arrive at a power-free dialogue for gaining consensus.

\subsubsection{Negotiation as a leading principle}

In this third phase there is a stronger call for negotiation than before (Keulartz et al., 2004). Arguments certainly remain important, but rationality as demonstrated by Habermas has now achieved a different connotation. Authors such as Latour (1993) believe that we construct reality in interaction with others. He talks of hybrid networks, a collective of networks that link people and caring for the meaning of matters. However, in a network context people do not always have clear-cut identities or preferences (Hajer, 2003). They are present and act in several and separate networks at the same time. People live within networks that 'stretch across territorially defined boundaries, and often without explicitly seeking representation in the sphere of formal politics in the location where they happen to live' (Hajer, 2003:88). Therefore Hajer (2003) argues that 'citizens could also be seen as political activists on 'stand by' who often need to be ignited in order to become politically involved' (88). It is mostly a 'public initiative that triggers people to reflect on what they really value, and that motivates them to voice their concerns or wishes and become politically active themselves' (88). So Hajer (2003) comes to the conclusion that public policy creates a public domain, 'a space in which people of various origins deliberate on their future as well as on their mutual interrelationships and their relationship to the government' (88) According to Hajer (2003) an intended policy intervention, for instance the implementation of the NEN, makes people aware

13 Hajer (2003) notes that in a of what they feel attached to, 'the awareness of what network context communities are unites them and what separates them from others' (89). based 'on the fact that their 'mem- Policymaking, he concludes, provides the practices in public policy programme' rather which people start to deliberate, 'policymaking $(. .$. than 'on shared normative beliefs' creates a sense of community and triggers meaningful (89). In this context he talks of a political participation' (89). ${ }^{13}$ 'The established thinking shared preferences. focuses on the issue of how to represent a (given) 
community and how to come to a fixed system of legitimate decision-making on policies' (96), but in a network context 'policymaking lead to the creation of communities that for themselves have to determine what constitutes a legitimate decision in a particular instance' (97). Hence he concludes that 'politics first of all [is] a matter of finding and defining the appropriate setting in which to stage the discursive exchange' (96).

\section{TEXTBOX 2. Implementation NEN}

Top-down implementation of the NEN faltered on local protests and according to Hajer (2003) 'the protests were not anticipated in the predominant neo-corporatist practices of consultation at the national level' (92). Consultation practices include a broad range of organizations, but according to Hajer (2003) the protests show that in a network context organizations are not reliable representations of feelings at the local level. In other words, landscape is part of the identity of people. Or, as Hajer put it (2003), landscapes are 'loaded with meaning and signifiers, stories and achievements' (93). So 'environmental policy should not be regarded as a fixed programme for ecological improvement that 'only' needs to be implemented, that politics was not merely a matter of doing 'more' or 'less' for the environment' (Hajer, 2003:92). Besides, for farmers in particular the plan for nature development 'indicated a lack of appreciation for the labour of previous generations' (93). In a sense, as Hajer (2003) rightly notes, the implementation of the NEN, i.e. the concept of nature development, ignores the farmers' way of life and also neglects Dutch history, that of an agrarian society that reclaimed land from water.

The situation described above differs from 'traditional' participatory processes in terms of focus and engagement (see Textbox 1). Interactive policymaking practices are typically employed before a formal political decision has been made, Hajer (2003) argues. But for a long time this was sectorally organised and content-oriented. Policymaking is increasingly moving away from 'sectoral orientation towards an integrated or area-oriented approach' (Hajer, 2003:94). In the network context interactive policymaking is not only a matter of content but also a matter of policymaking practices. 'It is the confrontation with a particular policy programme that first provides the shared basis for discussion, that first brings together the range of individuals in a particular region' (Hajer, 2003:95). So Hajer (2003) comes to the conclusion that policy discourse is constitutive for a region or what he calls a 'political community' (96). In other words, in a network context 'policy discourse can be constitutive of political identities' (89) while in the industrial or classical-modernist view 'policymaking is conceived of as the result of politics' (88).

As a result of the trends mentioned above, today interest groups and NGOs are autonomous and negotiation is the leading principle. The government has become a participant and fellow player or one of the negotiators in the marketplace. However, if the government is increasingly becoming a player in a process of negotiation and bartering, the question arises as to how we can obtain the best view of the government's own role and relationships with other players, such as interest groups or NGOs. In the Dutch nature policy this question is urgent because the government is currently inclined to hold social interest groups and other parties such as NGOs in the marketplace accountable for the implementation of nature policy. Furthermore a lot of conflicts have been noted during the implementation of the Dutch nature policy (see Introduction and Textbox 2). To answer the question just asked a closer inspection of communication is necessary.

\subsection{CLOSER EXAMINATION OF COMMUNICATION}

In the first part of this chapter we outlined a contextual turn. Distinct borders in terms of time are difficult to draw here. However, since the new millennium communication has been regarded as being central to the policy-making process in the Netherlands (Ministerie van Algemene Zaken, 2001). The fundamental perception is that we no longer need to attempt to translate policy into language that can be understood by citizens, but rather that policy as such must be comprehensible. Policy should itself be communicative (Jumelet and Wassenaar, 2003; Eberg, 2006). As such, policy and communication are seen to more or less merge into each other. Eberg (2006) regards government communication as the communal work field of both. Nowadays, we are increasingly seeing more attention being paid to communication as the modelling principle of an organisation and its relationship with society. This illustrates the shift from the instrumental role of communication in the sense of production and distribution of information to that of communication as a means to build and maintain relationships, in essence a shift from message to meaning.

The different forms and contexts in which communication plays a role make it a complex, as well as an interesting, phenomenon. Based on different assumptions about the character of communication, various theories have attempted to grasp this. 'Communication theory is enormously rich in the range of ideas that fall 
within its nominal scope,' Craig (1999:119) says. However, roughly speaking, there are two different main approaches when it comes to communication: the first approach emphasises the effect - an instrumental approach or functional vision; the second approach emphasises the meaning that originates between actors - a social approach or constructivist vision (cf. Van Ruler \& Verĉice, 2012). The instrumental approach of communication is rooted in the idea of the transfer of a message, i.e. the transmission model supposes an exchange of information (Carey, 1992). What is pivotal in this process is the information, understood as a kind of package, or its effect. Craig (1999) notes that the transmission model should at least be 'supplemented, if not entirely supplanted' (125) by a model that conceptualises communication as a constitutive process that 'produces and reproduces shared meaning' (125). He talks of the ritual approach to communication. This is not so much about conveying information as it is about shared beliefs. For authors such as Carey (1992) communication is dedicated to building and preserving communities and maintaining shared beliefs.

In this second part of this chapter we theorize our view on communication. The starting point is the question mentioned at the end of the previous part of this chapter. We briefly deal with the concept of argumentative turn to clarify the relationship between government and other parties (2.3.1). In argumentation 'language constructs' such as frames play an important role (2.3.2) and show depth layers that relate to normative concepts, leading to the conclusion that normative dimensions exist in communication (2.3.3). We argue that communication presupposes recognition of other people and their normative convictions (2.3.4). However, by communication the focus is on conveying meaning. For that reason we argue that communication is based on 'linguistic' interaction (2.3.5).

\subsubsection{Argumentative turn}

According to Fischer (2007) the 'postpositivist argumentative turn brings in the local knowledge of citizens, both empirical and normative, 'relevant to the social context to which policy is applied' (225). And although Fischer (2007) primarily focused on policy analysis, in his article he notes some aspects that could be interesting for the topic of our chapter. He asserts that the argumentative turn starts from 'a recognition that multiple perspectives are involved in the interpretation and understanding of social and political reality and the competing definitions of policy problems to which they give rise' (224). The argumentative turn focuses on 'the crucial role of language, rhetorical argument and stories in framing debate, as well as on structuring the deliberative context in which policy is made' (225). ${ }^{14}$
Fischer (2007) recognised the normative dimensions in the policy discourse. He argues that at the intersection where politics and science confront practice and ethics 'both policy analysts and decision makers would explore and compare the underlying assumptions being employed' (228). Therefore he advocates what he called a postpositivist approach. In his perspective this involves a different approach to empirical and normative inquiry. As he puts it:

Where conventional social science attempts to build in qualitative data about norms and values to an empirical model through quantification, the communications model reverses the task by fitting the quantitative data into the normative world view (Fischer, 2007:227).

In his view normative dimensions of policy questions cannot be dealt with through empirical analysis, 'that is, by converting them into variables to be operationalised' (227). Therefore he argues there is a need to seek 'a viable alternative by reorienting the task to begin from the normative perspective and fit the empirical in' (227). In his view normative analysis can be facilitated by an organised dialogue among competing normative positions.

In such a policy debate, each party would confront the others with counterproposals based on varying perceptions on the facts. The participants would organize the established data and fit them into the world view that underlines their own arguments (Fischer, 2007:227).

Fischer (2007) rightly notes that by doing so the locus of the interpretive process shifts from the scientific community to the practical world of the public realm.

He notes that the criteria for accepting or rejecting

14 With regard to government communication it is intriguing that Fischer (2007) notes that persuasion and justification play an important role in each stage of the policy process. 'Emphasizing the context-specific rhetorical of analytic practices - the ways the symbolism of language matters, the ways audiences needs to be taken into account, how solutions depend on problem construction, and forth - the argumentative approach recognizes that policy arguments are intimately involved with the exercise of power'(226) a proposal would be the same as those for accepting or rejecting a counterproposal and must be based on precisely the same data. As in interpretive explanation in general, he argues that the valid interpretation is the one that survives the widest range of criticisms. $\mathrm{He}$ talks of practical reason:

Practical reason holds that a decision depends on the person making it, and that formal rules of decision-making cannot be abstracted for persons and their actions into formal systems of demonstration modeled on deductive logic, as attempted 
by the methodologist of positivist social science. Reasoning refers here to a method for convincing or dissuading adversaries, and for coming

to an agreement with others about the legitimacy of a decision (Fischer, 2007:229-230).

Fischer (2007) argues that motives that have successfully undergone the test of argumentation can be seen as 'good reasons'. But it is interesting that he notes that, when seeking a decision on which action should be taken, 'a practical argument begins with the norms to which the participants in the controversy are committed and then seeks, by means of argument, to ground the decision on them' (230). This means, as he rightly notes, that practical reasoning supposes normative commitments.

Such norms are never universal or ever-lasting; all that is necessary in practical reasoning is that they be recognized by the audience (...) to whom the discourse is addressed at the specific time of the argument. Practical reasoning, as such, takes place among individuals or groups in a social context and in historical time. In contrast to the timelessness that is fundamental to deductive reasoning, the notion of temporality is essential to practical reasoning (Fischer, 2007:230)

In his view there is no unique way to construct a practical argument. According to Fischer (2007) data as well as evidence can be chosen 'in a wide variety of ways from the available information, and there are various methods of analysis and ways of ordering values' (230). To summarize, Fischer (2007) argues that the logic of practical reasoning is based on three principles: (i) it begins from opinions, values, or contestable viewpoints rather than axioms, (ii) it aims to elicit the adherence of the members of a particular audience to the claims presented for their consent and (iii) it does not strive to achieve purely intellectual agreement but rather to offer acceptable reasons for choices relevant to action. ${ }^{15}$

In argumentation as referred to above 'language constructs' such as frames play an important role. In the next section we elaborate on the concept of frames in greater detail and argue that thinking in terms of frames is helpful to gain insight into the depth layers in communication (see figure 3).
15 Fischer (2007) talks in his ar ticle of an intersection or complex blend between empirical data, normative assumptions, interpretative judgments inherent in the data-collection process, particular circum stances of a situational context and specific conclusions

\subsubsection{Frames}

16 The words selective and navigate are important in this respect. According to Entman (1993) framing is about 'selection and salience' (52) 'what to omit as well as include' (54) and 'attention to some aspects of reality while obscuring other elements' (55).

17 Although a frame gives direction and guidance to our thoughts, according to Balaban (2008) a frame exists by the grace of other frames: 'for each frame structure we have a complementary structure, i.e. the opposite' (12). Frame hould therefore not be interpreted as being static; they change over time (Hallahan 1999). Van Gorp (2007) notes that 'because a frame is characterized by some level of abstraction, so that it should be applicable to (entirely) different issues, it can be argued that an issue-specific frame, that is, a frame that is applicable only to one particular issue, in fact is preferably linked to another, more linked to another, more abstract
'master' frame' (67).

\section{TEXTBOX 3. Premises of Frames}

Van Gorp (2007) suggests six premises with regard to frames: (i) there ar more frames in a culture than we normally use, which ensures that there are different definitions that can be used and 'that the same events make different kinds of sense depending upon the frame applied' (63); (ii) because frames are part of a culture, 'the actual frames [are] not encompassed in media content'. The text and the frame must be seen as independent of one another. Both the attribution of meaning to media content and the connection with certain frames are part of the reading process' (63); (iii) because frames are related to cultural phenomena, frames are often invisible. 'Because these frames are often unnoticed and implicit, their impact is by stealth. (...) However, whether or not frames actually bring about individual effects depends on several factors, such as the receivers' degree of attention, interests, beliefs, experiences, desires and attitudes' (63); (iv) because 
frames are part of a culture, they are not the same as personal mental structures 'and probably not strictly individual frames' (63). He argues therefore that mental structures should be understood as schemata. 'The main difference between a schema and a frame is that schemata, defined as collections of organized knowledge, develop gradually, become more complex and are related to personal experiences and associated feelings (63) (v) frames are more stable than schemata and change only a little or gradually over time. This does not mean that frames are static, but rather dynamic in the sense that they depend on 'negotiation'. 'The application of frames is subject to negotiation: frames are contextualized by the journalis and the audience, new ones are selected and others may disappear without the frames themselves undergoing any change' (64); (vi) frames are part of what he calls social interaction. 'Media makers interact with their sources and other actors in the public arena, and the receivers interact with media content and with each other' (64).

Although it is possible to define what a frame is in conceptual terms, it is much more difficult to make it specific. Van Gorp (2007) proposes that 'frames seem to be everywhere, but no one knows where exactly they begin and where they end' (62). He rightly stresses that frames more or less exist independently of the individual and are part of a culture. ${ }^{18}$ Frames are 'in stock' in a culture and we use them consciously or unconsciously as we communicate, as well when we interpret the communication.

Lakoff's (2006) distinction between surface frames and deep frames is helpful in understanding how frames are related to normative dimensions in communication. ${ }^{20}$ Surface frames function at the level of our daily language and clarify what it is about and provide us with a point of view. In the case of surface frames it is their semantic meaning that is key; for ex-
18 He summarizes culture as 'an organized set of beliefs, codes, myths, stereotypes, values, norms, frames, and so forth that are shared in the collective memory of a group or society' (Van Gorp, 2007:62). 19 Entman (1993) mentions four functions of a frame: (1) defines a problem, (2) diagnoses causes of a problem, (3) provides a moral judgment and (4) offers a possible solution. In his opinion, during the communication process we once again see frames at four levels: (i) that of the sender, (ii) that of the text itself, (iii) that of the receiver and (iv) within a culture. 20 Van Gorp (2007) talks of frame packages, 'a cluster of logical organized devices that function as an identity kit for a frame' (64). Such a frame package is composed of three parts: 'the manfest framing devices, the manifest or latent reasonin devices and an implicit cultural phenomenon that displays the package as a whole. (...) My focus is on frame packages in which a cultural phenomeno functions as a central theme, such as an archetype, a mythical figure, a value, or a narrative' (64). What he interprets as 'framing devices' is similar to what we call 'surface frames' and what he calls 'reasoning devices' has to do with what we consider as 'deep frames'. ample the words 'dark wood' in the first place have a descriptive meaning relating to a certain type of wood and 'wilderness' refers to a rough place. Through their immediate meaning surface frames identify the context of the discourse. They are a sort of snapshot, Darnton and Kirk (2011) argue; they name the subject matter and at the same time provide an angle for viewing it. Surface frames could be seen as 'techniques' which structure communication. However, the frame 'dark wood' is easily associated with feelings of fascination, fear, initiation into a numinous reality, etc. The frame 'wilderness' usually has the connotation of a pristine and real nature, nature as it originally was before it was affected by humans. This illustrates that surface frames appeal to underlying values and convictions that can be communicated in deep frames and that ground our daily language in our (normative) convictions regarding the world and our lives. Deep frames 'are the evaluative context for the discourse' (Darnton and Kirk, 2011:75) that we need in order to interpret something as meaningful. Or, as Lakoff (2006) puts it:

Without deep frames there is nothing for surface frames to hang onto, slogans do not make sense without the appropriate deep frames in place (Lakoff, 2006:29).

To summarize, deep frames show our convictions and value patterns and could be seen as an articulation of worldviews. Naugle (2002) concludes that any explanation of the social and natural world is conditioned by worldviews, including our interpretation of and our relationship with nature. Worldviews shape our interactions with the environment, Peterson et al. (2008) argue. This is reflected in our communication about nature, especially at the level of deep frames, see figure 3 .

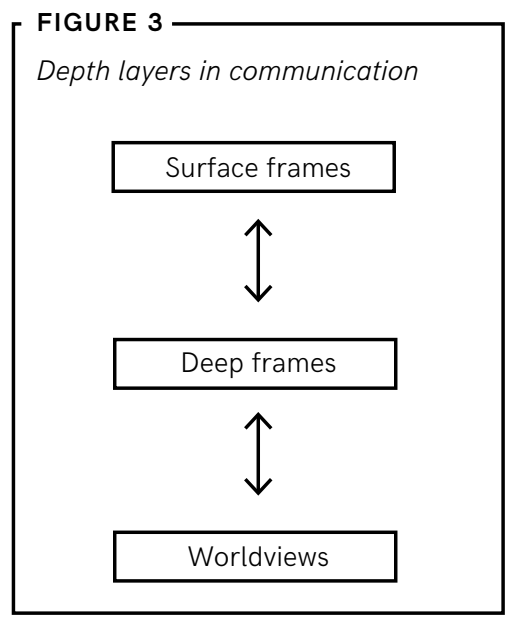




\subsubsection{Worldviews}

The relationship between deep frames and worldview becomes clearer when we look at what Naugle (2002) writes about worldviews. He considers worldviews as contextual phenomena that allow people to see things in a certain way and enable people to make connections between things. Worldviews emerge from a 'cultural milieu including religion, politics, science, place-based values, education and ethnicity' (Peterson et al., 2008: 707). All these things reshape worldviews constantly in an uncontrollable manner (Note et al., 2009). This makes worldviews a complex and comprehensive framework of one's basic beliefs about things. They define the person and provide people with 'fundamental assumptions upon which a life is based' (Naugle, 2002:291).

A Weltanschauung - as the primary system of narrative signs that articulate a vision of reality and lie at the base of individual and collective life - is the most significant set of presuppositions on the basis of which interpretations operates. One set of privileged signs - the worldview - provides the foundation and framework by which another set of signs - speech acts, texts, or artifacts - is understood (Naugle, 2002:313).

Worldviews function at the level of what Taylor (1989) refers to as 'frameworks of understanding' 'Frameworks provide the background, explicit or implicit, for our moral judgements, intuitions, or reactions (...)' (Taylor, 1989:26). As humans, we need these 'frameworks' to distinguish between good and evil, to know if something is important or not, if things are interesting or just trivial (Taylor, 1989). According to Taylor (1989) we can say that 'frameworks' (1) ensure that our moral reaction has the content we intend it to have and that they (2) provide the context necessary to determine the value, i.e. meaning, things have to us. In other words, to articulate these 'frameworks' is to explicate what makes sense, what is meaningful for us. In our view communication should be aimed at doing justice to all participants in the communication and therefore also to their worldviews as they become manifest in the frames they use in the communication. In others words, communication requires the recognition of each other's normative convictions on which our choices are based.

Interestingly, Taylor (1991) argues that things acquire their importance against the background of a pre-existing and inescapable horizon, as he calls it, with some things being worth it and others not so much or not at all, entirely prior to each choice. According to Taylor (1991) these horizons are given socially and historically. Choosing a certain normative position -Taylor (1991) talks about an ideal - presumes that there are other important positions in addition to one's own normative convictions. According to Taylor (1991) our own normative convictions cannot stand alone. It presumes a horizon that helps to define the issues that really matter. Only if I exist in a world in which 'significant others' matter can I define and place a value on my own normative convictions. With this reasoning in mind we need to consider what the consequences would be of recognising that there are different normative convictions. In other words, what it would mean to recognise the equivalence of different viewpoints. ${ }^{21}$

\subsubsection{Mutual recognition}

Taylor (1991) shows us that humans are dialogic beings. We are always interwoven with and connected to each other. It is precisely these connections that characterize our humanity. In fact, we could claim that human beings exist in relationships. Human beings cannot flourish without acknowledgement and appre-

21 Taylor (1991) argues with re- ciation by others; as humans we wish to be recognised gards to mutual recognition: 'To by others (Komter, 2003; Blokhuis, 2005). In thi come together on a mutual recog- section we want to explore what (mutual) recognition nition of difference - that is, of the equal value of different identities - requires that we share more than

belief in this principle; we have to share also some standards of value on which the identities concerned check out as equal. There must be some substantive agreement on value, or else the formal principle of equality will be empty and a sham. We can pay lip-service to equal recognition, but we won't really share an understanding of equality unless we share something more. Recognizing difference (...) requires a horizon of significance, in this case a shared one' (52).

22 Taylor (1991) argues that our identity is dependent on our relationship with so-called significant others. We know ourselves, but not in an immediate way. In answering the question of who we are, we depend on 'the detour of a story'. That story is formed in conversation with other (life) stories (Ricoeur, 1991). ntails. Or, as Berlin (2010) puts it:

I'm nothing if I do not find recognition (...) because also for me, I am how others see me. I identify myelf with the views of my environment: I feel myself somebody or nobody, depending on the position and function that I have in the social whole (Berlin, 2010:61)

Mutual recognition makes it possible to maintain relationships with others, and so in a certain way mutuality serves as a social structure. It not only estabishes relationships, but also stabilises them, according people flourish when they function in a variety of different social relationships and structures. In a sense we as humans are network-oriented by nature.

is an important insight in critical social theory that the interaction between people only flourishes when to Komter (2003). According to Van der Stoep (2006), 
people function in a variety of different social relationships, so that they are not locked up in one of the different social spheres and society is not ruled only by the law of the market, the law of the state, or the law of the family or church (Van der Stoep, 2006:143).

Each relationship presumes a unique form of reciprocity. It involves a transition: the human being (or organisation) that I am has undergone a change in the encounter with the other, the other has left their mark. In the interaction one's own uniqueness is retained, but at the same time both parties are changed and shaped.

\section{TEXTBOX 4. Discourse about Recognition}

The concept of recognition quickly establishes a link with the discourse about identity and multiculturalism. This is to do with the fact that (non-) recognition is linked by Taylor(1994) with 'causing harm to the other party'. In short, what it comes down to is that he believes that we need to 'recognise' each other; otherwise we will cause harm to the other party. This harm, according to him, is a form of 'oppression' and 'imprisoning someone in a false, distorted, and reduced mode of being', see quote below.

The thesis is that our identity is partly shaped by the recognition or its absence, often by the misrecognition of others, and so a person or group of people can suffer real damage, real distortion, if the people or society around them mirror back to them a confining or demeaning or contemptible picture of themselves. Non-recognition or misrecognition can inflict harm, can be a form of oppression, imprisoning someone in a false, distorted, and reduced mode of being (Taylor, 1994:25).

It is important not to confuse equality and equivalence. Recognition is not the same thing as 'everybody is equal in everything'. Recognition is navigating between acceptance of equivalence and being loyal to one's own individuality. It expresses a willingness to think about the importance of equal treatment in relation to one's own interest and the general interest. Recognition is in essence about taking other people seriously and regarding these people as worthy 'interaction partner(s)'. Recognition implies an authentic encounter between parties, i.e. with the aim of understanding the other party better (cf. Gadamer ([1975] 2004).

As mentioned in Textbox 4 we must not regard mutuality as a sort of reciprocity between equals. In our view it is a predominant misconception in communication that mutuality implies the equality of both parties. Mutuality does not imply equality. Quite a few communication philosophies approach communication as a symmetrical process. However, in our view, symmetry neglects social structures and the (civil) context too much. It is precisely the 'not being equal' that is the

23 By mutuality we mean interaction between parties. By asymme-

try we mean that in communication equality (symmetry) is impossible, because each party 'talks' from his/her own situated place and context. This applies in particular to the relationship between the government and interest groups. The government has a particular view on, for instance, land use and rural planning and sets the agenda with respect to the development of nature. The government is bound by its own laws and regulations and has to deal with interests that transcend topics and situations. It is important to note that equality is not the same as equivalence. Equality levels everyone's uniqueness and situatedness; by contrast equivalence emphasises that, despite differences, each contribution has the same expressive power. However, each contribution must be assessed on its merits and related to the overarching importance. In other words, the game of asymmetry is played on the basis of fundamental equivalence. Our calling this a game has to do with dependency: sometimes I need the other party and sometimes the other party needs me.

24 Language is not just a tool we use to express ourselves. We use language to achieve communication and concomitant content of $A$ meaning. Language has a structured nature of its own and at the same time it structures communication. Additionally, language has a creative and disclosing character. point of departure for recognition and that therefore of mutuality (ce. Blokh is, 2005$)$. If s in a sense asymmetrically to one another. ${ }^{23}$ This inequality characterizes our relationship to one another. To conclude, relationships, and in turn the commuication processes at their core, are mutual as well as symmetric. These mutual and sometimes asymmetric elationships pertain to interactions between communicating partners.

\subsubsection{Interaction}

In general we can define interaction as a process in which people (as individuals or as representatives of institutions or organizations ) act and react to others. It has to do with our response to the appeal of others. Or in terms of Goffman (1959):

Interaction (...) may be roughly defined as the reciprocal influence of individual upon one another's action when in one another's immediate physical resence. An interaction may be defined as all the interaction which occurs throughout any one occasion when a given set of individuals are in one anothr's continuous presence; the term 'an encounter' would do as well (Goffman, 1959:8)

According to Castells (2009), meaning can only be understood within the context of social relations in which meaning and information are processed. In a ense communication is sharing and disclosing, i.e. conveying meaning through interaction. Communi- 
cation could therefore be seen as interaction, in which language play an important role (cf. $\$ 2.3 .2$ ). Or, more philosophically: communication is disclosing meaning through language in interaction between human beings. ${ }^{24}$ Our approach to communication integrates the two main approaches that we mentioned earlier in this part of this chapter. Both approaches have their own point of truth. The functional approach justifiably draws attention to the aspect of language just as the constructivist approach rightly does to the aspect of context. However, both of them are to a certain extent restricted. We prefer a view of communication in which both aspects are interwoven. For that reason we approach communication as a primarily linguistic activity with meaning purposes.

\subsection{DISCUSSION}

In this chapter we theorize government communication with regard to the Dutch nature policy. Or, in other words, we examine in a conceptual manner to what extent new perceptions of relationships between government and other parties influence the discourse about nature policy in the Netherlands. In the first part of this chapter we have shown that a changing society affects government communication. In the Netherlands we have moved increasingly from information transmission or one-way communication on policy to a situation of negotiation. We have conceptualised this as a move from allocution to consultation, and we call this a contextual turn. This contextual turn makes the question of how to interpret the relationship between the government and, for example, NGOs an urgent one. We argue that to answer this question we need to be aware of the normative dimensions in communication. In the second part of this chapter we assert that it would be helpful to distinguish two levels or frames in communication in order to acquire a better understanding of these normative dimensions in communication. Surface frames function at the level of our daily language and identify the context of the discourse. However, surface frames appeal to underlying values and convictions that can be communicated in deep frames. Deep frames shows that our communication is grounded in normative convictions regarding the world and our lives, so called worldviews. This makes clear that any discourse is conditioned by worldviews, including the discourse about nature policy. So we claim that, without insight into the normative dimensions in communication, the discourse about the Dutch nature police cannot be properly understood.

So what are the implications of this for the discourse about Dutch nature policy? As mentioned in the introduction as well as in $\$ 2.2 .3$, the implementation of the NEN faltered for several reasons. At its core there is a gap between the rhetoric about participation and real-life practice; it is still an institutional way of conducting nature policy. Despite the fact that a broad range of stakeholders in a network context are consulted, organizations are not reliable representations of people's feelings at local level (Hajer, 2003). In a network context the practices of policymaking become more important as well as more difficult. Hajer (2003) notes that nowadays policymaking is moving towards an integrated, area-oriented approach. Because the NEN is implemented at a local level, this development opens up new perspectives for nature policy. In a network context it is increasingly a specific policy programme in a particular region that brings together the range of individuals and provides the shared basis for discussion. In this context Hajer (2003) talks of a 'community of fate', see FootNote 13 and Textbox 5 below. Communication by the government is about more than providing and obtaining information; it is the government's task to provide each interested party with the opportunity to present its point of view too.

\section{TEXTBOX 5. Responsive Democracy}

In this context we sometimes talk of responsive democracy. This means openness by the government to civil initiatives and it means transparent, accessible decision-making processes (Bekkers, 2001). The opposite of responsive democracy is representative democracy, which entails the delegation of power by society to elected officials. An example of responsive democracy is boundary organizations. Boundary organizations are essentially interest group platforms and user associations that operate in the interface between groups and communities with different interests and ideas (Keulartz, 2009). Boundary organizations can help policy-makers create and maintain good working relationships about practical matters. Obviously, they only have an impact if they show real interest in the problems and perspectives of all the interest groups, Keulartz (2009) argued.

Nature policy is to do less with carrying out national policy, i.e. the greatest common divisor, than it is to do with recognising the individual positions of the various participants in the discourse. Each of the participants has his/her own substantive role and normative position. In other words, awareness of the multiple perspectives in the discourse and of the underlying assumptions, i.e. the normative choices of the various actors, is crucial. Swart et al. (2001) argued that it is important to know the competing perceptions and views, otherwise 
the processes of (nature) restoration may easily stagnate. The concept of argumentative turn shows us that decision-making begins with making explicit the norms to which the participants in the controversy are committed (Fischer, 2007). The decision-making then seeks, by means of argument, to base the decision on these norms.

To conclude, problems with the implementations of the NEN are related not so much to the intended change in nature policy - a move towards a pro-active strategy, i.e. nature development - but rather, and at least as much, to an institutional way of conducting nature policy and a lack of openness about normative choices in the discourse. In order to gain (public) trust and facilitate the implementation of a controversial policy such as the NEN in a network context the government needs to be open about its normative convictions. At the same time the government needs to be sensitive to the normative choices of other parties and citizens. The concept of the argumentative turn provides tools to put this into practice. More than ever before the network context calls for an awareness that not every communication process is the same: multi-level and multi-actor governance require an awareness that each process has its own dynamics and orbital period. The role of communication professionals is no longer primarily to produce communication materials or to transmit content. Communication professionals are first and foremost a 'spider in the web', and they need to focus on 'bonding and bridging' by conveying meaning of the actions of the government. However, this chapter opens up other possibilities for future studies. This chapter presents an analytical structure, but more research is needed to explore which normative convictions are present in the discourse about nature policy in the Netherlands.

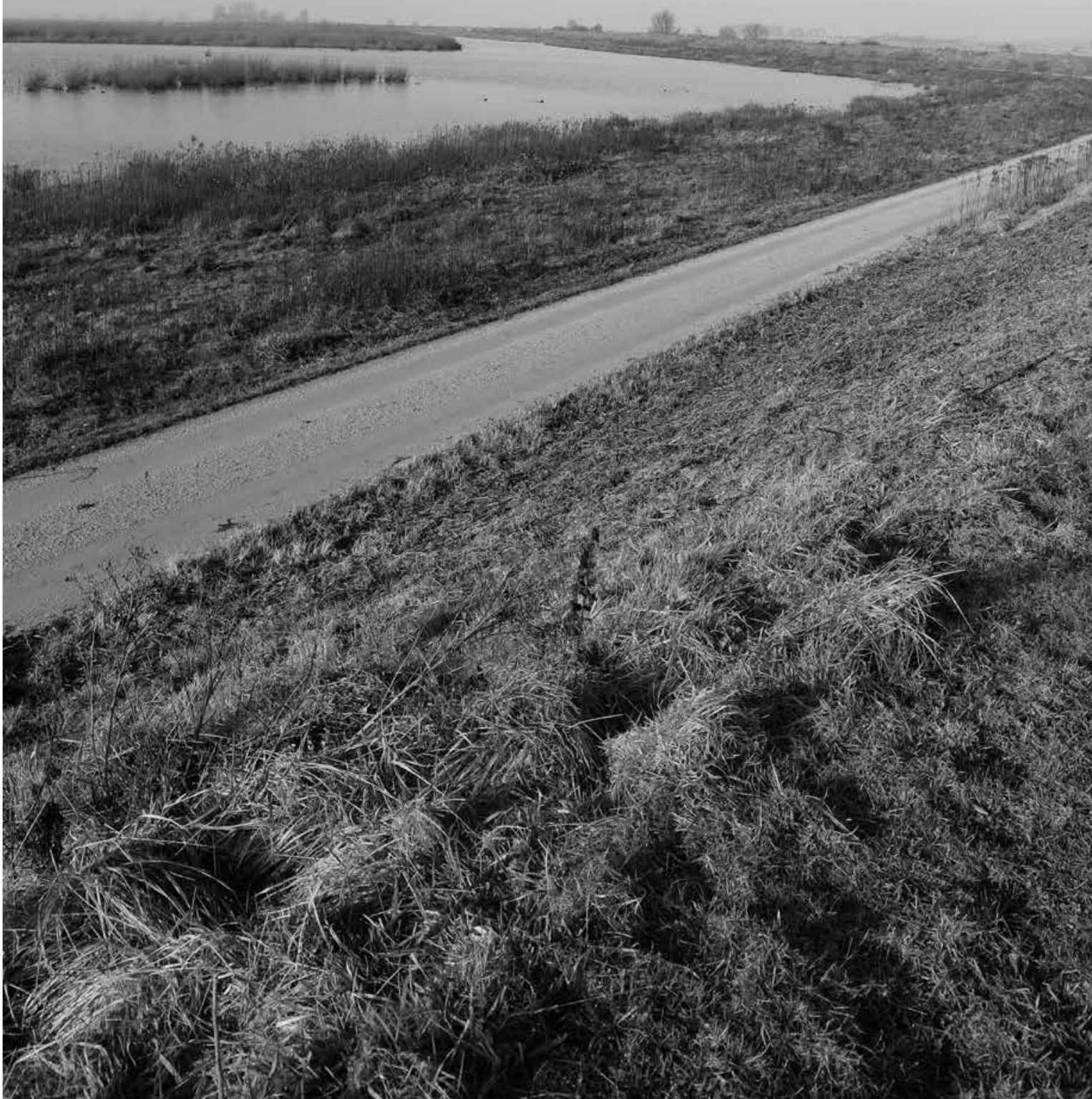




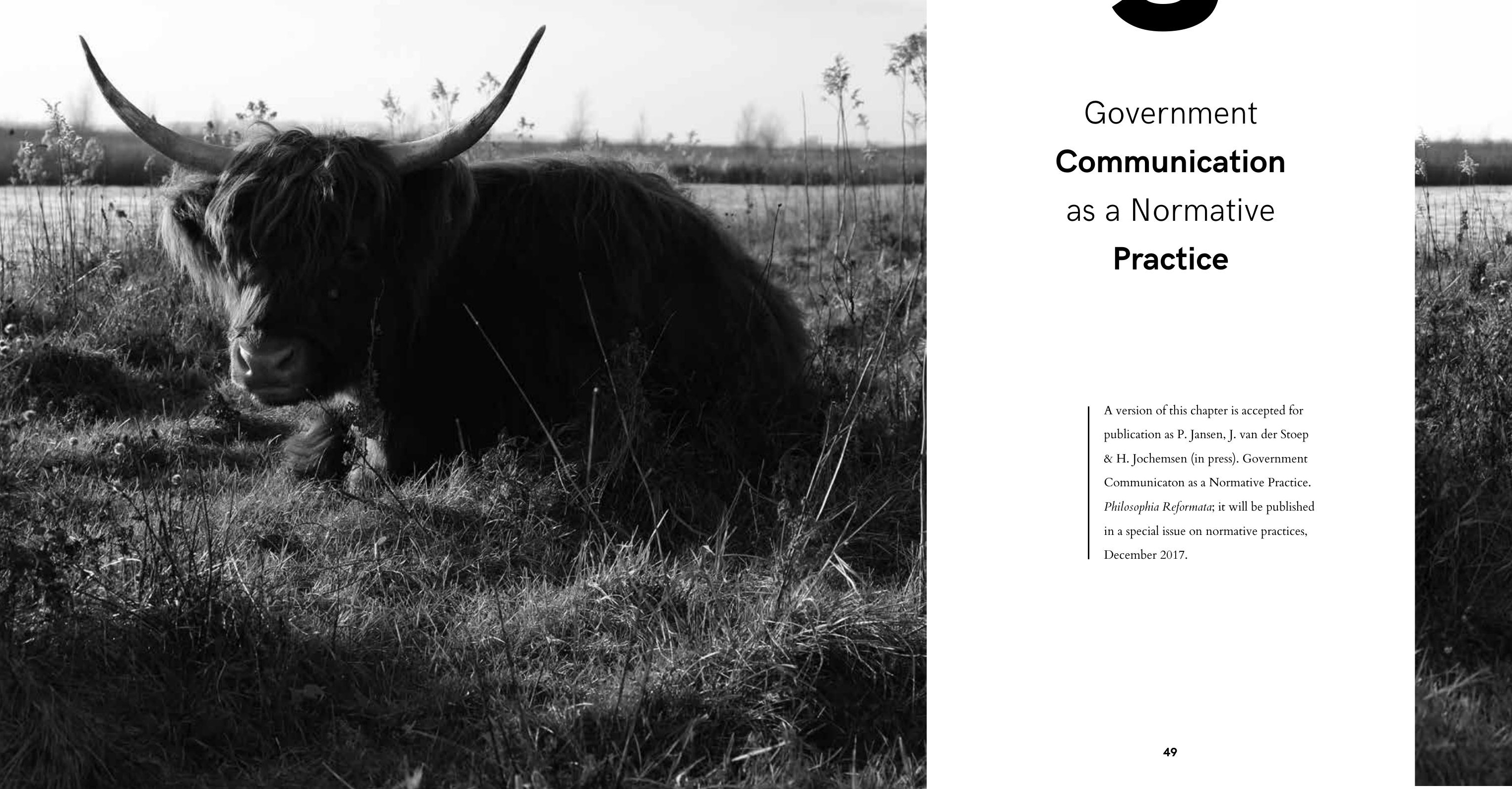




\section{Government Communication as a Normative Practice}

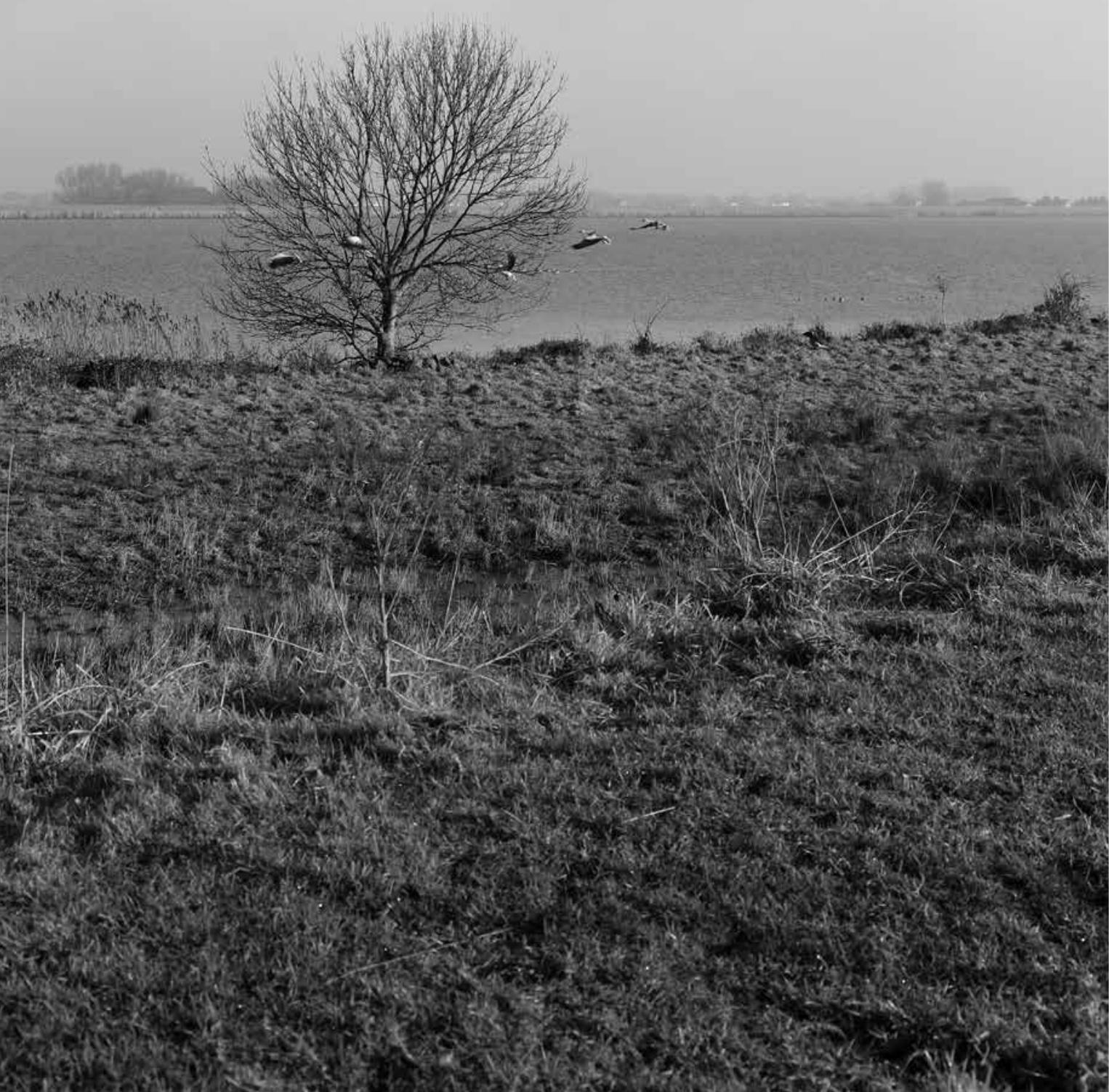

ABSTRACT - The network society is generally challenging for today's communication practitioners who are no longer the sole entities responsible for communication processes; this is a major change for many of them. In this paper, it will be contended that the normative practice model as developed within reformational philosophy is beneficial for clarifying the structure of communication practices. Based on this model, it is argued that government communication should not be considered as primarily an activity that focuses on societal legitimation of policy; it is, rather, an activity focusing on clarifying the meaning of the actions of the government. If the government can convincingly answer the question about the reason for their actions, societal legitimation will subsequently follow. Hence, it is argued that government communication is primarily linguistically qualified.

\section{INTRODUCTION}

Our network society requires that governments increasingly interact with their stakeholders. ${ }^{25}$ The predominant concept of communication has evolved from one-way communication to a situation in which each participant is simultaneously a sender and receiver (chapter 2). For that reason, Van Ruler \& Verčic (2005) speak about a paradigmatic change from a sender/re-

25 This chapter accords with Freeman (2010) who defines stakeholders as 'any group or individual who can affect or is affected by the achievement of the organization's objectives' (46). In the context of this chapter, this means that, when the word 'stakeholder' is used, citizens are included.

26 The literature speaks for that

reason about responsive democracy (Bekkers, 2001) and participatory democracy (Fischer, 2007). ceiver orientation (e.g., public information campaign that, in the Netherlands till 2012, were known as Postbus 51) to an actor orientation (e.g., participatory processes and co-creation of policy). In response to the protests and critique of stakeholders that they themselves are not involved enough in policy making, most governments have switched their approach over the past decades from one that is centralist and topdown to one that is participatory (cf. Fischer, 2007). ${ }^{26}$ In the Netherlands, this switch could be observed, for 
instance, in the implementation of a national nature network. ${ }^{27}$ Nature policy 'should not be regarded as a fixed programme for ecological improvement that 'only' needs to be implemented', Hajer (2003:92) argues. As a consequence, the consultation practices during the process of nature policy making and implementation currently include a broad range of stakeholders. Hence various parties with wide-ranging interests, ideologies, and visions about nature are involved in the achievement of a national nature network (chapter 4) which makes the implementation of it complex (cf. FootNote 57). However, it also indicates that stakeholders are progressively becoming co-creators and co-communicators of that (nature) policy (cf. Middel, 2002; Jumelet \& Wassenaar, 2003). This implies that government communication is not just received, but also discussed or even challenged..$^{28}$ The sender is never in complete control of the reception of the message. More precisely, stakeholders are increasingly not objects (of communication) but co-subjects and actors. This can generally be considered as a transition from allocution - where the sender determines what, when, and by which medium messages are communicated - to consultation in which the sender makes information available to a variety of public entities, and the receiver determines what, when, and by which medium that information should be accessed (chapter 2). ${ }^{29}$

The current situation entails a general challenge to today's communication practitioners. ${ }^{30}$ They are no longer the only entities responsible for organizational communication. For many communication practitioners, this is a major transition that involves a mentality shift. Instead of transmitting information, communication is currently more focused on communication as a two-way process for creating and exchanging meaning, preferably interactive and participatory at all levels (Servaes, 1999; Blokhuis,
27 An important element of the Dutch Nature Policy is the achievement network, before 2013 known as the Ecologische Hoofdstructuur (EHS). Within this national nature network, the conservation, restoration, and development of nationaly and internation systems are given shape.

28 In reformational philosophy, the state is generally a public legal community of government and nationals (cf. Zwart, 1994; Chaplin, 2007; Strauss, 2009). The need for communication is part of the well-functioning of this community. However, the focus is on the level of ministries, departments, etc. At this level, it is beneficial to make a dis nal communication, for instance between management and employ ees as well as external communication, i.e., communication with stakeholders (cf. FootNote 25). Cf. Glenny (2010) defines government communication as the apolitical and non-partisan communication of policy by the executive arm of government. This definition shows that government communication is about 'communication of policy'. With regards to the topic of this chapter, we would like to add the sentence 'in which communication practitioners are involved'.

29 'Being accessed' is also 'bein assessed': consultation implies an evaluative response, not just freedom concerning what, when and how to access the information.

30 In this paper, the term practitioner is preferred over the term professional. As Jochemsen (2006) argues, the term practitioner suggests that the (professional) practice is the primary focus.
2005). These changes have caused 'widespread uncertainty' in the context of 'who we are and where we should go with our profession' (Aarts, 2012:18). Is the communication practitioner of the government foremost a formal representative of the government equipped to defend their policy? Is this person the primary individual that relates information from outside the organization to the boardroom, or does this person create the preconditions for a situation in which the sharing of stories between the government and its stakeholder can occur? These types of questions create uncertainty about how to behave in a specific professional situation. However, it also affords an opportunity to think more deeply about the structure of the communication practice.

Based on the model of normative practice as developed within reformational philosophy, this paper intends to clarify the structure of communication practices. ${ }^{31}$ Because communication is always embedded in a certain context, delimitation is needed. The primary focus of this paper is government communication, as already explained in FootNote 28. Hence, an answer is sought to the question of whether the model of normative practice is beneficial for clarifying the task of government communication practitioners. This paper will first briefly present the model of normative practice in general. Second, this model is applied to government communication. Finally, the model of normative practice is discussed regarding how it can assist in avoiding confusion about the role of government communication practitioners.

31 Although several authors have written about the structure of com

munication (cf. Blokhuis, 2005), in our opinion, analysing (government) communication in terms of a normative practice model offers an important contribution to the discussion of this communication.

32 His full description is as follows: 'by a 'practice' I am going to mean any coherent and complex of socially established cooperative human activity through which goods internal to that form of activity are realized in the course of trying to achieve those standards of excellence which are appropriate to, and partially definitive of that form of activity, with the result that human powers to achieves excellence, and human conceptions of the ends and goods involved, are systematically extended' (MacIntryre, 1984:187).

\subsection{NORMATIVE STRUCTURE}

\section{OF PRACTICES}

According to the theory of MacIntyre (1984), in general, a practice is a socially established and complex (human) activity designed to work together to achieve the objective or destination of the practice..$^{32}$ For instance education, medical care, or in the case of thi chapter, government communication. This description, first, demonstrates that a practice presupposes an institutional framework and social embedding. A practice does not function in an isolated position. oining a practice implies that an individual participates in a social activity that already exists and is embedded in a broader social and historical context. The person becomes immersed in an ongoing tradition and 
becomes part of it - a practitioner becomes a 'carrier' of the practice, Reckwitz (2002) argues. ${ }^{33}$ Or as MacIntyre (1984) notes:

To enter into a practice is to enter a relationship not only with its contemporary practitioners, but also with those who have preceded us in the practice, particularly those, whose achievement extended the reach of the practice to its present point (MacIntryre, 1984:194).

Subsequently, a practice must have a common purpose. This suggests that the activities of a practice are not foremostly characterized by technical skills but instead intend to achieve the so called 'finality' of the practice, i.e., a reason or core value for which the practice exists - here we speak about the 'telos' of a practice (cf. Jochemsen 2006). ${ }^{34}$ MacIntyre (1984) speaks in this context about 'goods internal'; 'internal good' according to Van der Stoep (2011), is 'an inalienably [sic] part of that specific type of profession and cannot be achieved by professionals of another type' (6).

Finally, it is indicated that MacIntyre's (1984) definition is associated with 'standards of excellence' for achieving the 'internal good'. These 'standards of excellence', i.e., quality criteria, determine whether an individual is a good practitioner. In addition, a good exercise of a practice includes 'strive for improvement' in order to better achieve the 'internal good' or 'telos' of a practice. By participating in a practice, practitioners grow systematically in the capacities they need for realizing the 'internal good' or 'telos' of a practice and excel in it thereby continuously improving a practice.

Inspired by McIntyre's (1984) definition of practices, reformational philosophers developed the so called normative practice model (NPM). An advantageous distinction within reformational philosophy is the distinction between structure and direction (cf. Glas, 2012). The structural dimension refers to the aspects in which something functions and the order and coherence between these aspects. The directional dimension concerns our attitude towards the structural dimension. Applying this distinction, the NPM presents a (normative) model of practices. The
33 Jochemsen (2006) speaks about 'sort of initiation': 'the individual practitioner is initiated into the practice by learning a certain way of doing things. The practice shapes the behaviour of individual practitioners before they can begin to reshape the practice. The prac tice influences the way practitioners interrelate as well' (103).

34 Jochemsen (2006) speaks about the telos of a practice: 'probably the Aristotelian term telos suits best here. (...) The activities makin up a practice are directed at the realization of this finality, this telos of that actual practice. It is important to distinguish this finality from goals that individual practitioners may have. Goals set by individual or collective actors (...) do not necessarily contribute to the realization of the telos of (...) practice simply because one is practitioner. (...) The telos of a practice belong to the very nature of the practice and is not founded in the intention of the practitioner or client/patient/user' (103).

constitutive side or structural dimension (3.2.1) is about 'the architecture of a practice' (Jochemsen 2006,106) whereas the regulative side or directional dimension (3.2.2) relates to the practitioners' interpretation of the rules.

\subsubsection{Constitutive side or structural dimension}

Inherent to the concept of NPM is that rules or norms constitute a practice.

'Norms belong to the practice itself in an intrinsic way,' Glas (2012) argues, 'such that, when these norms are violated, the identity of

35 It is important to note that what is called norms here does not just refer to explicit norms or rules but also to implicit norms or rules of how to do something. Jochemsen (2006) relates in this context about the tacit character of rules, 'This means that rules can be followed even without a conscious decision of the practitioner at each moment they are applied' (106). He further contends that 'in his context, does not so much refer to rules in the sense of 'knowing that' which implies the ability to explicitly for- Hence, norms or constitutive rules can be considered mulate the applied rules. Rather, it as quality standards. In general, it can be conclud-
also includes knowing rules in the also includes knowing rules in the sense of ' rules are embodied in professional conduct consisting of the ability to act according to rules and to assess the correctness of this application even without $m$ plicit' (104).

36 Hoogland \& Jochemsen (2000) argue that, in every practice, a number of aspects can be distinguished. They define 'aspects' as follows: 'The concept of 'aspect' refers to an irreducible mode of human experience that at the same time constitutes a way of evaluation human activity, e.g., the performance of practices ( ) Each of the modal aspects had a core meaning that is a normative principle. These normative principles can be used as criteria in the evaluation of a particular performance of a practice' (464). the practice itself is threatened' (4). ${ }^{35}$ These norms or constitutive rules 'show a certain order and qualify the practice in distinct ways' (4). Otherwise stated:

These rules have an intrinsic normative nature in the sense that they prescribe a certain way of perorming a practice and at the same time constitute he possibility to evaluate the correctness of the actions performed within that practice (Hoogland \& ochemsen, 2000:463 that norms or constitutive rules 'make a practice recognizable as a specific practice' and this 'normative structure (...) can be considered the 'playing field' for concrete goals and actions within that practice' (Hoogland \& Jochemsen, 2000:464).

According to Jochemsen et al. (2006), three categories of norms or constitutive rules can be distinguished: qualifying norms, foundational norms, and conditional norms. The qualifying norms, first, are derived from the normative principle of the aspect that gives a particular practice its own typical character, the qualifying aspect' (Hoogland \& Jochemsen, 2000:465). ${ }^{36}$ They characterize the practice as it is and, therefore, are strongly related to the 'telos' of it. Everything that the practitioner does is directed towards this 'telos'. The other normative principles 'should be under the 
guidance of the qualifying principle' (Jochemsen, 2006:105). Interestingly, Van der Stoep (2011) argues:

Being a professional is a way of living that is worthwhile in itself, with its own intrinsic value. Professionals are not just driven by economic interests but also by the intrinsic good of the profession itself. (...) Their profession provides them with a sense of dignity and pride that goes beyond their material concerns (Van der Stoep, 2011:5)

Second, to achieve the 'telos' of a practice, professional skills and typical professional techniques are required. In these skills and techniques, a specific profession has its basis, 'The founding constitutive rules prescribe the activities that give a particular practice its characteristic content' (Jochemsen, 2006:105). This basis is normative according to him:

There is a need for action and this acting gets a specific form through pro fession-typical techniques. A practitioner needs to control them if he wants to be able to fruitfully join a practice (Jochemsen et al, 2006:11). ${ }^{37}$

Finally, conditional norms are those that must be observed in some way but do not inherently define the typical character of the practice. They define conditions that must be taken into account during the performance of a practice, 'but they neither define the 'technicalities' of the practice nor its finality' (Jochemsen, 2006:105). This involves norms that are a component of the broader normative framework, for instance, the economic and legal aspects. In the case of medical care, for instance, the economic aspect refers to 'efficiency and effectiveness of the use of health services in general and professional conduct in particular' (Jochemsen et al., 2006:31; cf. 43-44). In the same case, the legal aspects refer to the entitlement to information, the so called toestemmingsbeginsel (consent principle), rules about privacy, etc.

\subsubsection{Regulative side or directional dimension}

While the constitutive side describes the profession in its structure, the regulative shows 'in which direction the practice is moving, on the basis of values and beliefs (Jochemsen et al., 2006:12). In other words:
37 Jochemsen et al (2006) argue that each profession comes from what they refer to as a cultura impulse: 'something must be done to a recognized problem, a shared need or interest '(11). In response to this impulse, a certain action must be taken.
The constitutive side of a practice (...) embodies the normative constitutive principle and rules that should guide the performance of the practice and provide norms required to assess that performance. However, any performance and assessment involves a specific interpretation of the rules. (...) Such interpretation departs from a wider interpretative framework concerning the meaning of that practice for human life and for society and, hence, on the direction performances of the practice should have (Jochemsen, 2006:106). ${ }^{38}$

Values and beliefs 'reflect the worldview and, if they are religious, the religious beliefs of the people involved' (Jochemsen, 2006:107). Therefore, Jochemsen

38 Jochemsen (2006) argues that the values and beliefs with regards to the regulative side of a practice also function as a sort of reference point 'for a critical assessment of existing ways of performing practices by practitioners and of innovation and improvement of practices' (107). In his opinion, this is an important function of the regulative side because 'the fact that a certain community of practitioners accepts certain standards of excellence does not mean that those standards are the best possible' (107).

39 E.g. a liberal worldview does

not allow the government to be the moral judge of the behavior of its citizens with the exception of when the actions of one individual impose upon the interests of others. That may lead, for instance, 'to a governmental advocacy for use of condoms, but not for monogamy' (Klop, 1996:312). However, promoting the use of prophylactics, i.c. drocacy of condom use, implies certain values and beliefs. Hence, a policy on condom use illustrates that there is a directional dimension in government communication.

40 The term 'enkapsis' is used within the reformational philosophy to indicate cases where two or more different structures are interwoven in such a way that each retains its own internal structure.
(2006) argues that any performance of a practice is that 'there is no 'neutra' erformance of a practice' (107), and, 'depending on their view on the meaning and coherence of reality, people act differently in concrete practices' (Hoogland Jochemsen, 2000:466). ${ }^{39}$

\subsection{STRUCTURAL ANALYSIS OF GOVERNMENT COMMUNICATION}

In this section, the theoretical framework of normative practices is applied to government communication following the structure of the previous part of this paper. However, there is a preliminary question that must first be addressed, i.e., the question of whether communication is a practice. When thinking about this question, the first point to make is that communication does not exist as an independent practice. It is lways connected and subservient to other practice(s). In terms of reformational philosophy: communication is encaptically intertwined with other practices. ${ }^{40}$ However, communication is more than a set of skills and habits utilized in servicing other practices. As mentioned in the previous part of this paper, the concept of 'telos' is central to a practice as well as the idea that constitutive rules should be observed to achieve the 'telos' of it (cf. MacIntyre, 1984). According to this, it is contended that professionals engaged in activities aiming at the communication of their organ- 
ization with other actors are performing a practice because those activities have coherence and are collectively aiming at the realization of a value typical for communication. Otherwise stated, the practice of communication has its own 'telos', and certain constitutive rules must be observed in order to realize it. This second part of this chapter will seek the 'telos' of the governmental communication practice. The following elaboration of the normative practice model to governmental communication will begin with the constitutive side or structural dimension first (3.3.1) and subsequently discuss the regulative side or directional dimension (3.3.2).

\subsubsection{Constitutive side or structural dimension}

To trace the 'telos' of the communication practice, literature regarding the core of communication is examined. Research conducted by Jeffrey \& Brunton (2011) indicates that practitioners and academics define two superordinate goals associated with the practice of communication: strategically managing the communication process and managing relationships. ${ }^{41}$ This is illustrated as follows:

The first superordinate goal reflects the need for CM (= communication management) practitioners to manage all aspects of the communication process, including the integral role of the dynamic context in which the process occurs. This involves evaluating, controlling and using the communication process to achieve pre-determined objectives. As CM also endeavors to enhance the communication process with varied audiences, relationships with members of these audiences are also central. Accordingly, the management of relationships, the second superordinate goal, focuses on the participation perspective of the parties involved in the communication process. It is concerned with representing, understanding and advising members of both internal and external audiences (Jeffrey \& Brunton, 2011:65).

Tench \& Yeomans (2006) also emphasize the aspect of relationships with regards to communication. They conclude that 'public relations is about building and maintaining relationships' (290; cf. Carey, 1992). Communication is defined elsewhere more as a way in which people convey meaning (chapter 2). This raises the question of what primarily is the 'telos' (intrinsic meaning, purpose, etc.) of communication or, more precisely and in terms of reformational philosophy, how is (government) communication generally qualified? The next section will more structurally explore how government communication can be interpreted from a reformational philosophical perspective.
41 Their research shows that managing the communication process was evaluated by practitioners as being more important.

\section{Qualifying aspect}

In our view three (modal) aspects used within reformational philosophy play a special role: (i) the juridical aspect, (ii) the social aspect, and (iii) the linguistic aspect.

\section{(i) Juridical aspect}

As already mentioned above, communication is connected and subservient to other practice(s) including public policy. This indicates that government communication is a component of the broader practice of government actions. More precisely, government communication belongs to the practice of public policy that is institutionalized in government. Chaplin (2007) argues that the government is authorized by the motive of public justice ('telos'). In terms of the modal aspects as distinguished within reformational philosophy, this signifies that the government is juridically qualified and founded in the formative or historical aspect. However, the observation that government communication is serving the 'telos' of government does not rule out that communication by the government can simultaneously be perceived as a subpractice of its own - with its own 'telos'. Otherwise stated, the governmental communication practice in its entirety is

2 It is good to be aware of the guided by the general 'telos' of government, public connotation of the word 'social' justice; however, at the same time, it has an internal within reformational philosophy. In normativity of its own.

a broader sense it refers to human relationships which can have all

kinds of modal qualification, they (ii) Social aspect also are distinguished in relationships within a community or organisation, between individuals and between individuals (or communities and organisations) and communities or organisations. However, the term 'relationship' or 'relations' is rather complex within reformational philosophy and is also used aspects. All modal aspects in reformational philosophy imply relationships, the so called subject-subject and subject-object relations. For example, the linguistic aspect as such implies elations: people that speak with one another, and also lingual subject-object relation: the person that speaks about something.

Government communication does not occur in a isolated situation. As mentioned above, it always happens within the context of the government and is connected to other practices that form part of the policy process as well, e.g., relationships with political parties, the press, other departments, etc. However, government communication in itself serves the organization and is related to stakeholders. It can be stated that government communication takes places embedded in the social relationships within society between ministries, departments, etc. and stakeholders. However, in reformational philosophy, the word 'social' is hardly be used in this sense; the word 'societal' is more suitable in this case ${ }^{42} \mathrm{~W}$ ithin reformational 
philosophy, the basic point regarding the social aspect is more about the social intercourse between human beings or, as otherwise referred to, the social aspect within relationships. From the perspective of reformational philosophy, a more fundamental understanding of 'social' is that a human being 'radically fellow human being is' (Van Woudenberg, 1992:101). According to him:

The human being is at the root of his existence connected with his fellow man. It's not that the fellow-man first appears in the perspective of the modal theory and only then in the social aspect. No, the human race as human community consists of an entity structure containing, among others, a social aspect. 'Social' here doesn't carry the broad meaning of 'solidarity or humanity' but the one geared towards the meaning of omgang en verkeer (social intercourse). Here it's not about 'intersubjectivity', it's about 'interaction'. Within this interaction it's tact, politeness, amiability, courtesy etc. which is the norm (Van Woudenberg, 1992:101).43

\section{Or in terms of Strauss (2009):}

It is impossible to assume that human beings function first in some aspects and later in others. For example, it does not make sense to start a reflection about the nature of the 'social' by first contemplating a supposedly lonesome individual, for it belongs to the very constitution of every individual human being that it functions at once (simultaneously) within every modal aspect, including the social aspect. (...) A person is not an 'abstract individual' that has a 'social function' only in the second place.(...) Social interaction within human society gives expression to phenomena such as courtesy, tact, and politeness. Within this aspect, we find a point of orientation for a first classification of different kinds of social intercourse between human beings (Strauss, 2009:97).

It can be concluded that in reformational philosophy, the social aspect revolves around 'social intercourse' (cf. Dooyeweerd, 1969). However, does 'social intercourse' capture the core of (government) communication? We are of the opinion that it does not. Imagine two people sitting at a table who do not speak the same language. They can drink a beer and have a meal together, however, the communication is still basic. Perhaps someone
43 The word 'interaction' is easily misunderstood as if 'interaction' as such is 'social'. See previous FootNote: in reformational philos ophy, all modal aspects include subject-subject relation and from the physical aspect this implies interaction. In other words, human interaction is implied in all typically human aspects as they all include subject-subject relations.

gives a presentation. When the individual receives generous applause, does that mean that the audience has understood what had been said? What matters is that what the speaker would like to say is correctly understood, i.e., that the message is conveyed and the meaning is captured. Meaning is central in the linguistic aspect (Basden, 2008; Strauss, 2009). Therefore, although social norms play an important role, it is opined that communication is linguistic instead of socially qualified or, as otherwise stated: communication is a disclosed lingually qualified phenomenon, anticipating the social aspect (Strauss, 2009).

\section{(iii) Linguistic aspect}

Van Woudenberg (1992) contends that the linguistic aspect is about 'clarity'44, 'clarity is not the only, but the central norm for language' (98). ${ }^{45}$ However, when it comes to the use of signs and symbols, clarity is a precondition but not the reason of their use. Signs and symbols are used to convey meaning. Hence we believe that the normative principle of the linguistic aspect is meaning and that communication, being qualified by the linguistic aspect, is also qualified by meaning (cf. Basden, 2008; Strauss, 2009 - both speak about 'signification'). However, meaning takes shape between human beings in which social norms play a role. In terms of reformational philosophy: the linguistic aspect anticipates the social aspect. According to Van Woudenberg (1992), the linguistic aspect is not equal to the social aspect; in the use of language, the social is supplementary, i.e., 'adherent and not inherent' (101)

Taking into account the three (modal) aspects mentioned above, it was concluded that government communication in itself is linguistically qualified in which 'conveying meaning' can be defined as the normative principle, i.e., the 'telos' of the linguistic aspect. In government communication, the social aspect, as approached in reformational philosophy and consequently not used in a broader sense but in modal sense, is supplementary or even better: the linguistic aspect anticipates the

44 In a general sense, this also means intelligiblity, i.e., being able to use the proper grammatical rules' (Burkart, 2009:146) and a correct use of a profession's own erminology/jargon.

45 Cf. Strauss (2009, especially pages 320 - 341) for a more anaytical overview of communication from a reformational philosophical perspective. social aspect. Finally, the entire government communication practice is guided by public justice.

The conclusion above is beneficial when addressing the topic of societal legitimation, which is urgent in relationship to government communication (cf. Ihlen \& Van Ruler, 2007; Van Ruler, 2007). Societal legitimation appears to be considered as the primary goal of government communication. However, as mentioned 
previously, what matters in communication is that the message is correctly understood. Hence government communication should primarily be perceived as an (linguistic) activity that focuses on conveying the meaning of the actions of the government (cf. Middel, 2002; chapter 2). ${ }^{46}$ This means that the government should unambiguously express what they consider meaningful in relationship to the reason of these actions. Just as with other actors that communicate, the government has a reason for their actions regarding what they consider to be their core responsibility. If they can answer convincingly and truthfully about the reason for their actions (cf. Aarts, 2012; Van der Stoep, 2016), societal legitimation will subsequently follow. If not, the government loses their reliability and hence societal legitimation. This means that societal legitimation is an intended effect and not the primary goal. Otherwise stated, good communication means that all the parties are involved and treated in a correct manner and that the actions of the government are correctly understood. However, understanding something is not the same as agreeing with it, although social legitimation most frequently has these connotations.

\section{Founding constitutive rules}

In order to realize the 'telos' of the practice, professional skills and profession-typical techniques are required. The comprehensive knowledge and skills, in terms of the NPM founding constitutive rules, define the field of competence of the communication practitioner. This paragraph elaborates on these rules.

Professional skills and profession-typical techniques are often transcribed in socalled profession profiles which are composed of core tasks and levels. According to Logeion, the Dutch organization for communications professionals, six key tasks are important for communication practitioners: they must be able to identify and analyse (observe, identify, and interpret things); be able to advise and represent (represent the organization both internally and externally and provide advice about communication forms and strategy); be able to integrate (bringing together input from inside and outside the organization into a coherent story; 'the ability to adopt different strategies in an environment where new knowledge must not only be added but also replace the old' (Jeffrey \& Brunton, 2011:60); be able to create something (developing communication forms); be able to plan and organize something (working systematically and ensuring meetings); and, finally,
46 Middel (2002) argues that communication practitioner working in the field of the government has two tasks (or competencies as he calls it): confronting the government with the perspective of the outside world and attributing meaning to existing information provided by government. be able to supervise and manage communication processes in general (improving communication skills of people and leading and monitoring communication processes). ${ }^{47}$ To perform these six tasks, several competencies are required. According to Jeffrey \& Brunton (2011), competencies could be considered as 'the underlying foundational abilities that are integral to successfully carrying out the tasks and responsibilities and thus remain a stable blueprint for practice over time'(60).48 When asked to rate the importance of the competencies required to attain the two superordinate goals of the communication profession mentioned above, Jeffrey \& Brunton (2011), drawing from their quantitative research, conclude that six major areas emerged. The first is 'managing stakeholder relationships' which 'involves using communication to foster and maintain diverse, but significant, relationships' (67). Second is 'managing the external interface' which entails 'the ability concerned with managing the client's appearance in, or interaction with, the external environment, from dealing with a crisis to presenting their client's reputation in the best light, often in the media' (67). 'Socially responsible communication' and 'evaluation management' are rated as equally important in the third and fourth places. 'Evaluation management is the ability to plan, measure, monitor, and evaluate all aspects of the communication process while socially responsible communication is concerned with sustaining ethical and acceptable behavior by all stakeholders' (67). In fifth place, 'environmental monitoring', is 'actively monitoring the environment for early detection of changes or trends which may impact on key stakeholders' (67). Finally, in the sixth and last place, 'lobbying' is 'concerned with strategically advocating or lobbying for a particular point of view with both internal stakeholders and public policy groups' (67). Jeffrey \& Brunton (2011) noted that the first five competencies, 'although able to be ranked, were relatively close in importance', however, lobbying 'was rated as being much less important' (67).

47 See http://www.logeion.ny/

beroepsniveauprofielen (Dutch).

48 Based on research conduct- The question arises which of the above competencies ed in the UK, Jeffrey \& Brunton for communication practitioners are relevant in the (2011) note that communication field of government communication. Although the practitioner practioners must also und in order to improve and develop in mind as mentioned in the introduction, they probpractice. 'As the very nature of being 'professional' assumes knowledge under-pinn and abilities, then professionalism must be judged according to the ability to achieve a particular standard in those skills and abilities' (60). ably become more important, especially the first (manging stakeholder relationship), the fourth (evaluation management), and the fifth (environmental monioring). In addition, at the end of 2015, the Dutch Government published a document with the basic 
principles of their communication to ensure that all departments communicate according to the same principles. In summary, the Dutch Government defines the following six general principles as mentioned in Textbox 1.

\section{TEXTBOX 1 Basic principles for Government Communication}

1. Ministries will observe a number of basic principles in their communications with the press and public. These principles are entirely related to the Dutch constitutional system and the relationships within it between government and opposition and between government and the citizen.

2. Government communications must satisfy the citizen's entitlement to communication with and information from central government and the support of good democratic governance. Government communications will always be recognizable as such, relate to policy and organization and not be aimed at the personal image building of members of government and of other government organizations.

3. The government will actively bring its policy and the reasons and considerations underlying it to the attention of the widest possible audience and will always specify the policy phase to which the information relates. At an early stage, it will be indicated whether the government wishes to involve citizens in the preparation of plans and measures and, if so, then how.

4. The government will ensure that it can be easily contacted by the citizens. Furthermore, the government will respond quickly and adequately to requests for information and to complaints. Communications will be truthful, sufficiently accessible both in terms of technology and content, understandable, timely, and as focused as possible.

5. Communications 'overkill' will be avoided at all stages of the policy process. In principle, the government must not employ so many means of providing information that an imbalance arises with respect to the possibilities of other parties.

6. Central government communications will not become interwoven with party political interests.
The constitutive side of a practice includes conditional rules: norms that must be brought to fruition in some manner but which have no defining meaning for a specific practice. They are only supportive to the 'telos' of government communication. The next section will elaborate the conditional rules with regards to government communication.

\section{Conditional rules}

Based on the reformational philosophy, the following conditional rules or norms can be defined (cf. Cliteur, 1983; Van Woudenberg, 1992; Basden, 2008; Strauss, 2009): the sensitive or psychological aspect involves empathy, i.e., being aware of the emotions in society, denoting them, and addressing them proactively. This is illustrated by the refugee crises: feelings of fear, anger, grief, etc. prevail. A government communication practitioner can be expected to recognize these signals and act empathically but also with due objectivity. ${ }^{49}$ The logical-analytical aspect involves discernment: think and reason with conceptual clarity and also be able to distinguish fact from fiction and primary from secondary issues, etc. However, it also involves a certain degree of professional rationality. As illustrated above, government communication is often accompanied by emotions, and a government communication practitioner must be able to discern this, however, the outcome should not be determined by these emotions. The economic aspect primarily refers to efficiency and effectiveness in communication: possessing efficient communication tools for the different types of policy areas and their stakeholders; not using more words and images than necessary; and ensuring that the materials that are used achieve the intended results. The issue in this aspect is communicative frugality (cf. Strauss, 2009). Second, it also refers to the use of finance: practitioners earn a living with government communication, however, this is paid by public funds which requires prudent behavior. The aesthetics aspect is about harmony. This not only involves a beautiful layout and well-constructed communication expressions but also the tone of voice, i.e., is it appropriate and affecting. The legal aspect indicates that, in legislation, policy, and gov49 Tench \& Yeomans (2006) ar- ernance, the government should intend to do justice gue that it cannot be denied that
public relations people have to to the different stakeholders in their several positions justify the decisions and actions and responsibilities. This is also relevant in the field of of their organization to a range of
publics' (290). According to them, government communication regarding the 'balance 'They should have, therefore, an of interests': communication that is unilateral but does acute awareness of what their justice to each interest (cf. Verkerk, 2014). Further, publics' likely reactions will be and
whether there will be a sense of according to Glenny (2010): moral outrage or approval' (290). 
Laws covering freedom of information, privacy and whistleblowing 'affects the transparency of the executive branch of government' and lay obligatory standards for the acceptable conduct of government communication Glenny, 2010:1).

There is also the moral aspect. Krabbe (2011) concludes: 'The old adage of public relations in the Netherlands is 'open and honest' '(61). However, at the same time, he states:

Communication practitioners probably don't lie roundly and flatly, but openness is hard to find and misleading is common (Krabbe, 2011:61).

This aspect is related to what was stated above regarding reliability in relationship to societal legitimation. Finally, there is the pistic aspect that involves trust. ${ }^{50}$ (Public) Trust is closely related to societal legitimation and central to the practice of government communication. However, trust is not an independent goal but an intended effect of, again, convincing and reliable communication about the why of governmental actions (cf. De Vries, 2014).

\subsubsection{Regulative side or directional dimension}

The regulative side concerns the values and beliefs of the practitioners that often implicitly play a role in relationship to the practice. Within a profession or organization, there can be consensus about certain values and whether or not they are recorded in a code of conduct (e.g., Stockholm Accords). However, there are also values and beliefs related to an individual's personal life or those that are embedded in the culture (Jochemsen et al., 2006). For many years, the so called instrumental approach was dominant concerning government communication. Until the 1970s, people did not speak about communication but rather of information services that explained and clarified policies that were already adopted (Eberg, 2006). This type of communication focused on the dissemination of 'neutral' and objective information and amounted to communication 'about' policy. However, policy was often experienced as elusive, and a
50 Krabee (2011) states that trust is an overarching value of communication that is carried by other values and principles such as: honesty, reciprocity, autonomy, tolerance, caring/ servanthood, visibility, utility, dialogue, deliberation, etc. Tench \& Yeomans (2006) note that trust usually includes words such as reliability, confdence, faith, and integrity. They further argue that trust depends, to a large extent, on the integrity of the individual practitioner: 'if (...) practitioner acts ethically and professionally they are likely to be trusted. They will be described as having integrity - there is something wholesome, honest, an trustworthy about them. Being eth ical and professional is core to hav ing a good reputation' (290). gap was experienced between the government and its stakeholders. In response to the protests and the critique of interest groups, most governments switched their communication approach from one that was centralist and top-down to one tha is participatory. Interestingly, Hajer (2003) argues that 'the established thinking focuses on the issue of how to represent a (given) community and how to come to a fixed system of legitimate decision-making on policies' (96). However, he continues that, in a network context, 'policymaking leads to the creation of communities that for themselves have to determine what constitutes a legitimate decision in a particular instance' (97). Hence he concludes that, currently, 'politic is, first of all, a matter of finding and defining the appropriate setting in which to stage the discursive exchange' (96). This is in accordance with Fischer's (2007) observations. With regards to policy, he discusses an 'argumentative turn' that is rooted in postpositive thinking and brings in the (local) knowledge of stakeholders (esp. citizens), both empirical and normative, 'relevant to the social context to which policy is applied' (225). It focuses on 'the crucial role of language, rhetorical argument, and stories in framing debate, as well as on structuring the deliberative context in which policy is made' (225). The word 'framing' is crucial in this context. According to Van Gorp (2007), frames exist more or less independently from the individual and are an element of a culture. They lead a person's thoughts and discourses by presenting the world in a particular way, and they are used consciously or unconsciously as we communicate as well when we interpret the communication. Such framing entails an interplay between surface frames and deep frames (Lakoff, 2006; chapter 4). Surface frames function at the level of our daily language. For example, the words 'dark forest' first of all have a descriptive meaning for a certain type of woodland and 'wilderness' refers to a rugged place. Through their immediate meaning, surface frames identify the context of the discourse. However, these surface frames appeal to underlying values and convictions that can be communicated in deep frames and ground our daily language in our normative convictions regarding the world and our lives (chapter 4).

We see that regulative views can be expressed in the form of (deep) frames. It is important to explicate predominant regulative ideas and discuss them in society and within a profession. A practitioner in the field of government communication must be aware of deep frames in the communication with stakeholders. Stated differently, each of the participants has an individual personal position. Awareness of the multiple perspectives and the underlying assumptions, i.e., the normative choices of the various actors, is crucial. With no insight into these normative choices, a discourse about policy cannot be properly understood. This implies that the government must also be open about its normative convictions (cf. Textbox 1, Principle 3). 


\subsection{DISCUSSION}

This paper began by arguing that the network society supposes a changing relationship between government and its stakeholders that creates uncertainty among practitioners about how to behave in a specific professional situation. The view expressed in this paper is that the NPM clarifies the structure of communication practices and assists the practitioner by reflecting on the core value(s) of communication practices. The clarity achieved in this manner may decrease uncertainty. Normative aspects in which practices function and that must be brought to fruition to ensure that the practice functions adequately can be identified and named with the use of an NPM. Distinguishing a constitutive and regulative side is beneficial for differentiating between the constellation of normative principles of a practice (structure) and the guiding beliefs (direction) as well as for formulating the significance of each for a correct understanding of the practice. ${ }^{51}$ In other words, the NPM clearly constructs the framework that defines a specific practice and assists in thinking systematically about competencies that are required for a practitioner and also challenges this person to reflect on individual beliefs in relationship to that practice (cf. Jochemsen et al., 2006). ${ }^{52}$ It is important to note that a practice is, foremost, aiming at the pursuit of its 'good' ('telos') rather than striving for success. All things in which the 'good' is sacrificed for (personal) success violates that practice.

However, characteristic of the current times is a lack of common values and beliefs. As a result, it is more difficult 'to realize the load-bearing coherence of the institutions' (Boutellier, 2011:29). Boutellier (2011) wonders how, despite the loss of traditional religion and moral frameworks, there is still belief in an ordered society. ${ }^{53} \mathrm{He}$ concludes that the absence of a coherent moral structure does not automatically lead to disorder. According to him, individuals are able to perfectly establish order in an improvising manner, however, not in a traditional sense specifically based on shared values and beliefs but more centered on societal relevant features or themes (cf. Hajer, 2003; Broer et al., 2017). This raises the question of whether common ground can currently be determined more at the constitutive
51 Because these norms or con stitutive rules also function as quality standards, Hoogland \& Jochem sen (2000) argue that NPM can serve as a basis for integral quality management for a practice.

52 According to Hoogland Jochemsen (2000), a beneficial practice is impossible if practitioners do not reflect on their regulative ideas that direct the particular practice. 'It is part of the character of normative practices that they can only be 'opened up' by regulative ideas about the meaning and structural coherence of human experience' (466).

53 Boutellier (2011) distinguishes two perspectives with regards to regulation: (i) 'moral coherence' and (ii) 'institutional organization of society.' About the first, he wrote: 'the moral perspective refers to the meaning that others have to us, to our self-image, to our intuition about the good life (...) and about what we perceive as evil' (28) side (a perspective on what a certain practice should be aiming at) rather than at the regulative side of a practice (the guiding control beliefs of the practitioners). Stated in this manner, this question seems to neglect the relationship that exists between both sides. The constitutive and the regulative sides of a practice are indeed inextricably intertwined, however, they are distinguished at the same time. Inherent to the concept of the practices is that the very concept ensures 'supporting cohesion'; it provides a common ground, i.e., shared ideas about how to act and cross-links between practitioners with different value systems (cf. Broer et al., 2017).

It is argued in this paper that societal legitimation is crucial for government policy. However, this paper also contends that societal legitimation should not be the purpose of government communication. As discussed, the linguistic aspect is central to government communication that is about conveying the meaning of the actions of the government. If the government can convincingly and truthfully answer about the why of their actions, societal legitimation will subsequently follow. Stakeholders (esp. citizens) expect that the government communicates in a convincing and truthful manner which implies a positive response to the next three questions. A negative response to one or more of these questions will result in loss of reliability

54 Jacobson \& Kolluri (1999) formulated four questions in order to evaluate participatory communication, i.e., evaluate the interaction between 'experts' and either an individual or group: '(i) is the communicator's communication correct, that is, is the information being offered undistorted and reliable?, (ii) is the communicator's role legitimate given his or her role and the participation of other interested individuats, social groups, agencies, and nations who are party to the process of development?, (iii) is the communication offered sincerely in good faith without being $m$ on the part of the individual or any organization from which the individual may have been sent? and (iv) is the communicator's communication comprehensible to others, that is, $P$ are idiom, cultural factors, and/or message design adequately accounted?' (274). In other words, it is about Practical reason holds that a decision depends prehensibility (cf. Habermas, 1981). on the person making it, and that formal rules of 
decision-making cannot be abstracted for persons and their actions into formal systems of demonstration modelled on deductive logic as attempted by the methodologist of positivist social science. Reasoning refers here to a method for convincing or dissuading adversaries, and for coming to an agreement with others about the legitimacy of a decision (Fischer, 2007:229-230).

He contends that motives that have successfully experienced the test of argumentation can be considered as 'good reasons'. When seeking a decision on which action should be taken, 'a practical argument begins with the norms to which the participants in the controversy are committed and then seeks, by means of argument, to ground the decision on them' (Fischer, 2007:230). By doing so, intuitions about (public) justice are strengthened and contextualized. However, there is an inevitable moment of choice: intuitions must be weighed, ${ }^{56}$ placed along the yardstick of governments 'telos' whereby, subsequently, a decision must be made - 'this we want to communicate as government in this particular situation.' Hence what is (public) justice is not only an outcome of a majority rule process. Stakeholders play an important role, however, the government has its own responsibility which is defined by their 'telos'. Stated differently, at its core, government communication is for the benefit of government' 'telos'. If government communication practitioners work in such a

way, they can contribute positively to the coherent and convincing communication of the government with a reduction of confusion about their own role as a result. To conclude, in our opinion, the model of normative practice is helpful in clarifying the task of government communication practitioners. Otherwise stated, the model of normative practice clarifies what the 'telos' of a government communication practice should be. This may be important after a transition from 'allocution' to 'consultation' as described at the beginning of this chapter because it helps practitioners find their focus, for instance, between the wide-ranging and mostly conflicting interests and visions about nature that are involved in the achievement of a national nature network. ${ }^{57}$ It is expected that, as a result, there will be decreasing confusion about how to act behave in specific professional situations and increasing democratic decision making processes.
55 Tench \& Yeomans (2006) argue that it cannot be denied that public relations people 'have to justify the decisions and actions of their organization to a range of publics' (290). According to them 'they should have, therefore, a acute awareness of what their publics' likely reactions will be and whether there will be a sense of moral outrage or approval' (290). 56 Cf. Verkerk (2014), he speaks about 'justified interests'.

57 Aarts et al. (2015) refer to Van Bommel (2008) and De Vries (2014) for in depth analysis about implementation conflicts respec tively at de Drentsch $A a$ and the Wieden-Weerribben. They also argue that implementation conflicts are still the case in the Netherlands, but according to them 'a little is known about the character an progress of the actual discussions about nature' (Aarts, 2015:8

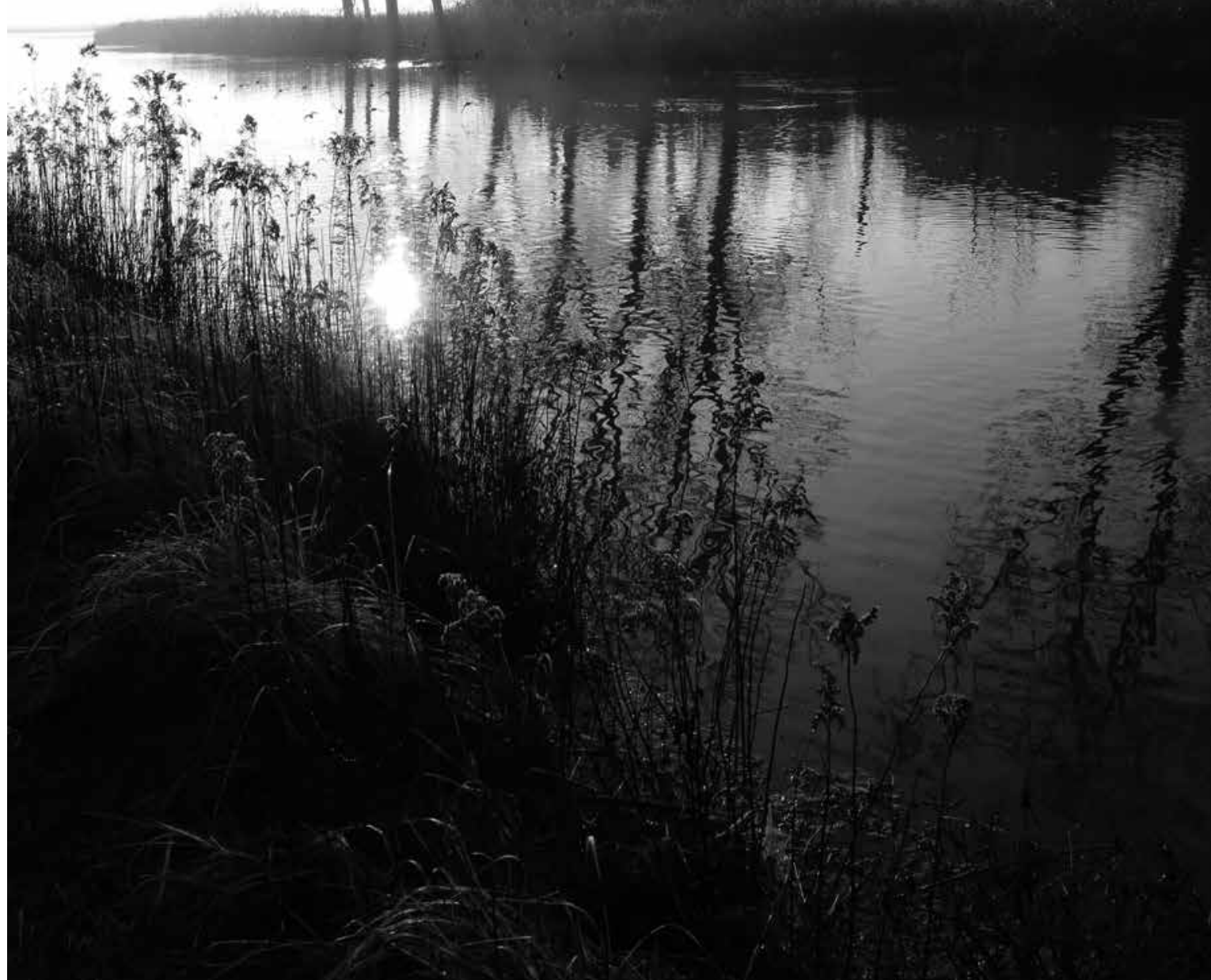



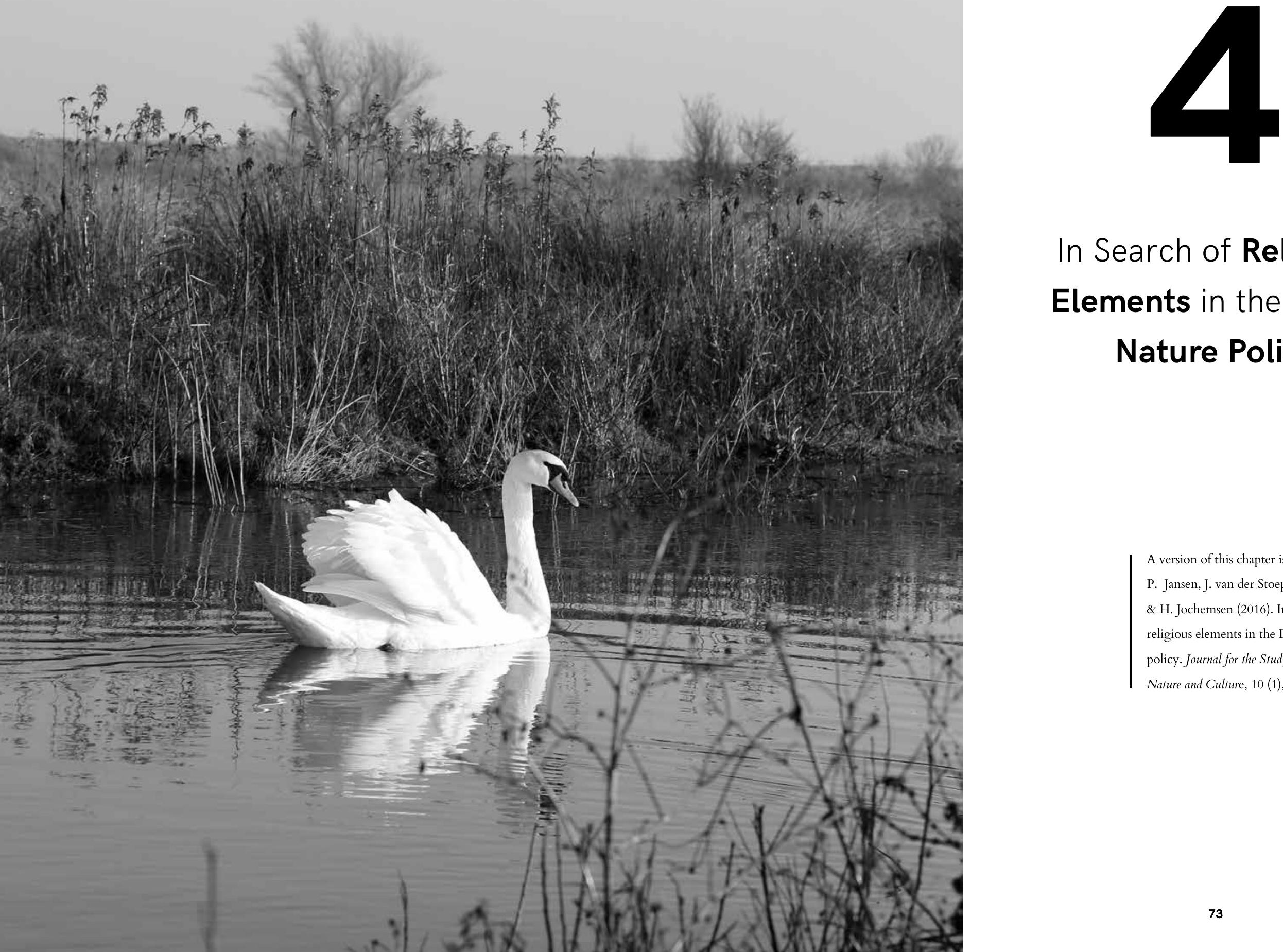

In Search of Religious

Elements in the Dutch

Nature Policy

P. Jansen, J. van der Stoep, J. Keulartz

\& H. Jochemsen (2016). In search of

religious elements in the Dutch nature

policy. Journal for the Study of Religion,

Nature and Culture, 10 (1), 91-116. 


\section{In Search of Religious}

\section{Elements in the Dutch}

Nature Policy

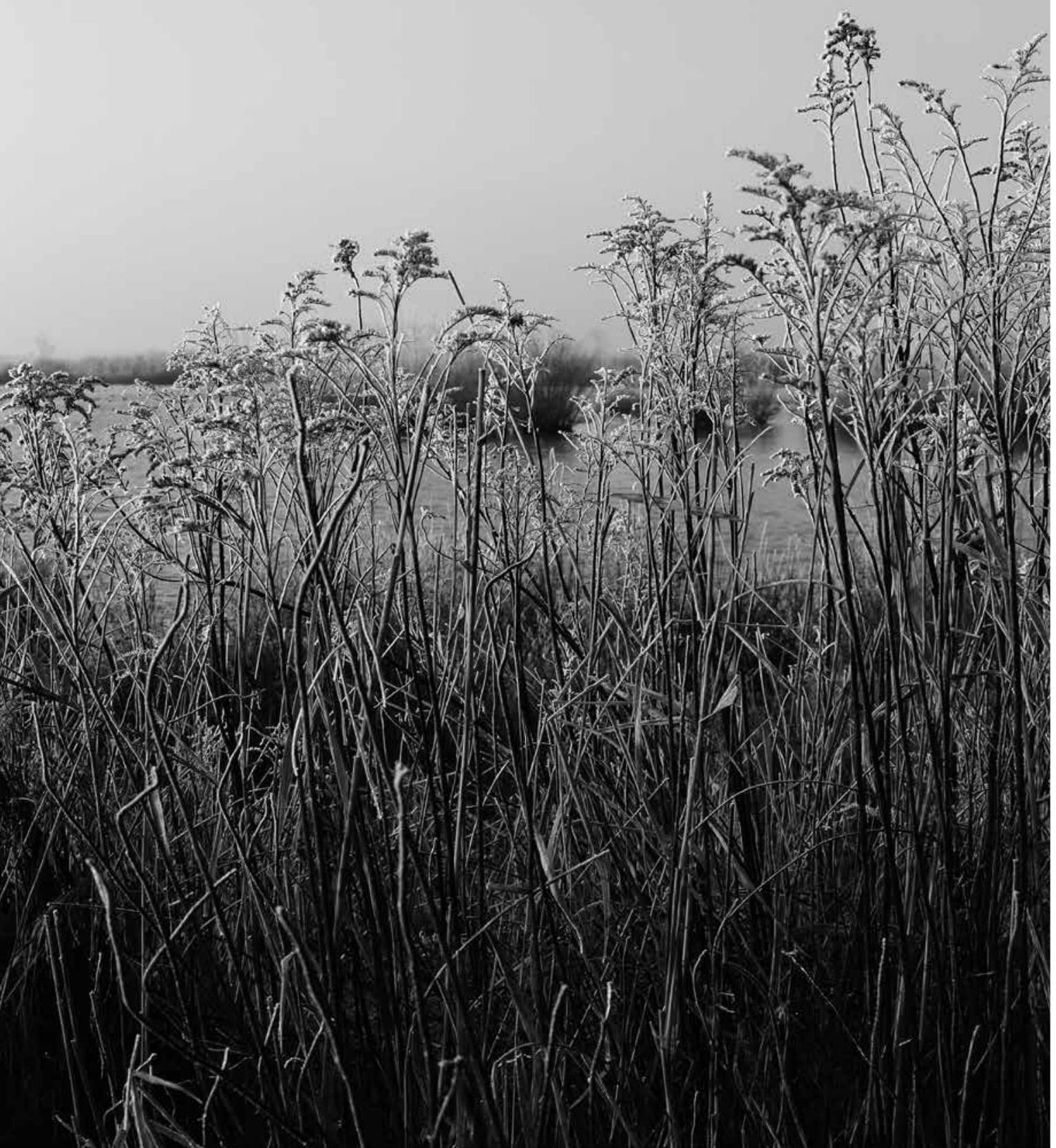

ABSTRACT - The way people or organizations describe or depict nature conveys their view of nature. In the Dutch discourse, views of nature are mostly conceived as socio-cultural constructs regarding the character, value, and appreciation of nature. Views of nature tell us how we perceive nature and how we want to relate to it. Likewise, they shape our preferences for certain types of nature. Views of nature function as frames and provide an interpretive and reflective context for our experiences with nature. Views of nature are also digestions of meaningful experiences. It is through communication that we become aware of such experiences. We argue that meaningful experiences have religious depth. On the basis of this argument any discourse on nature contains, in a sense, religious subtexts. We examine the Dutch discourse on nature.

\section{INTRODUCTION}

At the end of the twentieth century the Dutch government came to the conclusion that Dutch nature had limited ecological sustainability as a result of substantial habitat fragmentation. The Dutch Nature Policy Plan of 1990 (Ministry of Agriculture, Nature and Fisheries [Ministerie van LNV], 1990) aimed to compensate for this lack of connectivity by creating a coherent network of nature reserves throughout the Netherlands, the so-called National Ecological Network (cf. $\ 1.1$ ). With the National Ecological Network, the emphasis shifted from the conservation of 'old' nature to the development of 'new' nature, that is, from a defensive to an offensive strategy: a concept known as 'nature development'. Nature development involves technical interventions, often with respect to areas of land that were formerly used for agriculture, in order to create suitable habitat conditions, after which natural processes are given free rein. In short, nature development refers to rewilding through human intervention. Even though nature 
development was not the only goal of this former nature policy plan, it can be considered as one of the most remarkable and far-reaching objectives according to Zwanikken (2001); not the least because the development of nature is not entirely uncontroversial. ${ }^{58}$ In the Netherlands there is an ongoing battle with regard to nature, 'often fought in grimness and bitterness' (Keulartz, 2000: 75).59 This controversy indicates that 'nature' is a concept that arises in relation to 'social concerns and ideological agendas' (McGrath, 2001: 82) or 'foundational beliefs and values' (Ross-Bryant, 2013: 4).

In this study we seek to gain insight in the complicated and unique Dutch discourse on nature..$^{60}$ In the first part of the chapter we provide a socio-historical overview of Dutch nature policy, and we discuss the particular way nature has been constructed, experienced, and managed in the Netherlands. Subsequently, in the second part of this chapter we are interested in the question as to whether in the discourse about nature in the Netherlands religious elements can be identified.

\subsection{SOCIO-HISTORICAL CONTEXT OF} THE DUTCH NATURE POLICY

It was only in the early twentieth century that views on nature and conservation became part of the public discourse and policy-making in the Netherlands (Zwanikken, 2001). The period up to 1990 was marked by an increase in focus on conservation and the implementation of defensive nature policies: the objective being to retain existing nature reserves and resources. Despite attempts to preserve what was already established, circa 1990 it was concluded that a lot of ground had been lost and that nature had drawn the shortest straw (Kockelkoren, 2000). Table 1 shows the phased progression of nature policy in the Netherlands until the 1990s.
58 Zwanikken (2001) mentioned three themes that dominate the discourse surrounding the National Ecological Network: (1) varying views of nature referred to in the general public debate, (2) the debate regarding the segregation or synerg of nature with other functions, and (3) the question of whether an ecological network throughout the Netherlands is the correct instrument for shaping nature conservation policy. 59 Drenthen (2003) interestingly argued that the discussions are no about whether nature is desirable, but rather about the question as to whether the 'nature that actually exists in the Netherlands (can be) called "really" natural' combined with the question as to 'whether naturalness is actually feasible within the Dutch context' (11).

60 Here we follow Hajer (1995) in his description of discourse: an ensemble of ideas and concepts that are 'produced, reproduced and transformed in a particular set of practices' (44)
TABLE 1. Phases in the emergence of Dutch nature policy

\begin{tabular}{|l|l|l|}
\hline Period & Qualification & Description \\
\hline Pre-1920 & Developing & $\begin{array}{l}\text { The popularization of knowledge of the natu- } \\
\text { ral world resulted in an increase in interest in } \\
\text { nature and conservation. The first botanical } \\
\text { society was founded in 1845. The first books } \\
\text { on birds and flowers and plants soon followed. } \\
\text { Public interest was further boosted through the } \\
\text { efforts of E. Heijmans and Jac. P. Thijsse, who } \\
\text { first published the journal The Living Nature } \\
\text { (in Dutch: De Levende Natuur) with J. Jaspers } \\
\text { Jr. in 1896. The State Forestry Commission (in } \\
\text { Dutch: Staatsbosbeheer) was founded in 1899 to } \\
\text { manage state nature reserves and oversee wood } \\
\text { production. In 1901 the Dutch Natural History } \\
\text { Association (in Dutch: Nederlandse Natuurhis- } \\
\text { torische Vereniging) was founded with nature } \\
\text { conservation as an aim. The first key battle for } \\
\text { nature conservation took place in 1904 when the } \\
\text { city of Amsterdam moved to dump its refuse in } \\
\text { the waters of the Naardermeer. This provoked } \\
\text { a backlash in the newspaper Algemeen Han- } \\
\text { delsblad (Thijse) and in the magazine Groene } \\
\text { Amsterdammer (Heijmans), resulting in funds } \\
\text { being raised to purchase the Naardermeer. The } \\
\text { following year marked the founding of the Asso- } \\
\text { ciation for Preservation of Natural Monuments in } \\
\text { the Netherlands (in Dutch: Vereniging tot Behoud } \\
\text { van Natuurmonumenten) and the acquisition of } \\
\text { the Naardermeer. }\end{array}$ \\
\hline Expanding & $\begin{array}{l}\text { In 1929 nature conservation became a key task } \\
\text { of the State Forestry Commission (in Dutch: } \\
\text { Staatsbosbeheer). The so-called Provincial } \\
\text { Landscape Commissions (in Dutch: Provinciale } \\
\text { Landschappen) were founded soon after with } \\
\text { the objective of setting up and managing nature } \\
\text { reserves at a provincial level. Conservation orga- } \\
\text { nizations also began exercising political pressure, } \\
\text { resulting in government policy, e.g., the Forestry } \\
\text { Law (in Dutch: Boswet) of 1922 and the Nature } \\
\text { Law (in Dutch: Natuurschoonwet) of 1928. }\end{array}$ \\
\hline
\end{tabular}




\begin{tabular}{|l|l|l|}
\hline 1932-45 & Testing & $\begin{array}{l}\text { Hard times due to the economic crisis and World } \\
\text { War II. Employment became the top priority, } \\
\text { resulting in large-scale exploitation of natural } \\
\text { resources and reparcelling of land. }\end{array}$ \\
\hline 1945-60 & Defending & $\begin{array}{l}\text { Post-war reconstruction, population increase, } \\
\text { and growth in prosperity all posed a threat to } \\
\text { nature: urban expansion, road works, indus- } \\
\text { trialization, increased intensive farming, etc., } \\
\text { increasingly affected natural areas. }\end{array}$ \\
\hline 1961-69 & Recognition & $\begin{array}{l}\text { Detrimental effects of prior developments result- } \\
\text { ed in a breakthrough in awareness. Nature con- } \\
\text { servation was given recognition by policymakers } \\
\text { and the general public. Demand for outdoor } \\
\text { recreation increased, natural and environmen- } \\
\text { tal education was established, and in 1967 the } \\
\text { Nature Law (in Dutch: Natuurbeschermingswet) } \\
\text { was passed. Despite this, farming expansion and } \\
\text { reparcelling of land continued. }\end{array}$ \\
\hline $1970-80$ s & Reflection & $\begin{array}{l}\text { The economic downturn of the 1970s and '80s } \\
\text { resulted in limited government spending; the } \\
\text { budget for nature conservation was insufficient } \\
\text { to halt further deterioration of nature reserves. } \\
\text { At the same time a broader reflection had been } \\
\text { taking place on the future of nature policy. A } \\
\text { debate emerged regarding the segregation or } \\
\text { synergy of functions, particularly between nature } \\
\text { and farming. Twenty-four national parks were } \\
\text { established. }\end{array}$ \\
\hline
\end{tabular}

Source: Gorter (1986)

Zwanikken (2001) claimed that the heightened environmental awareness during the 1970s and the emergence of a unique nature reserve called the Oostvaardersplassen ${ }^{61}$ as a result of human 'abstinence' leaves room for 'the notion that nature is no obsolete, historical category, but rather a category that is always open to human opportunity' (136). A socalled 'policy window' occurred, in
61 The Oostvaardersplassen form a nature area of approximately $56 \mathrm{~km}^{2}$ located between Almere and Lelystad in the Dutch province of Flevoland. Following the reclamation of the Flevopolder in 1968 this wet land remained unused. Nature saw its chance and a marsh formed, with pools, reed beds, and willows. At the moment the area consists roughly of a wet part (approx. 3,600 ha) and a dry part (approx. 2,000 ha). The dry part is regarded as a suitable habitat for lorge her vaardersplassen are of international importance as marshland and as a wintering site for birds. In 2013 a film was made about this area entitled de Nieuwe Wildernis (The New Wilderness). which problems, policies, and politics coincided. ${ }^{62}$ The most interesting element in Dutch nature policy since the 1990s has been the concept of 'nature development' (natuurontwikkeling), which refers to creating 'new nature' (nieuwe natuur): rewilding through human intervention. As an offensive strategy, nature development has facilitated a new way of thinking about nature as well as a change in the mind-set regarding spatial planning and zoning. In a sense, nature development can be seen as a typically Dutch and inherently paradoxical concept. ${ }^{63}$

\subsubsection{The Re-emergence of Wilderness}

62 Metz (1998) argued that at

that time there was awareness of

natural and environmental issues, such as the near extinction of the otter. An equally important factor she highlighted is the breakthrough in attitudes regarding farming and agricultural policymaking: 'At the time the prediction was that the decline of agriculture would result in a surplus of some 700,000 hectares. The breakthrough in politics came at the end of the eighties under the third Lubbers cabinet (19891994), which allocated more funding for nature and the environment. At the same time, intriguing policies were being floated: ideas on the spontaneous development of nature, inspired by the living example of the Oostvaardersplassen, quickly gained popular support' (Metz, 1998:187). opment' has a mon with 'nature restoration' or 'restoration ecology': the effort to restore ecosystems that humans have turned into agro-ecosystems, human settlements, or extractive areas.

64 With regards to nature in Dutch discourse, scholars mostly speak about images of nature (natuurbeelden). To avoid 'scenic' or 'picturesque' connotations, and because our view of nature is strongly related to our world view, in this chapter we prefer 'views of nature' to 'images of nature'.
The Netherlands is said to be a human-made country; consequently, the repurposing of land for nature development was initially met with resistance. Zwanikken (2001) characterized the debate as being principally about the relationship between culture and nature and the question as to which frame of reference hould be used in regard to nature development. The choice of frame of reference for nature to be (newly) developed is connected to views of nature. ${ }^{64}$ View of nature are often categorized on the basis of the degree of human influence on nature and the question of whether or not to allow natural processes. When we look at the views of nature within Dutch nature policy, three dominant understandings stand out: the wilderness view of nature, the Arcadian view of nature, and the functional view of nature, summarized in the alliteration 'reposeful' (rustig), 'rough' (ruig), and 'rational' (rationeel) (Keulartz et al., 2002).

Van der Windt et al. (2007) pointed out that until the 1980s and '90s the nineteenth century agrarian andscape acted as a key reference point because of its high level of biodiversity and its cultural value. They further claimed that most nature organizations in the Netherlands maintained an Arcadian view of nature. Vera (2000) characterized the Arcadian view of nature as an image of a harmonious coexistence between nature and culture. The Arcadian view of nature focuses on rural nature-on nature that is characterized 
by a human-made landscape in which patterns from the past can be observed (Keulartz, 2000; Van Koppen, 2002). This perspective is idealistic and sees the pre-industrial landscape of the eighteenth-century landscape painters as a source of inspiration and retrospection (Keulartz, 2000; Lemaire, 2007 [1970]). Van Koppen (2002) spoke of a dual understanding of the concept 'Arcadia' that appreciates not only cultivated, rural nature, but also elements of wilderness, demonstrating a dialectic tension between the 'Arcadian' and the 'wilderness' view of nature. There are similarities, however, as well: the 'wilderness' and 'Arcadian' views of nature romanticize or idealize nature in a certain way ${ }^{65}$ The following quote from a public communication of Tiengemeten, a well-known nature development project in the Netherlands, is illustrative of this romanticism:

Human-sized brush and grassland, dissected by a dozen streams. Highland cattle with their legs in the water and a gap in the dike. Thousands of birds of all kinds and sizes. Chaos and silence. This is the kind of wilderness we have missed. This is Tiengemeten the primeval delta now!... It's quiet in the Wilderness. Quiet and yet bustling with life... The hill is the best vantage point to catch a panoramic view of the Wilderness, the Haringvliet with its locks and fixed embankment on either side. A safe place 'separated from the world' (Natuurmonumenten n.d.: 1,6).

Ever since the 1980s there has been a reemergence of the wilderness ideal in the Netherlands (Buijs 2009). Vera (2000) characterized the wilderness as the 'primitive image' in the philosophy of nature. According to this view, nature has an untamed and pristine character and because of that it is regarded as something primordial. It refers to uninhabited and uncultivated spaces and to an 'environmental system where natural processes occur with little or no significant influence by human beings' (Taylor, 2012:293). According to Drenthen (2003:200), the wilderness approach builds on the idea 'that wild, uncultivated nature represents an important value that is to be protected from cultivation and appropriation by humans'. In effect, wilderness is one extreme on the scale of decreasing naturalness and increasing human influence. The other extreme is the functional view of nature, which holds that nature is an object of production: a view of nature with a strongly anthropocentric character. Or as Buijs et al. (2009) suggested:

The wilderness image is based on ecocentric values of nature and a very narrow definition
65 Van den Born and De Groot (2011) concluded that the Dutch romanticize nature. Empirical re search by De Groot and van den Born (2003) showed that 'th spiritual/romantic image of participation in nature is present as an undercurrent' among the Dutch. The large majority of their respondents preferred 'wild and experiential landscapes types' (134). of the concept of nature, related to the autonomous development of nature. The functional image, on the other hand, focuses on anthropocentric values in which nature should be intensively managed, for aesthetic as well as utilitarian purposes. Within this image, nature and culture are not seen as opposites; humans are supposed to master nature (Buijs et al., $2009 \cdot 114)$

Van der Windt et al. (2007) have suggested that the re-emerging idea of wilderness, or autonomous nature, became a topic on the political agenda as a result of the efforts of the action groups in the 1980s-1990s. These ecologically minded groups demanded that attention be devoted to the repair of entire ecosystems and campaigned against the economic exploitation of forests. ${ }^{66}$ The ecological reference point started to act as a criterion for assessing the current state of nature and as a basis for the formulation of policy objectives. Here the central question is how nature would have been if it had not been exposed to culturally informed human interventions. In 1989 a policy document called Nota Nature Development linked the ecological reference point to prehistorical, primeval nature (Ministry of Agriculture, Nature and Fisheries [Ministerie van LNV], 1989). The underlying thinking was that nature functioned at its best before it was influenced by humans. The Dutch Nature Policy Plan of 1990 (Ministry of Agriculture, Nature and Fisheries [Ministerie van LNV], 1990) expanded the ecological reference point. Rather than referring to something vague, such as 'prehistoric nature', it refers to what is generally considered to be the last vestige of wilderness: primeval forest in Europe, namely the Bialowieza Forest 66 During the 1980s the Dutch (Puszcza Bialowieska) in Poland. branch of the WWF advocated the development of ecosystems that were to remain more or less free From the above discussion we may conclude that the from human influence because of concept of 'nature development' with the emphasis the intrinsic value of nature or for
reasons of extensive recreation. on 'primeval nature' or 'autonomous nature' was priVan der Windt et al. (2007) stated that there were efforts to realize hese ecosystems along the rivers, as it was expected that here the greatest natural diversity could develop. Furthermore, they pointed to the fact that from the 1990 s on more traditional NGOs also became interested in wilderness. Van der Windt et al. (2007) argued that hese developments were reflected

in government policy, as evidenced The concept of nature development is linked to that of by the relevant policy documents. marily an ecological frame. The question arises as to how nature development came to resonate so strongly within society that it could dramatically shape Dutch nature policy. To answer this, we will explore the 'new nature paradox', as we call it.

\subsubsection{The New Nature Paradox}


thing Schouten (2002) considered to be a novelty in history. He also foregrounded other notions about primary nature:

The final decade of the twentieth century, in which the boundary between urban and rural largely disappeared and Arcadia came to resemble an agrarian industrial park, saw the rebirth of the myth of virgin wilderness. And of that of the noble savage, who was elevated to a conservationist avant la lettre... In the past primeval wilderness was largely seen as dense forest. Now, certain ecologists depict it as an open woodland landscape in which herds of large herbivore roam. In addition, the swamp, which was previously seldom ascribed a positive role in the wilderness myth, now gains an aura of venerable age (Schouten, 2002:33). ${ }^{67}$

Zwart (2002) called for a radical shift in attitudes: one that respects 'exalted primeval nature' and criticizes the 'view of nature of the [Dutch] Golden Age (seventeenth century) and that of modern engineers' (46). This shift is a recurrent topic in the discourse on nature, sometimes referred to as 'new thinking', exemplified in the following quote:

Tiengemeten: new nature, breaking ground like the water of its creeks. The symbol of new thinking, of trust in the shaping capacity of nature. Primeval nature, nature that seems to have been ever-present (Natuurmonumenten n.d.: 17).

Intriguingly, the new nature concept is in itself paradoxical. It links with current ideas on engineerability and technology, whilst striving to achieve a prehistorical primeval type of spontaneous nature independent from humankind. Kockelkoren (2000) regarded the fear of the encroaching technological culture - which had resulted in a reduction in natural diversity in the Netherlands over a short period of time - to be a feeding ground for the ideal of autonomous nature and the idea of nature development. In a sense, however, it is also a technical understanding of wilderness nature, the result of 'a slow-motion ballet méchanique of draglines and bulldozers, excavators and trucks' created 'under the direction of engineers and geomorphologists'
67 In this context Van der Woud (2007) wrote: 'The cultural landscape is also disappearing because propaganda is made for another, brand-new landscape image, and we are being manipulated to like that image... The liberation of nature started in 1990 with the National Ecological Network and an end was put to... a four-centuries-old national tradition of lan reclamation... We cannot deny tha this new nature has an importan ethical dimension. It gives the impression of having a prehistoric or early medieval antiquity-a landscape that existed before human began to tinker with it and began to enslave nature' $(3,10,11)$.

(Hajer, 2003:90). The above discussion illustrates that attitudes toward wilderness have changed in the Netherlands. Previously, the ideal of unspoiled wilderness was projected on or recognized in existing nature reserves that were protected from human influence. Nowadays, the desire for wilderness serves as justification for human interventions in the context of the creation of 'new' nature, of which the National Ecological Network is a good illustration (Drenthen, 2003). In this respect, Keulartz (2009a) speaks of the 're-creation' metaphor, which stresses authenticity. ${ }^{68}$

The use of technology regarding nature employed by the Dutch is by no means new. Zwart (2002) noted that land-engineering practices began around $800 \mathrm{CE}$, with dykes being built, waters drained, and land reclaimed. He argued that a more active attitude regarding nature developed from that period onward that included the cultivation of the Netherlands. He linked this process to what he regarded as the ideological shift taking place during that time: the 'christianization of the low countries' (kerstening van de lage landen). For example,

Christians regard themselves as stewards of God, charged with rehabilitating nature that has run wild and providing it again with a human dimension... Christianity provided the impetus and legitimized a more active presence of humans in the Dutch landscape. The dyke served to demarcate the Christian and non-Christian world (Zwart, 2002:40).

If, as Zwart (2011) argued, most transformations of

68 For Keulartz (2009a), authenticity had always been the guiding element within the world of conservation, but slowly there has been a shift from the conservation and protection of existing nature to the development of 'new' nature. In this respect he speaks of a shift from the 'restoration' metaphor to the 're-creation' metaphor.

69 In the past the Dutch have wrested land from the sea by turning the swampy and unstable river delta into a country with a strong agricultural identity. This process is

known as 'inpoldering'. Nowadays,

for nature-development reasons,

a lot of farmland is to be flooded again, a process called 'depoldering', which means 'giving land back to the water' the countryside are linked to Christian-conversion offensives, then conversely there may also be a correlation between 'depoldering' and secularization, ${ }^{\circ}$ between the rise of new nature and a shift in spiritual experience. Interestingly, Taylor (2012) observed that the efforts to establish and protect wilderness areas can be understood 'to be a way of establishing such places as sacred places, as temples for those who have left behind conventional religions' (310). By this he implied that 'wilderness' has acquired religious meanings.

\subsubsection{Nature Experience}

The past decade has notably seen even greater emphasis on nature experience (natuurbeleving) in Dutch nature policy. ${ }^{70}$ Kockelkoren (2000) suggested that 
near the millennium a shift took place. Buijs (2009) referred to 'a shift from an ecological focus to a combination of an ecological and societal focus' (17). This change-'to include the social values of nature'-is illustrated by the title of the second Dutch nature policy plan: 'Nature for people, people for nature' (Ministry of Agriculture, Nature and Fisheries [Ministerie van LNV], 2000). The plan works on the principle that nature should match the needs and desires of Dutch citizens. Nature should be at the heart of society (Keulartz, 2009b). Buijs (2009) suggested three changes that this plan entails: (1) a shift of focus away from ecological values to a broad range of values, including the 'experience value of nature'; (2) a broader definition of nature, which not only includes officially recognized nature reserves, but also green belts in cities and agricultural areas which may also have ecological value; and (3) a collaboration among farmers, citizens, and private companies-that is, the 'incorporation of several non-governmental actors in the implementation of nature-related policies' (18).

In 2014 the Dutch government presented its vision for the future, which shifted focus from the protection of nature from society to the strengthening of nature by society. In terms of the policy document, the focus is on 'nature that needs no protection from society, but rather is an inextricable and vital part of that society' (Ministry of Foreign Affairs [Ministerie van Economische Zaken], 2014: 7). The document claims that the distinction between 'true' nature and 'other' nature is outdated. Furthermore, it refers to 'human self-interest', given that citizens require nature to 'recuperate', seek 'excitement and wonder' in nature $(6,14)$, and wish not only to protect nature but also 'to experience and make use' of it (16). Nature development is no longer only driven by the ideal to restore 'pure nature' for the sake of nature herself, but also by the value which such nature has for the human experience.

Buijs (2009) flagged an increase in what he called 'post-material values', with urbanization and a fast-paced daily existence resulting in an increase in nature-related leisure activities. He additionally referred to a 'consumption of natural areas' (19) that is primarily symbolic, as people mainly pay attention to the symbolic meaning of a natural area: 'Traditional rural areas remind us of bygone days, while the emergence of new life in spring reminds
70 Buijs (2009) pointed to individual needs, motivations, experiences, and behaviour becoming more and more diverse. This was illustrated by a study $(43,365$ respondents) carried out by Natuurmonumenten at the end of 2014 participants preferred a persona experience of nature; tranquility, purity, and natural beauty are cited as key aspects when visiting nature. The use of language in the study is intriguing. Reference is made to a 'nature experience agenda' and an 'experience package'. Another interesting aspect is the approac of Natuurmonumenten that 'nature conservation starts with experiencing nature', because 'an environment we [feel] connected to.... is worth cherishing' (2014:9). us of the spiritual or divine basis of human life, and the decay in autumn reminds us of our mortality' (19).

McGrath (2001) interestingly argued that in Western thought the term 'nature' has the connotation of innocence and nostalgia-the memory of a distant rural past in which 'the simplicity and beauty of nature is contrasted with the artificiality and ugliness of human conventions and creations' (82-83). In other words, he spoke of the romanticization of nature. Romanticism in particular has been crucial to our current way of thinking about wilderness and is at the root of the current nature and environmental movements, according to authors such as Nash ([1969] 2001) and Cronon (1995). However, romanticism is difficult to define. In general, it implies an enthusiasm for the strange, the distant, the lonely, and the mysterious (Nash, [1969] 2001). Romanticism consequently has a preference for wildness and for the primitive, as Nash ([1969] 2001)) suggested: ${ }^{7}$

Primitivists believed that human's happiness and wellbeing decreased in direct proportion to his degree of civilization. They idealized either contemporary cultures nearer to savagery or a previous age in which they believed all men led a simpler and better existence (Nash, [1969] 2001: 44, 47).

Along this line of thought, Ester (2012) wrote that one of the benefits of Romanticism was 'that nature was no longer seen as a self-sustaining mechanism, but as an animate organism that appeals to the feelings of humans' (12). The appreciation of nature in our present century and 'the fear that vital nature values will perish through rampant consumerism can be traced back to the profound feeling of the Romanticists' (12). He therefore stressed that Romanticism had a strong religious orientation, and that the emphasis on emotion was not so much a sentimental impulse but an expression of trust in the truth of feeling. True feeling is 'a pure response to the appeal that the divine generates in 71 Nash ([1969] 2001) pointed humans' (12). Such Romanticist feelings are a source out that this has had far-reach- of religion, however much 'The sentimental, romantic ing consequences for our thinking attitude with which "romantic" is associated nowadays the sublime and picturesque led the is a degeneration of the original profundity of the way by enlisting aesthetics on wil- romantic sense of life' (13). derness's behalf while deism linked nature and religion. Combined with the primitivistic idealization of a life closer to nature, these ideas fed the Romantic movement, which had far-reaching implications for It is impossible to discuss nature in a timeless way and wilderness' (44). 
od people talk about 'nature', but always in a different way (Roothaan, 2005). ${ }^{72}$ Views of nature are therefore historically situated; Schouten (2005), for example, considered views of nature as cultural phenomena: 'In the way nature constantly brings forth new life and new forms, culture, too, constantly creates new views of nature. In doing so, the variation in perceptions, ideas and views seems hardly inferior to the richness of forms in nature itself' (9).

The multiformity of nature-the 'many faces of nature', as McGrath (2001) and Van Koppen (2002) significantly put it-is a reflection of the diversity of lifestyles and life views. Different social interests and different individual lifestyles, wishes, and desires are projected onto nature (Drenthen, 2003). McGrath (2001) argued that the way in which nature is conceptualized in various cultures provides insights both into these cultures and into the concepts themselves. Accordingly, the 'instability' of the concept of nature illustrates why nature has no fixed references but is defined by communities of discourse (103). In other words, we need communication to gain an understanding of nature. But each description or conception of nature reflects 'a complex amalgam of religious beliefs, popular sentiment and the vestiges of a classical culture' (103). The qualification of something as 'nature' always implies a particular view of nature. Such a perspective does not so much provide information about nature as shows how we perceive ourselves in relation to nature (Schouten, 2005). Or as Ross-Bryant (2013) argued, 'Nature... grows out of our world view and shapes our ways of acting in the world' (4). In other words, views of nature can be seen as articulations of our worldview. ${ }^{73}$ In the following, we will further elaborate on this aspect by approaching views of nature as frames.

\subsubsection{Views of Nature as Frame}

Keulartz et al. (2004) considered views of nature as socio-cultural constructs regarding the character (cognitive dimension), value (normative dimen-
72 Roothaan (2005) distinguished in this regard three main periods in Western history Starting with the pre-modern period (which centered on the experience of the divine order; god is present in nature or the cosmos is created by God), we have moved through the God, we have moved through the functioned according to mathematical laws and was considered to be a neutral object of study) into the postmodern period (in which nature is not neutral but has its own more or less constructed intrinsic value).

73 For Naugle (2002) 'world views are undoubtedly contextual phenomena enabling people to see things and make connections' (150). Worldviews define the person and provide humans with 'fundamental assumptions upon which a life is based' (291). Naugle (2002) said that a worldview is the prima ry system of narrative signs that articulate a vision of reality and lie at the base of individual and collec tive life [and] is the most significant set of presuppositions on the basis of which interpretation operates (313). He came to the conclusio that any interpretation of the socia and natural world is conditioned by worldviews, including our interpretation of and relation to nature. sion), and appreciation (expressive dimension) of nature. The cognitive dimension pertains to knowledge of nature. The normative dimension refers to our relationship with nature and the moral status we ascribe to nature. It also pertains to the ethical criteria regarding our relationship with nature. Finally, the expressive dimension concerns the way we experience nature aesthetically and emotionally. Table 2 connects the three major views of nature in Dutch nature policy with the cognitive (ecological theory), normative (ethical perspective), and expressive (aesthetic perspective) dimensions.

TABLE 2. Views of nature classified according to their cognitive normative, and expressive dimensions.

\begin{tabular}{|l|l|l|l|}
\hline & Ecological Theory & Ethical Perspective & Aesthetic Perspective \\
\hline Wild nature & Systems ecology & Ecocentric & Objectivist \\
\hline Arcadian nature & Community ecology & Steward/Partner & Subjectivist \\
\hline Functional nature & Production ecology & Anthropocentric & Formalist \\
\hline
\end{tabular}

Source: Keulartz et al. (2004)

74 Views of nature have a clear relationship with landscape preferences. However, 'an important difference between images of nature and landscape preferences is that images of nature are cognitions about nature (e.g. general values $p$ and beliefs). Landscape preferences are usually conceived of as predominantly based on precognitive, affective responses to the physical envirc feelings of liking or disliking. They are often defined as the aesthetic or evaluative response elicited

by visual encounters with real or Frames lead people's thoughts and discourses by presimulated natural settings' (Buijs et 2009:114). Furth is et al. (2009) noted that 'images of nature have significant power to predict preferences for non-urban landscapes. People with a functionor an inclusive image of nature showed lower for natural landscapes, while people with a wilderness image showed a higher relative preference for natural landscapes' (121).
With the above points in mind, we conclude that views of nature tell us how we perceive and want to relate to it. Such views similarly organize our preferences for certain types of nature. ${ }^{74}$ Therefore, the literature justly indicates views of nature as frames of reference on the basis of which preferences of nature are formed, and as interpretive frameworks by which xperiences with nature gain meaning (Keulartz et al., 2002; Buijs et al., 2009). senting the world in a particular way and attributing meaning to human experience. In other words, it is through frames that people are able to make sense of the world, and experiences become meaningful. We frame nature through visual representations as well as hrough the language we use to describe it (figure 1). Such framing entails an interplay between surface frames and deep frames (e.g., Lakoff 2006). 
FIGURE 1

Interplay between frames

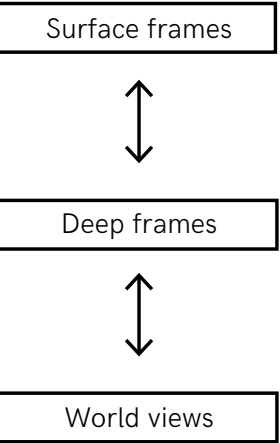

Surface frames function at the level of our daily language. For example, the words 'dark forest' first of all have a descriptive meaning for a certain type of woodland and 'wilderness' refers to a rugged place. Through their immediate meaning, surface frames identify the context of the discourse. However, these surface frames appeal to underlying values and convictions that can be communicated in deep frames and ground our daily language in our normative convictions regarding the world and our lives.

Deep frames articulate our worldviews and hence are more fundamental than surface frames. The frame 'dark forest' is, for instance, associated with feelings of fascination, fear, initiation into a numinous reality, and so forth. Moreover, the frame 'wilderness' has (in the present discourse on nature in the Netherlands) mostly the connotation of pristine and 'real' nature-nature as it originally was before it was affected by humans. Deep frames provide the background we need to interpret something as meaningful. In doing so deep frames provide an interpretive context for our experiences of nature.

\subsubsection{Meaningful Experiences}

Experiencing nature as meaningful can influence one's view of it. Buijs et al. (2009) spoke of views of nature as 'cognitive reflections of prior experiences with discourses about nature' (144). Drenthen (2002:78) likewise suggested that our different interpretations of nature are responses to appealing experiences in nature. For Drenthen (2002), nature presents a pretense of meaning, something that captures our attention, however unconsciously. For this reason, we argue that meaningful experiences precede views of nature, which are (theoretical) interpretations of experiences that we acquire in nature. ${ }^{75}$ At the same time, experiences often acquire meaning from people's views of nature. A circular relationship therefore exists between meaningful experiences and views of nature (figure 2).

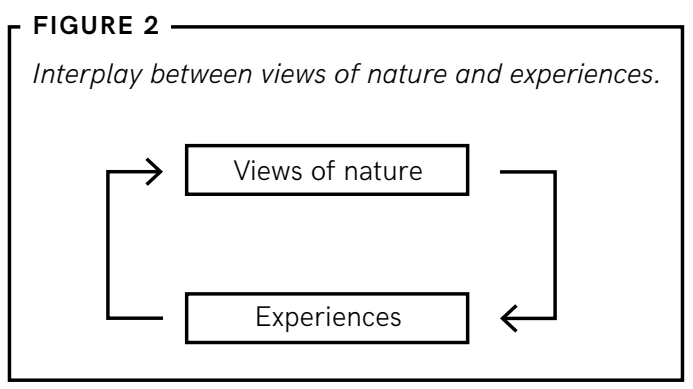

Experiences are often personal and depend on the location. ${ }^{76}$ This makes them difficult to define and examine. Often they cannot be reproduced and are not easily verified. Because experiences are difficult to measure, they must be disclosed through communication. Although the meaning lies in the experience itself, the meaning may only become explicit when it is verbally expressed. When our experiences of nature and our life stories are dialogically 75 This involves some form of connected, nature acquires deeper meaning (Dreninteraction: when dealing with such then, 2011). It is in communication with others that experiences we are directed by our we articulate our experiences and integrate them into our worldviews are influenced by the stories of our lives. To wit: our experiences, emphasizing the relationship between views of nature and worldviews.

People always tell stories about [themselves] to an to bes sonal, it remains to can speak of purely individual experiences. Some experiences are enhanced by the feeling that they re shared, such as watching a football game together. There are also certain emotions that some can only experience in solidarity with others, such as feelings of comfort and security. What may be considered as an individual experience at first sight is strongly influenced or enhanced by socio-cultural elements (Taylor, 2003). Experiences may seem available separately, but they should not be considered independently. mbedded in a relationship and in order to render heir storied-selves intelligible, people must draw on harrative resources that circulate more broadly withsociety-embedded in a social context... People do not tell stories in a vacuum. They learn what types of plots can be told in particular contexts by being exosed to other stories. The plotlines available in any culture or institutional context can be understood as arrative resources (Willis, 2011:93, 101).

In this passage Willis (2011) shows that to be processed, experiences need a supporting narrative-in 
terms of this chapter, a deep frame-not only to interpret experiences but also to place them within the socio-cultural tradition to which one belongs. Apart from orientation, the supporting narrative (= deep frame) offers direction because it contains assumptions about how reality is or ought to be (= worldview). ${ }^{77}$ Taylor (1991) argued, for example, that humans are not simply autonomous individuals but have always been part of a cultural tradition that provides orientation and direction in life and is not only cognitive, but also evaluative. ${ }^{78}$ In addition, we derive our expressions and our words from tradition. Language is not mine alone; it was not invented by me, and it shapes or frames me. As Taylor (2003) put it:

Experiences require some vocabulary, and these are inevitably

in large part handed to us in the first place by our society, whatever transformations we may ring on them later. The ideas, the understanding with which we live our lives, shape directly what we could call religious experience; and these languages, these vocabularies, are never those simply of an individual (Taylor, 2003:40).

Here Taylor (2003) suggested that meaningful experiences in nature are felt collectively and often have a spiritual or religious depth dimension. Or as Champ (2009) noted, nature is a source of 'deeply meaningful experiences one might call religious or spiritual' (226)..$^{79}$

\subsubsection{Religious Depth Dimension ${ }^{80}$}

Several authors have noted that there is a religious revival going on in what Stolow (2005) called 'secular
77 Some experiences can occur only if one has embraced a specific narrative. The same applies to the relationship between experiences and deep frames. These are dynamically involved with each other: they influence and correct each other. This is certainly true when we talk about experiences in as deep frames may function as a meaningful framework to interpret these experiences. On the other hand, a deep frame can shape certain experiences.

78 As human beings we need evaluative or mora horizons, or to quote Taylor (1989), ' 'frameworks of understanding', in order to relate to the world in which we are living (26). We need a shared moral space to distinguish between good and evil, on matters that are or are not worthwhile, interesting or trivial.

79 On this topic Champ (2009) wrote, 'We are re-examining the way we have imagined meaning to happen in culture, opening the door for new possibilities, such as the realization that the influence of institutional religion is often important, but not always the only meaningful source in the public sphere' (237). Based on some other authors, Ross-Bryant (2013) spoke about three 'symbolic centers which have oriented people' in Wester history: 'God, humanity and nature' (3). The process of orienting she denoted as religion: 'We can describe religion as the process of orienting self in community and world, establishing through negotiation how a community lives together in light of a larger purpose or meaning' (3-4). Taylor (2001) remarked that the term 'spirituality' is nowaday replacing the word 'religion' more and more when referring to that which affects us at the deepest levl. He pointed out that 'this usage has drawn the increasing attention of scholars, who seek to defin the various meanings of spirituality and understan the perceptions and experiences that have led to the increasing popularity of this term' (175). modernity'. We live in a time that, to some, what was supposed to be secular has become sacred and what was traditionally associated with the sacred has been secularized (Hoover, 1997). In the wake of modernity, religion did not disappear, but took a different shape and form: this 'new visibility of religion should not be taken to signal something entirely new, but rather to potentially reveal previously disguised aspects of religion' (Meyer, 2012: 6). ${ }^{81}$

Authors including Hoover $(2002,2006)$ and Winkelaar (2005) have concluded that religion must not only be interpreted in terms of institutions, doctrines, and structures. Hoover (1997) characterized current religiosity as 'doing' instead of 'belonging'. He observed that at the center of this

80 It is not the intention of this 'doing' type of religiosity is the human as a seeking, chapter to comment on what religion substantially is or is not. Rathr, we seek ways to Voye (2004) called implicit religion, which 'refers to those aspects of
ordinary life which seem to conordinary life which seem to conwhe ditionally described as "religious"' (202). Ross-Bryant (2013) spoke of 'religion of everyday life' or 'lived religion' and observed that this 'will always be situated or contextualized, will always be dynamic, and will never be without ambiguities' (4) 81 In this context, Meyer's (2012) approach is interesting. She argued for a so-called 'post-secular' perspective 'that no longer takes secularization as the standard intrinsic to modernity, being alert instead to the specific ways in which the concept, role and place of religionand its study-have been redefined with the rise of secularity' (6).

82 Hoover (1997) argued that traditional movements are becoming a smaller factor in contemporary religious practice and that 'in contemporary life, the ways of being religious have moved out of the protected sphere of religious institution and tradition, and into the open ground of the symbolic marketplace' (4).

\section{questing, autonomous self. ${ }^{82}$ According to Hoover} (2002), the present forms of religion focus more on the expressed and the experienced than on the ascribed. Along this line of thought, Stolow (2005) commented, It seems no longer possible to contain religion within the confines of "traditional" social logics of institutional loyalty, the performative demands of face-to-face interaction, the controlled circulation of sacred texts, or the localized boundaries of "ritual time"” (122-123).

As he examined the roots of religion in the context of contemporary understandings, Taylor (2010) came to the conclusion that religion has to do with that which connects and binds people to what they value most and consider or experience as sacred (see alsoTaylor 2001, 2004). An example of this can be found in this statement in Roots Magazine:

Michelangelo's portrayal of The Creation of Adam, of God and Adam reaching out to one another on the ceiling of the Sistine Chapel is famous the world over. Moments like that-of reaching out and finding one another-are the best. Very rarely will you ave such an experience in nature. Practically every nature lover will have a vivid recollection of having ecome one with nature. Call it magical, call it divine Roots Magazine, 2013, Issue 10: 3 ) 
Authors such as Hoover $(2002,2006)$ and Winkelaar $(2005)$ argued that organized forms of religion no longer have a monopoly on religious experiences. Van Saane (2002) held that religious experiences occur in situations in which humans are confronted with something or someone completely different that transcends everyday life and the perceptible reality. Alternately said, 'In the encounter with humans, nature becomes a source of imagination and can convey non-objective perceptible realities' (Waaijman, 1994: 20). What is real and visible evokes thoughts and feelings 'which are not associated as much with that visible reality but rather with what is referred to' (Van Saane, 2002: 49). The visible becomes a symbol, the visible refers to the invisible (Van Saane, 2002). In this sense, religion becomes the practice of making the invisible visible through 'multiple media for materializing the sacred' (Orsi in Meyer, 2012: 24), a bridge to make the absent visible, a possibility to connect 'there' to 'here'. ${ }^{83}$ Religion provides the content or 'material'- for example, words, symbols, and rites-to interpret meaningful experiences in nature.

\subsubsection{Religious Subtexts}

The question arises as to what exactly these religious elements are and how they resonate in the discourse about nature, viz. communication of NGOs as well as in Dutch government policy on nature. The first salient fact is that both policy and the broader discourse appeal to an escape from daily life, as illustrated below:

Everyone needs to get away from the grind of everyday life, don't they? So why not take a trip and discover an island of tranquility, space and distant horizons (Natuurmonumenten, 2012: n.p.)

Looking to enjoy the tranquility of the starry night sky? Then the island of Tiengemeten is the perfect place for you. Nature has been allowed to run its free course over the last few years. Tranquility and open spaces characterize the island, leaving the bustle of the Randstad far away.

Tiengemeten is a popular location for day trippers looking for a relaxing day out. But you can only really unwind once on a lengthier stay... Leave behind the fast-track life of the city and come to rest. Spend the night on an uninhabited island, watch the sun rise over the Haringvliet, take a twilight walk while the birds
83 Meyer (2012) approaches religion as a mediation practic through which a reality is created that is perceived and experienced as real. Her emphasis on mediatio practice implies the need for what she calls material forms that can bring about this reality. For Meyer(2012) the senses are the most religious instruments by far. float down to search for a nesting place. Take in the night sky as you've seldom seen it (VVV Zuid Holland Zuid, 2012).

Often some sort of promise is made: recharge, find yourself, purify, come back reborn, a new world, and such:

The nature island in the Haringvliet is the place to recharge... The ferry voyage already gives you a sense of shedding the pressure of everyday life. And once you disembark on Tiengemeten, you're setting foot on a new world (Natuurmonumenten, 2011: 20)

A network to end the fragmentation of nature. Its goal being to repair biodiversity and to create a stunning green environment for recreation, relaxation, a place to retreat from the humdrum of daily life in the urban jungle and find ourselves, to recharge and get on with it. Literally, to recreate (Nationaal GroenFonds, 2010: 7).

In short, nature is referred to as being 'the better world', in which it becomes a sort of symbol for Paradise, with silence presented as an instrument for spiritual experience. ${ }^{84}$ Such sentiment is exemplified in the following passages:

Where in today's world is a place where the horizon stretches further than the eye, where there are no footpaths, and visitors can ponder amidst the birds? Where silence is audible, open space is tangible and you can come to rest? Where the waters are wild and untamed...feeding and drowning?... The world is miles away, worries melt and a smile breaks through (billboard, visitor center, Tiengemeten, Natuurmonumenten).

Nature is the perfect place for people to unwind... It offers the necessary space to find yourself in silence. Not only during the day, but at night when the flickering stars in the night sky add an extra dimension of rest and silence around you (Ministry of Agriculture, Nature and Fisheries [Ministerie van LNV], 2000: 9).

to fill a vacancy with a communi- Waaijman (2000) pointed out that religious experienccation professional who is able to es do not have to be groundbreaking or transcendent bring the experience of the attention the attention of the public-one of the ideas is to promote Tiengemeexperiences. They can also include experiences that affect human existence, serving as ethical encounters en as an icon of silence. 
fear, truth, and/or beauty. Willis (2011) noted that wilderness has been rediscovered as the place of the sublime, which is often associated with an experience of nature that is without comparison, grand, and overwhelming-an experience that goes beyond our sensual or intellectual capacities. This statement demonstrates how such perception can be reflected in public communication:

The Oostvaardersplassen. Open spaces stretching from horizon to horizon. A dynamic open space where all is connected. Marshes seamlessly meld into untouched, pristine reed banks. Nature is allowed to run its course, following the rhythm of the seasons and the circle of life. Over and over again. The inhabitants of the Oostvaardersplassen don't give it a second's thought. They hunt, fly, feed, rest and mate. It is eat or be eaten. It is life as it should be. In the here and now-no more, no less. Where earth and heaven intersect (billboard, visitor center, 'De Oostvaarders', Staatsbosbeheer).

In a sublime experience we transcend our sensory world and have an experience that exceeds us. We feel we are part of a reality that is larger than us, in which we experience how small we are, and at the same time we feel connection. The following show how this is reflected in discourse about nature in the Netherlands:

We believe that it is crucial that people foster a strong connection with nature... Make friends with animals, trees, plants, the sun and the stars. And discover who you truly are: one of Mother Nature's children (advertisement of Staatsbosbeheer, 2014).

I'm in that landscape, I'm part of it. Unio mystica. Connection and total detachment at the same time. Nothing can happen to me (Sinke, dir., 2010).
He refers to this as 'deism'. Nash ([1969] 2001) noted that since the beginning of thought men have believed that natural objects and processes had 'spiritual significance', 'but "natural" evidence was usually secondary and supplementat to revelation. And wilderness, somewhat illogically, was excluded from the category of nature. The deists, however, based their entire faith in the existence of God on the application of reaso to nature. Moreover, they accorded wilderness, as pure nature, special importance as the clearest medium through which God showed His power and excellency' ([1969] 2001 46). Later he remarked that 'by the mid-eighteenth century wilderness was associated with the beauty and godliness that previously had defined it by their absence. People found it increasingly possible to praise, even to worship, what they had formerly detested' (2001 [1969]: 46). He also pointed out that in the course of time people began to perceive religious elements in nature itself. 'In the sweep of Western thought, he argued, 'this was a relatively young idea, and one with revolutionary implications. If religion was identified with wilderness rather than opposed to it, as had traditionally been the case, the basis for appreciation, rather than hatred, was created' (Nash, [1969] 2001 56). 'Romanticism, including deism and the aesthetics of the wild', he concluded later on, 'had cleared away enough of the old assumptions to permit toward wilderness without entirely eliminating the instinctive fear an hostility a wilderness condition had produced' ([1969] 2001: 66).
Nash ([1969] 2001) intriguingly argued that sublimity implies a connection between God and the wilderness. ${ }^{85}$ Willis (2011) additionally noted that through a sublime experience, 'certain landscapes came to be valued as holding the promise of revealing the face of God' (95). Conversely, we found no mention of (a personal) God in our study sample.

\subsection{DISCUSSION}

In the Netherlands the concept of nature development has facilitated a new way of thinking about nature. Whereas the wilderness ideal previously led to the protection and conservation of endangered, pristine environments, today it serves as a justification for human interventions in the creation of 'new nature'. Nature development in itself, therefore, is paradoxical: it links with current ideas on technology, whilst striving to achieve a prehistorical type of nature independent from humankind. This means that a technical understanding of wilderness is central to the Dutch discourse of nature. In one sense, the technical attitude toward nature is still alive in the Netherlands through depoldering, which transforms farmland into wilderness. Nevertheless, there is an increased sense of loss of contact with nature, which has resulted in calls for 'more real nature', or wilderness. In the Netherlands, strictly speaking, there is no true wilderness remaining. Hence, the Dutch have attributed values such as 'real' or 'pure' to cultural landscapes in order to find any 'wilderness nature' in their country and have sought to create new wildernesses through nature development.

Awareness of the interplay between surface frames and deep frames helps us to value more the discourse about nature for several reasons. First of all, we learn how communicating about nature is connected to worldviews. For, our views of nature convey more about how we perceive ourselves in relation to the natura reality than about the reality we call 'nature'. Further, we come to understand how contact with nature may lead to meaningful experiences. It is through communication (frames) that experiences become meaningful. Or more specifically, deep frames provide an interpretive context for our experiences with nature. This study has hopefully shown that the discourse in the Netherlands on nature, as found in governmental and NGO produced communication about nature, contains religious subtexts that are not necessarily theological. Our examples suggest, rather, that nature is valorized and 'religionized' without any mention of a personal god or other supernatural agents. That might be part of the success of this discourse in a decreasingly Christian country. 

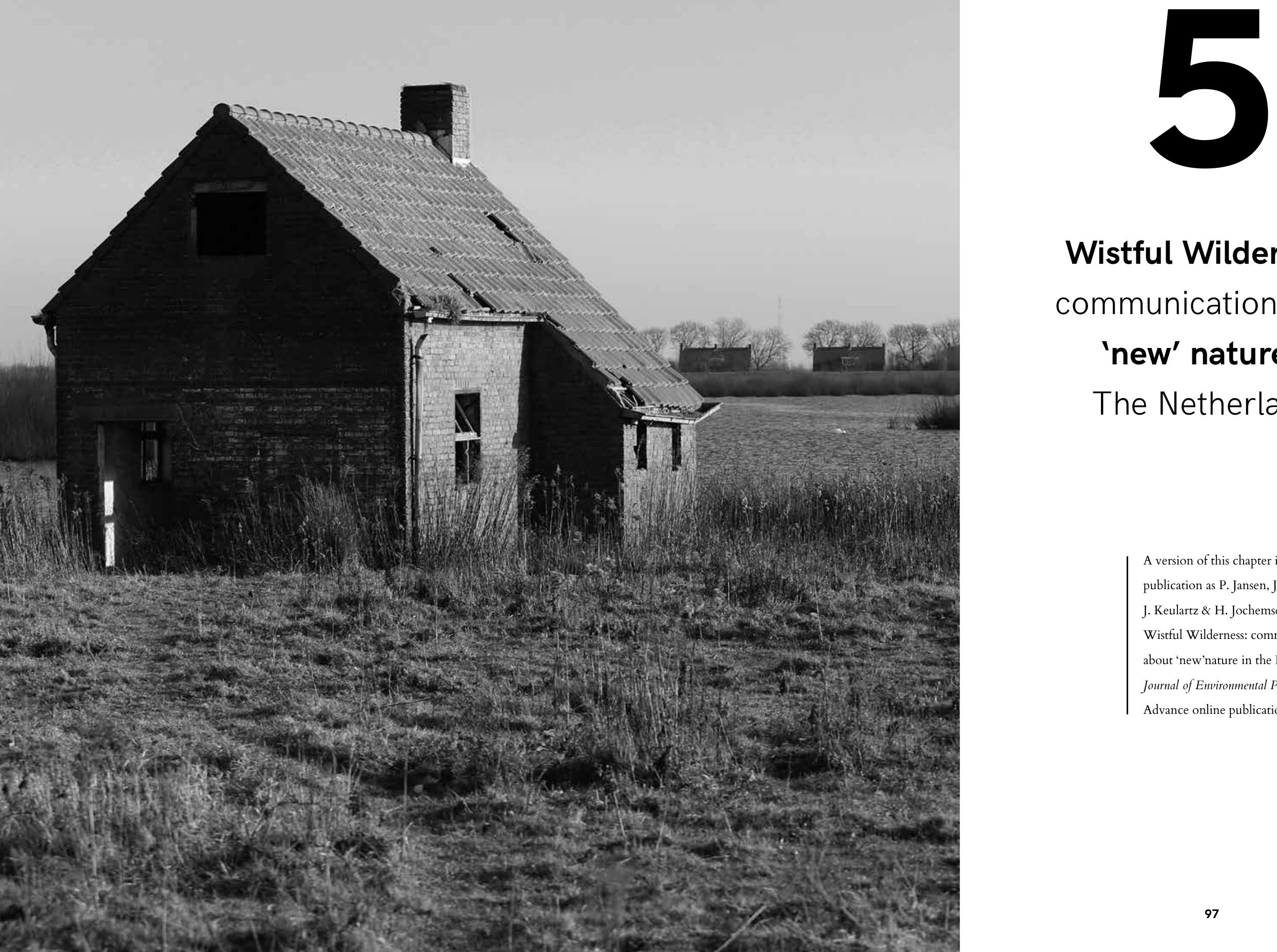

\section{Wistful Wilderness:}

communication about 'new' nature in The Netherlands

A version of this chapter is accepted for publication as P. Jansen, J. van der Stoep, J. Keulartz \& H. Jochemsen (2016). Wistful Wilderness: communication about 'new'nature in the Netherlands. Journal of Environmental Policy \& Planning. Advance online publication. 


\section{Wistful wilderness:} commmunication about 'new' nature in the Netherlands

ABSTRACT - Based on in-depth interviews, this chapter presents findings of a study centred on public communication regarding Tiengemeten, a Dutch island previously occupied by farmers. An answer is sought to the question of how visitors to Tiengemeten evaluate, according to their own experiences, the discourse of people involved in Tiengemeten from a policy and communication perspective. This study showed that visitors' experiences do not always match the emotions appealed to in public communication materials. It is also suggested that people involved from a policy and communication perspective should refrain from using 'heavily value-laden' phraseology. For reasons of trust, this chapter suggests aligning public communication with genuine experiences of visitors. This is also necessary for avoiding scepticism of visitors as policy makers and communication professionals run a risk that public communication regarding nature becomes counterproductive.

\section{INTRODUCTION}

In the Netherlands until the 1980s and 1990s, the nineteenth-century agrarian landscape was popular. However, the Dutch government concluded that Dutch

86 Cf. Deacon et al. (2010). In this chapter, we use purposive sampling, also known as typical-case sampling. This means that the main topic is leading by selecting the area of study. The goal of this type of sampling is not to maxi-

mize external validity by means of a random sampling, as in quantitative research, but to interpret a theme by utilizing a selected example. nature had limited ecological sustainability as a result of substantial habitat fragmentation. Hence, the Dutch nature policy plan of 1990 intended to compensate for the inadequate connectivity by creating a coherent network of nature reserves throughout the Netherlands. In this nature policy plan, Tiengemeten, the subject of thi chapter, was referred to as one of these (new) nature reserves which implied a transformation from agricultural land into a nature island (photos 1 and 2). ${ }^{8}$ 

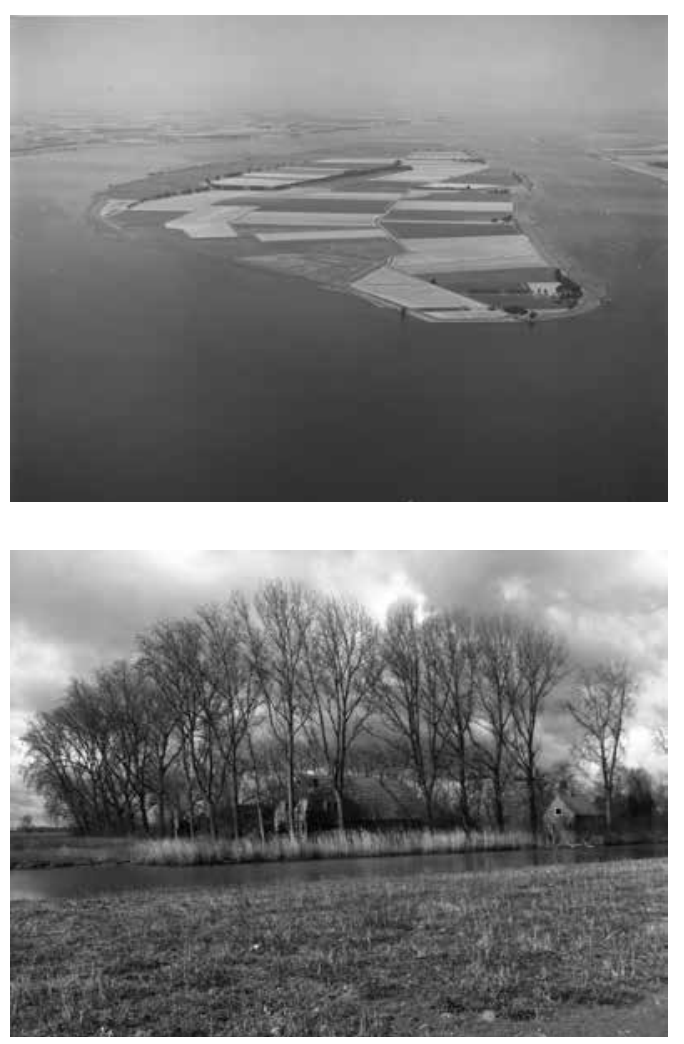

Photo 1. Aerial photograph:

the island before transformation.

(Source: https://beeldbank.rws.nl

Rijkswaterstaat / Joop van Houdt)

Photo 2. Wistful wilderness: the

nature takes over the farmhouse.

Tiengemeten is a small island in the south-western part of the Netherlands. It was formed as a consequence of the sedimentation of a sandbar in the Haringvliet between 1750 and 1804. The area was diked in 1750 and became the domain of farmers, fishermen, reed cutters, and hunters. After the Second World War, Rotterdam began its restoration and claimed Tiengemeten as a perfect destination for industrial expansion or a suitable location for a marina, an airport, or a sludge depot. In 1967, AMEV, an investment company, purchased the island as an investment project. Since then, other designations for Tiengemeten ranging from an airport to a waste depot battled for priority. However, in 1994, the Provincial Authority of South-Holland designated Tiengemeten as a nature development area with the intention of creating a nature island. After functioning as an agricultural area for a few hundred years, the island would be made a part of the network of existing and future nature reserves throughout the Netherlands. In 1997, one of the Dutch nature conservation organizations, Natuurmonumenten, became the owner of Tiengemeten. Following a comprehensive study and plan development that was completed in 2000, the transformation of Tiengemeten into a nature island began in September 2005. The dikes around Tiengemeten were destroyed in order to allow the sea complete freedom and allow nature to take its course. In 2006, the last of seven farmers left the island and the transformation was completed in 2007 (photos 3 and 4).
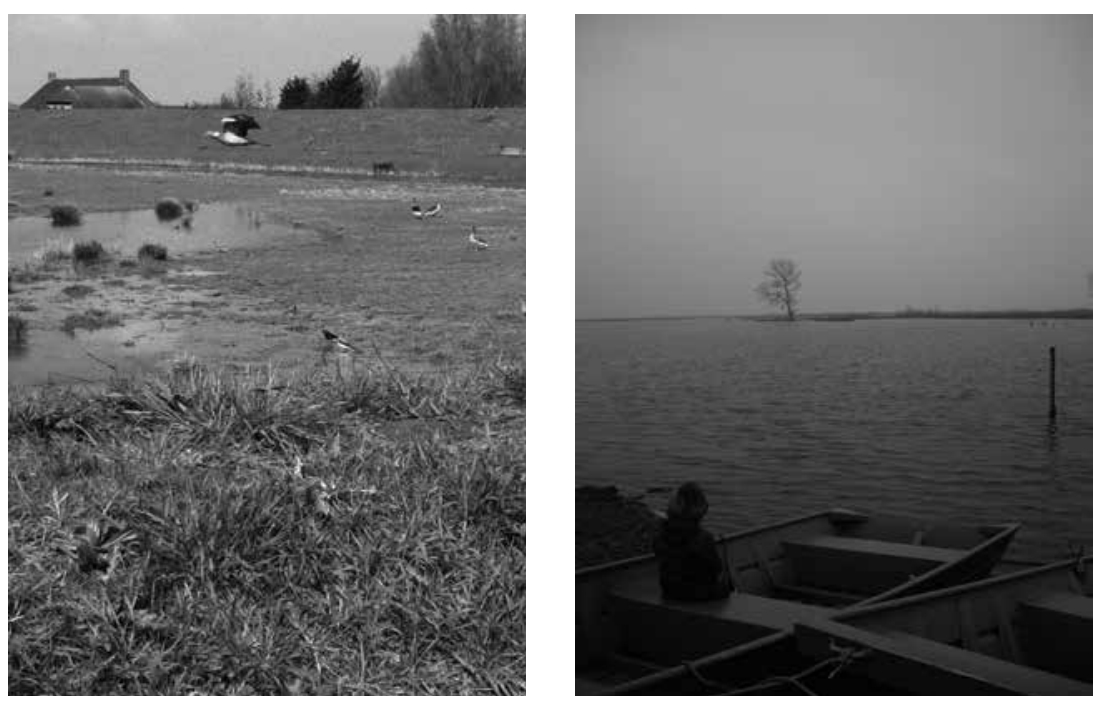

Photo 3. Wealth-part: an abundance of birds.

Photo 4. Wilderness-part: influence of the tides is dominant, place for rest and tranquility seekers.

When developing Tiengemeten, the planners decided to divide the island into three zones with various names. On the eastern side of the island there is a relatively small area called weemoed (wistfulness). Here, the starting point is the memory of the (cultural) landscape of the past. In the area designated as weelde (wealth), human influence is only minimally visible; it is an open marsh with an abundance of (water) plants and birds (e.g. Anas crecca, Tadorna, Recurvirostra avosetta, Numenius arquata, and different geese). The third and largest section of the island is referred to as wildernis (wilderness). In this area, the influence of the tides of the Haringvliet is dominant. This area is not being managed and is characterized by 'disorder, wild, exciting, expansive, special types of physical exertion' and 'especially for naturalists, rest and tranquility seekers and "nature wanderers" this type of nature is most meaningful' (Posthoorn, 2000:61). In order to further clarify the three distinct areas, they are characterized by sets of qualifying words as exhibited in Table 1. 
TABLE 1. Characterizing three parts Tiengemeten

\begin{tabular}{|l|l|l|l|}
\hline Part & Wistfulness & Wealth & Wilderness \\
\hline Description & Cultural landscape & $\begin{array}{l}\text { Semi-natural } \\
\text { landscape }\end{array}$ & Natural landscape \\
\hline Qualifying & Memory & Wealth & Tension \\
words & Security & Harmony & Fear \\
& Human & Beautiful & Authenticity \\
& Designed & Calm & Wild \\
& Readable & Understandable & Autonomous \\
& Stable & Tranquility & Disorder/Chaos \\
& Trust & Colorful & Dynamic \\
& Contrast & & Blurred boundaries \\
& Hospitable & & Inhospitable \\
\hline
\end{tabular}

Source: Posthoorn (2000), translation by authors.

This chapter intends to provide insight into the public communication regarding transformation projects such as Tiengemeten. ${ }^{87}$ Interestingly, Wolf (2012) contends that Tiengemeten is one of the few nature reserves where the term wilderness is explicitly expressed. It is obvious that, in public communication, Tiengemeten is considered as being representative of a 'new way of thinking' and that 'heavily value-laden language' is utilized. ${ }^{88}$ The following quote concisely illustrates this:

Tiengemeten: new nature, breaking ground like the water of its creeks. The symbol of new thinking, of trust in the shaping capacity of nature. Primeval nature, nature that seems to have been ever-present (quote in leaflet Natuurmonumen ten, s.a.).

Based on qualitative research, this chapter addresses the question to what extent the discourse of key actors corresponds to visitors' descriptions of their experiences of Tiengemeten. In order to answer this question, the discourse of people who were either involved from a policy perspective in the transformation of Tiengemeten or played an appropriate and influential role in the public communication, so-called key actors, must first be examined. Secondly, how visitors evaluate the discourse about Tiengemeten, in regard to their own experience of nature on the island, must be
87 In this chapter, we mean all types of communication dedicated to inform people about a certain nature development project by public communication, for example, leaflets, information booklets, billboards, etc. See Table 2 and the methods section of this chapter for further information.

88 We borrow the term 'heavily value-laden language' from Hobbs et al. (2014). investigated. The final section of this chapter will compare both and discuss the implications of the findings for (communication) policy and strategy.

\subsection{THEORETICAL FRAMEWORK}

Having briefly sketched the outline of the case, the position of the study in the broader field of nature policy and nature conservation will be described by reflecting on other studies in this field addressing actual debates about perceptions of naturalness and/or new ecosystem concepts.

\subsubsection{Nature development}

One of the key principles of the Dutch nature policy plan of 1990 is the concept of nature development (chapter 4). This refers to the creation of 'new' nature through human intervention or, as stated by Hajer (2003):

It has resulted in a slow-motion ballet méchanique (sic) of draglines and bulldozers, excavators and trucks. Under the direction of engineers and geomorphologists fertile topsoil is dug away to make way for wetlands or drifting sand, dikes are breached to create new lakes or 'living rivers' and at carefully chosen places dunes are opened up to allow the sea, the 'water wolf' that haunted the Dutch for centuries, back in (Hajer, 2003: 90, emphasis in the original)

The creation of 'wild' nature is a concept that Schouten (2002) considered to be novelty in Dutch history. Some authors refer to a radical shift in attitudes: one that respects 'exalted primeval nature' and criticizes the 'view of nature of the [Dutch] Golden Age (17th century) and that of modern engineers' (Zwart, 2002: 46). In the past, the idea of 'unspoiled wilderness' was recognized in existing nature reserves that were protected from human influence; however, the current desire for wilderness serves as justification for human interventions in the context of the creation of 'new' nature (Drenthen, 2003) of which Tiengemeten is a good illustration. In general, nature development facilitated a new way of thinking about nature as well as a change in the mentality regarding spatial planning and zoning in the Netherlands (chapter 4). The Netherlands is considered to be a man-made country, and the repurposing of land for nature development is consequently me with resistance. In March 1993, The New York Times published an article with the significant title 'Dutch Do the Unthinkable'. This article states: 
In a nation where historically nothing could stand in the way of creating more arable land, the Dutch turnabout strikes many experts as remarkable. The plan has wide support in the cities, but stirs concern across the countryside where farming families have worked the land for generations.

The concept of 'nature development' is generally associated with 'restoration ecology' (chapter 4). According to Jackson and Hobbs (2009), this type of ecology refers to 'ecological history as a means of identifying appropriate restoration targets - the state of the ecosystem before disruption - and assessing sources of damage' (567). ${ }^{89}$ However, it is contended that restoration to a historic standard is anachronistic: 'undisturbed landscapes are too remote in time to provide restoration targets, which may instead comprise cultural landscapes' and 'environmental and ecological changes are normal' $(567)^{90}$ or, as stated by Hobbs et al. (2010):

In the realm of park and wilderness stewardship, the natural world has been contrasted with the human-dominated world. In this sense, two related characteristics of naturalness are a lack of human effects on ecosystems and an absence of intentional human control of ecosystems. Interwoven with this has been the notion that natural ecosystems are stable, self-regulating, and in equilibrium. A fourth commonly perceived characteristic of naturalness has been a high degree of historical fidelity; natural ecosystems should appear and function much as they did in the past. This had led park and wilderness-area managers to use past conditions as benchmarks for the future (Hobbs et al., 2010: 485)

They conclude that naturalness has too many meanings which increasingly conflict with each other and 'fall short of capturing the expanding range of park values' (Hobbs et al., 2010: 485). Therefore, they prefer the term intervention 'to include any prescribed course of action that intentionally alters ecosystem trajectories, and to avoid the connotation of a return to past conditions' (484). Jackson and Hobbs (2009) note that 'restored ecosystems may have combinations of species that have never co-occurred' (568). They maintain that such ecosystems must be embraced as 'an unstated aim in restoration is to avoid creating bigger problems than we seek to solve' (568). We are now in an era of transformation, Holling (2001) argues, and
89 Hobbs et al. (2010) argue that: 'people have increasingly questioned the naturalness and feasibility of maintaining natura conditions in park and wilderness areas. [ ... ] With increasing recognition of the potential effects has come awareness that it may not even be desirable to maintain the historical conditions we often associate with naturalness' (483).

90 They note that historical studies remain valuable in determining ecosystems' structure and function before disruption. ecosystem management must build and maintain ecological resilience as well as possess the social flexibility required to cope, innovate, and adapt. Hobbs et al. (2009) conclude that many ecosystems are currently being rapidly transformed into what is referred to as 'new non-historical configurations' as a result of several local and global changes. Hence, 'novel ecosystems' are defined as ecosystems 'in which the species composition and/or function have been completely transformed from the historic system' (601). ${ }^{91}$ In another article, Hobbs et al. (2006) note that novel ecosystems can be considered as somewhere 'in the middle of the gradient between "natural": or "wild" ecosystems, on one hand, and intensively (agriculture) managed systems on the other hand' (2). In their vision, there is a need to move away from what they term 'the one-dimensional dichotomy between natural and human dominated' (5) ecosystems 'towards a more effective depiction of how humans interact with nature' (5). This challenges policy makers on how to develop management schemes that simultaneously maximize beneficial changes and reduce the less beneficial aspects (Hobbs et al., 2006), viz. 'a combination of traditional and emerging frameworks' (Hobbs et al., 2014: 557). According to Hobbs et al. (2014), novel ecosystems provide opportunities 'to connect with nature for a wide cross-section of society' (561); therefore, it is time to stop thinking in terms of 'second-class nature' in regard to these types of ecosystems.

91 According to them, novel ecosystems are closely related to

hybrid ecosystems, but 'the hybrid hybrid ecosystems, but the hybrid ered the state in which most of the measurable traits of the ecosystem (i.e. nutrient load, hydrology, species diversity, etc.) are the same but most of the species have changes. The novel state would then be defined as when measurable traits are altered from historical ranges' (Hobbs et al., 2009: 601).

$92 \mathrm{He}$ elucidates this as follows: 'the frame, a robust 30 -foot-wide picture frame made of scaffolding poles and painted beams, stood at the edge of the fields framing the view of passers-by. Thanks to the astute sense for symbolic politics on the part of the farmers the point

was hard to miss: many people in the local community actually felt attached to the landscape as it was' (Hajer, 2003: 91).

\section{.2.2 Classic-modernist practic}

It is interesting that Hobbs et al. (2014) reason that novelty itself demands broad public dialogue' and 'careful discussion is required on appropriate goals for such systems' (561). At the same time, they also note that describing novel systems with 'heavily value-laden language' will 'increasingly marginalize conservation and restoration in the public realm' (562). In this context, it is striking that Hajer (2003) discusses that the issue of nature development in the Netherlands illustrates 'the limited effectiveness of classical-modernist political practices' (89). Based on the case of Gaasterland where, in the early 1990s, farmers erected metal frame in the field as a protest, he concludes: ${ }^{92}$

The policy conflict [ ... ] was not merely based on different notions of beauty of the Dutch rural landscape. In actual fact it brought out into the open the 
limits of the classic-modernist practices of politics and policymaking, more in particular the institutionalised format in which social interests get represented [ ... ] The frame in the fields first of all confronted the government with the limited effectiveness of its traditional strategies of 'conceive-decide-implement' in a network society. [ ... ] The protests were not anticipated in the predominant neo-corporatist practices of consultation at the national level [ ... ] It did not merely concern the content of policymaking but the practices of policymaking as well (Hajer, 2003: 91, 92+95).

This is in accordance with authors such as Swart et al. (2001) and Keulartz (2009). They argue that the Netherlands continues to struggle with the implementation of 'new' nature because citizens do not determine the direction of nature policy but, rather, this is delegated to researchers and professionals. There is a discrepancy between the professional-based (ecological) knowledge upon which nature policy has heavily relied and local, experience-based knowledge, that is, local perceptions and opinions. Otherwise stated, the resistance against the repurposing of land for nature development is first concerned with the practice of policy-making, or more precisely, the difficult implementation, that is, realization of a national ecological network throughout the Netherlands foremost appears to be a communication issue.

\subsection{METHODOLOGICAL APPROACH}

As mentioned in the introduction, central to this chapter is the question to what extent the discourse of key actors corresponds to visitors' descriptions of their experiences of Tiengemeten. However, the term discourse is used 'in a range of meanings' (Hajer \& Versteeg, 2005). Hence, it is necessary to clarify what is understood by 'discourse'.

\subsubsection{Defining discourse}

As Tannen et al. (2015) note, because of its disciplinary diversity, 'it is no surprise that the terms "discourse" and "discourse analysis" have different meanings to scholars in different fields' (1). This chapter has adopted Hajer's (1995) description of discourse in terms of Keil and Debanné (2005) 'a more or less non-controversial definition' (258). Hajer (1995) defines discourse as an ensemble of ideas and concepts that are 'produced, reproduced and transformed in a particular set of practices' (44). Interestingly, Hajer and Versteeg (2005) conclude that, in everyday life, the terms discourse and discussion are often interchangeably

used. However, they argue that both should be analytically distinguished, 'the "discussion" $[\ldots]$ is the object of analysis; discourse analysis sets out to trace a particular linguistic regularity that can be found in discussions or debates' (175). According to them, discourse analysis 'illuminates a particular discursive structure that might not be immediately obvious

93 According to them, there are two primary pproaches of discourses. 'The main cleavage runs,' according to Feindt and Oels (2005), 'between Foucaultian and non-Foucaultian concepts of discourse' (163). And 'most non-Foucaultians ly to symbolic interactionism' which means that it 'focusses on the linguistic and pragmatic produc tion of meaning' (163). In contrast, the Foucaultan perspective on discourse 'is more interested in knowledge than in language' (164). It is more about power construction because 'language is not a neutral messenger of given interests and preferences, but it influences their very formation' (166). Cooren (2014) also distinguishes two types of discourses: with an uppercase ' $D$ ' (see FootNote 95) and with a lower case ' $d$ '. The latter regards 'what people are up to when they communicate which each other (what they do and how they do what they do) as well as how the conversation itself functions and organized' (7).

94 Feindt and Oels (2005) note that the concep

of environment relates humans and society 'to the "natural" systems with which they interact and to the "natural" sources they use', including 'nature protection' (164).

95 This is what Cooren (2014) identifies as the Discourse with an uppercase ' $\mathrm{D}$ ', 'It means that you are able to recognize its typical form or content, as well as its typical context of production' (5). According to him, it is also noteworthy that 'people who are reproducing specific Discourses can literally be seen as their carries, which means that we could almost say that not only are these persons expressing themselves when they are talking, but also the (typical) Discourses they represent'(5).

96 Cf. Dingler (2005) contends that it is impossible to make a statement about nature 'without a reference to the discourse in which the statement is made' (214). Interestingly, Cronon (1995) notes that this does not mean that nature is unreal or ' mere figment of our imaginations - far from it',

however, the manner in which we describe and understand nature 'is so entangled with our own values and assumptions that the two can never fully separated' (25). to the people that contribute the debate' (176). They consider discourse analysis as 'the study of language-in-use' (Hajer \& Versteeg, 2005: 176). Or in terms of Feindt and Oels (2005):

Discourse analysis problematizes what conventional policy analysts take for granted: the linguistic, identity and knowledge base of policy making. This includes a special awareness of the processes by and through which policy roblems and even policy arenas are constructed. A discourse analysis of policy making shows how environmental problems and a related set of subjects and objects are discursively produced and rendered governable (Feindt and Oels, 2005:163).95 Interestingly, Feindt and Oels (2005) argue that, in the environmental policy area - and in our view there is no reason why this should not apply similarly to the field of nature policy ${ }^{94}$ - problems are typically not defined in common sense language, but in expert terms of reference' (162). ${ }^{95}$ It is also argued that the articulation of a problem 'shapes if and how the problem is dealt with (162). This accords with Svarstad et al. (2008) who contend that statements, for example, about nature, should be considered as 'articulations and reproductions 
of discursive orders' (119). ${ }^{95} \mathrm{He}$ also argues that communication regarding these issues does not emerge from scratch or by a single communication actor. 'Actors involved in a discourse participate - in varying degrees - in its production, reproduction and transformation through written or oral statements' (119). More precisely, according to Feindt and Oels (2005), concepts that are part of a discourse are intertwined with or are a part of practices, institutional capacities, and technologies. As they note:

They have material and institutional bias. They have also a history. They are rooted in specific cultural and political formation. They enable people to see and articulate certain features of the world but not others. They help to legitimate certain practices but not others. They are an element of power formations (Feindt and Oels, 2005: 163).

To conclude, inspired by Hajer and Versteeg (2005) and applied to this topic, investigating particular discourses could be advantageous for tracing how a concept such as nature development (i.e. the wilderness ideal) first emerged as a key principle of the Dutch nature policy and how its meaning subsequently evolved as it was molded by institutional settings and through the application on particular cases.

TABLE 2. Public communication

\begin{tabular}{|c|c|c|}
\hline Type & Quantity & Source \\
\hline Leaflet & 9 & Natuurmonumenten \\
\hline Member Magazine & 1 & Natuurmonumenten \\
\hline Billboard Visitor Centre & 1 & Natuurmonumenten \\
\hline Booklet & 1 & Ministerie van LNV \\
\hline Booklet & 3 & VVV Zuid Holland Zuid \\
\hline
\end{tabular}

\subsubsection{Data collection and analysis}

This chapter does not intend to search for a particular linguistic regularity or discursive structure. Consequently, in the narrowest sense of the word, no discourse analysis is performed. Instead, a specific situation is examined in regard to how ideas and concepts are employed and, hence, the term discourse is justifiable in this context. Semi-structured, in-depth interviews are chosen as the primary instrument of data collection for reasons of triangulation (cf. Du Plooy, 2001) supplemented by quotes from public communication (see Table $2, n=15$ ). The selection of respondents $(n=25)$ resulted from 'criterion-based sampling', that is, the selection of respondents was based on predetermined 'criteria': (i) visitor to the island, whether or not regular and (ii) people involved from a policy perspective in the transformation of Tiengemeten into a nature island or an appropriate and influential party in the communication, so-called key actors. In this study, visitors were personally recruited on the island. During the recruitment process, discussion occurred with the potential respondents regarding the topic of the focus of the research. This afforded the opportunity for each respondent to think more deeply about the topic and accurately recall memories. Within 2-4 weeks after consenting to an interview, an appointment of approximately one hour was made to be held at the visitor's home or at a pre-selected location (for instance a restaurant or local bar). In total, 15 visitors were interviewed.

\section{TEXTBOX 1: Visitors of Tiengemeten}

Since the quality of nature and the quality of enjoying nature are foremost, Tiengemeten has become an island that attracts a wide range of people. The planning process took into account three types of visitors: curious people (nieuwsgierigen), nature lovers (natuurliefhebbers), and naturalists (natuurvorsers). The curious people arrive on the island, have something to eat, walk to the Vliedberg, take in the area, and return home. They spend no more than two hours on the island. The second category, nature lovers, mostly travel east on rambling trails. The third category, naturalists, is relatively small. These are people who actually go into the wilderness area primarily to experience nature. Research by de Boer and de Vries (2009) indicates the numbers of visitors range from 30,000 to 40,000 per year. According to them, on average, visitors to the island are 52 years old and are mainly from a radius of $30 \mathrm{~km}$ around the area. The average group size is five individuals, although half of the visitors travel in pairs. The majority visit to either relax or walk. Since the realization of a so called 'nature playground', many children are also currently visiting the island. Natuurmonumenten also mentioned on their website that there has been an increase of visitors between 10 and 29 years old. The island has increasingly become a destination for a family to have a day out. General speaking,

Tiengemeten, focuses on two (main) target groups: active seniors and families with young children (between 4-12 years of age).

Key actors were recruited by so called 'snowball sampling' which offers a method for identifying and contacting hidden research subjects: one subject provides the 
researcher with the name of another subject who subsequently provides the name of a third, and so on (Vogt, 1999). In total, 10 key actors were interviewed. A whole overview of the discourse was pursued, hence, the composition of the group key actors is multifarious. ${ }^{97}$ Most of them are employees of Natuurmonumenten - a Dutch NGO for nature conservation and owner of the island Tiengemeten that is responsible for most of the public communication.

Contrary to quantitative research, the goal of qualitative research is not to maximize external validity, and it does not provide insight into the relative number, that is, percentage of people in the group that represent a certain conception. ${ }^{98}$ However, with qualitative research, the sample size is a point of attention and, according to Mason (2010), the guiding principle by interviewing is data saturation. Although the concept of data saturation is not new and is generally accepted, Mason (2010) mentions a continuing debate while Fusch and Ness (2015) argue that 'the field of data saturation is a neglected one' (1408). The reason is that this concept is diffcult to define. They note that what one individual considers as data saturation is not nearly enough for another. For that reason, they contend that 'it may be best to think of data in terms of rich and thick rather than the size of the sample' (1409). As they explain:

Thick data is a lot of data; rich data is many-layered, intricate, detailed, nuanced, and more. One can have a lot of thick data that is not rich; conversely, one can have rich data but not a lot of it. The trick, if you will, is

to have both (Fusch and Ness, 2015: 1409).

Therefore, Fusch and Ness (2015) come to the conclusion that data saturation is not foremost about numbers but about the depth of the data. Interest-
97 The key actors include the developer of the Tiengemeten $v$ sion (respondent 16), the project leader of the transition (responden 17), a film and documentary maker who filmed the transition of the island for over thirteen years (respondent 18), a general communications employee for Tiengemeten (respondent 19), the Tiengemeten visitor manager (respondent 20) the employee who is ultimate $r$ sponsible for the communication about Tiengemeten (responden 21), the mayor of Korendijk (the municipality in which Tiengemeten is located, respondent 22), the designer and developer of the 'nature playground' on Tiengemeten (respondent 23), the Tiengemeten nature conservation officer (respondent 24), and the last farmer to leave the island (respondent 25). The fi farmer (respondent 25) have been excluded from our analysis as the are not directly associated with Tiengemeten from a policy or communication perspective. They were only a relevant party to speak with for obtaining a complete picture of the discourse. Nevertheless, the tithe of this chapter is derived from a documentary made by the film maker in 2010.

98 With qualitative research, is difficult to ensure that the sam results can be replicated. Baarda al. (2009) argue that it is problematic to repeat qualitative researc under the same conditions because the many factors that play a role on location cannot be controlled. The argue that 'validity and reliability of data and decisions based upon these aspects are typical quality criteria for quantitative research (7). They indicate that qualitative research is more about terms such as credibility, transparency, an adequacy. ingly, Mason (2010) argues that there is no logical (or theory driven) reason why samples ending in any one integer would be any more prevalent than any other. He notes that, if saturation is the guiding principle of qualitative studies, it is likely to be achieved at any point, and most authors agree that saturation could be achieved at a comparatively low level. ${ }^{99}$ In our case, saturation was achieved with approximately $12-15$ (visitors) and 8-10 (key actors), that is, 20-25 interviewees.

TABLE 3. Core labels and key categories.

\begin{tabular}{|c|l|l|}
\hline \# & Core labels & Key Categories \\
\hline I & Project & $\begin{array}{l}\text { Project, Policy-making, Transition, Impact of decision, } \\
\text { Activities and Facilities. }\end{array}$ \\
\hline II & Experiences & $\begin{array}{l}\text { Appealing aspects of Tiengemeten, Effect of island on } \\
\text { people, Recreative content of island. }\end{array}$ \\
\hline III & $\begin{array}{l}\text { Perceptions } \\
\text { of nature }\end{array}$ & $\begin{array}{l}\text { Nature development in the Netherlands, Views about Ag- } \\
\text { riculture, Views about Nature, Experiences with nature, } \\
\text { Transcendent aspects, Immanent aspects. }\end{array}$ \\
\hline IV & $\begin{array}{l}\text { Communi- } \\
\text { cation }\end{array}$ & $\begin{array}{l}\text { Communication strategy, Parties involved in communi- } \\
\text { cation, Process of communication, Promotion of island, } \\
\text { Communication materials, Words and Images, Evocative } \\
\text { aspects. }\end{array}$ \\
\hline V & $\begin{array}{l}\text { Notions of } \\
\text { meaning }\end{array}$ & \begin{tabular}{l} 
Sense-making, Spirituality, Religious elements, 'Zeitgeist'. \\
\hline
\end{tabular} \\
\hline
\end{tabular}

The interviews were recorded in order to obtain a detailed analysis of the text. Interviews were analysed according to standard text analysis. Prior to examination of the interviews, an analysis level needed to be selected, therefore, a combination of sentences and fragments were chosen (Baarda et al., 2009). Consequently, the stripped or summarized texts were divided into small fragments expressing a similar theme or content and labelled with a code, that is, keyword (open coding, 259 pcs). In the next phase, the coded or labelled sections were compared for similarities and differences and grouped around key categories or sublabels $(25$ pcs). In the synthesis and abstracting (axial coding) 99 He concludes that the most phase, overarching core labels (5 pcs) were defined; common sample sizes in research see Table 3 - for reasons of intersubjectivity and peer papers were 20 and 30 (followed consultation the entire process of coding is discussed ture reviews, Mason (2010) derives with other scholars in several (international) meetthe following numbers: ethnogra- ings. Finally the results were analysed and linked to phy 30-50 interviews, grounded the research questions (selective coding); this chapter views, and phenomenology 5-25. presents the results of this phase. 
Since our primary focus was on public communication, extensive discussion on the core label 'experiences' were not addressed in this paper. The input with respect to this core label is partially utilized in $₫ 5.5 .3$; most of the other input was used for another chapter (chapter 6).

\subsection{RESULTS DISCOURSE OF KEY ACTORS}

\subsubsection{Perceptions of natur}

In the beginning of the transformation process, respondents 16 and 17 indicated that communication was generally simplistic: 'ask farmers to leave the island, demolish all houses, put a hole in the dyke, and nature will be there soon'. As illustrated by the quotation below:

Tiengemeten is an island in the Haringvliet, where agriculture have to make room for nature [ ... ]. The island Tiengemeten will change significantly: from a polder (a piece of low-lying land reclaimed from the sea and protected by dikes, $\mathrm{PJ}$ ) with agriculture into a nature area with swamp and forest (quote in the booklet Ministerie van LNV, 1994).

Respondent 24 noted that, in his opinion, a number of errors were made at the beginning. The concept of 'allowing water on the island' in the public communication was interpreted as 'submerge the island'. He feels that this instance of poor communication, to this day, determines the image of the place. This makes the question regarding the perception of nature of the key actors crucial. According to respondent 22:

In the beginning the values of nature dominated [communication materials] [... ] the nature paradise on earth. 'Come and see this' but not really because it's a gem we have to protect.

When asked about their personal perception of nature, most of the key actors associated nature with something that involves a minimal amount of human interference. According to respondent 23:

I'm the kind of guy who likes to look for that one tiny blade of grass in between the tiles of the sidewalk. That's nature. I mean, that's also the essence of nature: the fact that it occurs where you'd think it would be impossible. It's the enigmatic dynamic of life, of always growing and being there no matter the odds $[\ldots .$.$] . To me that's nature: it always brings some-$ thing new. It's that endless and inexhaustible cycle.

At the same time, all key actors realized that much of nature in the Netherlands has been influenced by humans or consciously conserved or developed. Consequently, respondent 16 regards Tiengemeten as a 'significant cultivation act', as landscape that was designed and made by people rather than by 'original' nature, and a place where the influences of mankind can still be detected but where nature has gradually begun to take over. The best thing, according to respondent 16 , is when people no longer have the idea that their surroundings are contrived or overly humanly designed.

\subsubsection{Communication}

As mentioned in the methods section of this chapter, when developing Tiengemeten, the planners decided to divide the island into three zones and give the various areas some type of iconic title, that is, the triple-W concept. According to respondents 16,17 , and 24 , this was a conscious decision as it sounds much better than technical jargon such as 'rainwater-driven area', 'cultural-historical area', or 'tidal area', respondent 17 said. While respondent 16 enjoys the alliteration, Key Actor 22 would not mind if the three Ws were discarded:

just think: well, wilderness is clear-cut. But what exactly is wistfulness and wealth? So, yeah, as concepts they don't do very much for me [ ... ] It's terminology you also see at the visitor centre, but it doesn't really work. It doesn't match the experience.

Most of the key actors argue that words such as wealth, wistfulness, and wilderness will, by definition, evoke a certain sentiment; the concepts appeal to certain emotions and, according to respondent 16 , consider them as powerful labels that have had more effect than originally intended.

Several key actors note that having a meaningful or appealing topic, such as 'new nature', that is, Tiengemeten, is crucial in public communication. Speaking in regard to a core message in public communication, several key actors discuss the soul of Tiengemeten - 'a little rough and raw' as well as 'clouds, wind, and water' being an element. Respondent 21 believes that 'rough and raw' encompasses 'the exciting aspects' such as the ferry, the ruins, and what happens on the island. Public communication should foreshadow how visitors will feel once they are there. 
I mean, you want something spectacular. So that's what you look for. A white-tailed eagle, size of an aircraft, two-metre wingspan - it's big. [ ... ] So this attracts you, like, on a different level. [ ... ] It's something that transcends, that's bigger. [ ... ] In the communication already they suggest how you might feel without ever even having been there. So reading the texts, about a 'majestic eagle', it almost transports you to that world and you think 'wow, so that's what I'd feel!' (respondent 21).

The majority of key actors feel that the promotional material should generally, first and foremost, evoke the feeling of 'that's where I need to be; that's what I want to experience'. They believe that, in terms of respondent 21, public communication should affect people and 'move them' and 'generate buzz'. The language should not be too superficial or polished but should offer some type of 'seductive' prospect - an 'interesting' proposition, stated respondent 21. Respondent 19 also stated:

think we're all convinced that they (terms such ecological infrastructure, connection zones, etc., PJ) don't resonate with Joe Six Pack. So we have to use other words, other concepts. But what interests me is that often these terms are very existential. 'I roam around nature [ ... ] the term 'primordial' already says a lot, I think.

[ ... ]

For example, there's that huge roll-up in the visitor centre, with 'longhaired beasts' and the 'savage' creatures pictured on it - I mean, 'savage', they're very friendly and that is subsequently what you try and explain: they're very sweet animals, they [only] look savage.

Respondent 16 does not believe that 'cold' information exists, stressing that the request for information is always emotionally driven. However, he notes that there is continuous tension to ensure that things do not become 'too kitsch'.

When asked about current public communication materials on Tiengemeten, a number of key actors indicate that they find it fairly down-to-earth when compared to other public communication materials about nature that are issued by the same association. Respondent 16 noted that there is a significant amount of public communication and material on what he refers to as 'the outside', that which we are physically able to see, that is, the various plants and animals, etc. However, for instance, respondents 19 and 21 stated that, in recent years, the public communication material regarding Natuurmonumenten has become increasingly focused on experience and attracting visitors. In the past, Natuurmonumenten was quite factual and cerebral; however, according to the respondents, in recent years, there has been a move towards experience. According to respondent 19, communication concerning Tiengemeten is also more about 'come and see' or 'experience the island'; there is a primary focus on the fun aspects of rambling and enjoying oneself on Tiengemeten as illustrated by the quotation below:

Human-sized brush and grassland, dissected by a dozen streams. Highland cattle with their legs in the water and a gap in the dike. Thousands of birds of all kinds and sizes. Chaos and silence. This is the kind of wilderness we have missed. This is Tiengemeten the primeval delta now! [ ... ] It's quiet in the Wilderness. Quiet and yet bustling with life [ ... ] The hill is the best vantage point to catch a panoramic view of the Wilderness, the Haringvliet with its locks and fixed embankment on either side. A safe place 'separated from the world' (quote in leaflet Natuurmonumenten, s.a.).

However, the quote above also emphasizes another aspect, specifically, that Tiengemeten represents 'the wilderness we have missed' and that the island is 'separated' from daily life. In this respect, respondent 16 's comment is intriguing:

A lot of people don't come to Tiengemeten to think about things, but rather to let go of their thoughts.

According to respondent 17 , this is reflected in (public) communication:

What one sees in the means of communication is the contrast between the fast-track life of the Randstad and the tremendous bustle of Pernis and the Port and the vast tranquil open spaces not even twenty kilometres away.

This brings us to the use of notions of meaning in the discourse about Tiengemeten.

\subsubsection{Notions of meaning}

In this chapter, 'notions of meaning' are utilized as an overarching term for aspects of sense-making as well as spiritual elements in general - in literature the term 'religious subtexts' is also used in this context (chapter 4). The quotes below demonstrate that there is a strong appeal to 'an escape from daily life' in the current public communication about Tiengemeten where it is referred to as being 'the better world' with silence presented as an instrument for spiritual experience. ${ }^{100}$ 
Everyone needs to get away from the grind of everyday life, don't they? So why not take a trip and discover an island of tranquillity, space, and distant horizons (quote in leaflet Natuurmonumenten, 2012).

Looking to enjoy the tranquillity of the starry night sky? Then the island of Tiengemeten is the perfect place for you. Nature has been allowed to run its free course over the last few years. Tranquillity and open spaces characterize the island, leaving the bustle of the Randstad far away. Tiengemeten is a popular location for day trippers looking for a relaxing day out. But you can only really unwind once on a lengthier stay [ ... ] Leave behind the fasttrack life of the city and come to rest. Spend the night on an uninhabited island, watch the sun rise over the Haringvliet, take a twilight walk while the birds float down to search for a nesting place. Take in the night sky as you've seldom seen it (quote in booklet VVV Zuid Holland Zuid, 2012).

Where in today's world is there a place where the horizon stretches fur ther than the eye, where there are no footpaths, and visitors can ponder amidst the birds? Where silence is audible, open space is tangible and you can come to rest? Where the waters are wild and untamed [ ... ] feeding and drowning? [ ... ] The world is miles away, worries melt and a smile breaks through (quote on billboard at Tiengemeten visitor centre, Natuurmonumenten).

Often, some type of promise is made: recharge, find yourself, purify, a new world, etc. One of the key actors discussed nature as a place from which to return reborn.

The nature island in the Haringvliet is the place to recharge. [ ... ] The ferry voyage already gives you a sense of shedding the pressure of everyday life. And once you disembark on Tiengemeten, you're setting foot on a new world (quote in Member Magazine, Natuurmonumenten, 2011: 20).

In this case, it is interesting that, in these types of projects, respondent 17 suggested that the decision-makers often prefer to use someone who, although not specially trained in communication and who perhaps may be awkward or less refined than an educated communication professional, does have some affinity with nature. In this context, the following quotation is intriguing:
100 In 2009, the government asked for a vacancy to be filled with a communication professional who is able to bring the experience of silence to the attention of the public - one of the ideas is to promote Tiengemeten as an icon of silence.
If you ask them (people working at Natuurmonumenten, PJ) about their earliest experience of memory related to nature from their childhood, they often come out with very existential and emotional stuff (respondent 16). ${ }^{10}$

With that in mind, it is unsurprising that a number of key actors note that their individual experiences with nature define the way in which they communicate about nature. 'I really do believe in what I write every time,' respondent 21 said. 'They're all little bits of myself.'

\subsection{RESULTS OF VISITORS' OPINIONS}

\subsubsection{Perceptions of nature}

When asked about their perception of nature, several visitors felt that nature is typified by self-construction and freedom. Some visitors cite the importance of creating conditions for natural processes to thrive and expand. In this context, some visitors argue for an absence of or minimum human interference. Other visitors feel untouched nature or wilderness are synonymous with 'true' nature; 'true' nature actually refers to 'pure' with the meaning of 'spontaneous' and 'autonomous' rather than 'original'. The pureness for some visitors, in regard to projects such as Tiengemeten, is due to the fact that, although the site is the result of 'landscaping' work, it is subsequently left to develop naturally. In this case, certain visitors also mentioned the dynamics of the tides at Tiengemeten.

\subsubsection{Communication}

It is striking that certain visitors (for example, respondents $2,4,5,6,7$, and 11) did not do much research on Tiengemeten prior to their visit. Some came for the nature experience and were not initially really interested in communication. However, when delving deeper, the public communication about Tiengemeten, for most of them, unconsciously stimulated them to visit the island. When asked about the striking aspects, the visitors mentioned the experiential aspect of the public communication. Interestingly, respondent 8 noted that this aspect is closely associated with the current zeitgeist (spirit of the age).

01 This is in accordance with Taylor's (2001) observation which We live in the fast lane, everything needs to be a is that most members of the Amer- quick, all-inclusive experience. So you need to have have had some kind of mystical multiple stimuli.

experience in nature' (179). 
Some visitors, for instance respondents 11 and 14, emphasized wilderness, especially at the beginning of the transformation process, and the relinquishing of the island to nature. However, it is notable that, in order to promote Tiengemeten and show that there are still pieces of 'primeval' nature in the Netherlands, respondent 8 feels that it must be advertised much like Disneyland is advertised. Stated differently, according to some visitors, the communication is sometimes a bit 'over the top'. Some respondents $(4,8$, and 15$)$ feel that current communication about nature is a little too jovial considering that nature itself is not 'polished and nice' but also involves threat and struggle. For instance, respondents 8 and 15 question whether people are employing terms that are inappropriate. The addition of 'true' to 'nature' in public communication is perceived by some visitors, for instance respondents 5 and 15 , as being pointless and misleading and as being related to the level of aspiration: people wish it were so (respondent 15).

In contrast to the key actors, visitors are more critical about the triple-W concept. Some visitors are wonderstruck (respondent 8 ) or argue that the words do not resonate appropriately (respondents 2 and 5 ). At the same time, other visitors note that they consider the three Ws to be a positive choice as 'they stick with you' (respondent 10). Respondent 11 feels that such labels incite individuals' curiosity. One visitor (respondent 8) felt they acted as a filter for the general public about what to expect, feeling it was an attempt to associate the desires of the average tourist to an emotional experience.

From this perspective, it is interesting that respondent 3 believes it is important to have communication that matched visitors' actual experiences on the island. Whether communication materials appeal to something specific for the visitors depends on the visitors' actual experiences which must correspond to the emotions that are evoked. However, for instance, respondents 1, 3, 5, and 8 note that the words and images used in public communication do not always resonate: there is a tension between the words used and the actual experiences on Tiengemeten. In other words, emotions that may be evoked or appeal do not always correspond to actual experiences on the island.

\subsubsection{Notions of meaning}

With regards to the attractive characteristics of Tiengemeten, the island's spacious character - the empty and expansive countryside that is enhanced through seeing clouds and feeling the wind in combination with a sense of freedom - is mentioned by most of the visitors. Interestingly, for respondent 1 , the old landscape of
Tiengemeten appears to be crucial to his experience; he perceives the Tiengemeten of the past reflected in the Tiengemeten of now. Respondent 1 as well as respondent 15 yearned for the previous rural life of Tiengemeten:

Tiengemeten was the ultimate polder feeling. I love the polder whether it's winter or summer or autumn; this polder is always beautiful (respondent 1).

Traditional countryside, agrarian countryside, in the end is simplicity [ ... ] Exceptionally beautiful farmland (respondent 15).

Several visitors (respondents 8,11 , and 15) cited that the experience of the place was determined by Tiengemeten being an island; the ferry trip was regarded as a unique experience as it fostered feelings of being 'on your way to another place'.

When asked about their personal experiences with nature, a number of visitors referred to nature as helping them to obtain a balance in life; nature seems to be a place to mentally recuperate (respondents 3 and 11). Nature is considered as an ideal setting to vent frustration and to help an individual achieve inner peace (respondents 2 and 4 ) or, according to respondents 4, 10, and 15, nature is 'the other side of the coin':

For people nature is the other side of the coin. The opposite of the urban jungle, of busy, stress, work, misery, mortgage, the whole shebang [ ... ] nature means tranquillity (respondent 4 ).

Respondent 4 cites the ruggedness of nature and its changes as the source of the insight that things are relative, transient, and never inert. Some visitors also believe that nature has the power to take you back to your roots; it is a source of inspiration and can touch your very core (respondent 4). It is interesting that most of the visitors are cautious about discussing these types of experiences with regard to Tiengemeten. However, respondent 11 talks about a ritualistic dimension, that is, ritualistic in the context that it is a regularly recurring action to break free from the burdens of everyday life.

For a moment you're taken out of that little world. You get on the boat and travel towards the next step. [ ... ] Then you're aware that 'on this island it's all about nature - that's priority number one'.[ ... ] It becomes a kind of sacred space. Nature becomes something holy and clearly demarcated and protected. That space, that is. There's not very much to say: the island is just there. 


\subsection{DISCUSSION}

In regard to perceptions of nature, our study indicates that there are no significant substantial differences between the visitors and the key actors; the majority of both groups refer to nature as something that develops autonomously and preferably without (much) human interference. Stated differently, according to our respondents, 'real' nature is 'untouched nature', that is, wilderness. Our findings are in accordance with other empirical studies. For instance, Buijs et al. (2009) note that Dutch people, in general, are strong supporters of wilderness nature. A study conducted by Van den Born (2008) ascertained that, where Dutch individuals' opinions on (real) nature are concerned: 'people are very clear about this: the extent to which nature is cultivated' (92). According to her research, people are a part of nature but have their specific place and responsibilities within it. 'It is a paradox, but not an opposition. [ ... ] responsibility means to give space to flourish and respect nature's autonomy' (Van den Born, 2008: 103+104). De Groot and van den Born (2003) also note that most respondents in the Netherlands take a non-anthropocentric attitude to nature; they conclude that responsibility for nature is clearly part of the current Dutch mainstream culture. Despite the consensus among respondents that wilderness is the most 'real' form of nature, it appeared that the majority of the visitors were cautious about exploiting the terms 'true' or 'real' nature in relationship to Tiengemeten. The outcome of the transformation process of Tiengemeten is primarily considered as 'a cultivation act', and it is difficult to determine a clear distinction: 'if you use the phrase true nature, then that implies there's also such a thing as fake nature. And I don't feel that that exists' (respondent 5). If it is possible to refer to something as 'real' nature, then the only the area of the island that can be labelled as 'wilderness' is where the tides freely affect the island because nature has begun 'to take over' in that location. Drenthen (2003), however, notes that, when we ourselves create 'wildernesses', 'wildness' can no longer be considered as a standard of the 'realness' of nature. Our study demonstrates that 'realness' refers to 'spontaneous' and 'autonomous' rather than 'true' or 'original'. This finding has implications for (communication) policy and strategy because the use of the term 'real' nature to mean 'true' or 'original' dominates the discourse in the Netherlands. Our study shows that the wilderness ideal is prominent in the discourse of key actors in regard to Tiengemeten and concepts of meaning are significantly evident in the booklets, leaflets, etc. However, it appears that current public communication about nature intends to overly promote 'wild' and 'untouched nature' as 'true' or 'original'. This study suggests that it is better to avoid such words with a 'heavily value-laden language' as these do not necessarily match the experiences of visitors.
A number of key figures argue that public communication should suggest in advance how visitors will feel once they are on Tiengemeten; public communication should evoke the feeling of 'that's where I need to be; that's what I want to experience'. However, this study indicates that experiences on the island do not always correspond with the emotions evoked and appealed to in communication materials, that is, words and images that are used in the public communication about the island. More precisely, our study shows a discrepancy between the discourse of the key actors' and visitors' descriptions of their experiences of Tiengemeten. Instead of wandering through the wilderness with a sea eagle circling overhead, they experience a savanna-like landscape with excessive wind and children playing around them. The question then arises as to whether the key actors in their discourse have taken into account the experiences of visitors and/or the actual situation of the island which is a (nature) development project subject to continuous change. As a result, the preferences of visitors are also constantly changing. Our study shows the urgency for (communication) policy and strategy to consistently align their public communication on the actual state of the development of the nature projects. This is important for reasons of trust which is, in our opinion, a mixture between truth (is what I say correct) and sincerity (are my intentions right, am I honest). However, it is also necessary for avoiding scepticism of the visitors as you may run to risk that public communication becomes counterproductive.

Hence, in general, it can be concluded that visitors evaluate the discourse of key actors as not being adequate. This results in the following key insights, in this case, implications of our findings for (nature) policy and (communication) strategy:

- Be careful using words such as 'wild' and 'untouched nature' in the sense of 'true' or 'original'. Avoid 'heavily value-laden language' in public communication about nature.

- Evoked emotions in public communication do not automatically correspond with the actual situation and experiences of visitors. For that reason, avoid pious words and false promises; however, tell visitors what the nature reserve actually brings.

- Change is inherent to projects of nature development and hence all public communication is tentative. Each stage requires various words, images, etc. This leads to the recommendation that visitors' actual experiences of the nature reserve should be incorporated more into the communication about 'new' nature projects. As a result, public communication would be perceived 
by visitors as less 'pompous', and the discourse of the key actors would then be more in accordance with the descriptions used by visitors when expressing their experiences of 'new' nature.

- Despite the points above, words and images in the discourse of the key actors could function as a framework for visitors' experiences; these words and images could be used by visitors to express their experiences as one of the findings was that visitors struggle with the articulation of their experiences.

Addressing the points above is an attempt to make a contribution to the research of public communication regarding nature development projects. This chapter hopefully elucidates the understanding of visitors' communication needs in relationship to their individual experiences. This chapter is a first attempt, hence the character of this study is explorative; further in-depth and additional quantitative research is required.

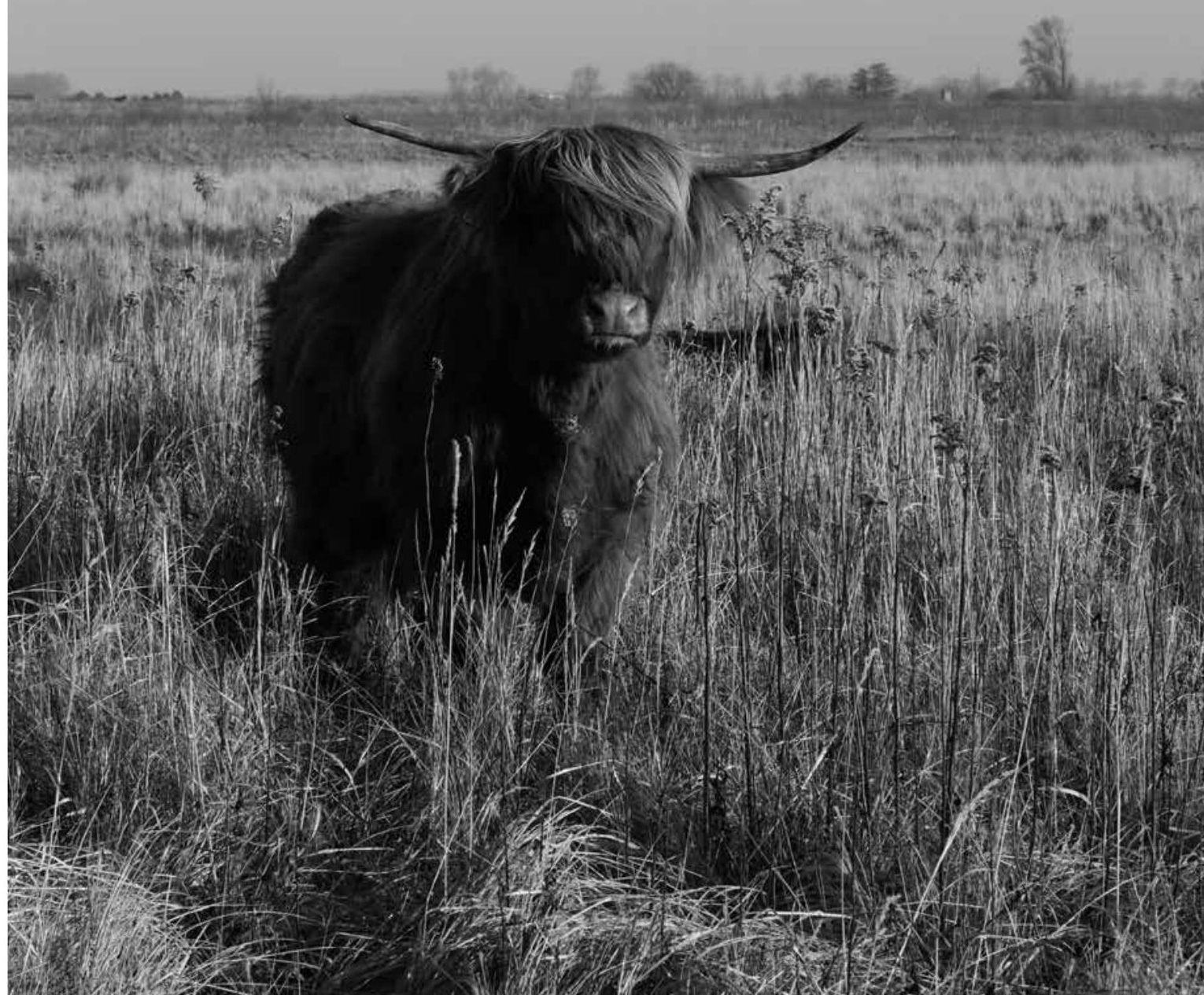


Notions of the Sublime:

A Case Study into Layers of Depth in

Experiences of Nature

ABSTRACT - A re-experiencing of the wilderness ideal is taking place in the Netherlands. This is expressed in the attention that is being devoted to projects in which agricultural land is transformed into nature. One of these projects is central to this chapter: the island Tiengemeten. We aim to study the experiences of visitors to this project in order to gain insight into the extent to which there are layers of depth visible in these experiences. For a lot of respondents going into in nature in general and making a visit to an island such as Tiengemeten in particular are ways of escaping from their day-to-day existence. It is noticeable that the words and images that the visitors use to describe their experiences fit in mainly with the wilderness view of nature. The wilderness view of nature is connected with experiences of the sublime; so in this chapter we also ask the question as to what the shift to the wilderness view of nature means for the religious landscape of the Netherlands.

\section{INTRODUCTION}

For many years nature organisations in the Netherlands maintained an Arcadian view of nature (Van der Windt et al., 2007). This view of nature focuses on rural nature, on nature that is characterized by a man-made landscape where patterns from the past are observed (Keulartz, 2000; Van Koppen, 2002). However since the eighties, the wilderness view of nature has become popular in the Netherlands (Buijs, 2009). According to this view, nature as such has an untamed and pristine character and is considered to be valuable because of being something primordial. Wilderness refers to uninhabited and uncultivated spaces, to an 'environmental system where natural processes occur with little or no significant influence by human beings' (Taylor, 2012:293). 
Ferguson \& Tamburello (2015) argue that what they called natural amenities is 'a resource that meets the spiritual needs of human' (2). ${ }^{102}$ According to them the natural environment is 'a resource that could be used for facilitating spiritual 'feelings, acts, and experiences' (3). ${ }^{103}$ Champ (2009) also concludes that nature is a source of 'deeply meaningful experiences one might call 'religious' or 'spiritual' (226). ${ }^{104}$

Nature has the ability to be imbued with spiritual power and significance. Forests, lakes, and mountains often invoke a feeling of the divine or inspire a sense of awe. They are a resource that people may use to connect to the sacred and to generate spiritual feelings

(Ferguson \& Tamburello, 2015: 3-4). ${ }^{105}$

The aim of this chapter is to use a case study to gain greater insight into the significance of nature experiences and views of nature in the life of nature conservancy area visitors. Hence the central question of this explorative chapter is: what layers of depth can be identified in the description of the experiences of visitors to Tiengemeten?
102 They follow Sewell (1992) in his description that 'objects, animate or inanimate, naturally occurring or manufactured, (...) can be used to enhance or maintain power' (9). This power is according to them 'spiritual power and energy' (3).

103 They follow James ([1901] 1999) in his definition of religion and argue that James' definition reflects the current popular understanding of spirituality. James ([1901] 1999) defines religion as 'the feelings, acts, and experiences of individual men in their solitude, so far as they apprehend themselves to stand in relation to whatever they may consider the divine' (36).

104 Ferguson \& Tamburello (2015) note that the terms 'religion' and 'spirituality' are not mutually exclusive. However they argue that 'religion' 'may be thought of as institutional, mediated, and communal' and 'spirituality' 'points to the personal, subjective, non-institutionalized, and unmediated experience with the sacred' (3).

105 Droogers (2010) defines the sacred as anything related to a separate, exceptional, different, direc tive reality that is experienced as above and beyond people.

\section{TEXTBOX 1: Scope of Study and Methods}

Tiengemeten is an island in the south-west of the Netherlands that was recently transformed from an agricultural island into a nature island. The island is divided into three spheres or zones. On the eastern side of the island there is a relatively small area called 'wistfulness' (Weemoed). Here, the starting point is the memory of the (cultural) landscape of the past. In the area called 'wealth' (Weelde) human influence should be barely visible. This part of the island is characterized by a limited control of natural processes and the main aim is to maintain the different types of nature. The last and largest part of the island is referred to as 'wilderness' (Wildernis). This area is not managed at all and the influence of the tides is dominant.
This chapter presents data from a qualitative case study conducted in 2012 In total 25 people were interviewed. For the selection of respondents we used 'criterion-based sampling', i.e. the selection of respondents was based on predetermined 'criteria': (i) visitor to the island, whether or not regular and/or (ii) people involved from a policy perspective in the transformation of Tiengemeten into a nature island and/or an appropriate and influential party in the communication, so-called key actors. In this chapter we focus primarily on visitors. Interviews were analysed according to standard text analysis. Consequently coded or labelled sections (259 pcs) were compared for similarities and differences and grouped around key categories, in this case sublabels (25 pcs). At the end we defined overarching core labels (5 pcs). In this chapter we focus on only one of the categories, i.e. 'experiences'.

In order to answer the central research question we look, in the first part ( $\$ 6.3)$ of this chapter, at how visitors experience Tiengemeten. This part has a descriptive character. In the second part $(\$ 6.4)$ we look at what types of experiences can be identified in this description. This part has a categorising/analytical character. Finally in the third part ( $\$ 6.5$ ) of this chapter we look to which extent layers of depth can be distinguished in the experiences described. This part has an interpretative character.

FIGURE 1

Three-part structure of this chapter

\begin{tabular}{c} 
I \\
\hline DESCRIBE
\end{tabular}$\rightarrow$ CATEGORIZE $\rightarrow$ III

At the end of this chapter $(\$ 6.6)$ the various findings are linked and are discussed in light of the central question. This leads us to briefly deal with the question what the results of our study imply for the religious landscape of the Netherlands. However, we will start off with a short theoretical sketch $(\$ 6.2)$ regarding views of nature and the layers of depth to be distinguished within it.

\subsection{THEORETICAL FRAMEWORK}

The Dutch discourse on nature is a mixture of romantic understandings of Arcadian nature and technical understandings of wilderness nature. The Arcadian view 
of nature is based on weak anthropocentric values, i.e. the stewardship worldview, as opposed to the wilderness view of nature, which is based on strong biocentric values, i.e. an ecocentric worldview. Based on Keulartz et al. (2004) and Buijs (2009), we define the following characteristics for both views of nature (see table 1). We will use this table in the last part in this chapter in the interpretation of the empirical data.

\section{TABLE 1 Comparison of views of nature}

\begin{tabular}{|l|l|}
\hline Arcadian nature & Wilderness nature \\
\hline - Man in harmony with nature & - Nature in contrast with man \\
$\quad$ (weak-anthropocentric) & (eco-/biocentric) \\
- Experiencing the beauty of nature & - Experiencing unpredictable nature \\
- Sympathy for historical elements & - Sympathy for sublime elements \\
- Nature is fragile & - Nature is an ecosystem \\
(community ecology) & (system ecology) \\
- Emphasising conservation & - Emphasising nature's own course \\
- Modelled upon stereotypes of na- & - Modelled upon stereotypes of na- \\
ture, i.e. picturesque, Romantic, & ture, i.e. primeval, spontaneous/ \\
pastoral, rural idyll, etc. & autonomous, true nature, etc. \\
\hline
\end{tabular}

Buijs et al. (2009) refer to views of nature as 'cognitive reflections of prior experiences with and discourses about nature' (114). Drenthen (2002) too makes a link between views of nature and experiences. He states that various interpretations are preceded by 'the appealing experience of nature' (78) and that our various interpretations are an answer to this appealing experience. In other words, experiences gained in nature influence a person's view of nature, which implies that views of nature can be identified in what people say about nature. But the opposite also applies: a person's views of nature influence their experience(s) of nature. Figure 2 shows this diagrammatically.

\section{FIGURE 2}

Relationship between experiences and views of nature

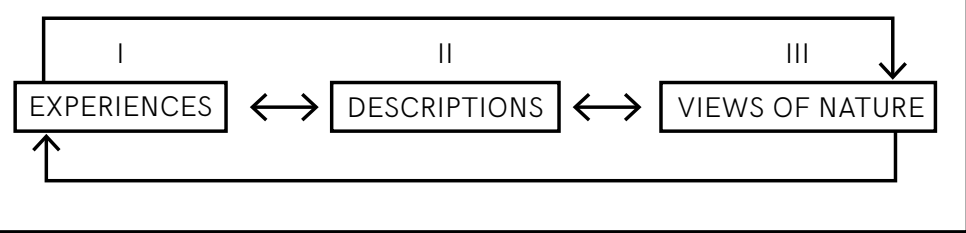

By describing our experiences we become aware of these experiences : when the experience is expressed in language, a reflection is implicitly carried out.
Describing experiences creates a certain distance with regard to the concrete experience (Roothaan, 2005). This is to say that articulating experiences is a form of objectification. Or, to put it another way, language has an objectivising effect with regard to our experiences. But expressing experiences in language makes it possible to share them with other people. By making our experiences shareable we attach personal, subjective meaning and sense to them. Meanings occur not in a person alone but between people (Linell, 1998). Talking about experiences and/or sharing images defines and deepens experiences. Other people can also recognise themselves in these shared experience(s). Which means that communication about nature can also evoke experiences.

Expressing experiences in words means that what has been experienced can (in a distilled form) be included in future experiences, by other people, in other places. In this way personal experiences can be picked up by other people whom I do not know, for reasons that I do not know or share (Roothaan, 2005: 54-55).

In short, by communicating about our experiences of nature things are not only described and imagined (articulated); they are also defined and given meaning (categorized and interpreted).

With regard to disclosing meaning, 'language constructs' such as frames play a role. Frames navigate our thoughts, they structure our process of perceiving, i.e. our experiences. To understand how frames are related to depth dimensions in communication the distinction between surface frames and deep frames is helpful (Lakoff, 2006). Surface frames function at the level of our daily language. They make clear what the communication is about and provide us with a point of view. However, surface frames appeal to underlying values and convictions that can be communicated in deep frames. Deep frames connect our daily language with our normative convictions regarding the world and our lives. In the context of this study we understand the Arcadian view of nature and the Wilderness view of nature as deep frames. 
FIGURE 3

Relationship between experiences and views of nature

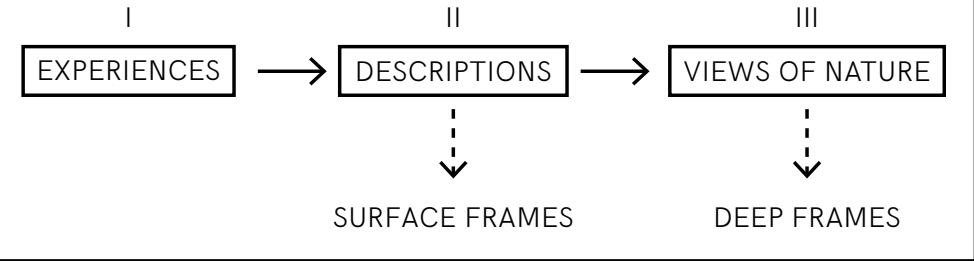

Figure 3 summarizes the theoretical framework in diagrammatic form and this figure serves as a 'backbone' for this chapter.

\subsection{EXPERIENCES}

In this first part we look at how visitors experience Tiengemeten. In other words, why do people go to Tiengemeten, what do they actually experience and gain from it? Several visitors primarily expect people with a love of nature and the countryside to visit Tiengemeten. Nevertheless several respondents assert that a varied audience, from all over the Netherlands, visit Tiengemeten, with a number of respondents referring mainly to 'curious people' as visiting the island. ${ }^{106}$

Regarding the key attractive characteristics of Tiengemeten, the island's spacious character - the empty and expansive countryside enhanced through seeing clouds and feeling the wind in combination with a sense of freedom - and relative tranquillity are cited frequently by a lot of the respondents (photos 1 and 2). Mass tourism, overscheduling of activities and 'overly well-kept' sites tended to diminish the experience for some visitors in general and the experience of nature specifically. For some visitors a distinct recognition of the old landscape of Tiengemeten seems crucial to their experience. They see the 'Tiengemeten of yore' reflected in the Tiengemeten of now; the former expression crops up regularly during the interviews. It refers primarily to the more or less isolated character of the island, described by some as a 'free state' and as a place where the environment of modern life is far less present than in the rest of the Netherlands. One or two individuals found the Tiengemeten of yore to be more beautiful than that of the present. 'Tiengemeten
106 The planning process took into account three types of visitors: curious people (nieuwsgierigen) nature lovers (natuurliefhebbers) and naturalists (natuurvorsers). The curious people arrive on the island, eat something, walk to the Vliedberg, take in the area and go home. They spend no more than two hours on the island. The second category, nature lovers, mostly go east on rambling trails. The third category, naturalists, is relatively small. These are people who actually go into the wilderness area and primarily come to experience nature.
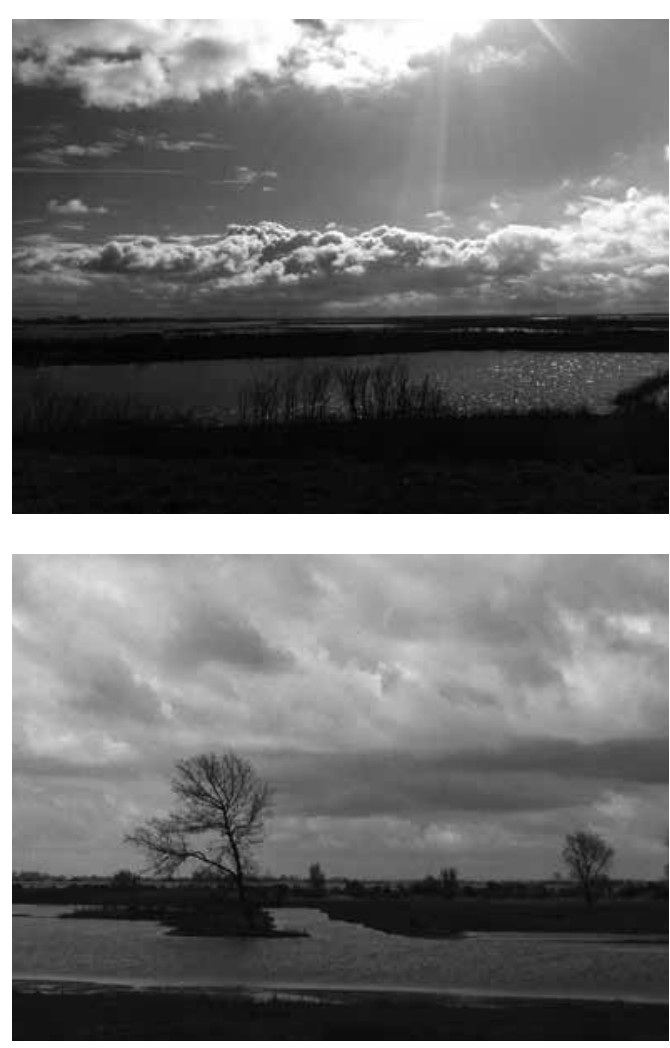

Key attractive characteristics: seeing clouds (photo 1) and dynamics of the tide (photo 2).

was the ultimate experience of the polders' (respondent 1). The old Tiengemeten exuded the nostalgia of rural life. As respondent 15 puts in:

Traditional countryside, agrarian countryside, in the end is simplicity. Narrow roads just wide enough to fit a tractor. No highways, no road demarcations, no street names. I enjoyed that. There was something about it. And the cars that were around, well, they were ancient. I liked that.

Exceptionally beautiful farmland (respondent 15)

Several visitors cited the aspect of 'being on an island' as a particularly attractive factor. For a large number of respondents the experience of the place was determined by Tiengemeten being an island. Such experiences, however, are nothing new. Even in the past when Tiengemeten was just an island used for farming, the ferry trip itself was regarded by several key actors as a unique experience. However, some key actors feel that the ferry is now used more strategically, as a means to give a visit to Tiengemeten a specific flavour. 
It is also striking that various respondents mention the dynamics of the tide; but ruggedness and unspoilt nature are also mentioned in relation to Tiengemeten. Most visitors associate nature with 'letting it happen' and 'letting things take their course', such as 'nature takes its course, that's what I call wilderness' (respondent 7). The fact that it was people who started the process, as far as Tiengemeten is concerned, did not meet with many objections. ${ }^{107}$ It was created but for respondent 10 there is nothing wrong with that as there is now 'movement'. There were conditions set out so that the 'natural process is given space' (respondent 4) and nature can 'establish itself' (respondent 12). According to respondent 13 you now get an idea of 'how it once was'. For several visitors this was the reason for visiting Tiengemeten, as here nature had been returned to 'how nature could be' (respondent 7). This also applied to respondents 8 and 11, who wanted to visit Tiengemeten as here 'nature had been returned to true nature' (respondent 8 ) and 'true wild nature with a direct influence exerted by river and sea' (respondent 11). Respondent 13 commented that 'wildness [was] as expected'. One respondent was sceptical: 'they would like it to be like that', 'there are too many traces of human intervention' and he therefore finds the term 'true nature' 'misleading' (respondent 15). For him nature is only true nature if it has always been nature. 'If cultural landscape becomes nature, it remains cultural landscape. It is just a different type of cultural landscape' (respondent 15).

\subsection{SURFACE FRAMES}

The experiences that can be identified in the visitors' descriptions of Tiengemeten can be classified into four types of experiences: (1) experiences of loss, (2) island feel, (3) experiences of escape and (4) experiences of grandeur. We will discuss each of the four categories below using the associated surface frames. We want to stress that this categorisation was done on the basis of the analysis of the texts of the interviews before we had a relationship in mind relating to a view of nature.

\subsubsection{Experiences of Loss}

Research shows that residents and tourists find the new landscape that was created by nature development attractive but at the same time feel less attached to it (Buijs et al., 2009). If a place changes, the attachment decreases, especially if the landscape is to be used differently: it is suitable for other activities and experiences than previously and logically it attracts different people (Van den Born \& de Groot,
107 Several respondents refer to 'park management' (respondent 8 or 'a bit of a fun park': 'I felt it was a bit of a fun park. Natuurmonument en often has really beautiful parks, very natural, but they are also a bit polished. Such a building is always natural anyway in which everythin is on display (...) but you also get al these Scottish Highlanders (....), it is simply a bit like a business card for Natuurmonumenten (respondent 11).
2011). This is in keeping with a comment by one of our respondents. One of the key actors noted namely that he expected increasing numbers of visitors from the whole of the Netherlands to come to Tiengemeten and that they would not have a lot of affinity (any more) with the past - 'they will see Tiengemeten as it is now and compare it with other areas of Natuurmonumenten' (respondent 19).
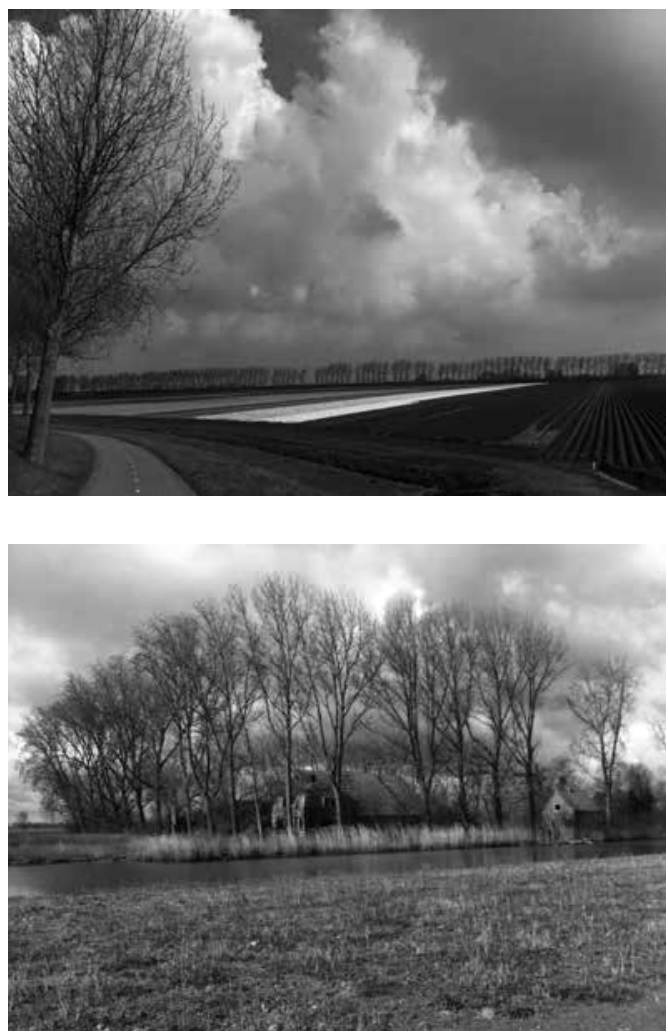

Before (photo 3) and after transformation (photo 4): loss of visibility historical origin?

Opposition to the development of nature arises above all from the loss of identity of the area and from a loss of the visibility of the history of the (agricultural) origins (Keulartz, 2011; photos 3 and 4). In the case of several respondents memories were reinforced at the sight of several former farms that remained. Some visitors still knew the names and functions of the various farms. The identity or individuality of the area along with the interweaving with personal life histories evoke feelings of familiarity with the landscape (Van den Born \& de Groot, 2011). At the same time dehistoricising the landscape results in a loss of identity (Keulartz, 2011). ${ }^{108}$ This also emerged in our study: visitors who 'have an affinity' with rural life or the island from the past were particularly sensitive to this topic. For some visitors it was important for the experience that the design of the old 
landscape could still be seen. 'You lose the design a bit of what it used to be,' said respondent 1 . And respondent 8 commented: 'there are elements that are a part of our history, there's no way of escaping that'. So some respondents see the old Tiengemeten in the new Tiengemeten. Although this is often to do with the relatively unique character of the island in the past - described by some as a 'free state' and as a place where there were fewer regulations than in the rest of the Netherlands. Follow-up questions showed that one person found the old Tiengemeten of the past more beautiful; see quote below by respondent 1 . The old Tiengemeten radiated the nostalgic rural life. In other words, for some respondents the past plays an important role in their experience of today's Tiengemeten.

Tiengemeten was the ultimate polder feeling. I love the polder; whether it's winter or summer or autumn, this polder is always beautiful. In winter it is neatly ploughed, or in autumn, and it is covered with snow, and in spring the new greenery sprouts and in summer there are the cornfields and the potatoes, a polder is always beautiful (respondent 1).

Attachment to a place is not just a result of one's own experience but also a result of stories heard or read (Thayer, 2003). We also found this in our study. Some respondents, who did not themselves have a direct historical link with the island, have trouble, for instance, with the farms being left to fall into disrepair. Not just from the point of view of 'the capital destruction' but also because of history, particularly because many generations had been born there and had grown up there.

You're born there and you want to live there for the rest of your life. Then because of something like this you have to move away (respondent 2).

They're now renovating a building. (...). The kids' drawings were still hanging in the attic. (respondent 10).

Although the focus of this chapter is on the visitors, it is interesting in this respect to see that the key actors (see Textbox 1) are properly aware of these experiences. Respondent 16 , for example, refers to a great sacrifice that had to be made. ${ }^{109}$ Respondent
108 Lemaire (2011) refers to inspired places if places are given a special meaning because we get attached to them. These places become a part of us over time. If they change, something in us is affected. 109 According to ser o several key actors, when the island was trans formed, buildings were left stand ing as a reminder of the fact that the transformation did not just happen by itself. According to several key actors, they really looked for a landscape that retained the story of people. 'In particular tha ruin of the big farm, where the las farmer lived. I thought something along the lines of 'everybody who visits it will feel a little uncomfortable. It has become a sort of mys terious, special place on that island (respondent 16).
19 comments that the emotions concerning the transformation are still felt: 'what happened still sticks to Tiengemeten' (respondent 19). ${ }^{110}$ According to respondent 21 the pain of the past - what we have called the experience of loss as a general term - is 'the island's soul' (respondent 21). ${ }^{111}$ In the classification of the island and official communications this can also be seen in the word 'wistful' (weemoed). ${ }^{112}$

\subsubsection{Island Feel}

As mentioned in part 1 of this chapter, the experiences of Tiengemeten are also influenced by the fact that it is an island. As a result of this some visitors experience Tiengemeten as a completely different world or as a completely different dimension of existence. The sight of it and the contrast with 'normal life' on the mainland reinforces this experience.

Life carries on there and I'm here and here life stands still for a while (respondent 8).

110 Respondent 21 comments that he is tired of being confronted with the 'farmer story' every time, 'it would be better to get rid of that now, it's over'. At the same time he recognises that this story exists and he struggles with how to ensure that it does not become too kitschy.

111 Respondent 22 comments that there are two sides to the is- but if you go by boat then it's suddenly very different land. 'On the one hand I see the beauty, on the other hand I see the pain there still is in the area. When you realise that a transformation process was initiated by the government that was not really supported by a lot of the population, you become aware that a lot of the people are still suffering when it comes to Tiengemeten. (...) I can't look at Tiengemeten in an unbiased way because I'm aware of the pain still felt by many people'.

112 In this sense, the title of an award winning documentary (Sinke, 2010) about Tiengemeten is well chosen: wistful wilderness. Seeing this documentary was one of the reasons to conduct this study.
Several visitors refer to a travel experience, which is reinforced by the crossing by ferry. Experiences of Tiengemeten are strongly influenced anyway by the necessary) crossing by ferry, as illustrated by the both quotations below.

What is normal [daily life, PJ] is on the mainland (...) It lets you escape from the hectic of life for a moment. (...) It's more like real life (respondent 15).

One of the visitors refers, in this context, to Tiengemeten as a 'sort of sacred space'.

ou step out of your own world for a moment, you get on a boat and then you move on to the next step. (...) You know that on this island it's all about nature, nature is the highest priority. (...) It is a sort of sa red space. Nature is there, it's sacred and it is very clearly embraced: this space, that's what it is. There in't that much to show: this island is just there (respondent 11). 
This quotation brings us by our third type of experiences.

\subsubsection{Experiences of Escape}

Whereas the first two categories were linked closely to the place, to the island of Tiengemeten itself, the next two categories of experiences about which the respondents talk are experiences that have to do with what nature does to the respondents. The aspect of escape/release is something we heard regularly. ${ }^{113}$ According to some visitors nature helps us to obtain balance in life.

I work in a knowledge-intensive organisation. And this is simply a completely different dimension; this helps me to achieve balance in my life, and that's what I use it for (respondent 3).

Nature is regarded as an ideal outlet that ensures that you can unwind.

For people nature is the other side of the urban environment, of bustle stress, work, misery, mortgage, the whole shebang. (...) nature means tranquillity (respondent 4).

Several respondents mention the ruggedness of nature and the changes to nature as the reason for the notion that things are relative. The emptiness and vastness of Tiengemeten have a positive effect.

You don't get that in many places in the Netherlands. There are places [on Tiengemeten, PJ] where, if you choose carefully, you see nothing at all of the real world. I really like that (respondent 5). ${ }^{114}$

Tiengemeten 'lets you escape from the hectic of life for a moment' and according to respondent 15 is therefore 'more like real life',

\subsubsection{Experiences of Grandeur}

Empirical research shows that most of the respondents (51\%) prefer nature where you can experience 'the greatness and forces of nature' (De Groot \& Van den Born, 2003: 134). In some experiences of nature the experience of one's own smallness compared with the grandeur of nature was regularly
113 The comment by one of the key actors is intriguing in this respect. 'A lot of people come to Tiengemeten not to think abou something but precisely in order to let go of their thoughts' (respondent 16).

114 The respondent refers with 'nothing' to an unobstructed and endless view. mentioned. One visitor refers to feelings of humility; one of the other respondents concisely describes similar feelings as follows:

I love it when we're sometimes reined in by a huge storm or a big thunderstorm. So that we realise that we're not as powerful as we think (respondent 23).

According to some visitors nature can take people back to their roots.

Nature is something primal, you go back to your roots in nature and then you relax, that's a great feeling (respondent 4).

According to several visitors you can get close to yourself in nature - you can lose yourself in nature and feel at one with nature. According to some visitors nature can stir you deeply and be a source of inspiration.

Whether you're religious or not, [in nature, PJ] there is always something that inspires you (respondent 4).

Some respondents state in general that people need spirituality and they find this in nature. ${ }^{115}$ One visitor sees 'God at work' on Tiengemeten.

I believe in God. And if you look at all that nature, we have nothing to do with it, but you see that Somebody was at work. And you also see, if you want to believe that, that a piece of nature is a bit of a 'paradise on earth' (respondent 10)

115 One of the respondents In this second part of the chapter we look at what kind comments that nature can be an of experiences can be identified in the experiences important (new) source of spiritual- described by the respondents. To summarize, there is a dichotomy, see figure 4. 


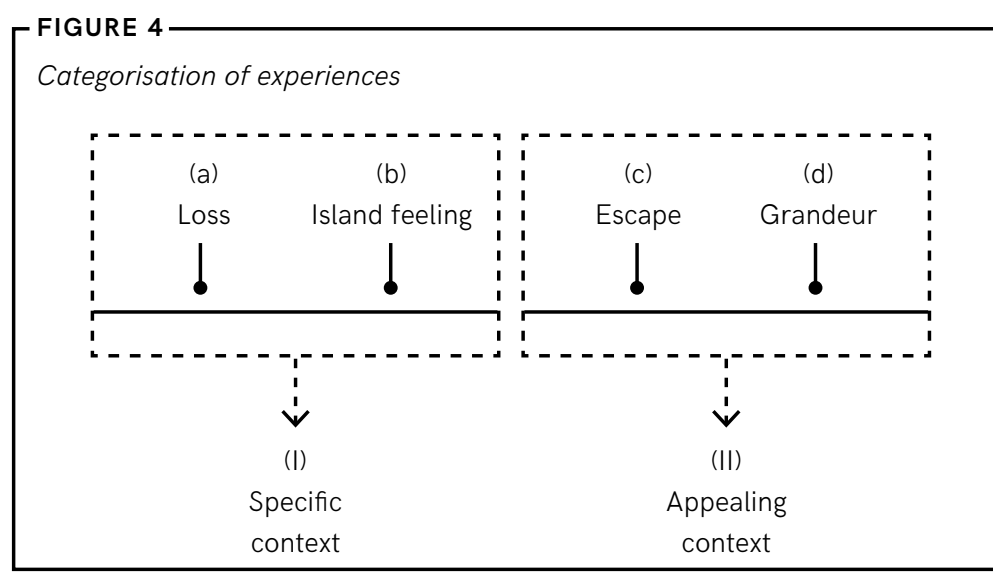

First of all (I) there are experiences that are strongly connected with the specific context. With regard to Tiengemeten these are (a) feelings of loss as well as (b) experiences to do with the fact that Tiengemeten is an island. Secondly (II) there are experiences that express the appealing effect of nature. These are experiences that have to do with (c) feelings of release and (d) experiences to do with grandeur. Interviews have shown that respondents who stressed the experience of loss were in general less positive with regard to wilderness. And respondents who made positive comments about wilderness had less trouble with the old farms on Tiengemeten falling into a state of disrepair. Using table 1 from the theoretical framework we want to look in the next section at what kind of layers of depth can be identified in the types of experiences referred to. In other words, in the third and last part of this chapter we make the transition from surface frames to deep frames and how these are connected to the two views of nature referred to above.

\subsection{DEEP FRAMES}

As mentioned in the theoretical part of this chapter, we understand the Arcadian view of nature and the Wilderness view of nature as deep frames. In the case of 'experiences of loss' experiences are described that fit with the Arcadian view of nature. Some respondents mention the loss of 'the design of the landscape'. The agricultural history is no longer (explicitly) visible. One could also say that this type of experience appeals to the Arcadian view of nature with its nostalgic feelings with regard to the rural life of the past, i.e. sympathy for historical elements and appreciation of the rural idyll. In the case of nostalgia the present day is compared with the past, feelings of loss or decline manifesting themselves in com- bination with a nostalgia for the past - in a sense it is a search for the safety of the past (Pickering \& Keightley, 2006). In the introduction to this chapter we stated that within the Arcadian view of nature 'weak-anthropocentric values' play a role. Anthropocentric values 'concentrated not only on the economic functions of the area, but also on the aesthetic functions of beautiful natural landscapes' (Buijs, 2009: 105). ${ }^{116}$ Arcadian thought is based on certain stereotypes, such as 'the picturesque and Romantic thropocentric values not just where attention is devoted to functional, i.e. economic function, but also where attention is devoted to aes thetic functions. landscapes', and it refers to a 'peaceful rural landscape' (Buijs, 2009: 53). The quotation of respondent 15 mentioned in $₫ 6.3$ illustrates this in a certain way.
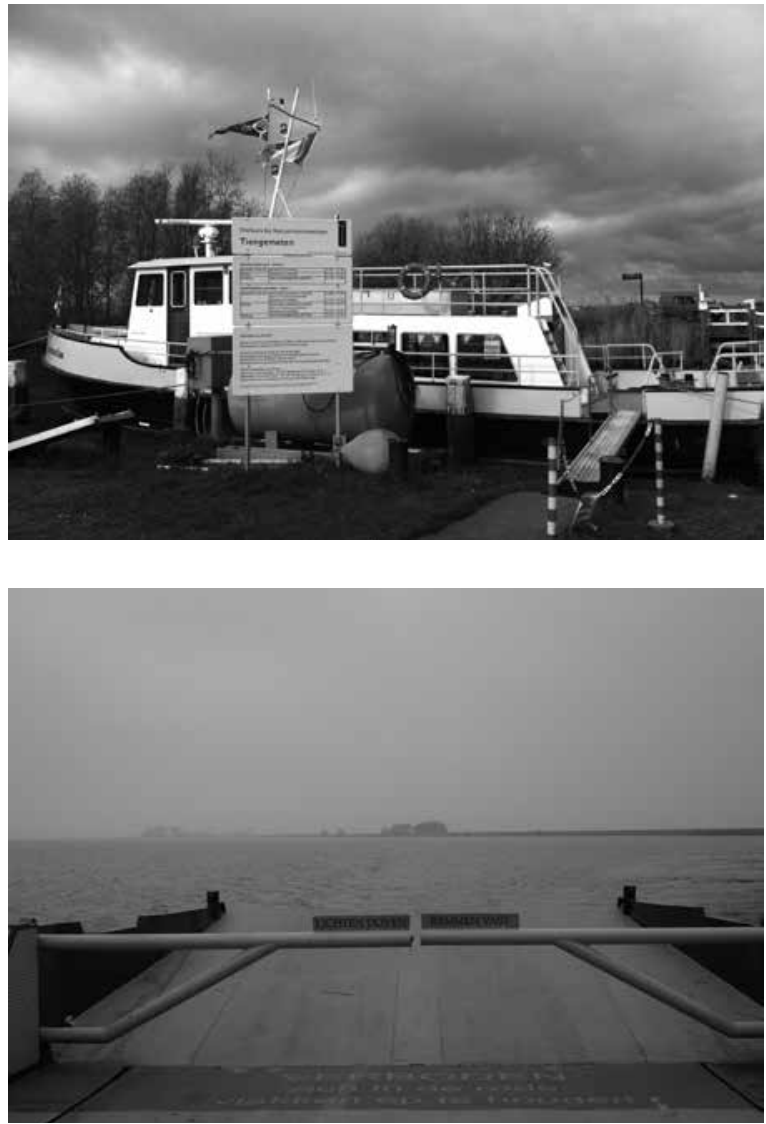

Experiences are reinforced by the required crossing by ferry (photos 5 and 6 ).

In the case of 'island feeling' the respondents mention vastness, a feeling of space, emptiness and transparency combined with a feeling of freedom and relative peace. But for this experience a counterpoint is always necessary: the mainland. Those experiences are reinforced by the required crossing by ferry (photos 5 and 
6). Words with a certain religious connotation, such as 'travel experience', 'other dimension of existence', 'reborn' and 'sort of sacred space' were used during the interviews. ${ }^{117}$ If we look at table 1 in the introduction it is difficult to classify the category 'island feeling'. This category has elements of the Arcadian view of nature - 'experiencing the beauty of nature' and 'modelled upon stereotypes of nature' - as well as elements of the wilderness view of nature, where ecocentric values - 'nature in contrast with man' - prevail.

People often go to certain places as they believe that they can find something there (Van den Born \& De Groot, 2011). This is manifest, for example, in the experience of 'escape': most visitors experience nature as a place where they can 'escape from the worries of day-to-day existence'. Nature contrasts with an environment controlled by people, which fits in with the wilderness view of nature. This is illustrated by the comment made by respondent 11 , who believes that going into nature is something of a ritual. Ritual in the sense of a repeated meaningful action, in this case to escape from the worries of day-to-day existence. Escape experiences make the third category different from the second category. 'Island feeling vs mainland', the second category, illustrates the touristic element of a visit of Tiengemeten. The word pair 'escape vs daily life' of the third category emphasizes more the aspect of peregrinatio. Interesting in this respect is the quotation below:

Pilgrimage and tourism are permeable experiences. Pilgrimage has always included 'seeing the sights' as well as worrying about finding the next bed and breakfast. And tourism frequently involves a search for personal transformation through the experiencing with others of a special site that represents central values and beliefs of the group. In both cases, the focus is on travel away from home to a special place that is set apart from daily life. (...) the expectation is that there will be some kind of transformation or re-creation as result of the visit (Ross-Bryant, 2013: 6-7).

She makes the important comment that pilgrimage and tourism are about what she calls 'transformation through experiencing', which is possible as the
117 We are talking here about comments that Voye (2004) defines as implicit religion: 'implicit religion refers to those aspects of ordinary life which seem to contain an inherently religious element within them - whether or not they are expressed in ways that are traditionally described as religious' (202).

118 Pilgrimages places are storage places for collective, i.e. cultural, ideas; she talks in this respect of 'ideals'. According to her, you can also apply this to national parks. 'A shrine, then, including a national park, provides a physica location that embodies the value that orient the culture; and travel to the location both literally and symbolically orients the pilgrim' (7) She also talks about nature as on of the symbolic centres that have oriented people.

particular place, e.g. Tiengemeten, represents certain values and beliefs. ${ }^{118}$ Some authors note that the wilderness approach builds on the idea 'that wild, uncultivated nature represents an important value that has to be protected from cultivation and appropriation by man' (Drenthen, 2003: 200). Ecocentric values are central to the wilderness view of nature.

The second part of this chapter shows that the respondents have 'feelings of being overwhelmed' in relation to nature. These experiences fit in with the wilderness view of nature. As can be seen from table 1, wilderness has a sympathy for sublime elements. ${ }^{119}$ Grand and wild nature invite unique experiences to a greater extent, as a result of which it is easier to go into raptures and to feel a moment of belonging to nature (Van den Born \& De Groot, 2011). The appealing effect of nature, about which we wrote in the introduction, is mainly visible here. The 'completely different' appeals to us in some way or other and evokes feelings of attachment and alienation at the same time. Sublime experiences are about the experience that we are part of a reality that is larger than us; we feel a distance and at the same time we feel connection. Experiences of the sublime, it could be argued, are defined as being a situation in which a person feels the tension between on the one hand being part of nature and on the other hand being outside, i.e. opposite nature.

In this third part we looked at the layers of depth, i.e. deep frames, for the four types of experience found in $\ 6$ 6.4. In other words, we noted that the various experiences can be roughly linked to the two central views of nature within the Dutch discourse: the Arcadian view of nature and the wilderness view of nature. If we place the categorisation from the second part of this chapter next to the characteristics of the Arcadian and wilderness view of nature, we can see that there

119 Willis (2011) too notes that wilderness was rediscovered as the place of the sublime. are experiences that fit more with the Arcadian view of nature and experiences that fit more with the wilderness view of nature. This is summarized in figure 5 . 


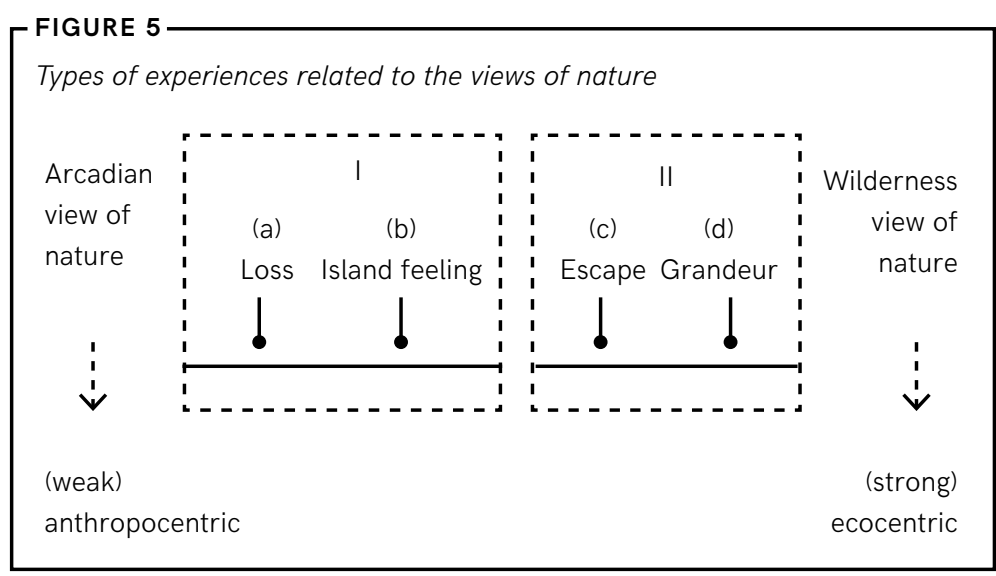

6.6 DISCUSSION

In this chapter we have tried to gain an insight in an explorative manner into what layers of depth, i.e. views of nature can be identified in the descriptions of the experience of visitors. It is important to note that somebody can have several types of experiences with regard to Tiengemeten that do not always fit with one specific view of nature. To a certain extent the respondents have a hybrid view of nature. This poses the question as to whether views of nature are manifest only on a person level or whether these views are embedded in culture. What is interesting in this respect is that 'frames seem to be everywhere, but no one knows where exactly they begin and where they end' (Van Gorp, 2007: 62). Frames exist more or less independently of the individual and are part of a culture (Van Gorp, 2007). Frames, i.e. views of nature, are 'in stock' in a culture and we use them consciously or unconsciously as we communicate about our experiences of nature. Views of nature can be regarded as stylised depictions of deep frames, which however in the case of individuals often do not occur in 'prototypical' form.

\subsubsection{World Views}

In this chapter we have adopted the distinction of layers of depth in communication. Layers of depth in communication on nature show that descriptions of experiences of nature (level of surface frames) are linked to certain views of nature (level of deep frames) that in turn embody values and convictions. This is not a static process; we should think in terms of interaction, see figure 6 .

\section{FIGURE 6}

Layers of depth in communication

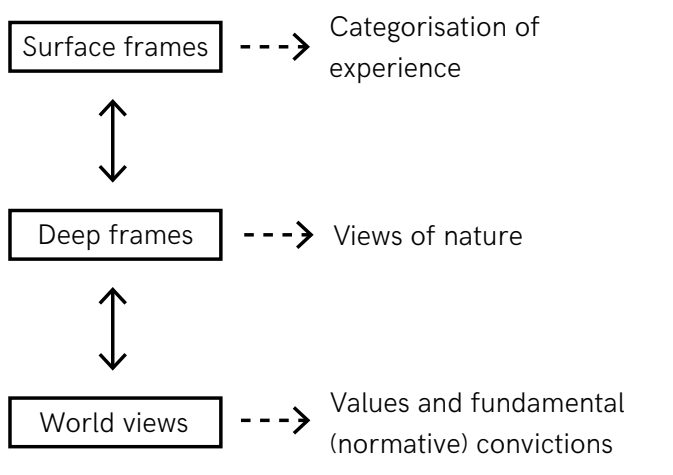

Values function as the 'guiding principles of what is moral, desirable or just' (Kempton et al., 1995: 12). Beliefs on the other hand are related with our views about and our attitudes to an object or situation, i.e. a value perspective. This is often translated into a certain fundamental attitude. ${ }^{120}$ In other words, behind views of nature world views once again appear. World views are contextual phenomena that allow people to see things in a certain way and enable people to make connections between things. They define a person and provide people with 'fundamental assumptions upon which a life is based' (Naugle, 2002: 291).

\subsubsection{Notions of the Sublime}

It can be stated in general that in the experience described by visitors the wilderness view of nature is particularly evident. The description includes sympathy for sublime elements: wind, clouds, etc. Especially the unpredictable character of nature and nature's own course are stressed. The descriptions also include certain stereotypes of 'real' nature. For example that 'real' nature develops autonomously without (too much) involvement by people. It is interesting that some authors argue that sublimity implies a connection between God and the wilderness (Nash, [1969] 2001). Or that by a sublime experience 'certain 120 Various classifications have landscapes came to be valued as holding the promise been developed, but that of Zweers of revealing the face of God' (Willis, 2011: 95). The (1989) is the best-known within the the Dutch discourse. He distinguishes six fundamental attitudes to riences is in line with the words of Otto ([1917] 1923) nature, positioned on the line an- about experiences of the numinous - a mood that thropocentric - ecocentric: despot, ner, participant and 'unio mystica'. 


\section{TEXTBOX 2: Numinous experiences}

We use 'holy' generally as the absolutely moral predicate, as 'completely good' (Otto, [1917] 1923: 5). But as our feeling for language 'undoubtedly always feel the morally good to be implied in holy' it is recommended, according to him, to find a special term 'to stand for the holy minus its moral factor (...) minus its rational aspect altogether' (6). Otto ([1917] 1923) continues: ' (...) I adopt a word coined from the Latin numen. Omen has given us ominous, and there is no reason why from numen we should not similarly form a word "numinous". I shall speak then of a unique numinous category of value and of a definitely numinous state of mind, which is always found wherever the category is applied' (7). It is a category that, according to him, is individual and, just like any other original fact, cannot be accurately defined - the numinous is only demonstrable. ${ }^{121}$

However, it is striking that the visitors in general talk about their experiences with nature in a fairly 'flat' or superficial way. Is it diffidence or is an interview setting 'too threatening' to be more personal? Are we lacking, in the Dutch 'neutralised public discourse', language that is connected to meaningful sources that offer us possibilities for describing and defining spiritual experiences? In other words, is there no (longer any) cultural substrate for talking about such experiences? However, it may also be that this 'flat' way of speaking is not uncommon for human experiences of the numinous. After all, these are experiences that are demonstrable but difficult to articulate.

Although the focus of this chapter is on visitors, it is interesting to note that the key actors talk more easily about this category. Or put another way: key actors seem to more easily interpret their experiences of nature as spiritual or religious. Several key actors talk, for example, about a meeting with the Greater Whole in nature or about nature as a source of meaning. ${ }^{122}$ One key figure even said that nature is a place to return to be reborn. ened' (7)

\subsubsection{Green Religion}

Table 2 seem to indicate that the difference between visitors and key actors, as described in the previous section, can be partly explained by the fact that $80 \%$ of the key actors are to a greater or lesser extent 'involved with religion' compared with approximately $47 \%$ of the visitors.

TABLE 2 Religious background respondents

\begin{tabular}{|l|r|r|r|}
\hline Type of religion & \multicolumn{1}{|c|}{ Visitors } & \multicolumn{1}{c|}{$\begin{array}{c}\text { Key } \\
\text { actors }\end{array}$} & \multicolumn{1}{c|}{ Total } \\
\cline { 2 - 4 } & 15 & 10 & 25 \\
\hline Christian & $33,33 \%$ & $20,00 \%$ & $26,67 \%$ \\
\hline Previously Christian & $0,00 \%$ & $50,00 \%$ & $25,00 \%$ \\
\hline $\begin{array}{l}\text { Spiritual, never involved in an institu- } \\
\text { tional form of religion, believes in a } \\
\text { Higher Power, regardless of what it is }\end{array}$ & $13,33 \%$ & $10,00 \%$ & $11,67 \%$ \\
\hline Not religious or (consciously) spiritual & $53,33 \%$ & $20,00 \%$ & $36,67 \%$ \\
\hline
\end{tabular}
fore argues that you can only generate understanding with the listener by trying to help them using a detailed discussion so that the category comes to life in their own inner lives, escapes and they become aware through it. You can support this method by pointing out the opposite that is similar or also characteristic, which occurs in other already known, familiar at mospheres, and then adding: 'this $X$ of ours is not precisely this experience, but akin to this one and the opposite of that other. Cannot you now realize for yourself what it is? (7). That means: strictly speaking you cannot state what our $x$ consists of, you can only incite and encourage another person to discover it themselves - 'as everything that comes of the spirit must be awak

122 Respondent 16 states, for expes for me nature is an for portant source of meaning, but only by being able to bond with nature, by being able to be a part of it'.

The results of a study done in the US by Ferguson \& Tamburello (2015), and a statement of Taylor (2012) are interesting. Ferguson \& Tamburello (2015) conclude that the natural environment offers a spiritual substitute for traditional religious groups, but also that the natural environment 'may be a supplier of spiritual goods to the religious unaffiliated, or 'nones'
123 They define religious 'nones' as 'people who do not identify with any religious tradition', but 'this does not mean that this group is irreligious'

124 He notes that it is a matter of perception and definition whether some of these nature-based spiritualties count as religion. However, ssume

beings there is no religion, for there are many examples around the world where people feel and speak of a "spiritual connection" to nature, or of "belonging to" the earth (mother arth, or even mother ocean), or speak of the earth as "sacred" without any concomitant confession of supernatural beings' $(1000)$ (5). ${ }^{123}$ Taylor (2012) observes that the efforts to establish and protect wilderness areas can be understood 'to be a way of establishing such places as sacred places, as temples for those who have left behind conventional religions' (310). In an earlier publication he reported an increasing interest in what he calls 'nature-based piritualities', i.e. 'green religion' (Taylor, 2004)..$^{124}$

Environmental degradation has become increasingly obvious and alarming, and this is increasingly grafted into existing religions (green religion type 1), and mixed in with revitalized and new forms of This represents a significant innovation in the history of religions, where apocalyptic expectation arises nature-as-sacred religions (green religion type 2). 
not from the fear of angry divinities or incomprehensible natural disasters but from environmental science. (...) It may be that the third type, now only nascent and growing within small enclaves of devotees around the world, will inherit much of the religious future; a religion in which people feel awe and reverence toward the earth's living systems and even feel themselves as connected and belonging to these systems (Taylor, 2004: 1001-1002).

It is (green) religion of the third type that is particularly interesting within the framework of this chapter. In another study, Taylor (2001b) talks of 'earth-based spirituality'.

Earth-based spiritualties are based on personal experiences that foster a bonding with nature. These experiences are diverse, take place in different venues and are expressed in plural ways that are sometimes contested (Taylor, 2001b:226). ${ }^{125}$

Despite the differences there is a common 'faith'.

It can be stated succinctly: the earth and all its life forms and processes are sacred. We belong to them and they to us - we are kin. We should, therefore, act lovingly, reverently, and respectfully toward them. We must not unnecessarily injure these beings and processes when we take from them what we need to live (Taylor, 2001b:238). ${ }^{126}$

How does this connect to the themes of this chapter? If in the Netherlands the reclamation of land is linked with Christianisation, it could be argued
125 He continues by stating that 'these feeling may be intentionally precipitated through a variety of practices that are themselves a creative bricolage assembled from older religious traditions, especially Eastern and indigenous religions, combined with new inventions, such as neo-paganism, all stirred into an eclectic spiritual stew' (226).

126 But regardless of this convergence accord ing him tensions will still remain. 'Devotees of the more mystical and super naturalistic forms of nature-based spirituality, those with a more cosmological or astrological referent, or those that tend to personify Gaia as goddess more than as an ecological process are more likely to be optimistic about humans and their ability to manipulate nature's energies, mentally or technologically, in a beneficent manner (...) Those engaged in the most naturalistic forms of nature-based spirituality tend to take the earth and its biological processes as the axis of their worldview of nature. They are likely to discuss Gaia more as a living, biological system than as a god dess. (...) But the key is a felt sense of "connection", kinship and loyalty to earth and all her life forms and living systems' (Taylor, 2001b:238, 241).

127 In the past the Dutch have wrested land from the sea, i.e. turned the swampy and unstable river delta into a country with a strong agricultural identity. This process is known as inpoldering. Nowadays, for nature development reasons a lot of farmland is to be flooded again - this process is called depoldering, so it means 'giving land back to the water'.

128 According to Casanova (2006) in genera secularisation has three different connotations: (1) secularisation as the decline of religious beliefs and practices in modern societies, (2) secularisation as the privatisation of religion and (3) secularisation as the differentiation of the secular spheres, i.e. emancipation from religious institutions and norms. that conversely there is a link between depoldering and secularisation (Zwart, 2011). ${ }^{127}$ However, secularisation does not by definition mean a reduction in individual religiosity. ${ }^{128}$ Authors such as Stolow (2005) note, for example, that there is a religious revival going on in what he calls secular modernity. Religion did not disappear, but took a different shape and form. We no longer have secularisation as the standard intrinsic to modernity, but we are more 'alert instead to the specific ways in which the concept, role and place of religion have been redefined with the rise of secularity' (Meyer, 2002: 6). Or more specifically with regard to this chapter: is there a link between the rise of the wilderness view of nature and the increase in 'nature-based spiritualities', and can Tiengemeten be seen as an example of the transition of religiosity presenty taking place in our Western society?

Our study shows so called 'notions of the sublime' in the experiences of visitors. It turns out that 'when a person hikes in a forest to connect with the sacred, she or he may not feel the need to affiliate with a religious organization because her of

his spiritual demands are met' (Ferguson \& Tamburel129 They note that the natural lo, 2015: 5). ${ }^{129}$ Ferguson \& Tamburello (2015) further environment differs from other traditional suppliers of spirituality, because it 'do not have the ability to place constraints on individuals to prevent them from frequenting other suppliers, as religious organizations are often able to do through their cultures' (...) 'many religious congregations also meet during specific hours each week, particularly during the weekend. Natural

resources, however, do not have If the natural environment were a spiritual resource, these time uals may use them whenever they please. Therefore, natural amenities are not really competitors with traditional religious organizations for the population's time. Instead, because they have the ability to satisfy a portion of the population's desire for spiritual fulfilment, they compete for allegiance' (5). Or as they note later on in their article:

the evidence supports our hypoth- With this in mind, it is not too far-fetched to postulate esis that beautiful regions have lower rates of membership and affiliation, not just lower rates of religious behavior' (15). argue that 'scholars have noted that nature-based

pirituality may be drawing unaffliated individuals way from traditional institutions' (Ferguson \& Tamburello, 2015: 5). Although not all nature experiences re competitive with religious adherence, we can

conclude that there is a certain relation between both, as the quote below also shows. it would necessarily compete within the religio-spiritual marketplace with other, more tradition al religious resources.(...) Many people [in the United States, PJ] meet their spiritual needs through their ocal religious congregation, and so naturel amenities may be competing with congregations as a supplier or spirituality (Ferguson \& Tamburello, 2015: 4-5). that the rise of the wilderness view of nature results in an increase of 'nature-based spirituality' and decrease of religious adherence. This conclusion opens up 
possibilities for future studies. It would be interesting to explore in greater detail the relationship between the rise of a wilderness view of nature and religious adherence in the Netherlands.

To conclude, our study extends research on nature experiences. Our findings help to get more insight into visitors' experiences of nature development projects like Tiengemeten. Our study also gives insight into the role of 'place' in nature experiences, especially regarding experiences of loss and island feelings. Finally our study expands understandings of religion and spirituality. It may be contribute to a better understanding of the field of nature

130 Taylor (2001a) notes that flexible definition of religion provides a good starting point for the present endeavour to understand experiences in relation to religion and spirituality. ${ }^{130} \quad \begin{aligned} & \text { earth-based religion and spirituality } \\ & \text {. }\end{aligned}$

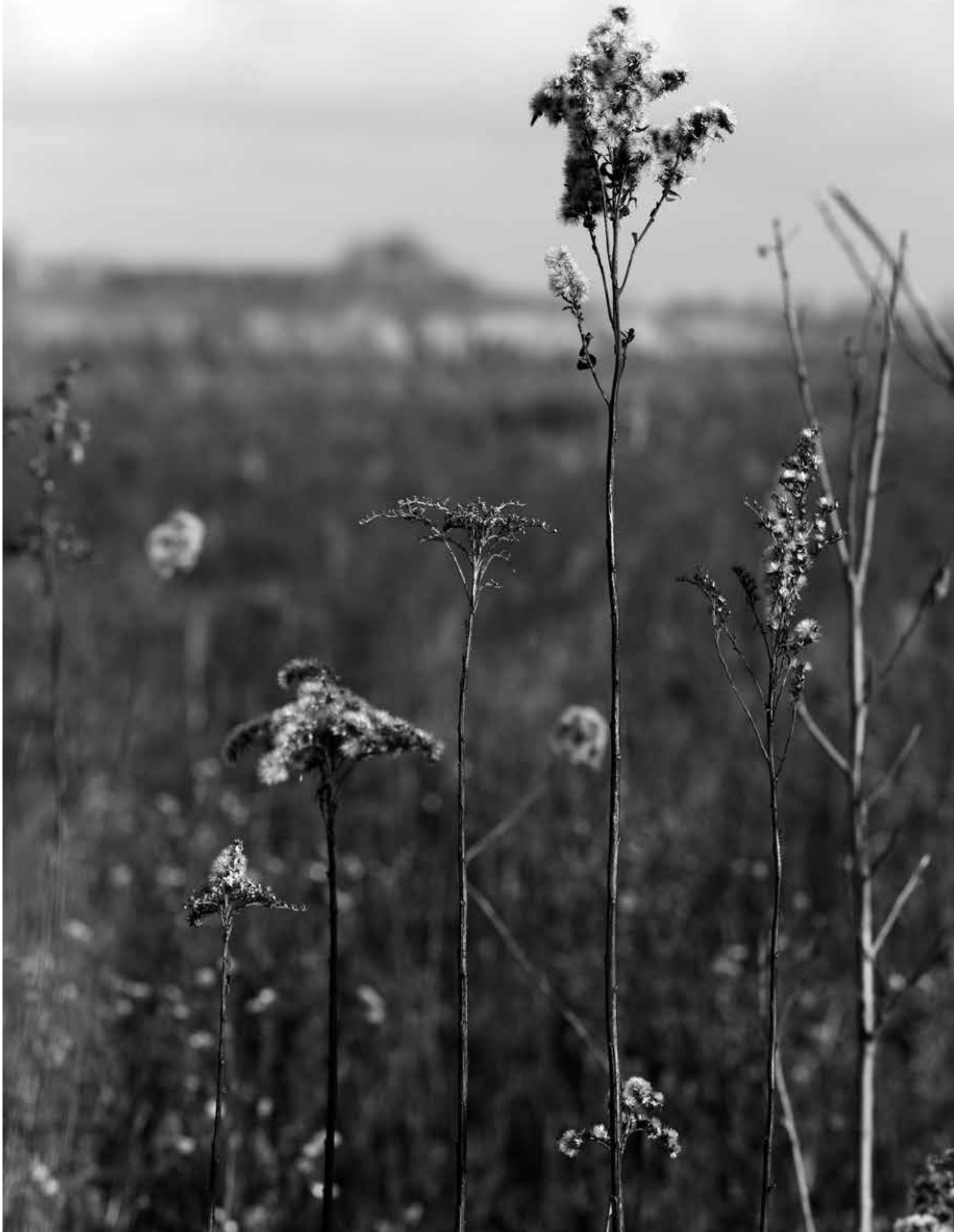




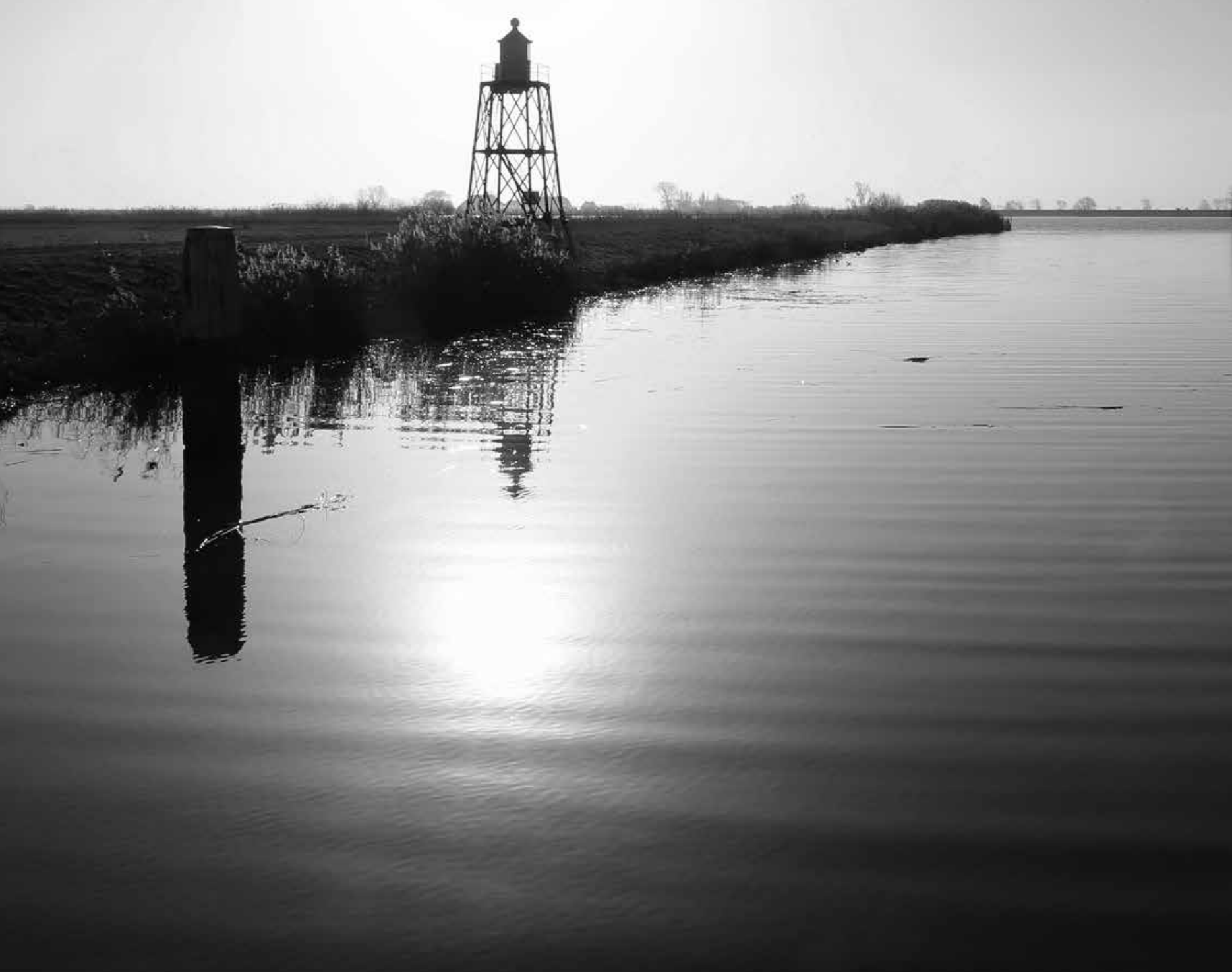

General

Discussion 


\subsection{EVALUATIVE AND METHODOLOGICAL REFLECTION}

In addition to a general introduction (chapter 1) and a general discussion (chapter 7), this $\mathrm{PhD}$ thesis consists of five sub-studies (chapters $2-6$ ). Each chapter contains a new topic related to the general theme of this $\mathrm{PhD}$ thesis. As a matter of fact, each chapter is an almost independent study; each has been published (chapters 4, 5, and 6) or accepted (chapters 2 and 3 ) as a peer-review article in a scientific journal. Chapters 2 and 3 are conceptual and contain, on the basis of a study of the literature, a description of the changing context of both the nature policy and government communication. This changing context has an influence on the relationship between the government and its stakeholders. I argue that this calls for rethinking (1) the question as to what government communication is and (2) the role of government communication practitioners who work in this changing context. Chapter 2 discusses the first question in a conceptual analysis of government communication; chapter 3 answers the second question by regarding government communication as a practice. This third chapter is in keeping with the normative practice model, usually abbreviated to NPM, as developed within reformational philosophy (chapter 1). The most significant advantage of the NPM is that it shows that the aim of a practice is, first and foremost, the pursuit of the 'good' of the practice rather than (striving for) success. As argued in chapter 3 , all things in which the 'good' of the practice is sacrificed for (personal) success violate that practice. It is recommended that the conceptual points from the third chapter be discussed with representatives from the field of government communication considering several practical cases so that the model can be refined and optimised.

The fourth chapter is intended as a theoretical prelude for the more empirical fifth and sixth chapters of this PhD thesis. It is based, on the one hand, on a study of the literature and, on the other hand, on mainly the communication of NGOs which, in the context of this $\mathrm{PhD}$ thesis, function as delegated executors of the Dutch nature policy (chapter 1). In chapters 5 and 6 , the focus is on a qualitative case study in the form of semi-structured interviews with visitors and key actors in policy and communication. In a case study, generalisation is not the primary aim (cf. Gibbs, 2007; Saunders et al.,2011). This is because it is more often about testing a certain hypothesis or theory than about evidence in the strict sense of the word (cf. Smaling, 2009; Plooij, 2011). Yin (2009), therefore, chooses to refer to an experiment rather than a research unit in relation to a case study: 'case studies, like experiments, are generalizable to theoretical propositions and not to populations or universes' (15). Marshall \& Rossman (1999) state that, if the case can be linked to important theories, this shows that the results are of wider significance than just the case that is used for the research.

In regard to the methodology, I adopted that of Baarda et al. (2009; cf. FootNote 98) who list three (evaluation) criteria with regard to qualitative research: geloofwaardigheid (credibility), transparantie (transparency), and adequaatheid (appropriateness). In my own words: (1) was the research conducted in a convincing manner, (2) is the research traceable, and (3) is it appropriate to the situation. ${ }^{131}$ I shall reflect briefly on these three questions.

\section{CREDIBILITY}

As a component of the case study, 25 people were interviewed: 15 visitors and 10 key actors. As described in the methodology section in chapter 5 , data saturation is the guiding principle in interviewing (Mason, 2010). In our case, saturation was achieved with approximately $12-15$ (visitors) and $8-10$ (key actors), that is, 20-25 interviewees in total. This is in accordance with the 'norms' relating to data saturation in qualitative research (see FootNote 99). However, the research would have been more valuable if it would have had a more longitudinal character and if a comparative study had been performed at both a national and international level. This research signals trends; however, longitudinal and comparative research could indicate how substantial these trends are. The international comparative aspect is particularly valuable in this respect. This can make it possible to take into account, to a greater degree, the cultural character of views of nature and the influence of a certain location on experiences in relation to a shift in religiousness. These are possibilities for follow-up research.

\section{TRANSPARENCY}

Regarding the traceability of the research, it can be noted that the methodological section of chapter 5 and Textbox 1 of chapter 6 have been primarily written to ensure the traceability of the empirical part of my research. In addition to regular consultations with fellow researchers, the various 131 Cf. Smaling (2011). He re stages of the research were made transparent and he believes go beyond the empiri- justifiable, i.e., (partial) results of this research were cal-analytical approach, for exam- presented and discussed at various international sciple coherence, intersubjectivity, entific conferences. ${ }^{132}$ These meetings also influenced 132 These were scientific confer- the validation of the research: determining the focus ences both in the Netherlands (2x) of the several studies, advice regarding the research and abroad, i.e., Belgium (1x), Esto- approach, reflection on preliminary results, etc. States of America (3x). 


\section{APPROPRIATENESS}

The focus of this research was an assessment of whether religious elements in the communication of nature can be identified. As described in theory in chapter 4 and worked out empirically in chapters 5 and 6 , this is closely related to experiences in nature. A hermeneutic approach, as described in the introduction (chapter 1) and a qualitative case study seem to be the most obvious methods for such a topic. Experiences are difficult to express in terms of quantitative data, therefore, a more hermeneutic approach is the most suitable. Meetings with peers also indicated that the selected methodology was determined as being appropriate for the set goal, which is also shown by the acceptance and publication of all of the studies in scientific journals. ${ }^{133}$ However, the subject matter of this $\mathrm{PhD}$ thesis would be enriched if, in addition to interviews, additional detailed text analysis were performed on nature policy documents or government communications in the broadest sense of the word. Follow-up research in this field is recommended. The aspect of 'secular spirituality' in relationship to nature visits and experiences, as covered in $\$ 7.6$, provides starting points for further exploration.

\subsection{NORMATIVE CHARACTER OF COMMUNICATION}

I will now discuss the first of the four themes under which I will explore the main results of this $\mathrm{PhD}$ thesis. One of the conclusions of this thesis is that there is a change in the relationship between the government and its stakeholders (chapter 2). To an increasing extent, the government negotiates, whether or not voluntarily, with citizens and interest groups. In this negotiation, each participant has his/ her own substantive role and his/her own interests. Awareness of these interests is important as it provides insight into the so called justified interests (Verkerk, 2014). However, it also assists government communication practitioners in finding a connection with the wishes and interests of citizens and other stakeholders (Buijs, 2009). However, this poses the question as to the extent to which the government must be explicit about its own attitudes and its normative convictions, whether these are consciously communicated or not. In this context, this $\mathrm{PhD}$ thesis also shows that words and images used in a discourse are not separate concepts or phrases; they entail a web of meanings and are rooted in convictions (chapter 2). This brings us to the topic of frames.

Inspired by Lakoff (2006), we have made a distinction between surface frames and deep frames (chapters 2, 4, and 6). Certain words have a specific
133 In addition to FootNote 132 the chosen methodology was part of a multi-day PhD seminar, or ganised by the European Public Relations Education and Research Association (EUPRERA) and hosted by the Faculty of Communication at Istanbul University (Turkey). meaning in our daily language usage. The word 'wilderness', for example, is often associated in daily language usage with 'rugged nature'. Surface frames clarify what is occurring and provide us with a certain mind set. However, there are also deep frames. These are more difficult to identify empirically, but they are crucial for understanding communication. Deep frames articulate our more fundamental convictions that shape our perception and assessment of reality. To use the example above, wilderness as a deep frame has the meaning of 'true nature', 'nature as it was originally', etc. Deep frames, therefore, have a close relationship with our world view. Wilderness, for example, is in keeping with an ecocentric, objectivist world view (chapter 4)

Normative elements can be discovered both in the various interests and in the associated (deep) frames. Although Fischer (2007) primarily focused on policy analysis, his concept of 'argumentative turn' sounds interesting in this respect (chapter 2). The concept of 'argumentative turn' begins with the recognition that 'multiple perspectives are involved in the interpretation and understanding of social and political reality and the competing definitions of policy problems to which they give rise' (224). In other words, various perspectives - or 'underlying assumptions', as Fischer (2007) also terms them - play a role in a debate, and these various perspectives, each with their own norms, also need to be recognised. In fact, the concept of 'argumentative turn' shows that decision-making begins with making the perspectives and norms explicit to which the participants in the controversy are committed (chapter 2). For that reason, Fisher (2007) approaches a policy debate, for example, as an organised dialogue among competing normative positions. In such a policy debate, he argues, each party would confront the others with counterproposals based on varying perceptions of the facts. Otherwise stated and more in accordance with the terminology of this $\mathrm{PhD}$ thesis, a debate is about the world view based interpretations of observations.

If Fischer (2007) is correct, this is consequential for the role of government communication practitioners. Their task is not to primarily produce communication materials or transmit content; more than ever, communication practitioner are 'spiders in the web' of normative convictions. This $\mathrm{PhD}$ thesis presents the opinion that the role of government communication practitioners is to signal, articulate, and interpret these normative elements in the discourse. Or, in other words, to make frames explicit, be they non-congruent or not, and to make associated world views transparent in the discourse, including that of the government itself. In its communication, the government must be as impartial as possible (cf. Klop, 1996). However, the communication regarding nature demonstrates that 
the neutrality of government communication may be questioned. Certain images about nature, i.e., frames, are communicated - not directly by the government but through the communication by NGOs that act as delegated executors of the Dutch nature policy (chapter 1), or more precisely, it is striking that there are 'religious subtexts' in the communication of the NGOs involved (chapter 4 and \$7.4). This poses the question as to the extent to which openness about this is important in relationship to social and political legitimacy.

Chapter 3 argues that social legitimacy is an intended effect of government communication. This means that, initially, this is about adequate communication of the how and the why of the actions of the government. ${ }^{134}$ Otherwise stated, the usefulness and the necessity of certain actions need to be made explicit and to be convincingly and truthfully communicated. Convincing and truthful communication implies, as is argued in chapter 3 , a positive response to (i) the question as to whether the communication is consistent with the 'telos' of the government, ${ }^{135}$ (ii) the question as to whether the communication is in accordance with how they actually act, and (iii) the question as to whether it is in accordance with how they communicated earlier. A negative response to one of these three questions violates reliability and thus social legitimacy. Therefore, it is not a fixed story - citizens and stakeholders will see through this - but a sound story (Van der Stoep, 2016). This implies that government communication is a question of not just factuality but also of normativity. Stories generate trust only if they are sound. This means that sound communication, i.e., convincing and truthful communication, is a condition for social legitimacy. ${ }^{130}$

\subsection{RELIGIOUS SUBTEXTS IN}

\section{THE COMMUNICATION}

This $\mathrm{PhD}$ thesis concludes that there has been a change regarding the Dutch nature policy (chapters 1 and 4), i.e., a shift from a focus on retaining and conserving existing nature to the development of so-called 'new nature'. Renewed attention to
134 For this reason, government communication is regarded in chapter 3 of this PhD thesis as a linguistic activity in which the social aspect is supplemental, in which 'conveying meaning' can be defined as the 'telos' of the linguistic aspect. Otherwise stated, in this PhD thesis, government communication is regarded as being a 'linguistic interaction' with the aim to share meanings. 135 In chapter 3, it is argue that the 'telos' of the government is 'public justice'. This assumes that the government takes into ac count the situation and the parties involved, i.e., that the so-called 'justified interests' are handled in a convincing and truthful manner.

136 It is important that this be distinguished from the question of whether all of the stakeholder agree with the content of this. If communication is sound, it can be assumed that the stakeholders experience the government's input as a legitimate input and accept any decision, even if they do not agree with it. Democracy is not so much about citizens agreeing with the actual policy of the government and government's actions but that the can trust the preparatory democratic decision-making. wilderness is connected to this 'new nature'. However, strictly speaking, there is no true wilderness remaining in the Netherlands. Consequently, the Dutch have sought to create new wildernesses through the concept of nature development. In other words, the Dutch have attributed values such as 'real' or 'pure' to cultura landscapes in order to find some 'wilderness nature' in their country (chapter 4). In this respect, the vision presented by the Dutch government in 2014 is important. The document claims not only that the distinction between 'true' nature and 'other' nature is outdated but also, and above all, that citizens require nature to 'recuperate' and that they seek 'excitement and wonder' in nature. This mean that nature development is no longer driven only by the ideal to restore 'pure nature' for the sake of nature herself but also by the value that such nature has for human experience. In a sense, the way in which nature has been constructed and managed in the Netherlands is driven by a perceived desire of citizens to experience nature (chapter 5)

This poses the question of where this 'longing for wilderness' comes from. Underlying the call for wilderness is an increased sense of loss of contact with nature (chapter 4). We can also see this if we examine the communication of NGOs. An appeal is made, above all else, for 'an escape from daily life'. Nature is presented as the 'better world' and, in the communication, a type of promise is made: you can find yourself again in nature. This is in keeping with what we need, according to the vision quoted above and presented in 2014. Boomars (2001) states on the basis of empirical research into communications about the Veluwe, for example:

Creating an atmospheric picture of the Veluwe and expressing this modality in repetitive, clichéd terms connects to the yearning for peace and space in order to refuel and find yourself (Boomars, 2001:52).

What this shows is how words and images contribute to the 'sacralisation of nature'. Nature does not determine its position itself nor can it attribute the qualitie referred to itself (cf. Schama, 1998). We attribute these meanings to it, not at the least through our communication. The meanings that we attribute to nature are fairly idealistic and the communication about nature contains, on closer inspection, so-called 'religious subtexts' (chapter 4). However, it is striking that these 'religious subtexts' are not necessarily of a theological nature. More precisely, the examples suggest, rather, that nature is valorised and 'religionised' without any mention of a personal God or other supernatural agents (chapters 4, 5, and 6). This might probably be part of the success of this discourse in a decreasingly Christian country. 
However, the situation above poses the question of the extent to which the government should condition the communication of the NGOs from its position of supposed neutrality. Do 'religious subtexts' belong to communication about the effects of the government policy? As we have seen in the introduction (chapter 1), the communication about nature projects such as the Tiengemeten is actually performed by a nature conservation NGO, i.c., Natuurmonumenten. However, this is done in close cooperation with the government and within the contours of the governmental policy in such a way that such communication can be considered as warranted by the government. In discussing the findings with respect to the 'religious subtexts', a distinction should be made between the government and nature conservation NGOs. In terms of reformational philosophy, the government is juridically qualified, whereas the NGO is morally qualified (chapters 1 and 3). This qualification has implications for their communication. In contrast to the government, nature conservation NGOs as such are not bound by so called 'justified interests' of different stakeholders (chapter 3 and $\$ 7.3$ ). More strictly formulated, the reason to exist ('raison d'être') of nature conservation NGOs lies in their (moral) value-orientation and the view of (an element of) the 'good life' behind that. This binds people to them, and it also legitimizes their actions ('license to operate'). Yet, in the case of Tiengemeten and other nature conservation/ development projects, NGOs are de facto executors of the Dutch nature policy (chapter 1). Here, tension can be observed. If nature conservation NGOs (explicitly) communicate a specific vision about nature using 'religious subtexts', the government appears to support these 'subtexts'. For nature conservation NGOs, it is appropriate to put forth a certain opinion to raise support for their actions among the public. However, the government is juridically qualified with public justice as the core value. Even though there is a normative view of the 'good communal life' behind any conception of public justice, in reformational philosophy, it is not the responsibility of the government to promote a specific religiously phrased view of nature and nature policy. In doing so, the government uses its power for inappropriate purposes. I agree with Klop (1996) where he observes that 'we must avoid a situation in which another aspect (other than the qualifying) becomes predominant. Then the structural principle of the existing societal institutions will be lost' (318). Hence, this $\mathrm{PhD}$ thesis reveals a necessity for reflection on the relationship between government and NGOs regarding their communication, i.e., awareness of distinction and a need for mutual adjustment in the case of close cooperation.

\subsection{PARADOX IN NATURE EXPERIENCES}

Attitudes toward wilderness have changed in the Netherlands (chapters 4 and 5). Previously, the ideal of unspoiled wilderness was projected onto or recognised in existing nature reserves that were protected from human influence. Currently, the desire for wilderness serves as justification for human interventions in the context of the creation of 'new' nature, of which the national nature network in general and the transformation of Tiengemeten in particular are illustrations. It is striking that, in this aspect, we find a certain functional way of thinking. That is why this $\mathrm{PhD}$ thesis refers to a 'new nature paradox': nature development is linked to current ideas on technology while attempts are made to achieve a prehistoric type of nature independent of humankind (chapters 4 and 5). We also see this among the respondents: most of the key actors associated nature with something that involves a minimal amount of human interference

137 Negative feelings about the transformation of Tiengemeten from an agricultural to a nature island are generated mainly by the loss of identity of the area and the visibility of the (agricultural) hisorical origins. The identity of a certain spot combined with the interweaving of personal life histories produces feelings of connectedness with the landscape (Lemaire, 2011; Van den Born \& De Groot, 2011). This is an aspect that seems to be forgotten in nature development projects; this also came up in the transformation of Tiengemeten as evidenced by the meetings with the respondents. However, research by Van den Born et al. (2011) also shows that residents and tourists find the new landscape that emerges as a result of nature development attractive in a certain sense but do not feel connected to it as much.

138 There is a consensus among the respondents that wilderness can be regarded as nature in its 'purest' form. This result does not differ from those of earlier esearch (cf. De Groot \& Van den Born, 2003; Van den Born, 2008; Buijs et al., 2009). the involves a minima anount of hum interference human interference (chapter 5). At the same time, there is awareness among all of the respondents - i.e. here is no demonstrable difference between visitors and key actors - that nature in the Netherlands has een influenced by humans or consciously developed. In other words, among the respondents, there a hybridisation of wilderness nature and Arcadian nature, of appreciation of 'new nature' in combination with melancholic trips to the past. ${ }^{137}$ This means that a certain restraint can be perceived among the respondents in the use of 'true' nature. The use of this type of erminology implies that there is also such a thing as 'untrue' nature. Among the respondents, 'true' nature as more the connotation of spontaneous and autonomous development and a lot less pure or original. ${ }^{138}$ In other words, the results of this $\mathrm{PhD}$ thesis suggest that the nature-culture controversy is not so much a dichotomy of 'true' versus 'untrue' nature but should maybe be regarded more as a continuum that runs from spontaneous/autonomous to domesticated. ${ }^{139}$ Interesting in this respect is an interview in National Geographic (August 2016) with Martin Drenthen where he indicates that he prefers the concept of wildness rather than wilderness. The concept of wildness, 
according to him, is not untouched nature but a certain quality that has to do with what lies outside people that we cannot control or determine; according to him, it has its own vitality and spontaneity that surprise us. ${ }^{140}$

The case study of Tiengemeten, as described in chapters 5 and 6 , also unearths another paradox: creating nature 'according to our view of nature' and, simultaneously, wanting to experience wilderness-nature. This paradox appears to be a cultural foundation to (new) nature development projects. This poses the question of whether the current nature policy with its emphasis on nature development and on nature experience can be understood without taking the cultural context into account. Van der Woud (2007) notes the following with regard to Tiengemeten:

We cannot escape the fact that this new nature has an important aesthetic dimension. It gives the impression of having a prehistoric or early-medieval antiquity - a landscape that existed before people began to tinker with it and to enslave nature. (...) Leaflets and information signs inform people about the rich biotope - if this cannot yet be fully seen, then have some patience. (...) This postmodern artificial type of landscape with its ancient evocation has striking similarities with an English garden. The island of Tiengemeten, which measures a thousand hectares and is located in the Haringvliet, was agricultural land when Natuurmonumenten bought it in 1997. It has recently been 'reconstructed'. The brown, long-haired ancient cattle, bred specially for the new landscapes, wade through the carefully ruined, submerged fields. This is the new park for the south wing of the Randstad. People (essential for support and money) can walk and look around at planned 'natural' spots. The merchandising of this primitive, unspoilt nature requires well-dosed 'experiences'. These are deliberately staged in order to provide the spectators with nice images of the past: a haystack, an old boundary post, a
139 Cf. Van den Horn \& Hausdoerffer (2017) conclude that you can determine two positions about nature in contemporary discussions: first, that nature is at its wildest in the absence of human presence and, secondly, that nature has been completely humanised, and nothin is truly wild anymore. In their book, they opt for the concept in which nature exists in variations and degrees that range, as they mentio on the cover of their book, from cultivated soils to multigenerational forests to sunflowers pushine through cracks in a city alley.

$140 \mathrm{He}$, therefore, concludes that wildness is about what philosophers call the transcendent, that which transcends people. According to him, we can have such experiences in many places, including in cultural landscapes. Somethin happens that overwhelms us and therefore, is given a special signifcance. 'If we see a deer when we'r walking in the wo us. (...) If we knew in advance that the deer would be there, the en counter would have a completely different significance. ragged field with an old-fashioned crop. They act as the follies in an English garden (Van der Woud, 2007:11-13).

It is the link with the English garden that is intriguing. Van der Woud (2007) writes that the English garden 'rejected everything that was cultivated in the French baroque garden and created a beauty that was based on opposite starting points' (5). He continues:

The English garden, in which nature developed freely, emerged in contrast to the Baroque concept, in which nature was subjected to a rationalist idea. Both were really artificial artefacts - however, the romantic English landscape style conquered Europe as it denied the artificial so well that it appeared 'natural' (Van der Woud, 2007:5).

Can we draw the conclusion, applied to the theme of the (new) nature development, that projects such as Tiengemeten can be regarded as a reaction, in this case, a countermovement to the functional and rational ideas of modern time? The reactions of many respondents point in this direction: they visit Tiengemeten to find rest, find themselves, inject some balance into their life, etc. (chapter 6) which is an outcome that accords with the conclusions of Hartig et al. (2014). They conclude that contact with nature has a positive mental effect, for example, mood improvement, stress reduction, etc. This is again in keeping with the direction taken by the government's vision paper referred to above. Citizens need nature in order to 'recuperate' and do not want to just conserve nature 'but also to experience and use it' (Ministry of Economic Affairs, 2014:16). This is characterized by a certain form of anthropocentric thought about nature: we generate the desired experience, i.e., we create (new) nature as we need it. This not primarily a negative comment; it opens up new perspectives regarding nature policy and nature development. It is interesting in this respect, for example, how national parks are regarded in the American context according to Ross-Bryant (2013).

We will look at the national parks as sacred places - spaces set apart (...) places where the normal boundaries are lifted (and) where people can explore nature outside the parameters of culture sets and play with conflicting ideals of the culture (Ross-Bryant, 2013:4).

The above quote makes it clear that (new) nature projects are regarded as valuable not only from an ecological point of view. Experiences can be had that help us better understand our existence in this world (cf. Appolloni \& Hrynkow, 2016). 
In terms of this $\mathrm{PhD}$ thesis: they are places to gain meaningful experiences. This makes (new) nature projects, such as Tiengemeten, valuable from a societal point of view as well as an ecological one. Including this perspective in the nature debate can enrich the discourse about (new) nature projects.

The above makes it clear that a place is not determined simply by space. Bastmeijer (2011) concludes that a place should be regarded 'as a place where an individual or a group of people can have a certain experience; it is the experience that makes the place into the place in question' (86). It is interesting that the respondents indicated that they had meaningful experiences in nature (cf. Figure 4 in chapter 6), however, there is a discrepancy: experiences suggested in the communication materials do not always match the experiences on the spot (chapter 5). More precisely, a number of key figures argue that public communication should suggest in advance how visitors will feel once they are on Tiengemeten; public communication should evoke the feeling of 'that's where I need to be; that's what I want to experience'. However, the results of this $\mathrm{PhD}$ thesis show that the words and images that are used in the public communication about the island do not always correspond with visitors' experiences once they are on Tiengemeten (chapter 5). In other words, how a place is experienced has an effect on how the communication about the place concerned is evaluated. This requires that the people involved in (communication) policy and strategy should consistently align their communication about the current state of development of nature projects. For this reason, the results of this $\mathrm{PhD}$ thesis suggest that (government) communication practitioners avoid pious words and promises and, rather, tell visitors what the nature reserve actually offers and minimise the use of so-called 'heavily value-laden language' (chapter 5). This is important for reasons of trust and is also necessary for avoiding scepticism by visitors and the risk of public communication becoming counterproductive.

\subsection{NATURE EXPERIENCES AND SECULAR SPIRITUALITY}

In this fourth and last theme, I would like to explore the concept of secular spirituality in relationship to nature experiences. Zwart (2011) states that land reclamation in the Netherlands can be linked to the Christianisation of de Lage Landen, mostly translated as the Low Countries. Therefore, it seems logical to expect the opposite: that land reclamation and the conversion of agricultural areas to nature such as, for example, Tiengemeten, go hand in hand with secularisation (chapters 4 and 6). This is also in keeping with Taylor (2012) who argues that wilderness areas, in this case (new), nature development projects, must be understood 'as temples

for those who have left behind conventional religions' (310). In an interview with PuurNature, the Natuurmonumenten members' magazine (winter 2015), Matthijs Schouten uses similar expressions. According to him, nature is important as an area of meaning for many people who have relinquished their belief. ${ }^{141}$ Taylor (2001b), therefore, signals an increasing interest in 'nature-based spiritualties' with the shared creed that 'the earth and all its life forms and processes are sacred' (238). Sacred, in this context, does not have a transcendent or theological interpretation by definition. It is about feelings of solidarity, recognition of the valuable character of and respect for this nature (cf. Taylor, 2004). This comes under the heading 'secular spirituality', a 'form of religion under conditions of secularity' (Mitchell, 2016: 3). Mitchell (2016) utilizes the term spirituality 'to indicate a distinctive and individualistic form of religiosity that is socially constructed and that operates in large part through avoidance of definition' (6). According to him, spirituality can best be regarded as 'an orientation to religiosity that is characterized by individuality, fluidity, and incommunicability'(6). He also argues that secularisation does not, by definition, mean a reduction in religiousness

141 On the one hand, he notes that we are becoming more secular - in the sense that traditional messages are embraced less - but,

at the same time, he signals a desire in people for a connection with something that is greater than themselves. According to him, people used to experience that in church, but nature currently takes that place. According to him, we risk being lost in a labyrinth of stories. Because we cannot live without a large, i.e., overarching story, nature has increasingly become this large story for people. A story that according to him, has to do with originality, with a world that is still in proper order, where there is harmony, which is a nice place to be. 142 Whereas people in the Neth42 Whereas people in the Nethestant or Catholic around 1900, there is currently, according to Schmeets \& Van Mensvoort (2015), deinstitutionalisation: 'people no longer comply with the traditional instructions of the church community' (2). Today's liberal society with its emphasis on individual freedom (of choice) is partly the cause of this. but rather means a change in the religious landscape. For the American context, he states:

Scholars have shown how 'secular' does not mean the absence of religion, but rather the reshaping f it. Indeed, more and more Americans describe themselves as 'spiritual' but not 'religious' declining to affiliate themselves with any particular religious roup (Mitchell, 2016:3).

The above also applies to the European situation. Research by Schmeets \& Van Mensvoort (2015) shows, for example, that the formal religious commitment in the Netherlands is increasingly waning: 'only a slight majority describe themselves as religious, and fewer han one in five people regularly attend a religiou service' (2). Yet, researchers conclude that religion in today's society still plays an important role, although today's religious landscape no longer corresponds to that of the past. ${ }^{142}$ Mitchell (2016) shows that, in this changing religious landscape, one thing should be borne in mind: the mediating role of the design and maintenance of nature reserves. 
Mitchell (2016) concludes in relationship to his research 'by focusing on spirituality in the context of the state management of space, one can better see how the secular works in practice to shape religious life' (3). In his book, he explores how the modern liberal state, as he refers to it, 'nurtures spirituality through the management of land, bodies, and sense perception' (3). It is this sentence that is important as part of this $\mathrm{PhD}$ thesis. The creation of 'new' nature areas such as, for example, Tiengemeten, is not a technically neutral activity. It is not 'only' nature; it is not just a 'public space'. It is a choice to, in the case of Tiengemeten, make farmers leave the island and create opportunities for nature (and associated recreation, nature experiences, etc.). This choice is based on dominant attitudes about nature that are the outcome of social developments and current attitudes toward human and nature relationships, and designing and maintaining nature areas in a certain way creates conditions for certain meaningful experiences. In other words: meaningful experiences in nature are, in a certain sense, mediated by design and maintenance. Maybe religiousness is no longer intrinsically linked to a specific community, as Taylor (2003) concludes, but where nature experiences are concerned, the individual nature of the experience is relative: there is always a government, a (nature) organisation, etc. that creates or has created 'conditions' (via policy, design, and management) for this, or as Mitchell (2016) states:

The national parks are managed environments. Each vista point, curve of the road, and recreational opportunity is an extension of a structure of authority, and each is designed to reinforce that structure through the management of experience. When visitors stand in awe before a grand panorama, enjoy the cool, quiet embrace of a redwood grove, or find time for self-reflection while walking on a smooth, well-marked trail, they do not simply connect to nature as something 'out there' nor simply to themselves as something 'inside'. Rather they invest in a certain way of experiencing, one that is maintained by the state that carves out these panoramas, keeps the forest quiet, and evens out the trails (Mitchell, 2016:193+194).

The above quote shows that we, with our designing vision and communication, can reap 'benefits' from nature. Stated differently, nature development projects such as, for example, Tiengemeten, can also play a mediating role. Paradoxically enough, this means that, in order to access the qualities of the nature that is referred to, we must, in a certain sense, 'own' it. We will need to create the conditions to enable accessing new or other layers of meaning. However, the question is whether it is the government's task to actively manage this. Restraint in this respect is desirable. It is not the government's task to encourage the transfer of so-called meaningful experiences. Its task is to act fairly regarding the various frames that play a role in the nature debate (chapter 2) and to attribute meaning to its actions within the framework of the nature policy (chapter 3).

\subsection{FINAL CONCLUSION}

This $\mathrm{PhD}$ thesis attempts to provide an answer to the question of whether and, if so, which (implicit) religious elements play a role in the communication about nature (policy) in the Netherlands. To conclude, this $\mathrm{PhD}$ thesis shows that religious elements play a role in the communication about nature. These are linked to meaningful experiences that people can have in nature. A religious depth dimension can be discovered in meaningful experiences. This religious depth dimension is the reason that there are 'religious subtexts' in the communication about nature. However, the word 'subtext' is crucial. The communication about nature is, to a certain extent, 'religionised' without any mention of a personal God or other supernatural agents. This $\mathrm{PhD}$ thesis also shows that the religious depth dimension does not explicitly come to the fore in what visitors are saying. This means that this $\mathrm{PhD}$ thesis, in addition to questioning the appropriateness of 'religious subtexts' in the communication about nature, also doubts whether those 'subtexts' are convincing from visitors' perspective. 


\section{References}

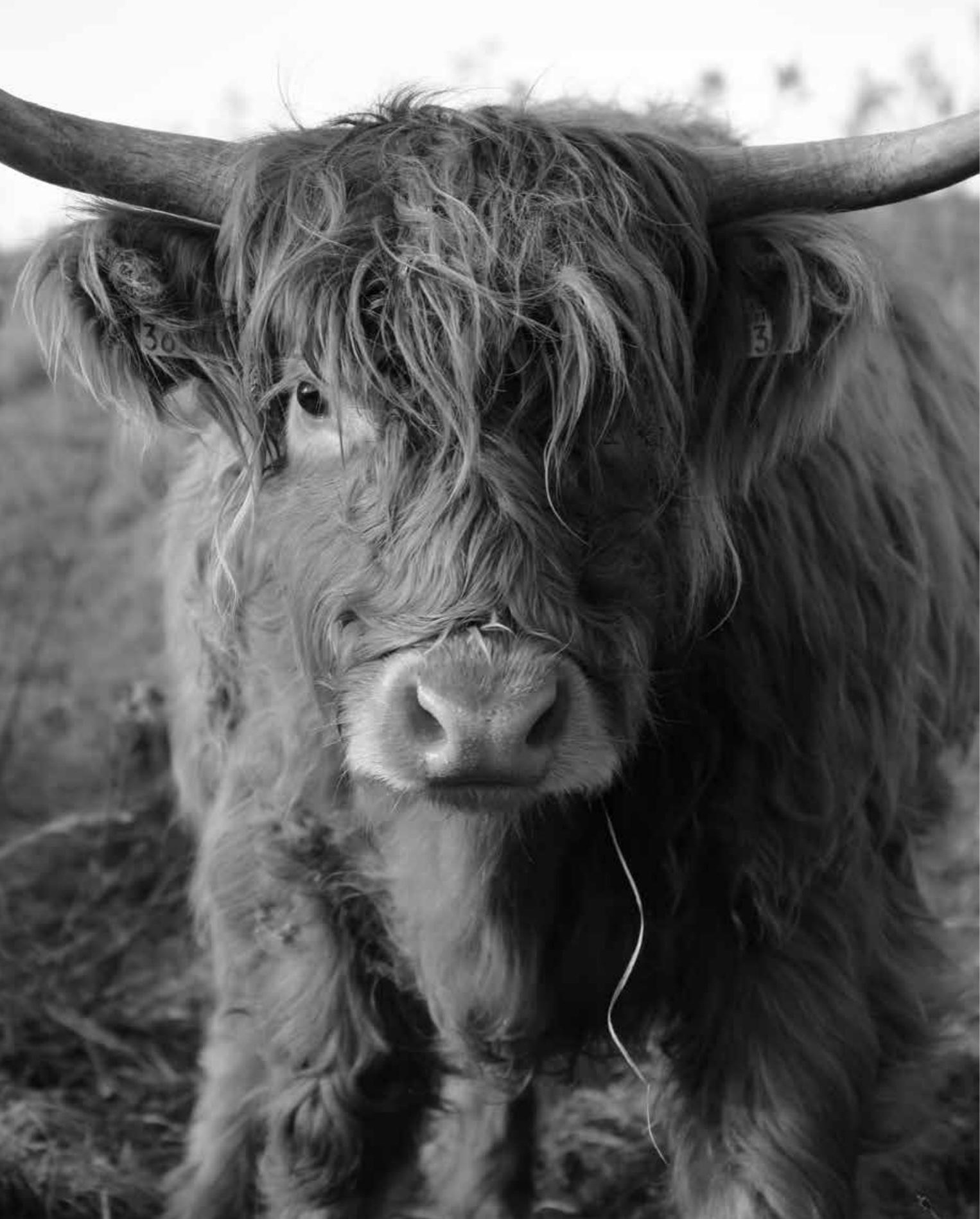

Aarts, N. (2012). Trending topics. In B. Ruler. (Ed.). Communicatie Nu; grootboek van het communicatievak (pp. 18-20). Amsterdam: Adformatie.

Aarts, N., Ruyssenaars, B., Steuten, Ch. \& Herzele, A. van (2015). Natuur en Beleid betwist; analyse van de aard en het verloop van online discussies over implementatie van natuurbeleid in Nederland. Den Haag: WRR.

Appolloni, S. \& Hrynkow, C. (2016). In Search of an Authentic Pax Gaia; Connecting Wonder, the Moral Imagination and Socio-Ecological Flourishing. Worldviews, 20 (2), 103-124.

Baarda, D. B., Goede, M. P. M. de, \& Teunissen, J. (2009). Basisboek kwalitatief onderzoek; handleiding voor het opzetten en uitvoeren van kwalitatief onderzoek. Groningen: Noordhoff.

Baarda, B., Bakker, E., Hulst, M. van der, Julsing, M., Fischer, T., Vianen, R. van \& Goede, M. de (2012). Basisboek methoden en technieken: kwantitatief praktijkgericht onderzoek op wetenschappelijke basis. Groningen: Noordhoff.

Balaban, D.C. (2008). The Framing of the Interpretation Frames Theory. Journal of Media Research, 1 (2), 9-13.

Bardoel, J. (2000). Culturr als confrontatie; publieke journalistiek in een private wereld. Den Haag: Ministerie van Onderwijs, Cultuur en Wetenschappen.

Basden, A. (2008). Philosophical Frameworks for Understanding Information Systems. New York: IGI Publishing.

Bastmeijer, K. (2011). Ieder een eigen plaats; over schaarste van ruimte en natuurlijke rijkdommen en de daaruit voortvloeiende 'territoriumdrift'. In R.J.G. van den Born, M. Drenthen, P. Lemmens \& T. van Slobbe. (Eds.). Plaats: Beschouwingen over verbondenheid met natuur en landschap (pp. 86-103). Zeist: KNNV Uitgeverij.

Berlin, I. ([1958] 2010). Twee opvattingen van vrijheid. Amsterdam: Boom.

Bekkers, V. (2001). Virtuele beleidsgemeenschappen. Over responsieve democratie en digitale participatie. Besturrskunde, 10 (6), 252-262.

Blokhuis, P. (2005). Homo communicans; de communicerende mens. In G.J. Buijs. (Ed.). Homo respondens; verkenningen rond mens zijn (pp. 181-190). Amsterdam: Buijten \& Schipperheijn.

Boer F. de \& Evers, J. (2007). Kwalitatief onderzoek, een korte inleiding. In J. Evers. (Ed.) Kwalitatief interviewen: kunst en kunde (pp. 3-22). Den Haag: Boom Lemma. 
Boer, T. A de \& Vries, J.R. de (2009). Genieten van nienwe natuur: recreatieonderzoek Oeverlanden Hollands Diep en Tiengemeten. Den Haag: DeltaNatuur/Wageningen University.

Boeije, H. (2005). Analyseren in kwalitatief onderzoek: denken en doen. Den Haag: Boom.

Bommel, S. van (2008). Understanding experts and expertise in different governance contexts. Wageningen: Wageningen University.

Born, R.J.G. van den (2008). Rethinking nature: Public visions in the Netherlands. Environmental Values, 17 (1), 83-109.

Born, R. J.G. van den \& Groot, M. de (2011). Favoriete plekken en binnendoorpaadjes. Op zoek naar de betekenis van landschap en verbondenheid met plaats. In R.J.G. van den Born, M. Drenthen, P. Lemmens \& T. van Slobbe. (Eds.). Plaats: Beschouwingen over verbondenheid met natuur en landschap (pp. 10-26). Zeist: KNNV Uitgeverij.

Boomars, L. (2001). De toeristische representatie van de Veluwe. In J. Lengkeek \& L. Boomars. (Eds.). Producten van verbeelding (pp. 51-61). Wageningen: Wageningen Universiteit.

Boutellier, H. (2011). De improvisatiemaatschappij; over de sociale ordening van een onbegrensde wereld. Den Haag: Boom Lemma.

Broer, N.A., J. Hoogland \& Stoep, J. van der (2017). Christian Higher Vocational Training in a Changing Context: Shifting from Pillars to Practices. Christian Higher Education, 16 (1-2), 53-64.

Buijs, A.E. (2009). Public Nature; social representations of nature and local practices. Wageningen: Wageningen Universiteit.

Buijs, A.E., Elands, B.H.M. \& Langers, F. (2009). No wilderness for immigrants: Cultural differences in images of nature and landscape preferences. Landscape and Urban Planning, 91 (3), $113-123$

Burkart, R. (2009). On Habermas; understanding and public relations. In Ø. Ihlen, B. van Ruler \& M. Fredriksson. (Eds.). Public relations and social theory; key figures and concepts (pp. 141-165). New York: Routledge.

Carey, J. W. (1992). Communication as culture; essays on media and society. New York: Routledge.

Casanova, J. (2006). Rethinking secularization: A global comparative perspective. Hedgehog Review, 8 (1-2),7-22.

Castells, M. (2009). Communication power. New York: Oxford University Press.

Craig, R.T. (1999). Communication Theory as a Field. Communication Theory, 9 (2),199-161.

Champ, J.G. (2009). Mediated Spectacular Nature: 'God-centered' and 'Nature-centered' Consumption of a Genre. Journal of Media and Religion, 8 (4), 226-241.

Chaplin, J. (2007). Public justice as a critical political norm. Philosophia Reformata, 7 (2), 130150.
Clouser, R. (1995). The myth of religious neutrality: An Essay on the Hidden Role of Religious Belief in Theories. Notre Dame: University of Notre Dame Press.

Cliteur, P. (1983). Een inleiding tot de filosofie en rechtstheorie van Herman Dooyeweerd. Radix, 9 (4),198-213.

Cooren, F. (2014). Organizational discourse. Cambridge: Polity Press.

Cronon, W. (1995). The Trouble with Wilderness, or Getting Back to the Wrong Nature. In W. Cronon. (Ed.). Uncommon Ground: Rethinking the Human Place in Nature (pp. 69-90). New York: W. W. Norton \& Co.

Darnton, A. \& Kirk, M. (2011). Finding Frames: New ways to UK public in global poverty. London: Bond

Deacon, M., Pickering, M., Golding, P., \& Murdock, G. (2010). Researching communications: A practical guide to methods in media and cultural analysis. New York: Bloomsbury.

Dingler, J. (2005). The discursive nature of nature: Towards a post-modern concept of nature. Journal of Environmental Policy \& Planning, 7 (3), 209-225.

Dooyeweerd, H. (1969). A New Critique of Theoretical Thought, vol. 2. The General Theory of the Modal Spheres. Phillipsburg: Presbyterian Publishing Company.

Drenthen, M. (2002). De Wilde Natuur en Verlangen naar Andersheid. In B. Voorsluis.

(Ed.). Zwijgende natuur: Natuurervaring tussen betovering en onttovering (pp. 65-86). Zoetermeer: Meinema.

Drenthen, M. (2003). Grenzen aan Wildheid: Wildernisverlangen en de betekenis van Nietzsches moraalkritiek voor de actuele milieu-ethiek. Budel: Damon.

Drenthen, M. (2011). Het leesbare landschap als woonplaats: Van lezend observeren naar plaatsverbondenheid. In R.J.G. van den Born, M. Drenthen, P. Lemmens \& T. van Slobbe (Eds.). Plaats: Beschouwingen over verbondenheid met natuur en landschap (pp. 121-141). Zeist: KNNV Uitgeverij.

Droogers, A. (2010). Zingeving als spel: Over religie, macht en speelse spiritualiteit. Almere: Parthenon.

Du Plooy, G. M. (2001). Communication research: Techniques, methods and applications. Lansdowne: Juta.

Eberg, J. (2006). De praktische wijhheid van overheidscommunicatie. Tijdschrift Bestuurskunde. $15(2), 64-74$.

Engelen, E., Keulartz, J. \& Leistra, G. (2008).European nature conservation policy-making; from substantive to procedural sources of legitimacy. In J. Keulartz \& G. Leistra. (Eds.).

Legitimacy in European nature conservation policy; case studies in multilevel governance (pp. 3-21). Heidelberg: Springer.

Entman, R.M. (1993).Framing: toward clarification of a fractured paradigm. Journal of Communication, 43 (4), 51-58. 
Ester, H. (2012). Romantiek versus Verlichting: een actuele uitdaging. Radix, 38 (1),4-14.

Feindt, P. H., \& Oels, A. (2005). Does discourse matter? Discourse analysis in environmental policy making. Journal of Environmental Policy \& Planning, 7 (3), 161-173.

Ferguson, T. \& Tamburello, J.A. (2015). The natural environment as a spiritual resource: A theory of regional variation in religious adherence. Sociology of Religion, 76 (3), 1-20.

Fischer, F. (2007). Deliberative Policy Analysis as Practical Reason: Integrating Empirical and Normative Arguments. In F. Fischer, G.J. Miller \& M.S. Sidney. (Eds.). Handbook of Public Policy Analysis (pp. 223-236). Boca Raton: CRC Press.

Freeman, R. E. (2010). Strategic Management: A Stakeholder Approach. Cambridge: Cambridge University Press.

Frith, K. (1998). Undressing the Ad: Reading Culture in Advertising. New York: Peter Lang.

Fusch, P. I., \& Ness, L.R. (2015). Are we there yet? Data saturation in qualitative research. The Qualitative Report, 20 (9), 1408- 1416.

Gadamer, H.G. ([1975] 2004). Truth and Method. London: Continuum.

Gibbs, G. (2007). Analysing Qualitative Data. London: Sage.

Glas, G. (2012). Competence development as normative practice - Educational reform in medicine as heuristic model to relate worldview and education. Koers - Bulletin for Christian Scholarship, 77(1), Art. \#411, 6 pages.

Glenny, L. (2010). Communication, Ethics and the Public Servant: Equality, Reciprocity, Truth and Authenticity. Adelaide: University of South Australia.

Goffman, E. (1959). Presentation of Self in Everyday Life. New York: Doubleday Anchor Books.

Gorter, H. P. (1986). Ruimte voor natuur, 80 jaar bezig voor de natuur van de toekomst.

"s-Graveland: Vereniging tot Behoud van Natuurmonumenten in Nederland.

Gorp, B. van (2004). Framing en het interpreteren van nieuws; een experimenteel onderzoek naar de effecten van frames. PSW paper 2004/6. Antwerpen: Universiteit Antwerpen.

Gorp, B. van (2007).The Constructionist Approach to Framing: Bringing Culture Back in. Journal of Communication, 57 (1), 60-78.

Groot, W.T. de \& Born, R.J.G. van den (2003).Visions of nature and landscape type preferences: an exploration in The Netherlands. Landscape and Urban Planning, 63 (3),127-138.

Groot, W.T., de (2011). Leren spreken over plaats. In gesprek met Jim Cheney over natuur, luisteren en doorvertellen. In R.J.G. van den Born, M. Drenthen, P. Lemmens \& T. van Slobbe. (Eds.). Plaats; beschouwingen over verbondenheid met naturr en landschap (pp. 105-118). Zeist: KNNV Uitgeverij.
Habermas, J. (1981). Theorie des kommunikativen handelns; zur kritik der funktionalistischen vernunft. Frankfurt: Suhrkamp.

Habermas, J. (1989). The structural transformation of the public sphere; An enquiry into a category of bourgeois society. Cambridge: MIT Press.

Hajer, M.A. (1995). The Politics of Environmental Discourse: Ecological Modernization and the Policy Process. Oxford: Oxford University Press.

Hajer, M.A. (2003). A frame in the fields: policymaking and the reinvention of politics. In M.A. Hajer \& H. Wagenaar. (Eds.). Deliberative Policy Analysis: Understanding Governance in the Network Society (pp. 88-110). Cambrigde: Cambridge University Press.

Hajer, M. A., \& Versteeg, W. (2005). A decade of discourse analysis of environmental politics: Achievement, challenges, perspectives. Journal of Environmental Policy \& Planning, 7 (3), 175184.

Hallahan, K. (1999). Seven Models of Framing: implications for Public Relations. Journal of Public Relations Research, 11 (3), 205-242.

Hartig, T., Mitchell, R., de Vries, S., \& Frumkin, H. (2014). Nature and Health. Annual Review of Public Health, 35 (1), 207-228

Hobbs, R. J., Arico, S., Aronson, J., Baron, J. S., Bridgewater, P., Cramer, V. A, ... Zobel, M. (2006). Novel ecosystems: Theoretical and management aspects of the new ecological world order. Global Ecology and Biogeography, 15 (1), 1-7.

Hobbs, R. J., Higgs, E., \& Harris, J.A. (2009). Novel ecosystems: Implications for conservation and restoration. Trends in Ecology and Evolution, 24 (11), 599-605.

Hobbs, R. J., Cole, D. N., Yung, L., Zavaleta, E. S., Aples, G. H., Chapin, F. S., ... Woodley, S. (2010). Guiding concepts for park and wilderness stewardship in an era of global environmental change. Frontiers in Ecology and the Environment, 8 (9), 483-490.

Hobbs, R. J., Higgs, E., Hall, C. M., Bridgewater, P., Chapin, F. S., Ellis, E. C., ...Yung, L. (2014). Managing the whole landscape: Historical, hybrid, and novel ecosystems. Frontiers in Ecology and the Environment, 12 (10), 557-564.

Holling, C. S. (2001). Understanding the complexity of economic, ecological and social systems. Ecosystems, 4 (5), 390-405.

Hoogland, J. \& Jochemsen, H. (2000). Professional Autonomy and the Normative Structure of Medical Practice. Theoretical Medicine and Bioethics, 21 (5), 457-475.

Hoover, S. M. (1997). Religion in a Media Age. Public lecture on Media, Religion \& Culture at the University of Edinburgh, 4 March

Hoover, S. M. (2002).The Culturalist Turn in Scholarship on Media and Religion. Journal of Media and Religion, 1 (1), 25-36.

Hoover, S. M. (2006). Religion in Media Age. New York: Routledge. 
Horn, G, van \& Hausdoerffer, J. (2017). Wildness: Relations of People and Place, Chicago: University of Chicago Press.

Hycner, R.H. (1999). Some guidelines for the phenomenological analysis of interview data', in A. Bryman \& R.G. Burgess. (Eds.) Qualitative Research (pp. 143-164). London: Sage.

Ihlen, Ø. \& Ruler, B. van (2007). How public relations works: Theoretical roots and public relations perspectives. Public Relations Review, 33 (3), 243-248.

Jacobs, M. (2002). Landschap.Wageningen: Alterra.

Jackson, S. T., \& Hobbs, R.J. (2009). Ecological restoration in the light of ecological history. Science, 325 (5940), 567-569.

Jacobson, Th. \& Kolluri, S. (1999). Participatory communication as communicative action. In T. Jacobson \& J. Servaes. (Eds.). Theoretical approaches to participatory communication (pp. 265280). Cresskill: Hampton Press.

James, W. ([1901] 1999). The varieties of religious experience. New York: The Modern Library.

Jeffrey, L. M. \& Brunton, M.A. (2011). Developing a framework for communication management competencies. Journal of Vocational Education and Training, 63 (1), 57-75.

Jochemsen, H. (2006). Normative Practices as an Intermediate between Theoretical Ethics and Morality. Philosophia Reformata, 71 (1), 96-112.

Jochemsen, H., Kuiper, R. \& Muynck, B. de (2006). Een theorie over praktijken. Amsterdam: Buijten \& Schipperheijn.

Jong, D. de (1999). Tussen natuurontwikkeling en Landschaftsschutz: sociaal-cognitieve configuraties in het grensoverschrijdende natuurbeleid. Delft: Eburon.

Jumelet, L. \& Wassenaar, I. (2003). Overheidscommunicatie. Utrecht: Thieme Meulenhoff.

Keil, R., \& Debanné, A. (2005). Scaling discourse analysis: Experiences from Hermanus, South Africa and Walvis Bay, Namibia. Journal of Environmental Policy \& Planning, 7 (3), 257-276.

Kempton, W.M., Boster, J.S. \& Hartley, J.A. (1995). Environmental values in American culture. Cambridge: MIT Press.

Keulartz, J. (2000). Naar een 'beschaafde' strijd om de natuur. In J. Keulartz. (Ed.). Rustig, ruig en rationeel: Filosofische debatten over de verhouding cultuur-natuur (pp. 75-96). Baarn: Kasteel Groeneveld/Staatsbosbeheer/Wageningen Universiteit.

Keulartz, J., Windt, H. van der \& Swart, S. (2002). Natuurbeelden en natuurbeleid. Filosofie \& Praktijk, 23 (1), 3-21

Keulartz, J., Windt, H. van der \& Swart, S. (2004). Concepts of nature as communicative devices: The case of Dutch nature policy. Enviromental Values, 13 (1), 81-99.

Keulartz, J. (2005). Werken aan de grens; de spanning tussen diversiteit en duurzaamheid. Nijmegen: Radboud Universiteit.
Keulartz, J. (2009a).Van ecologisch herstel tot ecologisch ontwerp; natuur in het tijdperk van globale veranderingen. Filosofie \& Praktijk, 30 (6), 44-59.

Keulartz, J. (2009b). European Nature Conservation and Restoration Policy: Problems and Perspectives. Restoration Ecology, 17 (4), 446-450.

Keulartz, J. (2011). Plaats tussen Utopia en Nostalgia. In R.J.G. van den Born, M. Drenthen, P. Lemmens \& T. van Slobbe. (Eds.). Plaats: Beschouwingen over verbondenheid met natuur en landschap (pp.164-178). Zeist: KNNV.

Klop, C.J. (1996). Politieke filosofie. In R. van Woudenberg (Ed.). Kennis en werkelijkheid; tweede inleiding tot een Christelijke filosofie (pp. 310-341). Amsterdam: Buijten \& Schipperheijn.

Kockelkoren, P. (2000). Een leesbaar bokrijk. In J. Keulartz. (Ed.). Rustig, ruig en rationeel: Filosofische debatten over de verhouding cultuur-natuur (pp. 19-41). Baarn: Kasteel Groeneveld/ Staatsbosbeheer/Wageningen Universiteit.

Komter, A. (2003). Solidariteit en de gift; sociale banden en sociale uitsluiting. Amsterdam: Amsterdam University Press.

Kooij, P. (1999). Mythen van de groene ruimte. Inaugural lecture on Agricultural History at Wageningen University, 10 June.

Koppen, C S.A. van (2002). Echte natuur: Een sociaal theoretisch onderzoek naar natuurwaardering en natuurbescherming in de moderne samenleving. Wageningen: Wageningen University.

Krabbe, H. (2011). Communicatieprofessionals en ethiek, morele communicatie-vraagstukken in organisaties. Amsterdam: Boom Lemma.

Kuindersma, W, Kistenkas, F.H. \& Apeldoorn, R.C. van (2004). De transformatie van Nederlands natuurbeleid door Europees recht. Wageningen: Natuurplanbureau/Wageningen Universiteit.

Lakoff, G. (2006).Thinking Points; Communicating our American values and vision. New York Farrar, Straus and Giroux.

Latour, B. (1993). We have never been modern. Cambrigde: Harvard University Press.

Lemaire, T. (2002). Sublieme natuur als grenservaring van moderniteit. In M. Coolen \& K. va der Wal. (Eds.). Het eigen gewicht van de dingen. Milieufilosofische opstellen (pp. 13-42). Damon: Bundel.

Lemaire, T. 2007 [1970]. Filosofie van het landschap. Amsterdam: Ambo.

Lemaire, T. (2011). Bezielde plekken en onplaatsen. In R.J.G. van den Born, M. Drenthen, P. Lemmens \& T. van Slobbe. (Eds.). Plaats: Beschouwingen over verbondenheid met natuur en landschap (pp. 27-28). Zeist: KNNV.

Linell, P. (1998). Approaching dialogue: Talk, interaction and contexts in dialogical perspectives. Amsterdam: John Benjamins.

Lowenthal, D. (2007). Living with and Looking at Landschap. Landscape Research, 32 (5), 635- 
MacIntyre, A. (1984). AfterVirtue, A study in moral theorie. London: Duckworth.

Mason, M. (2010). Sample size and saturation in PhD studies using qualitative interviews. Forum Qualitative Sozialforschung/ Forum Qualitative Social Research, 11 (3), Art. 8.

Marshall, C. \& Rossman, G.B. (1999). Designing Qualitative Research. Thousand Oaks: Sage. McGrath, A.E. (2001). A Scientific Theology, Vol. 1, Nature. Edinburgh: T. \& T. Clark. Metz, T. (1998). Nieuwe Natuur, reportages over veranderend landschap. Amsterdam: Ambo.

Meyer, B. (2012). Mediation and the Genesis of Presence: Towards a Material Approach to Religion. Utrecht: Universiteit Utrecht.

Middel, R. (2002). Daar hebben we toch communicatie voor; een beschouwing over de toegevoegde waarde van een discipline. Utrecht: Hogeschool Utrecht.

Ministerie van Algemene Zaken (1970). Openheid, openbaarheid; rapport van de commissie heroriëntatie overheidsvoorlichting. Den Haag: Ministerie van Algemene Zaken.

Ministerie van Algemene Zaken (2001). In dienst van de democratie; rapport van de Commissie Toekomst Overheidscommunicatie. Den Haag: Ministerie van Algemene Zaken.

Ministerie van Economische Zaken (2014). Natuurlijk Verder; Rijksnatuurvisie 2014. Den Haag: Ministerie van Economische Zaken.

Ministerie van LNV (1989). Natuurontwikkeling: een verkennende studie. Den Haag: Ministerie van LNV.

Ministerie van LNV (1990). Natuurbeleidsplan: regeringsbeslissing. Den Haag: SDU.

Ministerie van LNV (2000). Natuur voor mensen, mensen voor natuur: nota natuur, bos en landschap in de 21e eeuw. Den Haag: Ministerie van LNV

Ministerie van LNV (2005). Naar een nieuwe natuur; over natuurontwikkeling in Nederland. Den Haag: Ministerie van LNV.

Mitchel, K. (2016). Spirituality \& the State; Managing Nature and Experiences in America's National Parks. New York: New York University Press.

Mouw, R. \& Griffioen, S. (1993). Pluralisms and Horzons: An Essay in Christian Public Philosophy. Grand Rapids: W.B. Eerdmans Publishing Company.

Nash, R.F ([1969] 2001). Wilderness and the American Mind. New Haven: Yale University Press.

Natuurmonumenten (2011). Natuurbehoud Magazine, vol. 2. ‘s-Gravenland: Vereniging to Behoud van Natuurmonumenten in Nederland.

Natuurmonumenten (2012). Kom naar Tiengemeten. 's-Gravenland: Vereniging tot Behoud van Natuurmonumenten in Nederland.
Natuurmonumenten (2014). Natuurbeleving nader bekeken. 's-Graveland: Vereniging tot Behoud van Natuurmonumenten in Nederland.

Natuurmonumenten (n.d.). Weelde, Wildernis en Weemoed; ontdek natuureiland Tiengemeten. s- Gravenland: Vereniging tot Behoud van Natuurmonumenten in Nederland.

Nationaal Groenfonds (2010). Publiek geheim; succes van de EHS. Amsterdam: Nationaal Groenfonds.

Naugle, D.K. (2002). Worldview; the History of a Concept. Grand Rapids: W.B. Eerdman Publishing Company.

Noordhoff, I. (2011). Natuurmakers; heroverd landschap van Rottum tot Grensmaas. Amsterdam/ Antwerpen: Uitgeverij Atlas.

Note, N., Fornet-Betancourt, R., Estermann, J., \& Aerts, D. (2009). Worldview and cultures: philosophical reflections from an intercultural perspective. New York: Springer.

Olsthoorn, A.C.J.M. \& Velden, J.H. van der (2007). Elementaire communicatie; strategie, beleid, uitvoering. Utrecht: Thieme Meulenhoff.

Otto, R. ([1917] 1923). The idea of the holy: An inquiry into the non-rational factor in the idea of the divine and its relation to the rational. London: Oxford University Press.

Oudemans, Th. C.W. (1988). Gadamers wijsgerige interpretatiekader. In Th. De Boer. (Ed.). Hermeneutiek; filosofische grondslagen van mens- en cultuurwetenschappen (pp. 54-89). Amsterdam: Boom.

Peterson, M.N. \& Liu, J. (2008). Impacts of Religion on Environmental Worldviews: The Teton Valley Case. Society and Natural: An International Journal, 21 (8), 704-718.

Pickering, M. \&Keightley, E. (2006).The modalities of nostalgia. Current Sociology, 54 (6), 919941

Plooij, F. (2011). Onderzoek doen, een praktische inleiding in onderzoeksvaardigheden. Amsterdam: Pearson Education.

Pol, B., Swankhuisen, C. \& Vendeloo, P. van (2007). Nieuwe aanpak in overheidscommunicatie; mythen, misverstanden en mogelijkheden. Baarn: Uitgeverij Coutinho.

Pol, B. (2008). Tussen professoren en professionals; van wetenschappelijk onderzoek naar beïnvloedende overheidscommunicatie. Utrecht: Hogeschool Utrecht.

Posthoorn, R. (2000). Ontwikkelvisie Tiengemeten. Eiland van Wildernis, Weelde en Weemoed. Den Haag: Ministerie van Verkeer en Waterstaat.

Reckwitz, A. (2002). Toward a theory of social practices: a development in culturalist theorizing. European Journal of Social Theory, 5 (2), 243-263.

Ricoeur, P. (1991). From text to action: essays in hermeneutics II. Northwestern University Press: Evanston. 
Robson, C. (2002). Real World Research. Oxford: Blackwell.

Roothaan, A. (2005). Terugkeer van de natuur; de betekenis van natuurervaring voor een nieuwe ethiek. Kampen: Klement.

Ross-Bryant, L. (2013). Pilgrimage to the National Parks: Religion and Nature in the United States. New York: Routledge.

Ruler, B. van \& Verčič, D. (2005). Reflective communication management: Future ways for public relations research. In P. J. Kalbfleisch. (Ed.). Communication yearbook (pp. 239-274). Mahwah: Erlbaum.

Ruler, B. van (2007). Communicatiemanagement; speelvelden en beleid. In R. van Es. (Ed.). Communicatie en ethiek; organisaties en hun publieke verantwoordelijkheid (pp. 71-82). Amsterdam: Boom.

Ruler, B. van \& Verĉiĉ, D. (2012). Reflective communication management. Future ways for public relations research. In B. van Ruler. (Ed.). Met het oog op communicatie. Reflecties op het communicatievak (pp. 76-108). Den Haag: Boom Lemma.

Saane, J. van (2002). De natuur in religieuze verbeelding. In B. Voorsluis. (Ed.). Zwijgende natuur: Natuurervaring tussen betovering en onttovering (pp. 48-64). Zoetermeer: Meinema.

Saunders, M., Lewis,P., Thornhill, A., Booij, M. \& Verckens, J.P. (2011). Methoden en technieken van onderzoek. Amsterdam: Pearson Education.

Schama, S. (1998). Landschap en Herinnering. Amsterdam/Antwerpen: Uitgeverij Contact.

Schmeets H. \& Mensvoort, C. van (2015). Religieuze betrokkenheid van bevolkingsgroepen 2010-2014. Den Haag: Centraal Bureau voor de Statistiek.

Schouten, M.G.C. (2002). Tussen Arcadië en Wildernis. In B. Voorsluis. (Ed.). Zwijgende natuur: Natuurervaring tussen betovering en onttovering (pp. 16-37). Zoetermeer: Meinema.

Schouten, M.G.C. (2005). Spiegel van de natuur: het natuurbeeld in cultuurhistorisch perspectief. Utrecht: KNVV/Staatbosbeheer.

Servaes, J. (1999). Communication for development: one world, multiple culture. Creskill: Hampton

Sewell, W.H. (1992). A theory of structure: Duality, agency, and transformation. American Journal of Sociology, 98 (1),1-29.

Sinke, D. (dir.) (2010). Wistful Wilderness. Film trailer. Amsterdam: SNG Film.

Smaling, A. (2011). Constructivisme in soorten. In A. Smaling \& F. de Boer (Eds.), Benaderingen in kwalitatief onderzoek (pp.79-92). Den Haag: Boom.

Strauss, D. (2009). Philosophy: Discipline of the Disciplines. Grand Rapids: Paideia Press.
Stoep, J. van der (2006). Norms of Communication and the Rise of the Network Society. In S. Strijbos \& A. Basden. (Eds.). In Search of an Integrative Vision for Technology (pp. 133-146). New York: Springer Verlag.

Stoep, J. van der (2011). Social Media and the Normative Structure of Corporate Communication. Paper presented at the international conference The Future of Creation Order, Amsterdam: VU University, 18 August.

Stoep, J. van der (2016). Deugt het verhaal? Herontdekking van het ambacht in journalistiek en communicatie. Ede: Christelijke Hogeschool Ede.

Stolow, J. (2005). Religion and/as media. Theory, Culture \& Society, 22 (4), 199-145.

Svarstad, H., Petersen, L. K., Rothman, D., Siepel, H., \& Wätzold, F. (2008). Discursive biases of the environmental research frame-work DPSIR. Land Use Policy, 25 (1), 116-125.

Swanborn, P.G. (2008). Case-study's: wat, wanneer en hoe?. Den Haag: Boom Onderwijs.

Swart, J.A.A., Windt, H.J. van der \& Keulartz, J. (2001).Valuation of nature in conservation and restoration. Restoration Ecology, 9 (2), 230-238.

Tannen, D., Hamilton, H. E., \& Schiffrin, D. (2015). The handbook of discourse analysis. Chichester: Wiley Blackwell.

Tench, R. \& Yeomans. L. (2006). Exploring Public Relations. London: Prentice Hall.

Taylor, B. (2001a). Earth and nature-based spirituality (part I): From deep ecology to radical environmentalism. Religion, 31 (2), 175-193.

Taylor, B. (2001b). Earth and nature-based spirituality (part II): From Earth First! and bioregionalism to scientific Paganism and the New Age. Religion, 31 (3), 225-245.

Taylor, B. (2004). A green future for religion?. Futures, 36 (9), 991-1008.

Taylor, B. (2010). Dark Green Religion; Nature Spirituality and the Planetary Future. Berkeley: University of California Press.

Taylor, B. (2012). Wilderness, spirituality and biodiversity in North America: Tracing an environmental history from Occidental roots to Earth Day. In L. Felt. (Ed.).Wildernes mythologies: Wilderness in the history of religion (pp. 292-324). Berlin: De Gruyter.

Taylor, C. (1989). Sources of the Self: The Making of the Modern Identity. Cambridge: Cambridge University Press.

Taylor, C. (1991). The ethics of authenticity. London: Harvard University Press.

Taylor, C. (1994). The Politics of Recognition. In A. Gutman. (Ed.). Multiculturalisn: Examining the Politics of Recognition (pp. 25-75). Princeton: Princeton University Press.

Taylor, C. (2003). Wat betekent religie vandaag?. Kapellen: Pelckmans/ Klement.

Thayer, R. (2003). LifePlace. Berkeley: University of California Press. 
Vandermause, R.K. \& Fleming, S.E. (2011). Philosophical Hermeneutic Interviewing. International Journal of Qualitative Methods, 10 (4), 367-377.

Vera, F. (2000). Het primitieve natuurbeeld. In J. Keulartz. (Ed.), Rustig, ruig en rationeel: Filosofische debatten over de verhouding cultuur-natuur (pp. 43-61). Baarn: Kasteel Groeneveld/ Staatsbosbeheer/Wageningen Universiteit.

Verhoeven, N. (2007). Wat is onderzoek? Praktijkboek methoden en technieken voor het hoger onderwijs. Den Haag: Boom Onderwijs.

Verkerk, M. J. (2014). A philosophy-based 'toolbox' for designing technology: the conceptua power of Dooyeweerdian philosophy. Koers - Bulletin for Christian Scholarship, 79 (3), Art. \#2164, 7 pages.

Verkerk, M.J., Hoogland, J., Stoep, J van der \& Vries, M.J. de (2016). Philosophy of technology? an introduction for technology and business students. New York: Routledge.

Verschuren, P. \& Doorewaard, H. (2000). Het ontwerpen van een onderzoek. Utrecht: Lemma.

Voye, L. (2004). A Survey of Advances of in the Sociology of Religion (1980-2000). In P. Antes, A.W. Geertz, \& R.R. Warne. (Eds.). New Approaches to the Study of Religion (pp. 195228). Vol. 2. Textual, Comparative, Sociological and Cognitive Approaches. Berlin: W. de Gruyter.

Vogt, W. P. (1999). Dictionary of statistics and methodology: A nontechnical guide for the social sciences. London: Sage.

Vreke, J., Donders, J.L.M., Elands, B.H.M., Goossen, M., Langers, F., Niet, R. de \& Vries, S. de (2007). Natuur en landschap voor mensen. Wageningen: Alterra/MNP/Wageningen Universiteit.

Vries, J. de (2014). Understanding trust. Longitudinal studies on trust dynamics in governance interactions. Wageningen: Wageningen University.

VVV Zuid Holland Zuid (2012). Toeristische Gids Nationaal Landschap Hoeksche Waard. Dordrecht: VVD Zuid Holland Zuid.

Waaijman, C. J. (1994). Milieuspiritualiteit. Nijmegen: Titus Brandsma Instituut.

Waaijman, C. J. (2000). Spiritualiteit: Vormen, grondslagen, methoden. Kampen: Kok.

Willis, A. (2011). Re-Storying Wilderness and Adventure Therapies: Healing Places and Selves in an Era of Environmental Crises. Journals of Adventure Education \& Outdoor Learning, 11 (2), 91-108.

Windt, H. van der, Swart, S. \& Keulartz, J. (2007). Nature and landscape planning: Exploring the dynamics of valuation, the case of the Netherlands. Landscape and Urban Planning, 79 (3-4), $218-228$.

Winkelaar, P. (2005). Anders dan we denken: Een geseculariseerde benadering van het religieuze. Amsterdam: SWP Uitgeverij.
Woud, A. van der (2007). De blanke top der duinen; mooi Nederland en zijn historie. Den Haag: KVAN/Nationaal Archief.

Wolf, J. A. (2012). Wildernis in Nederland; tussen droom en werkelijkheid: Een onderzoek naar de fysieke wildernis en de beeldvorming over wildernis in Nederland in verleden en heden. Groningen: University of Groningen.

Woudenberg, R. van (1992). Gelovend denken: inleiding tot een christelijke filosofie. Amsterdam: Buijten \& Schipperheijn.

Yin, R.K. (2009). Case Study Research: design and methods. London: Sage.

Ziel, T.J. van der (2003). Verzet en Verlangen; de constructie van nieuwe ruraliteiten rond de $m k z-$ crises en de trek naar het platteland. Wageningen: Wageningen Universiteit.

Zouwen, M. van der (2006). Nature Policy between Trends and Traditions: dynamics in nature policy arrangements in The Yorkshire Dales, Donnana and the Veluwe. Delft: Eburon.

Zwanniken, T. (2001). Ruimte als voorraad? Ruimte voor variëteit!; de consequenties van discoursen 'ruimte als voorraad' voor het rijks ruimtelijk beleid. Nijmegen: Radboud University.

Zwart, J (1994). De weerbastige staat. Dooyeweerds zoektocht naar het juiste begrip van de staat. In H.G. Geertsema, J. Zwart, J. de Bruijn, J. van der Hoeven \& A. Soeteman (Eds.) Herman Dooyeweerd 1894-1977, breedte en actualiteit van zijn filosofie pp. 5-76). Kampen: Kok.

Zwart, H. (2002). Een morele geschiedenis van het Nederlandse landschap. In B. Voorsluis. (Ed.). Zwijgende natuur: Natuurervaring tussen betovering en onttovering (pp. 38-47). Zoetermeer: Meinema.

Zwart, H. (2011). De tuin als landschapsarchief, laboratorium en dialoog. In R.J.G. van den Born, M. Drenthen, P. Lemmens \& T. van Slobbe. (Eds.). Plaats: Beschouwingen over verbondenheid met natuur en landschap (pp. 72-85). Zeist: KNNV.

Zweers, W. (1989). Grondhoudingen ten opzichte van natuur. Heidemij Tijdschrift, 6, 74-80. 
you may assume that social legitimacy follows. Communicating convincingly and truthful means that the communication of the government is consistent with its 'telos', consistent with how the government is acting at this time and consistent with how it has communicated before. It is important to distinguish the aspect of social legitimacy from the question of whether all stakeholders substantively agree. If the communication is sound, you may assume that the stakeholders are experiencing the input of the government as a legitimate contribution and accept potential decisions, even if they do not agree. Democracy is not so much about citizens agreeing with the actual policy of the government and government's actions, but that they can trust the preparatory democratic decision-making.

Chapter 4 describes the different ways in which nature is regarded in the Dutch nature policy. An important marking point are the 80s and 90s of the past century. Since then, a revival of the wilderness view of nature is visible in particular. Where in the past, the wilderness in nature was recognised, since the 80s and 90s, more and more nature is 'made' to our 'image of wilderness'. This is essentially a paradox: nature development connects to current ideas about technology, while it strives for a prehistoric type of nature that is independent of humanity. Therefore, in a sense, there is a technical approach to nature in the Netherlands.

Chapter 4 also concluded that meaningful experiences are found in nature. There is a variety of names for this to be found in the literature, such as: sublime experiences, experiencing the numinous, spiritual/religious experiences, epiphany, etc. In general, you could speak of transcendental experiences: experiences that take people beyond what they can rationally grasp and where they feel addressed by 'the Other', whatever that may be, but which is experienced or regarded as holy. These kinds of experiences ensure that elements with a religious connotation, so-called 'religious subtexts', can be found in communication about nature. This $\mathrm{PhD}$ thesis shows that these 'religious subtexts' are not necessarily theological in nature. Rather, the examples in chapter 4 make clear that in many cases, there is no mention of a personal God or any other supernatural reference.

Based on a qualitative case study about Tiengemeten, chapter 5 compares the experiences of visitors with the discourse of the policy and communications practitioners, the so-called key figures. Tiengemeten was chosen because this nature development project exemplifies the current thinking about nature in the Netherlands. A certain reluctance can be observed among the respondents in the use of the term 'true' nature. For respondents, 'true' nature often has the connotation of spontaneous and autonomous development, rather than pure or original.
Chapter 5 concludes that among the respondents, the wilderness view of nature is dominant in combination with what is called a light version of the Arcadian view of nature. There is no demonstrable difference between visitors and the key figures here. You might say that there is a hybridisation of wilderness and Arcadian among the respondents, of rough and quiet, of appreciation of new nature combined with melancholic tendencies toward the past.

Chapter 5 signals a discrepancy between what the key figures communicate on the one hand, and visitors of Tiengemeten experience on the other hand. Key figures indicate that the public communication should evoke the feeling of 'I want to be there, that is what I want to experience'. But the case study regarding Tiengemeten shows that words and images used in the discourse do not always correspond with the experiences of visitors once they get to the described location. The experiences the communications suggest not always match the experiences on site. In other words, experiencing a place influences how the communication about it are evaluated. Therefore, chapter 5 advocates continuous streamlining of the public communication and the current state of the nature development. It is advised to avoid 'big words' or 'heavily value-laden' communications and connect to what nature development projects actually produce. This is important both in terms of trust, to avoid scepticism among visitors, and in terms of not running the risk that the public communications become counterproductive.

Also based on a qualitative case study of Tiengemeten, chapter 6 describes to what extent depth layers are visible in the communications of visitors of Tiengemeten. What is striking is that words and images visitors use to describe their experiences mainly fit the wilderness view of nature. Looking at what experiences can be identified in experiences expressed in words, what stands out is that first, there are experiences that are strongly connected to the specific context of Tiengemeten. Feelings of loss of the (agricultural) identity and experiences that have to do with the fact that Tiengemeten is an island. The crossing by ferry is crucial to many respondents. It gives them feelings of 'letting go of one world and entering another'. One of the respondents also spoke of this in terms of a different dimension of existence. But there were also experiences that expressed what we call the appealing force of nature. This involves feelings of recharging yourself, finding yourself (again), etc. in nature, as well as experiences that relate to experiencing the grandeur and the overwhelming aspect of nature. The latter category is more fitting for the wilderness view of nature, while mainly the experience of loss of the (agricultural) identity fits an Arcadian view of nature. 
The most important results of this $\mathrm{PhD}$ thesis are discussed in chapter 7. This is done based on four themes. In the first theme, normative character of communication, it is argued that the role of communication practitioners is to signal, articulate, and interpret normative elements in the discourse. In other words, to make (non-)congruent frames explicit and clarifying the associated world views in the discourse, including that of the government itself. The government has to be impartial as possible in its communications, but the communications about nature shows that there are questions to be asked about this neutrality. Although not explicit, but through the communications of NGOs, who operate as delegated executors of the Dutch nature policy in the context of this $\mathrm{PhD}$ thesis, certain images, i.e. frames regarding nature are communicated. In other words, it is striking that in the communication of the NGOs, there are 'religious subtexts': there mostly seems to be an appeal to 'an escape from everyday life', nature is presented as the 'better world', and a sort of promise is made in the communications - in nature you can find yourself (again)

In the second theme, 'religious subtexts' in the communication, the question is raised to what extent the government, based on its alleged neutrality, should condition the communication of NGOs. Otherwise stated, do 'religious subtexts' belong to communication about the effects of the government policy? In the case of this $\mathrm{PhD}$ thesis NGOs are de facto executors of the Dutch nature policy. Here, tension can be observed. If nature conservation NGOs (explicitly) communicate a specific vision about nature, using 'religious subtexts', the government appears to support these 'subtexts'. For nature conservation NGOs, it is appropriate to put forth a certain opinion to raise support for their actions among the public. However, in the second theme it is argued that it is not the responsibility of the government to promote a specific religiously phrased view of nature and nature policy. In doing so, the government uses its power for inappropriate purposes. Hence, this second theme reveals a necessity for reflection on the relationship between government and NGOs regarding their communication, i.e., awareness of distinction and a need for mutual adjustment in the case of close cooperation.

In the third theme, paradox in nature experiences, the results of this $\mathrm{PhD}$ thesis are placed in a broader cultural context with respect to nature development. In this theme, a paradox is highlighted: creating nature 'according to our view of nature' and, simultaneously, wanting to experience wilderness-nature, preferably without too much human influence. This paradox appears to form a cultural basis for many new nature development projects. In other words, nature development is no longer just driven by ecological interests. In today's 'wilderness desire', a certain form of anthropocentric thinking also manifests, because it focuses on the human experience of nature. In addition, because (new) nature projects can be places to have meaningful experiences, in this theme, it is concluded that (new) nature projects, such as Tiengemeten, not only have ecological value, but societal value as well.

In the fourth theme, nature experiences and secular spirituality, it is argued that in a secular society, we should not lose sight of the mediating role of creating and maintaining nature parks. The construction of (new) natural areas is not a technically neutral activity. It is not 'just' nature, or just a 'public space'. Designing or maintaining natural areas in a certain way can create conditions for certain meaningful experiences. In other words: meaningful experiences in nature are mediated by design and maintenance. Perhaps religiosity is no longer intrinsically linked to a specific community, but in the context of nature experiences, the individual aspect of the experience is relative: there is always a government, a nature organisation, etc. which creates or has created 'conditions' (through policy, design, and maintenance). With our designing vision and communication, we can reap the 'benefits' of nature. With this conclusion, this $\mathrm{PhD}$ thesis shines a different light on the concept of nature development and, indirectly, on the Dutch nature policy.

Finally, based on five studies, appearing as separate chapters 2 to 6 , this $\mathrm{PhD}$ thesis has collected both theoretical and empirical evidence to answer the question of whether (implicit) religious elements play a role in the communication regarding nature in the Netherlands. The conclusion shows that religious elements play a role in the communication regarding nature. These appear to be associated with meaningful experiences that people can have in nature. A religious depth dimension can be discovered in meaningful experiences. This religious depth dimension is the reason why there are 'religious subtexts' in the communication regarding nature. It is important to emphasise the word 'subtext', because the communication regarding nature is 'religionised' to some extent, but there is no mentioning of a personal God or other reference to a supernatural reality. This $\mathrm{PhD}$ thesis also shows that the religious depth dimension does not explicitly come to the fore in what visitors are saying. The result is that this thesis, in addition to the question regarding the appropriateness of 'religious subtexts' in the communication regarding nature, also questions whether these 'subtexts' are convincing from the perspective of the visitors. 


\section{Samenvatting}

Dit proefschrift begint in hoofdstuk 1 met de constatering dat er met betrekking tot het Nederlandse natuurbeleid in de afgelopen dertig á veertig jaar een verandering heeft plaatsgevonden. Van een focus op behouden en beschermen van bestaande natuur naar het ontwikkelen van zogenoemde 'nieuwe natuur'. Bij natuurontwikkeling gaat het om een menselijk, doelbewust streven naar bepaalde natuurwaarden. Dat is bijvoorbeeld omvormen van landbouwgrond naar natuur, zoals gebeurd is bij het eiland Tiengemeten. De communicatie over deze 'nieuwe natuur' in Nederland is onderwerp van dit proefschrift en in het kort komt het er op neer dat gezocht wordt naar dieptelagen in deze communicatie. Of meer specifieker: in dit proefschrift wordt onderzocht of (impliciete) religieuze elementen in de communicatie over natuur een rol spelen.

Hoofdstuk 2 laat zien dat er naast een beleidsmatige verandering ten aanzien van het Nederlandse natuurbeleid, ook sprake is van een relationele verandering. Deze 'contextual turn' houdt in dat de overheid in toenemende mate, al dan niet vrijwillig, in onderhandeling treedt met burgers en belangengroepen. Deze veranderende context heeft logischerwijs invloed op de overheidscommunicatie. Haar karakter is de achterliggende decennia ingrijpend veranderd: van een sterke nadruk op informatieoverdracht naar een situatie waarbij communicatie en beleid min of meer samenvloeien. Hoofdstuk 2 concludeert dat beleid in toenemende mate is het creëren van een juiste setting waar discursieve uitwisseling kan plaatsvinden. Verschillende perspectieven en vooronderstellingen spelen hierbij een rol. Deze dienen aan het licht te komen en verhelderd te worden. Een debat wordt in hoofdstuk 2 daarom beschouwt als een georganiseerde dialoog tussen concurrerende normatieve posities. In wezen draait het in een debat om de interpretatie van observaties op basis van het eigen wereldbeeld. Dit impliceert voor communicatieprofessionals bij de overheid dat ze oog hebben voor de normatieve posities in het debat en dat ze in staat zijn deze te articuleren en te interpreteren.

Om uit te vinden wat de uiteindelijke bestemming, de 'telos' van de overheidscommunicatie is, wordt in hoofdstuk 3 het model van normatieve praktijken, zo- als ontwikkeld binnen de Reformatorische Wijsbegeerte geïntroduceerd. Hoofdstuk 3 betoogt dat overheidscommunicatie primair draait om het toekennen van betekenis aan het handelen van de overheid. Daarom wordt overheidscommunicatie opgevat als talige interactie, met als bestemming het delen van betekenissen. Als dit op een overtuigende en oprechte manier gebeurt, mag je veronderstellen dat sociale legitimatie volgt. Overtuigend en oprecht communiceren wil zeggen dat de communicatie van de overheid consistent is met haar 'telos', in overeenstemming is het hoe de overheid op dit moment handelt en het consistent is met hoe eerder is gecommuniceerd. Het is wel van belang het aspect van sociale legitimatie te onderscheiden van de vraag of alle stakeholders er inhoudelijk mee eens zijn. Als de communicatie deugt mag je veronderstellen dat de stakeholders de inbreng van de overheid als een terechte inbreng ervaren en een eventueel besluit aanvaarden, ook als men het er niet mee eens is. Democratie betekent niet zo zeer dat burgers het eens zijn met het actuele beleid en handelen van de overheid, maar dat zij vertrouwen kunnen hebben in de voorafgaande democratische besluitvorming.

Hoofdstuk 4 beschrijft de verschillende manieren waarop in het Nederlandse natuurbeleid tegen natuur wordt aangekeken. Een belangrijk markeringspunt is de jaren 80/90 van de vorige eeuw. Sinds die tijd is er een herleving zichtbaar van met name het wildernis natuurbeeld. Werd in het verleden wildernis in de natuu herkend, sinds de jaren 80/90 wordt meer en meer natuur naar ons beeld van wildernis 'gemaakt'. In feite is hier sprake van een paradox: natuurontwikkeling verbindt zich met huidige ideeën over technologie, terwijl het streeft naar een prehistorisch type natuur dat onafhankelijk is van de mens. In Nederland is er in zekere zin dan ook sprake van een technische benadering van natuur.

In hoofdstuk 4 wordt ook geconcludeerd dat in de natuur betekenisvolle ervaringen worden opgegaan. Een scala aan benamingen is te vinden in de literatuur hierover, bijvoorbeeld: sublieme ervaringen, ervaringen van het numineuze, spirituele/religieuze ervaringen, epifanie, etc. In zijn algemeenheid zou je kunnen spreken over transcendente ervaringen: ervaringen die mensen brengen tot voorbij dat wat men rationeel kan bevatten en waarbij ze zich aangesproken voelen door 'het Andere', wat dat ook precies moge zijn, maar wat ervaren of beschouwd wordt als het heilig. Dit soort ervaringen kunnen ertoe leidden dat er in de communicatie over natuur elementen zijn terug te vinden met een religieuze connotatie, zogenoemde 'religious subtexts'. Uit dit proefschrift blijkt dat deze 'religious subtexts' niet noodzakelijkerwijs theologisch van aard zijn. De voorbeelden in hoofdstuk 4 maken eerder duidelijk dat er veelal geen sprake is van een persoonlijke God of andere bovennatuurlijke verwijzing. 
Hoofdstuk 5 vergelijkt op basis van een kwalitatieve casestudie over Tiengemeten de ervaringen van bezoekers met het discourse van de beleids- en communicatie professionals, de zogenoemde sleutelfiguren. Voor Tiengemeten is gekozen omdat dit natuurontwikkelingsproject exemplarisch is voor het actuele denken over natuur in Nederland. Onder de respondenten een bepaalde terughoudendheid is waar te nemen bij het gebruik van het begrip 'echte' natuur. 'Echte' natuur heeft bij de respondenten vaak de connotatie van spontane en autonome ontwikkeling, minder die van puur of origineel. Hoofdstuk 5 concludeert dat onder de respondenten het wildernis natuurbeeld dominant in combinatie met wat een light versie van het Arcadisch natuurbeeld wordt genoemd. Hierbij is geen aantoonbaar verschil tussen bezoekers en de sleutelfiguren. Je zou kunnen zegen dat er onder de respondenten sprake lijkt te zijn van een hybridisatie van wildernis en Arcadisch, van ruig en rustig, van appreciatie van nieuwe natuur in combinatie met melancholische trekjes richting het verleden.

Hoofdstuk 5 een signaleert een discrepantie tussen wat de sleutelfiguren aan de ene kant communiceren en wat bezoekers van Tiengemeten aan de andere kant ervaren. Sleutelfiguren geven aan dat de publieke communicatie het gevoel zou moeten oproepen 'daar wil ik zijn, dat is wat ik wil ervaren'. Maar de casestudy over Tiengemeten laat zien dat woorden en beelden die in het discourse gebruikt worden, niet altijd overeenkomen met de ervaringen van bezoekers als ze eenmaal op de beschreven plaats zijn. De ervaringen die de communicatiemiddelen suggereren, komen niet altijd overeen met de ervaringen ter plekke. Met andere woorden, het ervaren van een plaats is van invloed op hoe de communicatie hierover wordt geëvalueerd. Daarom wordt in hoofdstuk 5 gepleit voor het voortdurend afstemmen van de publieke communicatie op de actuele stand van de natuur ontwikkeling. Geadviseerd wordt om 'grote woorden' of te waarden-geladen communicatie te vermijden en in de communicatie aan te sluiten bij wat natuurontwikkelingsprojecten daadwerkelijk brengen. Dit is zowel belangrijk vanuit het oogpunt van vertrouwen, als om scepsis bij bezoekers te voorkomen en niet het risico te lopen dat de publieke communicatie contraproductief wordt.

Hoofdstuk 6 beschrijft eveneens op basis van een kwalitatieve casestudy over Tiengemeten in hoeverre er in de communicatie van bezoekers van Tiengemeten dieptelagen zichtbaar zijn. Wat opvalt is dat woorden en beelden die bezoekers gebruiken om hun belevingen te omschrijven vooral passend zijn bij het wildernis natuurbeeld. Kijkend naar welke ervaringen in verwoorde belevingen te identificeren zijn, valt op dat er allereerst ervaringen zijn die sterk verbonden zijn met de specifieke context van Tiengemeten. Gevoelens van verlies van de (agrarische) identiteit en ervaringen die te maken hebben met het gegeven dat Tiengemeten een eiland is. De overtocht met de pont is voor veel respondenten cruciaal. Het geeft gevoelens van 'de ene wereld los laten, de andere wereld betreden.' Een van de respondenten sprak hierover ook in termen van een andere dimensie van het bestaan. Maar er waren ook ervaringen die uitdrukking geven aan wat genoemd is de appellerende werking van natuur. Dit betreft gevoelens van jezelf opladen, jezelf (her)vinden, etc. in de natuur, als ook ervaringen die samenhangen met het ervaren van de grootsheid, het overweldigende van de natuur. Deze laatste categorie van ervaringen is meer passend bij het wildernis natuurbeeld, terwijl met name de ervaring van verlies van de (agrarische) identiteit meer passend is bij een Arcadische natuurbeeld.

In hoofdstuk 7 worden de belangrijkste resultaten van dit proefschrift bediscussieerd. Dit wordt gegaan op basis van vier thema's. In het eerste thema normatieve karakter van communicatie wordt betoogd dat het de rol van communicatieprofessionals is om normatieve elementen in het discours te signaleren, te articuleren en te interpreteren. Oftewel, het expliciteren van al dan niet niet-congruente frames en de daaraan verbonden wereldbeelden in het discours inzichtelijk maken. Inclusief dat van de overheid zelf. De overheid is in haar communicatie-uitingen zoals onpartijdig als mogelijk. Maar de communicatie over natuur laat zien dat bij deze neutraliteit vragen te stellen zijn. Hoewel niet expliciet, maar via de communicatie van Ngo's, die in de context van dit proefschrift functioneren als gedelegeerde uitvoerders van het Nederlandse natuurbeleid, worden bepaalde beelden, i.c.

frames over natuur gecommuniceerd. Met andere woorden, het valt op dat in de communicatie van de Ngo's sprake is van 'religious subtexts': er lijkt vooral een appèl gedaan te worden op 'een vlucht uit het dagelijks leven', de natuur wordt voorgesteld als de 'betere wereld' en in de communicatie wordt een soort belofte gedaan - in de natuur kan je jezelf weer (her)vinden.

In het tweede thema 'religious subtexts' in de communicatie wordt de vraag opgeroepen in hoeverre de overheid vanuit haar vermeende neutraliteit moet sturen op de communicatie van Ngo's. Behoren 'religieuze subtexts' wel tot de communicatie over uitwerkingen van het overheidsbeleid? Omdat in het geval van dit proefschrift Ngo's de facto functioneren als gedelegeerde uitvoerders van het Nederlandse natuurbeleid, wijst dit tweede thema op een mogelijke spanning. Als Ngo's (expliciet) bepaalde visies op de natuur - met behulp van 'religieuze subtexts' - communiceren, wordt de suggestie gewekt dat de overheid deze 'subtexts' ondersteunt. Voor een Ngo is het passend een bepaalde visie naar voren te brengen om steun te werven voor haar acties. Maar in dit tweede thema wordt 
geconcludeerd dat het niet tot de taak van de overheid behoort om een visie op de natuur en natuurbeleid te promoten dat (impliciete) religieuze elementen bevat. Derhalve onderstreept dit tweede thema de noodzaak om na te denken over de relatie tussen de overheid en Ngo's met betrekking tot communicatie, dat wil zeggen bewustwording van het onderscheid en de noodzaak van onderlinge afstemming in geval van nauwe samenwerking.

In het derde thema paradox in natuurervaringen worden de uitkomsten van dit proefschrift in een breder cultureel kader ten aanzien van natuurontwikkeling geplaatst. In dit thema wordt een paradox van beheersing en beleving gesignaleerd: natuur maken 'naar ons beeld van de natuur' en ook wildernis-natuur, liefst zonder te veel menselijke invloeden, willen beleven. Deze paradox lijkt een culturele onderlegger van veel nieuwe natuurontwikkelingsprojecten te zijn. Met andere woorden, natuurontwikkeling wordt niet langer alleen gedreven door ecologische belangen. In het hedendaagse 'wildernisverlangen' manifesteert zich ook een bepaalde vorm van antropocentrisch denken, omdat het de natuurbeleving van mensen centraal stelt. Maar omdat (nieuwe) natuurprojecten ook plaatsen zijn om zinervaringen op te doen, wordt in dit thema geconcludeerd dat (nieuwe) natuurprojecten, zoals bijvoorbeeld Tiengemeten, naast ecologisch ook maatschappelijk waardevol zijn.

In vierde thema natuur ervaringen en seculiere spiritualiteit wordt betoogt dat we in een seculiere samenleving de mediërende rol van inrichting en onderhoud van natuurparken niet uit het oog moeten verliezen. Het aanleggen van (nieuwe) natuurgebieden is niet een technisch-neutrale bezigheid. Het is niet 'slechts' natuur, het is niet enkel een 'openbare ruimte'. Door natuurgebieden op een bepaalde manier in te richten of te onderhouden worden er als het ware condities geschapen voor bepaalde betekenisvolle ervaringen. Met andere woorden: betekenisvolle ervaringen in de natuur zijn gemedieerd door inrichting en onderhoud. Wellicht is religiositeit niet langer intrinsiek verbonden met een specifieke gemeenschap, maar waar het natuurervaringen betreft is het individuele van de ervaring betrekkelijk: er is altijd een overheid, een natuurorganisatie, etc. die 'voorwaarden' (door beleid, inrichting en beheer) schept of heeft geschapen. Met onze vormgevende visie en communicatie kunnen we de 'benefits' van de natuur tot ons laten komen. Paradoxaal genoeg zullen we de natuur in zekere zin moeten 'bezitten' om genoemde kwaliteiten van de natuur te ontsluiten. Dit proefschrift laat met deze conclusie een ander licht schijnen op het concept van natuurontwikkeling en indirect op het Nederlandse natuurbeleid.
Tenslotte, in dit proefschrift is op basis van vijf studies, weergeven als afzonderlijke hoofdstuk $2 \mathrm{t} / \mathrm{m} 6$, zowel theoretisch als empirisch materiaal verzameld om een antwoord te geven op de vraag of in de communicatie over natuur in Nederland (impliciete) religieuze elementen een rol spelen. De conclusie is dat dit proefschrift laat zien dat religieuze elementen in de communicatie over natuur een rol spelen. Deze blijken verband te hebben met betekenisvolle ervaringen die mensen in de natuur kunnen opdoen. In deze betekenisvolle ervaringen is een religieuze dieptedimensie te ontdekken. Deze religieuze dieptedimensie is de reden dat er in de communicatie over natuur sprake is van 'religious subtexts'. Het is van belang het woord 'subtext' te benadrukken, want de communicatie over de natuur is tot op zekere hoogte 'religionised' maar er is geen sprake van een vermelding van een persoonlijke God of andere bovennatuurlijke verwijzing. Dit proefschrift laat ook zien dat de religieuze dieptedimensie bij bezoekers nog niet zo expliciet aan de oppervlakte komt in wat zij zeggen. Dat maakt dat dit proefschrift, naast de vraag naar gepastheid van 'religious subtexts' in de communicatie over natuur, ook betwijfelt of deze 'subtexts' overtuigen vanuit het perspectief van de bezoekers. 


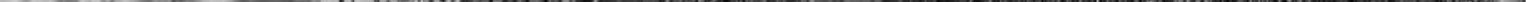

\section{Curriculum Vitae}

Peter Jansen (1977) was born in Amersfoort. After preparatory secondary vocational education (Lagere Land- en Tuinbouw School) and senior secondary vocational education (Middelbare Land- en Tuinbouwschool), he completed a propaedeutic year in Garden and Landscape Architecture at Van Hall Larenstein University of Applied Sciences in Velp. He subsequently obtained a professional Bachelor Degree in Garden \& Landscape Management at HAS University of Applied Sciences in ‘s-Hertogenbosch.

In 2001 after receiving his Bachelor Degree, he began working as a teacher at Van Lodensteincollege, a secondary school in Kesteren and Amersfoort. Beside his work as a teacher, he studied at Wageningen University. In 2006, he obtained his Master Degree with distinction (cum laude) for which he wrote two theses: one thesis in Education and Competence Studies (Onderwijskunde, supervisors: Prof. Dr E. Schuurman and Dr H.J.M. Biemans) and one in Forest and Nature Conservation Policy (Bos- en Natuurbeleid, supervisors: Dr B.H.M. Elands en Dr A.E. Buijs). In 2005, he emigrated with his family to Curaçao, an island in the Caribbean. After several years working for a youth development organization, he decided to return to the Netherlands. Since the summer of 2008, he has been working as a senior lecturer and researcher at the department of Journalism and Communication Studies at Ede Christian University of Applied Sciences.

Since 2002, he has been married to Jacomijn van Dijke (1980); together, they have two daughters, Nelissa (2005) and Corinda (2008). He became a member of the City Counsel of Ede after the elections of 2014 
The research described in this thesis was financially supported by the Doctoral Grant for Teachers (in Dutch: Promotiebeurs voor Leraren, project number 023.001.002) and by the Incentive Fund Open Access, both from the Netherlands Organisation for Scientific Research (NWO). Financial support is also received from the Scholten Codes Fund and the Pro Rege Study Fund.

Financial support from Wageningen University for printing of this thesis is gratefully acknowledged.

\section{Printed by}

VanderPerk Groep

Graphic Design

TinekeWerkt.nl

Photography

Reinier van Dijke 


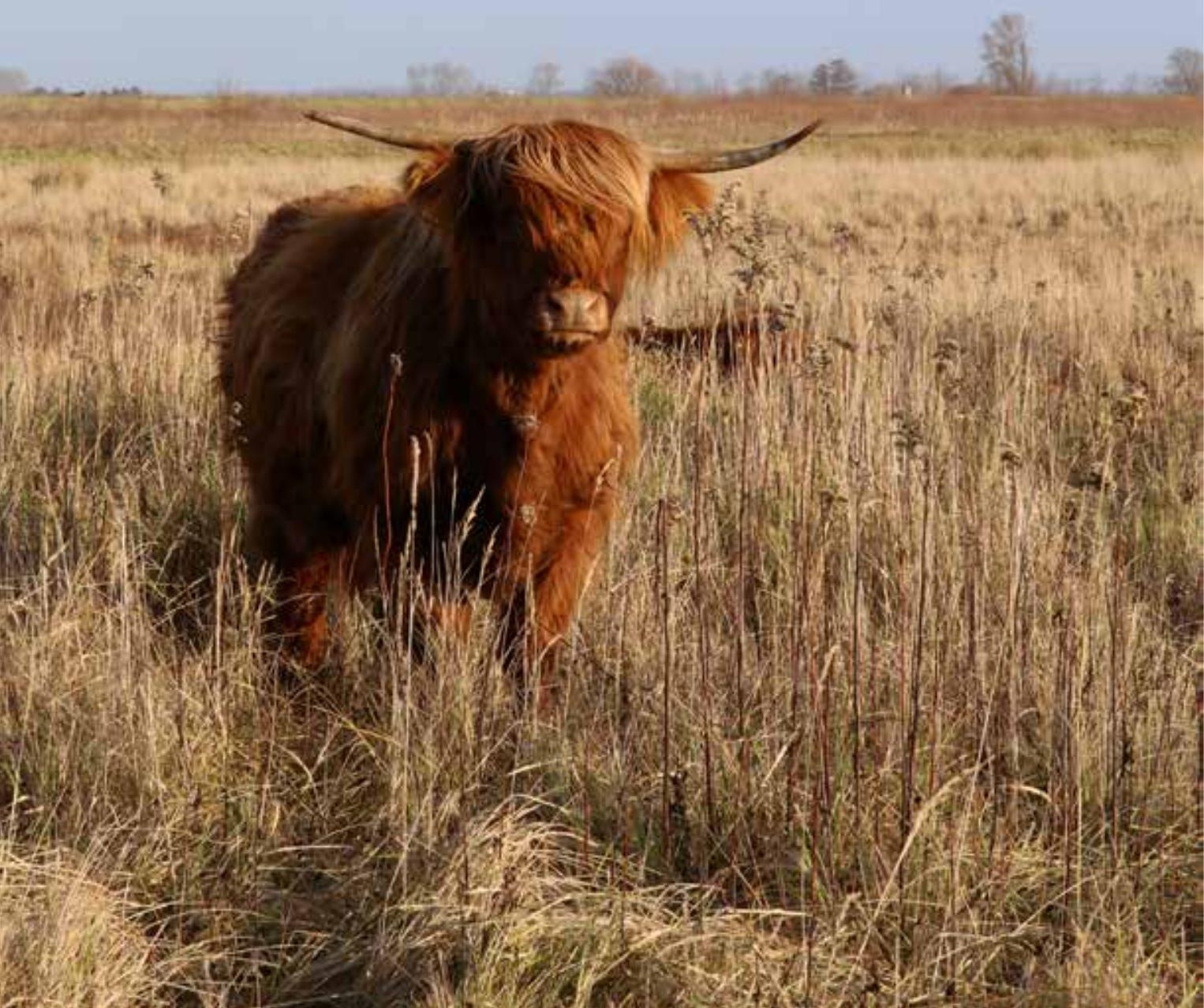

